\title{
AMBIENT NOISE AND SURFACE WAVE DISSIPATION IN THE OCEAN
}

\author{
by \\ Francis C. Felizardo \\ B.S. in Civil Engineering, magna cum laude, 1985 \\ University of the Philippines \\ S.M. Civil Engineering, 1990 \\ Massachusetts Institute of Technology \\ SUBMITTED IN PARTIAL FULFILLMENT OF THE \\ REQUIREMENTS FOR THE DEGREE OF \\ DOCTOR OF PHILOSOPHY \\ CIVIL \& OCEANOGRAPHIC ENGINEERING
}

\begin{tabular}{c} 
MARINE \\
BIOLOGICAL \\
LAEORATORY \\
\hline LIBRARY' \\
\hline $\begin{array}{c}\text { WOODS HOLE, MASS. } \\
\text { W. H. O. I. }\end{array}$ \\
\hline
\end{tabular}

at the

\section{MASSACHUSETTS INSTITUTE OF TECHNOLOGY}

and the

WOODS HOLE OCEANOGRAPHIC INSTITUTION

June 1993

(C) Massachusetts Institute of Technology and

Woods Hole Oceanographic Institution, 1993

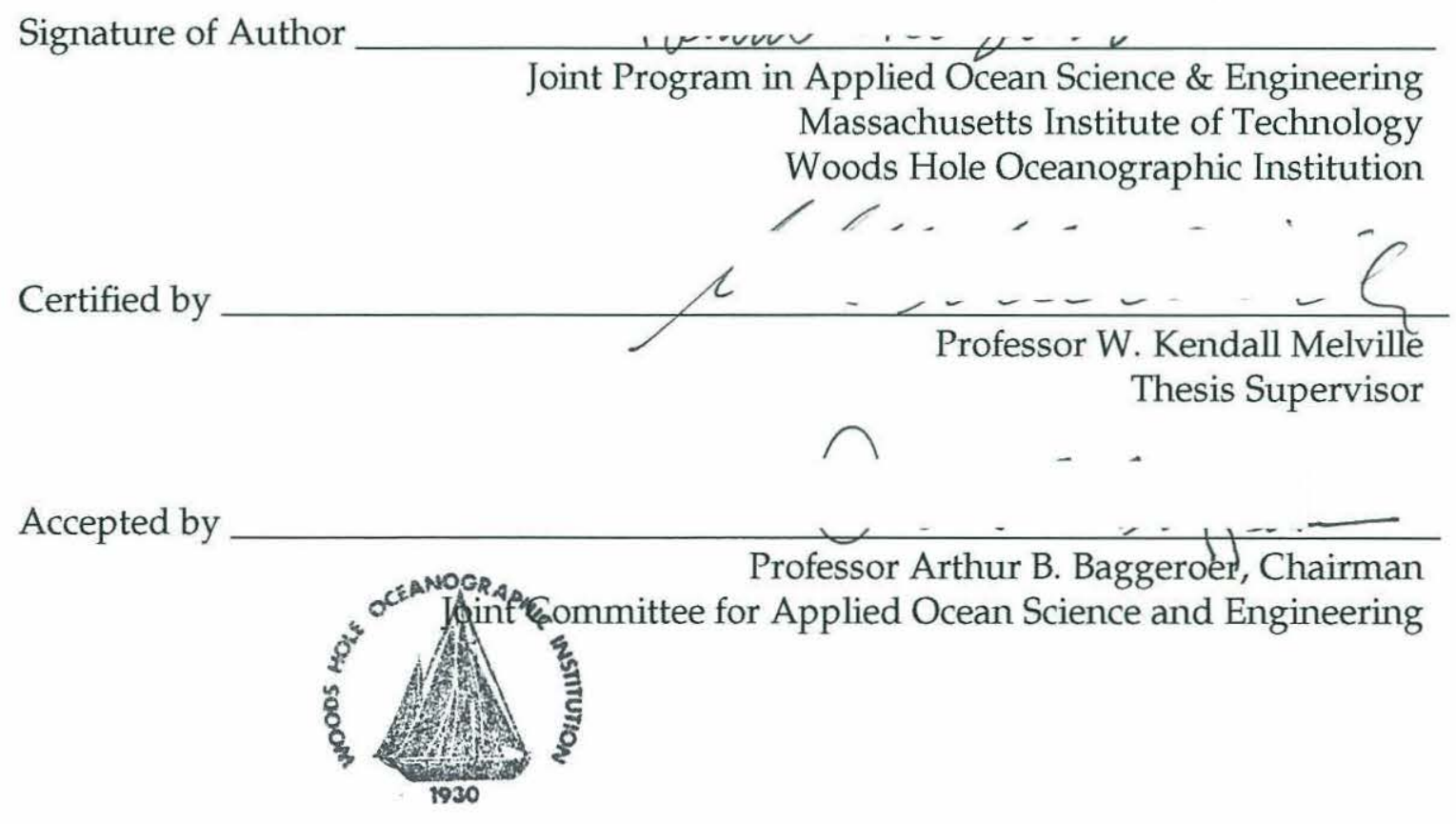




\title{
AMBIENT NOISE AND SURFACE WAVE DISSIPATION IN THE OCEAN
}

\author{
by \\ Francis C. Felizardo \\ Submitted to the Massachusetts Institute of Technology and \\ the Woods Hole Oceanographic Institution on June 21, 1993 \\ partial fulfillment of the requirements for the degree of \\ Doctor in Philosophy in Civil and Oceanographic Engineering
}

\begin{abstract}
There is a growing consensus that the sound generated by breaking waves is responsible for much of the ambient noise level in the ocean. While numerous field measurements have shown a strong correlation between the ambient noise spectrum level $(\mathrm{N})$ in the range $100 \mathrm{~Hz}$ to $25 \mathrm{kHz}$ and wind speed in the ocean, very little has been done to establish a comparable correlation between the ambient noise spectrum level and surface wave field parameters. The difficulty in establishing this relationship is remarkable given that the frequency and intensity of wave breaking are dependent on the characteristics of the wave field.

In Fall 1991, an experiment was conducted from the research platform Flip 130 kilometers off the coast of Oregon, where the ambient noise between 2.5 and $25 \mathrm{kHz}$, the wind speed, and the sea surface elevation using wire wave gauges were measured.

The correlation between $\mathrm{N}$ and the root mean square wave amplitude $a$ was found to be poor but could be improved if the swell was filtered out from the wave elevation time series. The influence of swell on the value of $a$ was disproportionate to the level of ambient noise since its characteristics were not directly due to the local wind-wave conditions. Observations of the dependence of the high frequency wind waves and the directional wave spectrum under turning winds suggested that the high frequency wave components responded more quickly to changes in the wind speed and wind direction than the energy-containing frequencies.

The ambient noise level also correlated well with the root mean square wave slope $s$. This is consistent with previous laboratory measurements which showed that the steepness of a packet of waves correlates with the strength of wave breaking and with characteristics of breaking waves such as loss of momentum flux, dissipation, initial volume of air entrained, mixing, and sound generation.

Comparisons of surface wave dissipation estimates using field measurements and models developed by Phillips (1985) and Hasselmann (1974) show that although the two models have very different forms, they give values that are comparable in magnitude. The relationship between the ambient noise level and log of dissipation give correlation coefficients $(0.93-0.95)$ that are comparable to those between ambient noise and wind speed. The mean square acoustic pressure was shown to vary with the dissipation, with $\mathrm{p}^{2} \propto \mathrm{D}^{0.6-0.8}$. The results suggest that measurements of ambient sound may prove to be useful in inferring surface wave dissipation.
\end{abstract}

Thesis Supervisor: W. Kendall Melville

Title: Visiting Professor, Department of Civil \& Environmental Engineering 


\section{ACKNOWLEDGMENTS}

In one of my favorite Star Trek episodes, Captain Jean-Luc Picard realizes that his life is like a tapestry where if he pulls a thread, however seemingly insignificant, the whole fabric falls apart. I mentioned this because I feel that the tapestry of family, friends and colleagues whose assistance, support and guidance have brought me where I am now deserves to be recognized and acknowledged. While I can't thank everyone who really deserves my gratitude for helping me complete this work, I would like to take this opportunity to thank as many people as I can.

I would like to thank Ken Melville for his guidance, supervision, and for providing support and a great learning environment. I have grown under his guidance in so many ways. I would also like to thank the members of my research committee, Ira Dyer, Ole Madsen and Tim Stanton for their advice, suggestions and helpful comments to this work.

This research would never have been completed were it not for the excellent, excellent people who were at the Noise on Basalts and Sediments (NOBS) Experiment. Anatol Rozenberg helped me design, build and set-up the directional hydrophone. The capacitance wire wave gauge was built by Feng Chi Wang for an earlier experiment. He helped me check it and showed me how to use it. He came over evenings after work and weekends just for that.

John Hildebrand of SIO was the principal investigator during NOBS, Vince Pavlicek was the chief engineer, Tony Aja and Pam Scott were the technicians, Sean Wiggins and Rob Sohn were John's grad students. Some of the data from environmental instruments were provided by Jerry Smith and Rob Pinkel of SIO. I worked closely with Greg Bullard in helping set up their wave gauges and in extracting the NOBS data from their archives. Greg also lent me his surfboard and wetsuit on my first trip to San Diego.

Thanks also go to the crew of the RP Flip, DeWitt Efird (master), Tom Golfinos, Doug Richardson and George Swann. Tony Parra's excellent cooking made the isolation during NOBS much easier to bear.

I could never have made it through MIT without Pat Dixon. I owe her a big debt of gratitude for all the big and little things that she has done for us and for me personally. I heard she's moved on to much bigger things at MIT and I can only feel glad that more people can avail of the help that she can offer. Vicky Murphy, Ken's contract administrator at MIT has helped me fix a few of botched up POs.

A year ago, Ken, Anatol and I moved to Scripps and started up our lab here. Like all start-ups, we had a lot of administrative screw ups. The people at the MPL and PORD administrative staff, Louis Herrera, Mary Sisti, Joanie Sumption, Gail Smith, Michelle Frybager, Ruth Bachmann and Diane Wells cheerfully and patiently helped us mend our ways. 
I would also like to thank the new members of Ken's 'garage company' here at SIO: Eric Terrill, Derek Quigley, Andrew Keyes, Alexey Federov, Robert Shear and Thuc Luu. Alexey helped check and critique some of my math. Thuc helped scan some figures and prepare text.

This section is not complete unless I mention some of the many people who helped me relax and take time off from my academic life. Jeptha and Emily Wade were my hosts during my first few years in the US. Frank and Connie Nolido and their family also served as my informal hosts during the same period. I value their friendship immensely. I also enjoyed my friendships with the members of the MIT Filipino Students Association. MIT was a much more welcoming and enjoyable place because of them. I will miss the many friends I made at the Internet Filipino culture newsgroup (soc.culture.filipino). I will miss the interesting discussions and debates I got myself into.

I spent several vacations with my pinsans here in the US - the Riveras in St. Louis, the Estradas in Chicago, and the Estradas in Los Angeles who to me are like my kid sisters. More than anyone else, they have made this place a home away from home. I would like to thank my parents, Reynaldo and Amosa, and my brothers and sisters (Kuya, Ate May, Dindo, Leo, Tess, Paul and Bob) for the support that they have given me. My wife's family - Mommy and Daddy, Ate Jingle, Andy and Dixie, and my best enemy Twinkle are now family to me also.

You know I won't get this far without mentioning Ken Melville's group at MIT with whom I have shared a lot of times with and who are arguably one of the closest friends I've ever had: Mark Loewen, Andy Jessup, Gunnar Tomasson, Eric Lamarre, Feng Chi Wang, Anatol Rozenberg, Analia Barrantes, Eng-Soon Chan, Diane Ronan, Anne Slinn, Ge Wei, and Dong Zhou. Nalin Wikramanayake (I think I finally learned to pronounce that correctly) was the first non-Filipino I met in the US so I guess he deserves a mention too. Actually, we're really good friends. I will miss all the good times we've had.

And just as you thought this would never end, we're here. To my wife Leah, who has known me for ten years and counting since last February. I don't know how she just puts up with me. I thank her for her love, her understanding and her support. I really, really admire and love her. To her, I dedicate this work. 


\section{TABLE OF CONTENTS}

TITLE

ABSTRACT

ACKNOWLEDGEMENTS ……………………….......................................

TABLE OF CONTENTS $\quad$.................................................................................

LIST OF SYMBOLS $\quad$..............................................................................

LIST OF FIGURES $\quad$.................................................................................. 11

LIST OF TABLES

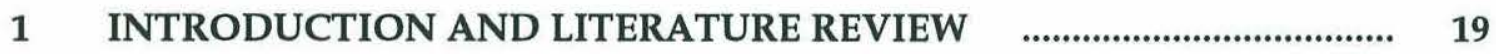

1.1 Ambient noise and wind speed. ………………................................ 21

1.2 Ambient noise and the wave field. ………………………………..... $\quad 30$

1.3 Sound generated by breaking waves. ………………………............. 34

1.4 Breaking wave sound and dissipation. $\quad$................................................ 41

1.5 Wind wave dissipation. $\quad$....................................................................... 44

1.6 Breaking wave dissipation models. ………….................................... 47

1.7 Thesis outline. ………………………………………………..... 55

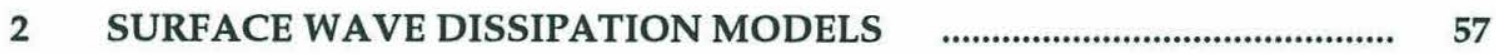

$2.1 \quad$ Equilibrium spectral slopes. $\quad$.............................................................. 57

2.2 Phillips dissipation model. $\quad$.................................................................... $\quad 59$

$2.3 \quad$ Hasselmann dissipation model. $\quad$.......................................................... 62

2.4 Reduced Hasselmann dissipation estimate. $\quad$.......................................... 63

$3 \quad$ EXPERIMENT ……............................................................................ 66

3.1 NOBS description. …………………………………………....... 66 


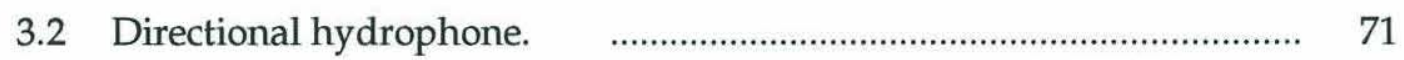

3.2.1 Data sampling and preprocessing. ………………………...... $\quad 75$

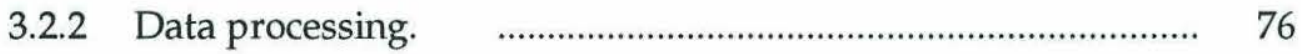

3.3 Environmental instruments. …………………………………....... 80

3.4 Wave gauges, accelerometers and compass. ……………………...... 82

3.5 Wave spectra. ……………………………………………….... 86

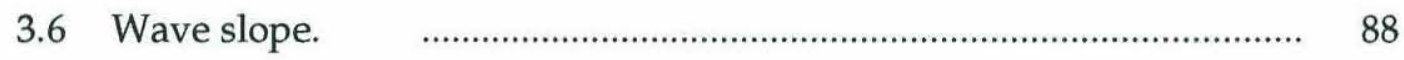

3.7 Directional wave height spectra. …………….................................. 92

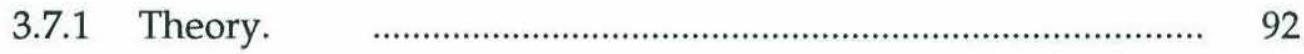

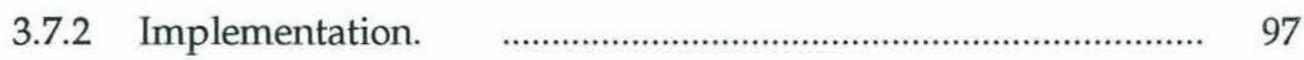

4 OBSERVATIONS …….................................................................... 99

4.1 General conditions. …………………………................................. 99

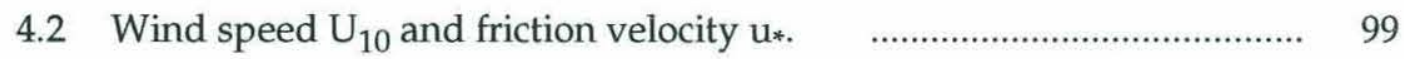

4.3 Wave spectrum characteristics. ……………………………….......... 102

4.4 Ambient noise.

4.5 Directional wave spectra. $\quad$............................................................... 110

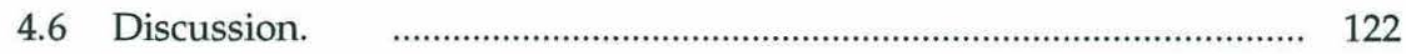

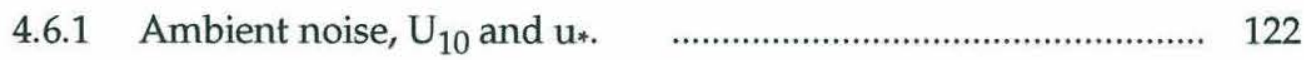

4.6.2 Ambient noise and wave parameters. ………….................... 128

4.6.3 Ambient noise and wind-wave development. ....................... 140

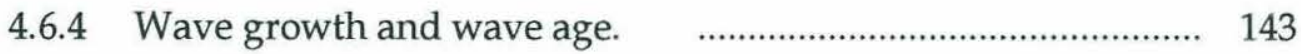

4.6.5 Wave spectrum characteristics. ……………........................ 147

$5 \quad$ SPECTRAL DISSIPATION ESTIMATES ….......................................... 152

5.1 Experimental results. …………………………………………....... 153

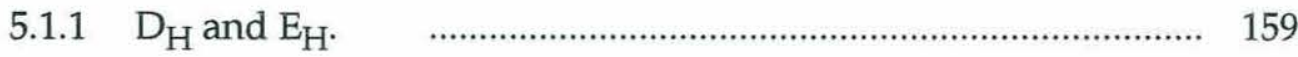




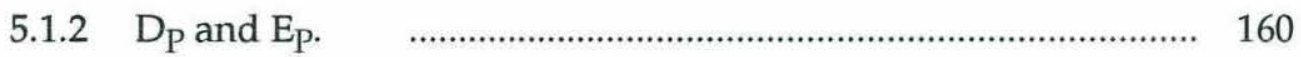

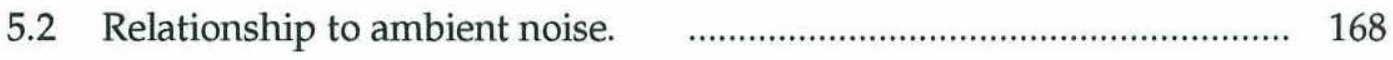

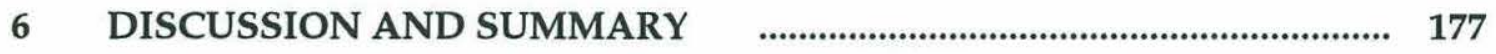

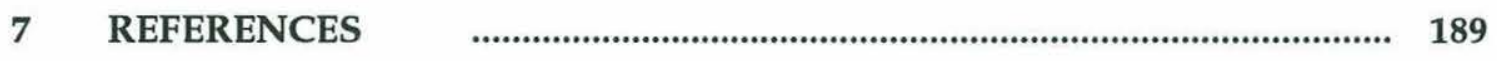

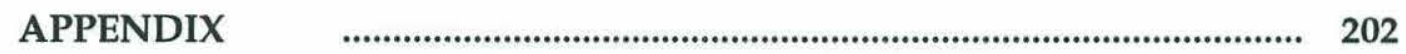

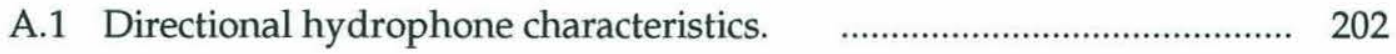

A.1.1 The hydrophone equation. ….............................................. 202

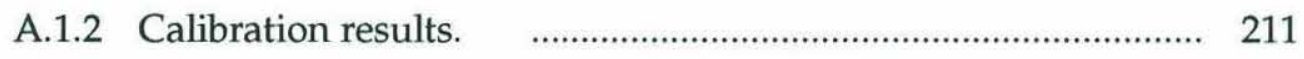

A.1.3 Array gain computations. …......................................... 211 


\section{LIST OF SYMBOLS}

\begin{tabular}{|c|c|}
\hline A & Fourier transform of $a(t)$ \\
\hline a & sea surface elevation \\
\hline$a_{c}$ & calibrated sea surface elevation \\
\hline$a_{j}$ & sea surface elevation measured by sensor i \\
\hline$a^{\prime}$ & root mean square surface wave amplitude \\
\hline$a_{w}$ & wind wave root mean square amplitude \\
\hline $\mathrm{B}$ & hydrophone beam pattern \\
\hline$C_{D}$ & drag coefficient \\
\hline $\mathrm{C}_{\mathrm{ij}}$ & cross spectral density between $\mathrm{i}$ and $\mathrm{j}$ \\
\hline$\hat{\mathrm{C}}_{\mathrm{ij}}$ & cross spectral density estimate between $\mathrm{i}$ and $\mathrm{j}$ \\
\hline$C_{x y}$ & correlation coefficient between $\mathrm{x}$ and $\mathrm{y}$ \\
\hline $\mathrm{c}_{\mathrm{b}}$ & phase speed of the breaking wave crest \\
\hline$c_{p}$ & phase speed of the peak frequency of the wave spectrum \\
\hline$c_{g}$ & group velocity vector \\
\hline$c_{H}$ & Komen et al (1984) dissipation coefficient $\left[3.33 \times 10^{-5}\right]$ \\
\hline$c_{p}, c_{v}$ & specific heats \\
\hline $\mathrm{D}$ & surface wave energy dissipation per unit area \\
\hline$D$ & fraction of surface wave energy dissipated by a breaking wave. \\
\hline $\mathrm{D}_{\mathrm{H}}$ & D computed using the Komen et al (1984) formula \\
\hline $\mathrm{D}_{\mathrm{P}}$ & D computed using the Phillips (1985) formula \\
\hline $\mathrm{D}_{\mathrm{T}}$ & D computed using the Thorpe (1992) formula \\
\hline $\mathrm{d}$ & surface wave spectral dissipation per unit area \\
\hline $\mathrm{d}_{\mathrm{s}}$ & energy dissipated/unit length by a steady breaking wave \\
\hline $\mathrm{d}_{\mathrm{u}}$ & energy dissipated/unit length by an unsteady breaking wave \\
\hline $\mathrm{E}_{\mathrm{H}}$ & reduced $\mathrm{D}_{\mathrm{H}}$ computed using integral wave parameters \\
\hline $\mathrm{E}_{\mathrm{P}}$ & reduced $D_{P}$ computed using wind and wave parameters \\
\hline $\mathrm{e}$ & sea surface variance \\
\hline$\tilde{\mathrm{e}}$ & non-dimensional sea surface variance \\
\hline f & frequency \\
\hline $\bar{f}$ & mean frequency of the surface wave spectrum \\
\hline$f$ & void fraction of air in water \\
\hline$f_{d}$ & resonant frequency of a dipole source \\
\hline $\mathrm{f}_{\mathrm{o}}$ & resonant frequency of an oscillating bubble \\
\hline $\mathrm{g}$ & gravitational acceleration $[9.81 \mathrm{~m} / \mathrm{s}]$ \\
\hline $\mathrm{H}_{\mathrm{s}}$ & significant wave height \\
\hline I & surface wave directional spreading function \\
\hline$I$ & acoustic intensity \\
\hline $\mathrm{j}$ & imaginary number $=\sqrt{-1}$ \\
\hline $\mathrm{k}$ & wavenumber, $\mathrm{k}=$ magnitude $(\mathbf{k})$ \\
\hline $\mathbf{k}$ & wavenumber vector, $\mathbf{k}=\left(\mathrm{k}_{\mathrm{x}}, \mathrm{k}_{\mathrm{y}}\right)$ \\
\hline $\mathrm{L}$ & dipole moment \\
\hline $\mathrm{N}$ & ambient noise spectrum level [dB re $1 \mu \mathrm{Pa}^{2} / 1 \mathrm{~Hz}$ ] \\
\hline $\mathrm{P}$ & ambient pressure \\
\hline $\mathrm{p}^{2}$ & acoustic mean square pressure \\
\hline $\mathrm{p}^{2}$ ref & reference pressure [usually $1 \mu \mathrm{Pa}^{2}$ ] \\
\hline
\end{tabular}




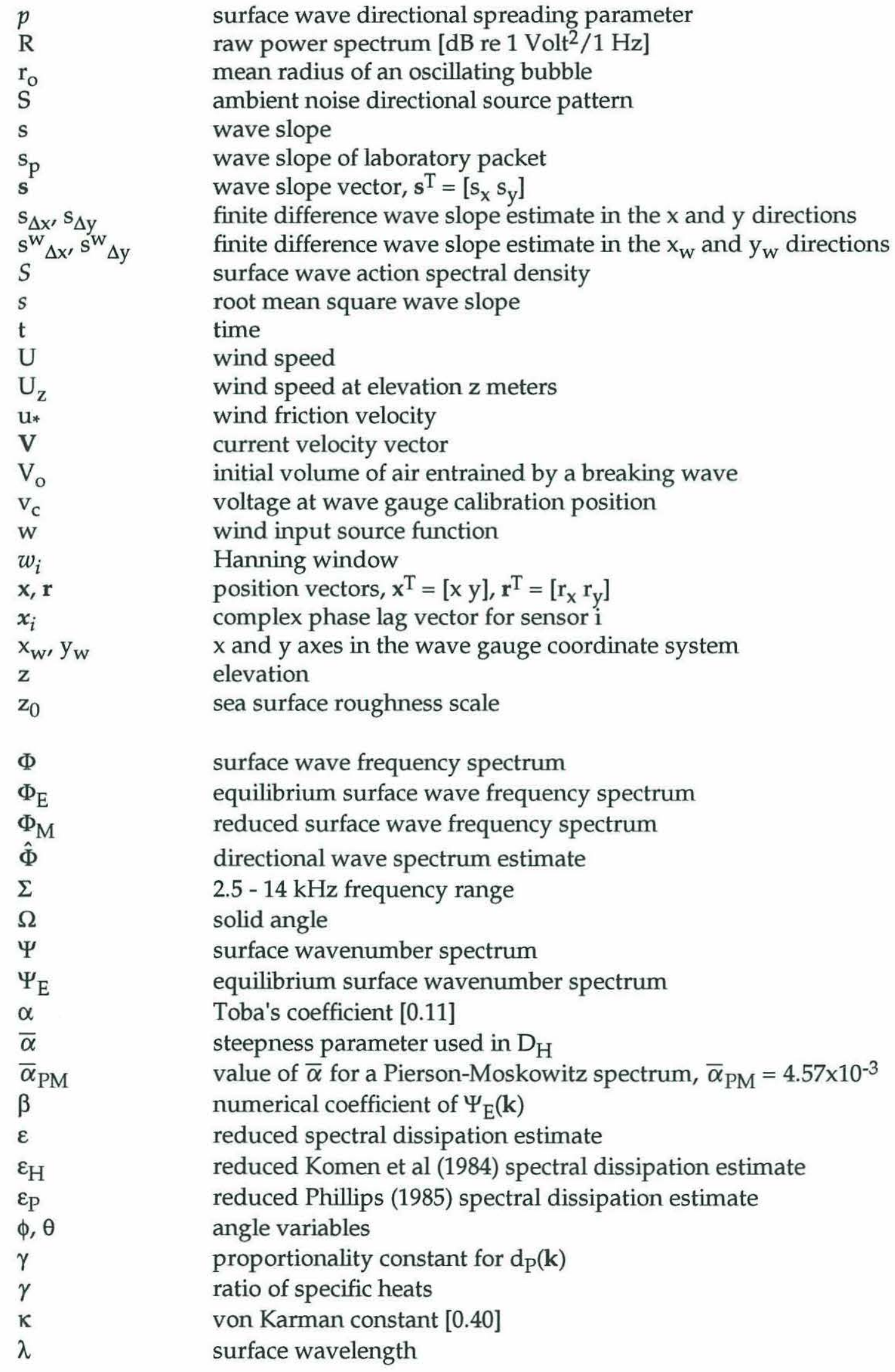




$\begin{array}{ll}\lambda_{\mathrm{p}} & \text { wavelength of the surface wave peak frequency } \\ \theta_{\mathrm{d}} & \text { computed surface wave direction } \\ \theta_{\mathrm{F}} & \text { Flip azimuth } \\ \theta_{\text {wind }} & \text { azimuth of wind direction (direction wind is headed towards) } \\ \rho & \text { density } \\ \rho_{\mathrm{w}} & \text { sea water density density }\left[1025 \mathrm{~kg} / \mathrm{m}^{3}\right] \\ \rho_{\mathrm{a}} & \text { air density density }\left[1.3 \mathrm{~kg} / \mathrm{m}^{3}\right] \\ \sigma^{2} & \text { variance } \\ \omega & \text { radian frequency } \omega=2 \pi \mathrm{f} \\ \omega_{\mathrm{p}} & \text { peak radian frequency of the surface wave spectrum } \\ \omega & \text { mean radian frequency of the surface wave spectrum } \\ \xi & \text { fraction of breaking crests } \\ \text { AG } & \text { hydrophone array gain } \\ \text { SA } & \text { signal amplification } \\ \text { SL } & \text { hydrophone sensitivity level }\end{array}$




\section{LIST OF FIGURES}

Figure 1.1. Sources and spectrum levels of deep water ambient noise (Wenz, 1962).

Figure 1.2. A plot comparing the wind speed measured by a vectoraveraging wind recorder (VAWR) type anemometer 3.5 meters above the sea surface and by a WOTAN at $4.3 \mathrm{kHz}$ ambient noise frequency (Evans et al, 1984).

Figure 1.3. Plot of $\log U$ versus the ambient noise level at 8 and $14.5 \mathrm{kHz}$. Note that when U exceeds 10 meters/second, the departure from the linear relationship between $\mathrm{N}$ and $\log \mathrm{U}$ becomes significant (Farmer \& Lemon, 1984).

Figure 1.4. Ambient noise spectrum level during a breaking event detected by a hydrophone 14 meters below sea level. The background spectrum immediately before breaking shown in the first plot is reproduced by the dashed lines in the second and third plots. The $90 \%$ confidence level is indicated by the vertical bars in each plot (Farmer \& Vagle, 1989).

Figure 1.5. The sound radiated by a typical oscillating bubble generated by a laboratory spilling breaker can be described as an exponentially damped sinusoid (Medwin \& Beaky, 1989).

Figure 1.6. Plot showing the observed peak frequency $\mathrm{f}_{\mathrm{ob}}$ of the pressure spectrum from breaking waves and the predicted resonant frequency $\mathrm{f}_{\mathrm{R}}$ for a cylindrical bubble cloud computed using the method described by Lu et al (1990) [ Loewen \& Melville, 1992].

Figure 1.7. A plot showing that the acoustic energy radiated by a breaking event $\mathrm{E}_{\mathrm{a}}$ is proportional to the dissipated mechanical wave energy $\mathrm{E}_{\mathrm{L}}$. $\mathrm{E}_{\mathrm{a}}$ was scaled by the square of the product of the center wavenumber of the surface wave packet $k_{c}$ and the depth of the wave tank h (Loewen \& Melville, 1991a).

Figure 1.8. The energy dissipated by a breaking event $D$ is proportional to the initial volume per unit width of air entrained by a breaking wave $V_{0}$. The figure shows laboratory results from three different wave packets of various wave slope (Loewen, 1991).

Figure 1.9. A plot of the wind induced wave growth $W / \Psi(\mathbf{k})$ as a function of frequency at $\mathrm{u}_{*}=0.45 \mathrm{~m} / \mathrm{s}$. Data were compiled from seven different investigations by Plant (1982). Dashed lines show limits of Equation 1.14. Data from Snyder et al (1981) is indicated by shaded area (Plant, 1982). 
Figure 1.10. Log-log plot of the fraction of breaking waves $\xi$ versus the inverse wave age $\left(\mathrm{U}_{10} / \mathrm{c}_{\mathrm{p}}\right)$. Data was taken from various field investigations: Longuet-Higgins \& Smith (1983)- $\mathbf{0}$, Weissman et al (1984) WA+K, Thorpe \& Humphries (1980)- O, Katsaros \& Atakturk (1991) - •, and Thorpe (1990) - $\square$, (Thorpe, 1992).

Figure 1.11. Normalized speed of a breaking event $\left(c_{b} / c_{p}\right)$ versus the phase speed $c_{p}$ (Ding, 1993).

Figure 3.1. A map showing the location of Flip during the NOBS experiment $\left(43^{\circ} 42^{\prime} \mathrm{N}, 125^{\circ} 59^{\prime} \mathrm{W}\right)$. The site is approximately $130 \mathrm{~km}$ west of Oregon and $600 \mathrm{~km}$ south of Vancouver Island.

Figure 3.2. A schematic diagram of Flip showing the locations of the different instruments used in the experiment. The capacitance wire wave gauge is 15 meters and the resistance wire wave gauge is 17 meters from the hull of Flip.

Figure 3.3. Schematic diagram of the sampling and preprocessing method used in gathering and storing data.

Figure 3.4. A photograph of the directional hydrophone taken immediately after the platform 'flipped' from vertical to horizontal attitude. The photograph was taken from a location near the bow of Flip. The hydrophone is the dark circular disk at the center of the photograph.

Figure 3.4. A close-up photograph of the directional hydrophone taken while Flip was in port in San Diego. The instruments in the foreground are the SIO Doppler sonars used to image the sea surface.

Figure 3.6. A simplified diagram of the directional hydrophone and the mounting. The dimensions are not to scale.

Figure 3.7. Sensitivity level (SL) of the directional hydrophone in $\mathrm{dB}$ re $1 \mathrm{Volt}^{2} / 1 \mu \mathrm{Pa}^{2}$ obtained from a calibration of the instrument at the Naval Ocean Systems Center.

Figure 3.8 Plot of array gain (AG) of the directional hydrophone as a function of frequency. AG values computed from NOSC calibration are denoted by $\mathrm{O}$.

Figure 3.9. Some typical ambient noise power spectra in $\mathrm{dB}$ re $1 \mu \mathrm{Pa}^{2} /$ $1 \mathrm{~Hz}$ at various wind speeds.

Figure 3.10. Photograph of the 4-wire resistance wave gauge array 
and the capacitance wire wave gauge (heavy black cable) deployed at the end of the boom. The heavy white cable near the center of the photograph is the ground wire.

Figure 3.11. Photograph of the bottom spreader bar being lowered to position prior to full deployment of the wire wave gauge array. The bar is made of stainless steel and each arm is 0.5 meters long.

Figure 3.12. Plot showing the values for the four different field calibrations of the capacitance wire wave gauge. The dashed line is a linear least square fit of the sample points. The equation for the best linear least squares fit is

$$
\mathrm{a}_{\mathrm{c}}(\mathrm{m})=0.541 \mathrm{v}_{\mathrm{c}}(\text { Volts })+0.800
$$

where $a_{c}$ is the calibration position and $v_{c}$ is the mean voltage reading at that position.

Figure 3.13. Plots showing the characteristics of a typical wave height power spectrum. The data were sampled at $8 \mathrm{~Hz}$. The spectra were computed from a one hour wave gauge record with $U_{10}=8 \mathrm{~m} / \mathrm{s}$.

a) Power spectrum computed from 1024-point FFTs used throughout this work. b) Power spectrum over the same period computed from 2560-point FFTs to resolve the lower frequency spectral peaks.

Figure 3.14. Plan view schematic diagram showing the location and orientation of the resistance wave gauge array and the coordinate systems used in computing the wave slope $s$.

Figure 3.15. Plan view of schematic diagram showing the coordinate system used in computing the directional wave spectrum $\Phi(\omega, \theta)$.

Figure 4.1. Time series of $\mathrm{U}_{10}, \mathrm{u}_{*}$ and the wind direction during the NOBS experiment. Note that the wind rapidly changes direction on Julian day 278 after blowing from the North for approximately six days.

Figure 4.2. Some typical 512-point wave height spectra $\Phi(\mathrm{f})\left[\mathrm{m}^{2} / \mathrm{Hz}\right]$ taken from one hour records at the start of each day of the NOBS experiment. a) 269. b) 270. c) 271. d) 272. e) 273. f) 274. g) 275. h) 276 . i) 277. j) 278. k) 279. 1) 280 .

Figure 4.3. a) Root mean square amplitude $a(\mathrm{~m})$ and significant wave height $\mathrm{H}_{\mathrm{s}}(\mathrm{m})$ computed from the full spectrum. b) Mean frequency computed from the full spectrum $\overline{\mathrm{f}}$. c) $a_{w}$ and $\mathrm{H}_{\mathrm{s}}$ 
computed with swell filtered out. d) Mean frequency with the swell filtered out $\overline{\mathrm{f}}_{\mathrm{w}}$.

Figure 4.4. Time series of $\log \Phi\left(\mathrm{m}^{2} / \mathrm{Hz}\right)$ at selected frequencies:

Figure 4.5. Time series of the RMS wave slope $s$ (Equation 3.12) computed from the resistance wire wave gauge array data.

Figure 4.6. Time series of the ambient noise spectrum level $\mathrm{N}$ ( $\mathrm{dB}$ re $1 \mu \mathrm{Pa}^{2} / 1 \mathrm{~Hz}$ ) at selected WOTAN frequencies. Note the similarities between these time series and the $\mathrm{U}_{10}$ time series. In particular, note the drop and in $\mathrm{N}$ on Julian day 278 . a) $4.3 \mathrm{kHz}$. b) $8.0 \mathrm{kHz}$.

c) $14.0 \mathrm{kHz} . \mathrm{d}$ ) wide band [2-14 $\mathrm{kHz}$ or $\Sigma]$.

Figure 4.7. Directional wave spectra from 0.25 to $1 \mathrm{~Hz}$. The sequence of plots demonstrate the evolution of the wave field in response to turning winds on Julian day 278. The $x$-axis for the plots is frequency in $\mathrm{Hz}$ and the $\mathrm{y}$-axis is wave direction $\theta_{\mathrm{d}}$. Dashed lines indicate the direction the wind is blowing towards $\left(\theta_{\text {wind }}\right)$.

Figure 4.8. The directional wave spectra in Figure 4.7 normalized by the spectrum level at the given frequency to highlight the directional distribution of the wave energy in response to turning winds on Julian day 278 . The $x$-axis for the plots is frequency in Hertz and the $y$-axis is the wave direction $\theta_{\mathrm{d}}$. Dashed lines indicate the direction the wind is blowing towards $\left(\theta_{\text {wind }}\right)$.

Figure 4.9. Plot of $U_{10}$ vs. selected $N$ frequencies. Lines indicate regression lines obtained by various investigations. This study - solid line, Evans et al (1984) - short dash, Lemon et al (1984) - dash-dot-dot, Vagle et al (1990) - dash-dot.

Figure 4.10. Plot of $u_{*}$ vs. selected $N$ frequencies. The figure shows a good correlation between $\mathrm{u}^{*}$ and $\mathrm{N}$.

Figure 4.11. a) Plot of the drag coefficient $C_{D}=\left(u_{*} / U_{10}\right)^{2}$ as a function of $\left(U_{10} / c_{p}\right)$. b) Plot relating the inverse wave age based on $U_{10}$ and on $\mathrm{u}_{*}$.

Figure 4.12. Plot of $\mathrm{N}$ vs. the root mean square wave amplitude $a$. The scatter in the data give correlation coefficients $=0.67$ for all the plots.

Figure 4.13. Plot of $\mathrm{N}$ vs. $a_{w}$. The data was high pass filtered to eliminate the contribution of swell energy to $a_{w}$. The figure shows a significant 
improvement in the correlation between ambient noise $\mathrm{N}$ and the RMS wind wave amplitude $a_{w}$ (see Table 4.3).

Figure 4.14. The high frequency surface wave energy and ambient 133 noise $\mathrm{N}$ are well-correlated. a) Plot of $\mathrm{N}$ vs. $\Phi(0.5 \mathrm{~Hz})$. b) Plot of $\mathrm{N}$ vs. $\Phi(0.75 \mathrm{~Hz})$. c) Plot of N vs. $\Phi(1.0 \mathrm{~Hz})$.

Figure 4.15. Plot of wave slope versus the ambient noise $\mathrm{N}$ at selected frequencies.

Figure 4.16. Plot of wave slope $s$ as a function of wind speed $U_{10}$, wind stress $\mathrm{u}_{*}$ and inverse wave age $\left(\mathrm{U}_{10} / \mathrm{c}_{\mathrm{p}}\right)$ and $\left(\mathrm{u}_{*} / \mathrm{c}_{\mathrm{p}}\right)$.

Figure 4.17. The wind and wave time series during NOBS can be subdivided into steady and unsteady conditions. The data in section II were considered steady while those in sections I, III and IV were considered unsteady.

Figure 4.18. Plot of $\mathrm{N}(4.3 \mathrm{kHz})$ versus selected wind and wave parameters. The plots on the left are for data during unsteady conditions. The plots on the right are for data during steady conditions.

Figure 4.19. Plot of $U_{10}$ versus selected wave parameters. The plots on the left are for data during unsteady conditions. The plots on the right are for data during steady conditions.

Figure 4.20. Plot of the non-dimensional wave energy versus the inverse wave age.

Figure 4.21. Selected normalized 1-hour wave spectra. The spectra were taken from wave height records sampled at 0000 UT. The solid line denotes $\Phi$ for $\alpha=0.11$ (Equation 4.17). a) Spectra from 12 day record. b) Spectra from fully developed seas.

Figure 5.1. Comparisons of the time series of the log of the total dissipation $\left[\mathrm{kg} / \mathrm{s}^{3}\right]$ computed using the formulas given in Table 5.1. a) $D_{H}$ (solid line) and $E_{H}$ (short dash), b) $D_{P}$ (solid line) and $E_{P}$ (short dash). c) $\mathrm{D}_{\mathrm{P}}$ (solid line) and $\mathrm{D}_{\mathrm{H}}$ (short dash), b) $\mathrm{E}_{\mathrm{P}}$ (solid line) and $\mathrm{E}_{\mathrm{H}}$ - (short dash).

Figure 5.2. Plots showing the spectral dependence of the $\mathrm{KHH}$ dissipation $\mathrm{d}_{\mathrm{H}}(\mathrm{f})$ and $\varepsilon_{\mathrm{H}}(\mathrm{f})\left[\mathrm{kg} / \mathrm{s}^{3} \mathrm{~Hz}\right]$. These spectral estimates were computed from the wave spectra shown in Figure 2.4. Numbers below the plots indicate the Julian day from which the plots 
are taken.

Figure 5.3. Plot showing a good correlation between $0.89 a_{w}^{2} \bar{\omega}_{w}^{3}$ and $\alpha \mathrm{u} * \mathrm{~g}$.

The regression line has a slope $=1$ and the correlation coefficient between the two parameters is 0.92 .

Figure 5.4. a) Plots showing the time series of the $\Phi_{\mathrm{M}}$ and $\Phi_{\mathrm{E}}$ coefficients $0.89 a_{w}^{2} \bar{\omega}_{w}^{3}$ (solid line), $\alpha \mathrm{u}_{*} \mathrm{~g}$ (short dash) $\left[\mathrm{m}^{2} / \mathrm{s}^{3}\right]$. b) Plot showing the relative magnitude of the difference between $0.89 a_{w}^{2} \bar{\omega}_{w}^{3}$ and $\alpha u * g$.

Figure 5.5. Plots showing the spectral dependence of the Phillips dissipation $d_{P}(f)$ and $\varepsilon_{P}(f)\left[\mathrm{kg} / \mathrm{s}^{3} \mathrm{~Hz}\right]$. These spectral estimates were computed from the wave spectra shown in Figure 2.4. Numbers below the plots indicate the Julian day from which the plots were taken.

Figure 5.6. Plots of the $\mathrm{KHH}$ dissipation $\mathrm{D}_{\mathrm{H}}$ versus ambient noise at selected frequencies. The dashed line indicates the regression computed from the data (see Table 5.1 for the coefficients).

Figure 5.7. Plots of the reduced $\mathrm{KHH}$ dissipation $\mathrm{E}_{\mathrm{H}}$ versus ambient noise at selected frequencies. The dashed line indicates the regression computed from the data (see Table 5.1 for the coefficients).

Figure 5.8. Plots of the Phillips (1985) dissipation $D_{P}$ versus ambient noise at selected frequencies. The dashed line indicates the regression computed from the data (see Table 5.1. for the coefficients).

Figure 5.9. Plots of the Philips (1985) dissipation $\mathrm{E}_{\mathrm{P}}$ versus ambient noise at selected frequencies. The dashed line indicates the regression computed from the data (see Table 5.2 for the coefficients).

Figure A.1. Plot of the Sensitivity Level (SL).

Figure A.2. a) Plot of the beam pattern of the hydrophone at $1 \mathrm{kHz}$. b) Plot of the beam pattern of the hydrophone at $3 \mathrm{kHz}$. c) Plot of the beam pattern of the hydrophone at $4 \mathrm{kHz}$. d) Plot of the beam pattern of the hydrophone at $5 \mathrm{kHz}$. e) Plot of the beam pattern of the hydrophone at $10 \mathrm{kHz}$. f) Plot of the beam pattern of the hydrophone at $15 \mathrm{kHz} . \mathrm{g}$ ) Plot of the beam pattern of the hydrophone at $20 \mathrm{kHz}$.

Figure A.3. Geometry of Urick's (1986) model for the distribution of sound sources at the ocean surface. 
Figure A.4. Urick's (1986) cos $\phi$ model of the vertical distribution of ambient noise (dashed line) agrees well with measurements of Axelrod et al (1965) at $891 \mathrm{~Hz}$ (Urick, 1986).

Figure A.5. Geometry for the coordinate transformation from the true coordinate system to the hydrophone coordinate system.

Figure A.6. Plot showing the array gain (AG) of the directional hydrophone computed from the available beam patterns. Dipole source assumed - 0 , omnidirectional source assumed - $\bullet$. The line indicates the fit to the data used in computing $\mathrm{N}(\mathrm{f})$ [Equation 3.1]. 


\section{LIST OF TABLES}

Table 1.1. Approximate relationship between sea state and wind speed [adapted from Wenz (1962)].

Table 1.2. Some coefficients for the WOTAN expression and the equivalent power laws obtained from previous field measurements.

Table 1.3. Some typical wind speeds predicted by the WOTAN equation and coefficients given in Table 1.2.

Table 1.4. Correlation coefficients between $N, H_{s}$ and $U$ (Penhallow \& Dietz, 1964). 31

Table 3.1. Instruments used in this experiment.

Table 4.1. Some coefficients for the WOTAN expression and the equivalent power laws computed from NOBS data (c.f. Table 1.2 and Figure 4.9).

Table 4.2. Linear regression coefficients relating $\mathrm{u} *$ and $\mathrm{N}$ and the equivalent power laws computed from NOBS data (c.f. Figure 4.10).

Table 4.3. Linear regression coefficients relating $a_{w}$ and $N$ and the equivalent power laws computed from NOBS data.

Table 4.4. Power law coefficients for the ambient sound pressure level and wave slope s, and the corresponding correlation coefficients $C_{x y}$ between $\mathrm{N}$ and $\log s$.

Table 4.5. Linear correlation coefficients between $\mathrm{N}(4.3 \mathrm{kHz})$, and the log of several wind and wave parameters.

Table 4.6. Coefficients of the non-dimensional wind and wave growth expression.

Table 5.1. Dissipation estimates (see Chapter 2 for full details.)

Table 5.2. Coefficients for the ambient noise and dissipation expression and the equivalent power laws computed from NOBS data.

Table A.1. Numerical values of AG and SL used in Equation 3.1. 
Chapter 1

\section{INTRODUCTION AND LITERATURE REVIEW}

Since World War II, there has been considerable research interest in understanding the natural sources of underwater sound. The initial motivation of the acoustic community was primarily the desire to characterize the nature of these noise sources so underwater instruments could be used more effectively. Underwater ambient noise was primarily of military and biological interest. As our knowledge of the characteristics of underwater sound grew, oceanographers and acousticians began to realize that ambient noise could be used to understand the physical processes that are responsible for generating underwater sound and ultimately, increasing our knowledge of the atmosphere and the ocean.

In numerous experiments in the last decade, investigators have used passive acoustic measurements of underwater ambient noise to infer the wind speed [e.g., Shaw, Watts \& Rossby (1978) and Evans, Watts, Halpern \& Bourassa (1984)], estimate the intensity and distribution of breaking waves (Farmer and Vagle, 1988), track the spatial and temporal position of breaking waves in the vicinity of the hydrophones [e.g., Ding \& Farmer (1992), Crowther \& Hansla (1993)], infer the existence of an acoustic wave guide formed by the relatively high bubble concentration near the ocean surface (Farmer \& Vagle, 1989). Laboratory experiments on breaking waves by Melville, Loewen, Felizardo, Jessup \& Buckingham (1988) and by Loewen \& Melville (1991a) suggest that the dissipation of surface wave energy in the ocean may also be inferred from measurements of ambient noise. This hypothesis is further explored in this work.

It is now believed that the sound generated by bubble mechanisms in breaking waves is the primary source of the wind-dependent ambient noise in the ocean [Kerman 
$(1988,1992)]$. Yet while decades of field measurements have shown a well-correlated power law relationship between underwater ambient noise and wind speed [Knudsen, Alford \& Emling (1948), Urick (1986)], very few measurements have tried to establish a similar correlation between ambient noise and wave field parameters. The few measurements that have attempted to do so show that ambient noise does not correlate well with the significant wave height [Penhallow \& Dietz (1964), Perrone (1969), Farmer \& Lemon (1984)]. This is remarkable given that breaking waves are more directly related to the characteristics of the wave field rather than the wind. We believe that this could be due to the fact that swell and the longer wave components which do not break contribute a significant proportion to the variance of the wave field. The laboratory measurements of Melville and his coworkers [e.g., Melville et al (1988), Loewen \& Melville (1991a), Lamarre \& Melville (1991)] suggest that both the sound generation and the dissipation in breaking waves are coupled by the air entrainment process and that this is why laboratory experiments have shown that these two parameters correlate with each other.

The aim of this thesis is to investigate whether comparable correlations hold in the ocean. To this end, we have conducted an experiment measuring ambient noise, wind, and the surface wave field from a floating platform (RP Flip) off the coast of Oregon. These measurements will be used to investigate the correlation between ambient sound and a number of surface wave parameters, especially surface wave energy dissipation which is most directly related to wave breaking. In addition, we will use these measurements to obtain empirical relationships between these parameters.

Before proceeding to a discussion of the field experiment and the data, we will, in this chapter, review the available literature on ambient noise measurements and their 
correlation with wind and wave parameters. We will then discuss the mechanisms for sound generation associated with breaking waves. Later, we will examine the results of some recent laboratory experiments on breaking waves. Finally, we will discuss the literature on surface wave energy dissipation.

\subsection{Ambient noise and the wind speed.}

Pioneering underwater sound measurements by Knudsen, Alford \& Emling (1948) during World War II showed that the noise spectrum level N(f) in the $100 \mathrm{~Hz}-25 \mathrm{kHz}$ range correlates with sea state. The study resulted in what is now commonly referred to as the 'Knudsen curves', a family of curves showing a slope of approximately -17 to $-20 \mathrm{~dB}$ per decade within this frequency range as a function of wind speed. The Knudsen curves have been verified by other investigators over the last few decades [see Wenz (1962) and Urick (1986) for a review]. Figure 1.1 reproduces a figure from Wenz (1962) which summarizes the sources and sound spectrum levels of the prevailing ambient noise in the ocean. The Knudsen curves in this figure are expressed in terms of sea state which was commonly used at the time. Table 1.1 gives the equivalent wind speed for a given sea state. Although this figure is three decades old, it is still useful in providing a general idea of the sound levels and sources in the ocean.

Functional expressions between ambient noise and wind speed were first proposed by Crouch \& Burt (1972) using field measurements by Piggott (1964) and Perrone (1969). They found that this relationship can be described by the empirical relation 


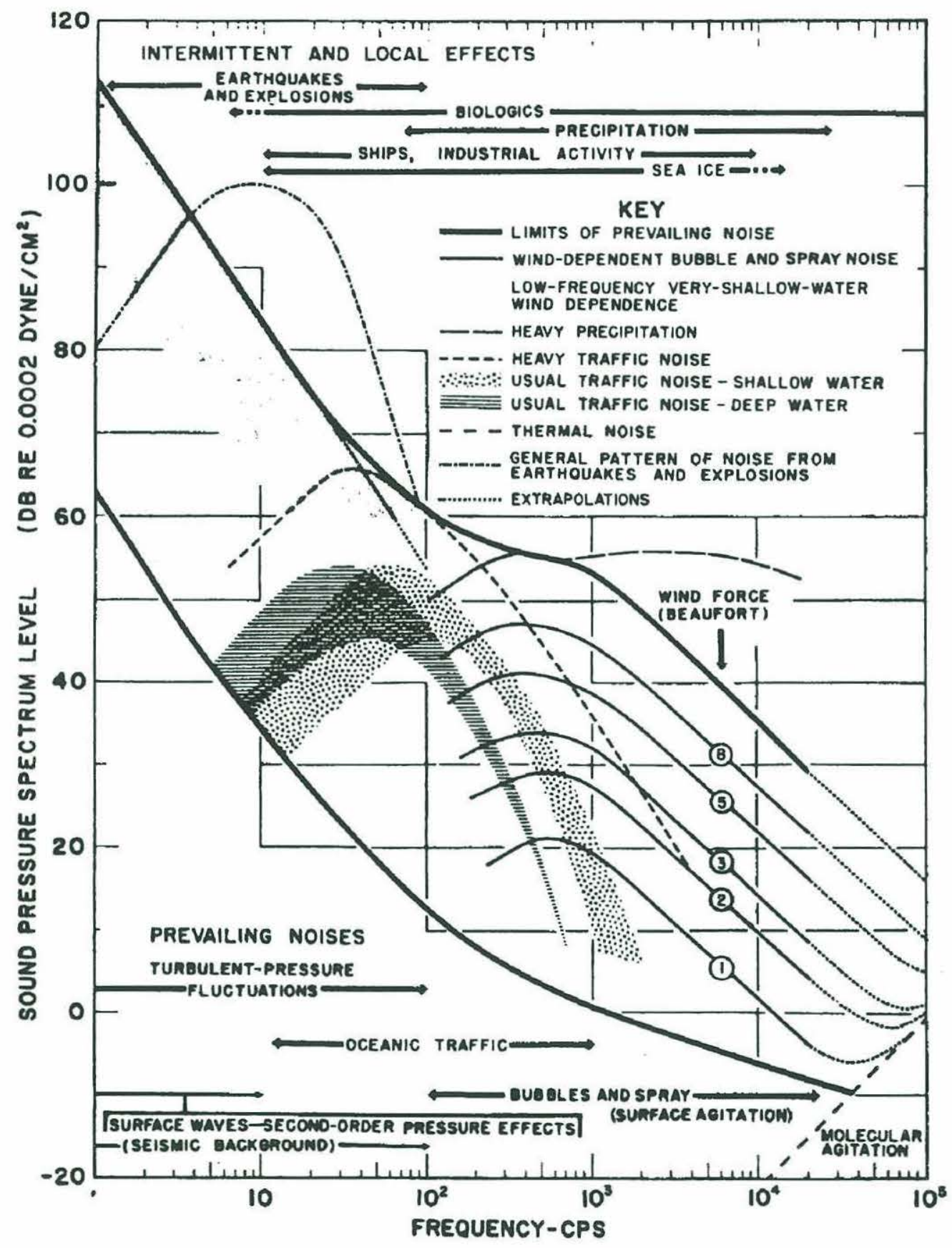

Figure 1.1. Sources and spectrum level of deep water ambient noise (Wenz, 1962). 
Table 1.1. Approximate relationship between sea state and wind speed [adapted from Wenz (1962)].

\begin{tabular}{|c|c|c|}
\hline $\begin{array}{c}\text { Beaufort } \\
\text { sea state }\end{array}$ & $\begin{array}{c}\text { Mean wind speed } \\
(\mathrm{m} / \mathrm{s})\end{array}$ & Sea criteria \\
\hline 0 & $<0.5$ & Mirror-like \\
\hline 1 & 1.0 & Ripples \\
\hline 2 & 2.5 & Small wavelets \\
\hline 3 & 4.5 & Large wavelets, scattered whitecaps \\
\hline 4 & 7.0 & Small waves, frequent whitecaps \\
\hline 5 & 10.0 & Large waves, whitecaps everywhere, spray \\
\hline 6 & 12.5 & Heaped-up sea, blown spray, streaks \\
\hline 7 & 15.5 & Moderately high long waves, spindrift \\
\hline 8 & 19.0 & \\
\hline
\end{tabular}

$$
N(f)=N_{1}(f)+20 n(f) \log U
$$

where $f$ is the frequency at which the measurements were made, $N_{1}(f)$ is the noise spectrum level at 1-knot wind speed, $U$ is the wind speed in knots, and $n(f)$ is a coefficient of order one. $\mathrm{N}$ is the wind dependent noise spectrum level ( $\mathrm{dB}$ re 1 $\left.\operatorname{mbar}^{2} / 1 \mathrm{~Hz}\right)$

$$
N(f)=10 \log \left[\frac{p^{2}(f)}{p_{\text {ref }}^{2}}\right]
$$

where $\mathrm{p}^{2}(\mathrm{f})$ is the ambient noise power spectrum. Crouch \& Burt (1972) used a reference pressure $\mathrm{p}_{\mathrm{ref}}^{2}=1 \mathrm{mbar}^{2}$ which is different from the current System International (SI) unit $\mathrm{p}_{\text {ref }}^{2}=1 \mu \mathrm{Pa}^{2}$ more commonly used today. Note that Equation

\footnotetext{
${ }^{1}$ All common logarithms used in this work are denoted by 'log' while natural logarithms are denoted by 'ln'.
} 


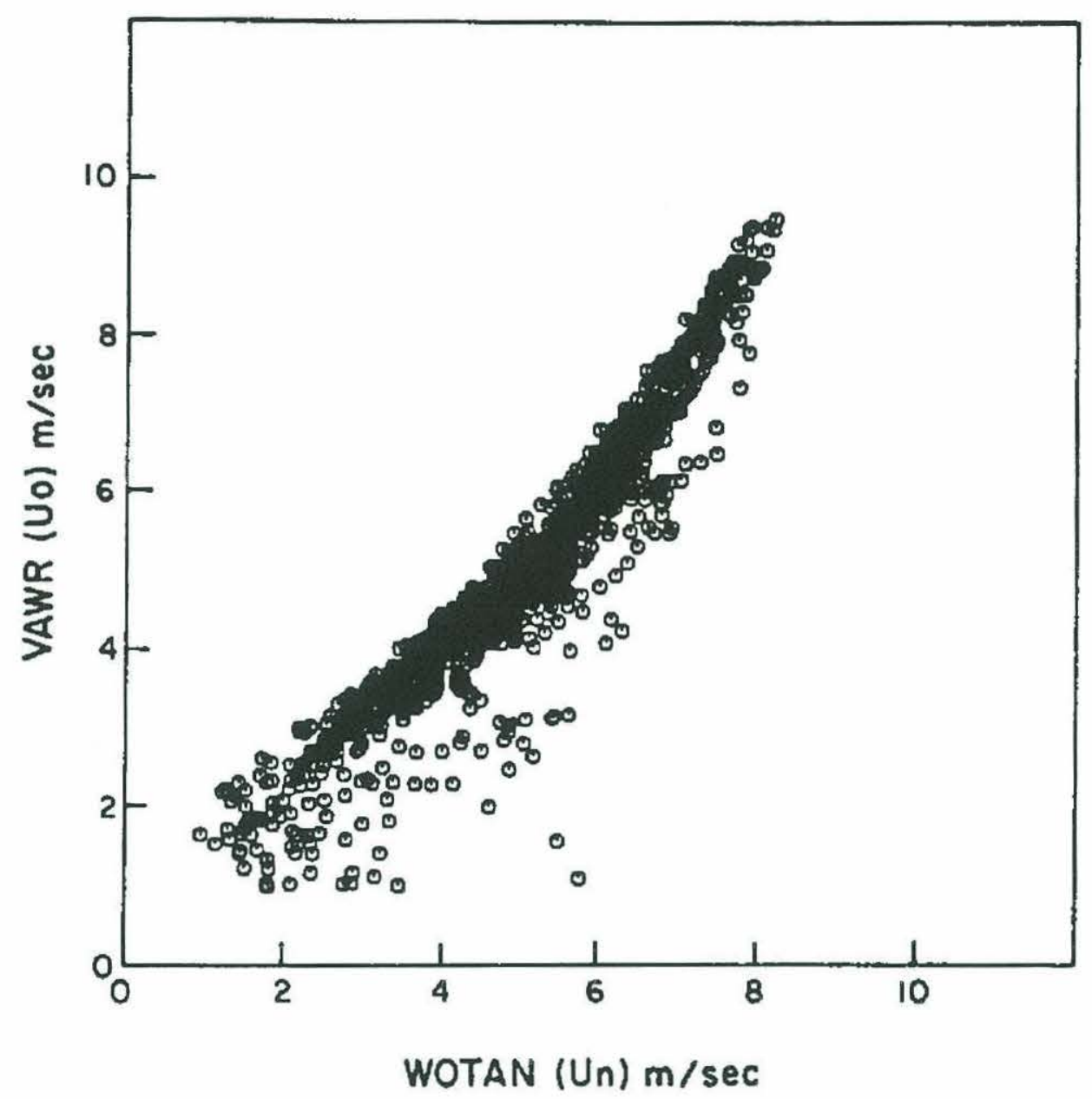

Figure 1.2. A plot comparing the wind speed measured by a vector-averaging wind recorder (VAWR) type anemometer 3.5 meters above the sea surface and by a WOTAN at $4.3 \mathrm{kHz}$ ambient noise frequency (Evans et al, 1984). 

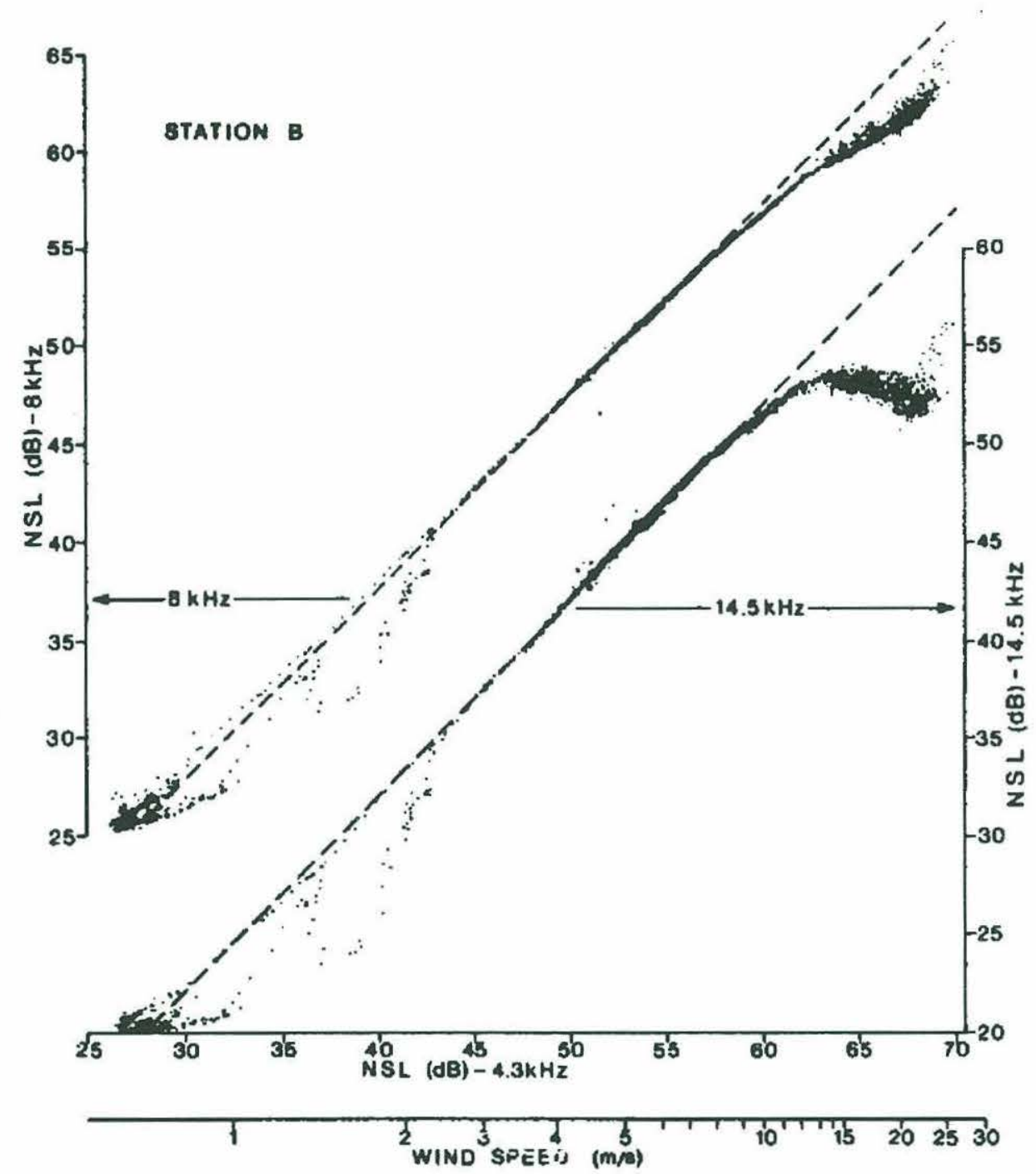

Figure 1.3. Plot of log U versus the ambient noise level at 8 and $14.5 \mathrm{kHz}$. Note that when $U$ exceeds 10 meters/second, the departure from the linear relationship between $\mathrm{N}$ and $\log \mathrm{U}$ becomes significant (Farmer \& Lemon, 1984). 
1.1 is not in dimensionless form. Crouch \& Burt obtained values of $n(f)$ ranging from 0.8 to 2.8 . By fitting data obtained by other investigators [Cato (1976), Shooter \& Gentry (1981), and Burgess \& Kewley (1983)], Urick (1986) obtained values of $n$ ranging from 0.6 to 1.5 . The differences in $\mathrm{n}$ may be due to the differences in the way the investigators sampled the data, the site dependence of the U vs. N relationship, and the differences in the sensitivity of $\mathrm{N}$ at each frequency to changes in wind speed. The degree of scatter in the data appears to be larger at the lower frequencies $[\mathrm{f}=\mathrm{O}(100 \mathrm{~Hz})]$ and the wind dependence at the higher frequencies $[\mathrm{f}>\mathrm{O}(1 \mathrm{kHz})]$ appears to be stronger.

In the last decade, the relationship between ambient noise and wind speed has been used as a method of predicting wind speed from ambient noise. Field measurements by Shaw, Watts \& Rossby (1978) showed that this relationship, described by an expression of the form

$$
\log \mathrm{U}=\mathrm{m}(\mathrm{f}) \mathrm{N}(\mathrm{f})+\mathrm{n}(\mathrm{f})
$$

can be used as a means of measuring the wind speed using hydrophones. In Equation 1.3, $\mathrm{U}$ is the wind speed in meters per second, $\mathrm{f}$ is the frequency in Hertz, $\mathrm{m}(\mathrm{f})$ and $\mathrm{n}(\mathrm{f})$ are coefficients, and the noise spectrum level $\mathrm{N}(\mathrm{f})$ is in $\mathrm{dB}$ re $1 \mu \mathrm{Pa}^{2} / 1 \mathrm{~Hz}$. This technique, commonly referred to as Weather Observations Through Ambient Noise or WOTAN (Evans, Watts, Halpern \& Bourassa, 1984), has been used in several measurements over the last decade [Evans et al (1984), Lemon, Farmer \& Watts (1984) and Vagle, Large \& Farmer (1990)]. The development of WOTAN instruments is described by Hill (1984). In the subsequent discussion in this work, equations relating $\mathrm{U}$ and $\mathrm{N}$ in the form of Equation 1.3 will be referred to as 'WOTAN equations'. Figure 1.2 shows a figure taken from Evans et al (1984) which compares wind speed 
Table 1.2. Some coefficients for the WOTAN expression and the equivalent power laws obtained from previous field measurements.

$$
\log \mathrm{U}=\mathrm{m}(\mathrm{f}) \mathrm{N}(\mathrm{f})+\mathrm{n}(\mathrm{f}) \quad \frac{\mathrm{p}^{2}}{\mathrm{p}_{\text {ref }}^{2}}=m \mathrm{U}^{n}
$$

\begin{tabular}{|c|c|c|c|c|}
\hline $\mathrm{f}(\mathrm{kHz})$ & $\mathrm{m}$ & $\mathrm{n}$ & $10^{-3} \mathrm{~m}$ & $n$ \\
\hline
\end{tabular}

Evans, Watts, Halpern \& Bourassa (1984)

\begin{tabular}{|c|c|c|c|c|}
\hline 4.3 & 0.0416 & -1.497 & 4.0 & 2.4 \\
\hline 8.0 & 0.0419 & -1.329 & 1.5 & 2.4 \\
\hline 14.5 & 0.0434 & -1.217 & 0.64 & 2.3 \\
\hline
\end{tabular}

Lemon, Farmer \& Watts (1984) site A

\begin{tabular}{|c|l|l|l|l|}
\hline 4.3 & 0.0336 & -1.045 & 1.29 & 3.0 \\
\hline 8.0 & 0.0330 & -0.925 & 0.64 & 3.0 \\
\hline 14.5 & 0.0341 & -0.810 & 0.38 & 2.9 \\
\hline
\end{tabular}

Lemon, Farmer \& Watts (1984) site B

\begin{tabular}{|c|c|c|c|c|}
\hline 4.3 & 0.0448 & -1.64 & 4.6 & 2.2 \\
\hline 14.5 & 0.0465 & -1.32 & 0.69 & 2.2 \\
\hline
\end{tabular}

Vagle, Large \& Farmer (1990)

8.0

0.0378

0.46

2.7

measurements made by WOTANs and by vector averaging wind recorders (VAWR) 3.5 meters above the sea surface. The plot shows good agreement between the two.

Table 1.2 summarizes the coefficients $\mathrm{m}$ and $\mathrm{n}$ in Equation 1.3 obtained by several investigators. Their studies confirmed the validity of Equation 1.3 but showed that $\mathrm{m}$ and $\mathrm{n}$ are site and frequency dependent. The table shows that although the regression of $\log \mathrm{U}$ and $\mathrm{N}$ is linear, there can be significant differences in the equation of the 
regression lines among different sites. In particular, the ambient noise measurements reported by Lemon et al (1984) and given in Table 1.2 were both in Queen Charlotte Sound on the west coast of Canada yet their WOTAN Equation coefficients are very different. The water depth at the measurement sites are comparable ( 265 meters at Site A and 287 meters at site B). Site A is close to the shore while site B is at the center of the channel. This suggests that the surrounding topography has a significant impact on either the noise generating mechanisms or on how the sound reaches the hydrophone.

It is important to point out however that although the WOTAN coefficients summarized in Table 1.2 differ from site to site, they do give wind speed estimates that do not differ significantly from each other within the 4 to $12 \mathrm{~m} / \mathrm{s}$ range. This is demonstrated by Table 1.3 which shows a $\pm 0.5 \mathrm{~m} / \mathrm{s}$ difference when $U=5 \mathrm{~m} / \mathrm{s}$ and $\pm 1.5 \mathrm{~m} / \mathrm{s}$ when $\mathrm{U}=10 \mathrm{~m} / \mathrm{s}$. If we restrict ourselves to the $4.3 \mathrm{kHz}$ data, the difference at $10 \mathrm{~m} / \mathrm{s}$ is less than $\pm 1.0 \mathrm{~m} / \mathrm{s}$. The error is more substantial at higher $U$ and higher frequencies.

Sound absorbed by bubbles generated by breaking waves has been shown to cause significant departures from linearity at higher ambient noise frequencies and higher wind speeds. Figure 1.3 shows a plot of $\log \mathrm{U}$ versus $\mathrm{N}$ at 8 and $14.5 \mathrm{kHz}$ measured by Farmer \& Lemon (1984) at Queen Charlotte Sound. The figure shows that when the wind speed $U$ exceeds approximately 8 meters/second, there is a clear departure from a linear relationship between $\log U$ and N. Farmer \& Lemon (1984) found that this discrepancy is more pronounced at higher frequencies and at higher wind speeds. They suggested that at higher wind speeds, waves break more frequently and more intensely. These breaking waves generate bubbles and the concentration of these 
Table 1.3. Some typical wind speeds predicted by the WOTAN equation and coefficients given in Table 1.2.

\begin{tabular}{|c|c|c|c|}
\cline { 2 - 4 } \multicolumn{1}{c|}{} & \multicolumn{3}{c|}{ U (meters/second) } \\
\hline $\mathrm{N}(4.3 \mathrm{kHz})$ & Evans et al(1984) & LFW Site A & LFW Site B \\
\hline 53 & 5.1 & $5.4 \mathrm{~m} / \mathrm{s}$ & $5.4 \mathrm{~m} / \mathrm{s}$ \\
\hline 60 & 10.0 & 9.4 & 11.1 \\
\hline 64 & 14.6 & 12.7 & 16.9 \\
\hline
\end{tabular}

\begin{tabular}{|c|c|c|c|}
\hline $\mathrm{N}(8.0 \mathrm{kHz})$ & Evans et al(1984) & LFW Site A & Vagle et al (1990) \\
\hline 48 & 4.8 & 4.6 & 6.4 \\
\hline 56 & 10.4 & 8.4 & 12.9 \\
\hline 60 & 15.3 & 11.4 & 18.3 \\
\hline
\end{tabular}

\begin{tabular}{|c|c|c|c|}
\hline $\mathrm{N}(14.5 \mathrm{kHz})$ & Evans et al(1984) & LFW Site A & LFW Site B \\
\hline 44 & 4.9 & 4.9 & 5.3 \\
\hline 51 & 9.9 & 8.5 & 11.3 \\
\hline 55 & 14.8 & 11.6 & 17.3 \\
\hline
\end{tabular}

LFW - Lemon, Farmer \& Watts (1984)

$\mathrm{N}(\mathrm{f})$ is in $\mathrm{dB}$ re $1 \mu \mathrm{Pa}^{2} / 1 \mathrm{~Hz}$

bubbles increases with increasing wind speed. These bubbles persist near the ocean surface and absorb the sound generated by breaking waves.

Vagle et al (1990) examined whether WOTAN coefficients obtained at one site can be used to predict the wind speed at other sites. They compared the WOTAN coefficients obtained during the Frontal Air-Sea Interaction Experiment (FASINEX) at a deep water site $500 \mathrm{~km}$ southwest of Bermuda with the wind and ambient noise data obtained at three shallow water and one deep water site. They concluded that the FASINEX WOTAN equations for ambient noise less than $8 \mathrm{kHz}$ can reliably predict the wind speed from the ambient noise at another deep water site as long as the 


\section{Chapter 1}

instruments are carefully calibrated and extraneous noise is screened out. They also concluded that the variations in the WOTAN equations at sites close to the shore such as those reported by Lemon et al (1984) were due to the effect of wind direction on fetch (and consequently the number of breaking waves), the effect of the bottom, the geometry of the surrounding topography, and the influence of industrial, shipping and other man-made noise.

Although the WOTAN expression is dimensionally inhomogeneous, we can nonetheless rearrange Equation 1.2 to relate $\mathrm{U}$ and $\mathrm{N}$ in terms of a power law of the form

$$
\frac{\mathrm{p}^{2}}{\mathrm{p}_{\mathrm{ref}}^{2}}=m \mathrm{U}^{n}
$$

where $m=10^{-\mathrm{n} / 10 \mathrm{~m}}$ and $n=1 /(10 \mathrm{~m})$. Based on the values of Table 1.1, we see that

$$
\mathrm{p}^{2} \sim \mathrm{U}^{2-3}
$$

\subsection{Ambient noise and the wave field.}

Although the linear relationship between $\mathrm{N}(\mathrm{f})$ and $\log \mathrm{U}$ is well established by decades of measurements, the physics behind this correlation cannot be gleaned from the WOTAN equations alone. Theoretical studies and laboratory experiments on bubbles and bubble clouds, which are reviewed in the next section, have led to a growing consensus that the sound generated by breaking waves is responsible for the wind dependence of ambient sound in the Knudsen range. However, attempts at establishing a direct and predictable relationship between ambient noise and surface 
Table 1.4. Correlation coefficients between $\mathrm{N}, \mathrm{H}_{\mathrm{s}}$ and U. (Penhallow \& Dietz, 1964)

\begin{tabular}{|c|c|c|c|c|c|c|}
\hline & \multicolumn{2}{|c|}{ steady winds } & \multicolumn{2}{c|}{ decreasing winds } & \multicolumn{2}{c|}{ increasing winds } \\
\hline & $\mathrm{U}$ & $\mathrm{H}_{\mathrm{s}}$ & $\mathrm{U}$ & $\mathrm{H}_{\mathrm{S}}$ & $\mathrm{U}$ & $\mathrm{H}_{\mathrm{s}}$ \\
\hline $\mathrm{N}(630 \mathrm{~Hz})$ & 0.90 & 0.90 & 0.80 & 0.66 & 0.79 & 0.42 \\
\hline $\mathrm{U}$ & & 0.88 & & 0.67 & & 0.47 \\
\hline
\end{tabular}

wave field parameters have been few and largely unsuccessful. Perrone (1969) found that the correlation coefficient

$$
\begin{gathered}
C_{x y}=\frac{\sum\left(x_{i}-\bar{x}\right)\left(y_{i}-\bar{y}\right)}{\sqrt{\sum\left(x_{i}-\bar{x}\right)^{2}} \sqrt{\sum\left(y_{i}-\bar{y}\right)^{2}}} \\
\bar{x}=\frac{1}{M} \sum_{i=1}^{M} x_{i} \quad \bar{y}=\frac{1}{M} \sum_{i=1}^{M} y_{i}
\end{gathered}
$$

between $\mathrm{U}$ and $\mathrm{N}$ is 0.80 while the correlation coefficient between the significant wave height $\mathrm{H}_{\mathrm{s}}$ and $\mathrm{N}$ is only 0.60 in the $200 \mathrm{~Hz}-2.8 \mathrm{kHz}$ frequency band. Farmer \& Lemon (1984) obtained comparable correlation coefficients for $\mathrm{H}_{\mathrm{s}}$ and ambient noise at 4.3, 8.0 and $14.5 \mathrm{kHz}$. By classifying measured wind velocities as steady, increasing and decreasing, Penhallow \& Dietz (1964) found that although U tracked ambient noise well under all these conditions, $\mathrm{H}_{\mathrm{s}}$ correlated well only under steady conditions. Table 1.2 summarizes their results. [Both Perrone (1969) and Penhallow \& Dietz (1964) computed the linear correlation coefficient between $N(f)$ and $U$ instead of log U.] Perhaps due to the relatively poor correlation between $\mathrm{N}$ and $\mathrm{H}_{\mathrm{s}}$, none of these studies published the regression coefficients between the two variables. 
The poor correlation between $\mathrm{N}$ and $\mathrm{H}_{\mathrm{S}}$ may appear surprising given that the ambient noise sources are expected to be direct products of the wave field itself. One should then expect that the correlation with wave parameters should be better than the wind speed correlation. This apparent discrepancy may partly be explained by the relatively large distance between the wave, wind and $\mathrm{N}$ instruments in the above experiments. Perrone's (1969) wave and wind measurements were made 30 miles from the hydrophone site. Farmer \& Lemon (1984) deployed their wave buoy 'near' their WOTAN although it is not clear what the exact distance is. Penhallow \& Dietz (1964), who made measurements close to shore, had the anemometers and wave gauges installed off a dock while the hydrophone was at 12 meters depth, 300 meters offshore.

Perhaps the more plausible explanation is that the presence of swell increases $\mathrm{H}_{S}$ without proportionally increasing the incidence of wave breaking. Since swell is generated by storms far from the observation site, it is possible, particularly in the open ocean with large fetch lengths, for $\mathrm{H}_{\mathrm{S}}$ to be large even on a calm day with low wind speeds. Some recent studies [(Thorpe, 1992) and (Melville, 1992)] suggest that it is the wind waves at frequencies greater than the wind sea spectral peak that are breaking. If their models are correct, then wave components, including wind waves and swell, which do not break make a significant contribution to the variance of the sea surface displacement but not to the noise. If wave breaking is indeed primarily responsible for the ambient noise in the ocean, then correlations with $\mathrm{N}$ should be made with wave parameters that quantify wave breaking. 


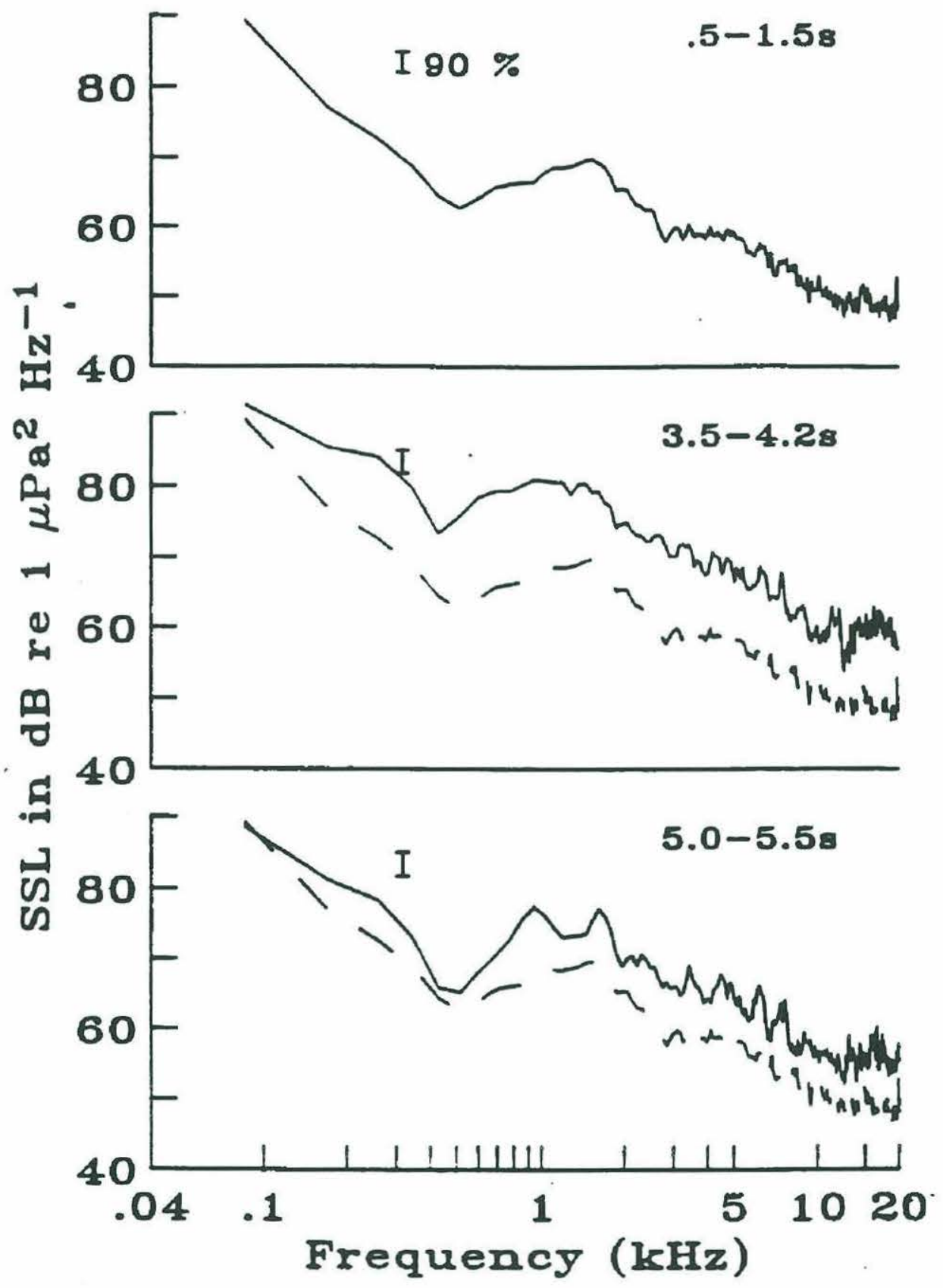

Figure 1.4. Ambient noise spectrum level during a breaking event detected by a hydrophone 14 meters below sea level. The background spectrum immediately before breaking shown in the first plot is reproduced by the dashed lines in the second and third plots. The $90 \%$ confidence level is indicated by the vertical bars in each plot (Farmer \& Vagle, 1989). 


\subsection{Sound generated by breaking waves.}

In the last decade, there have been a significant number of investigations that have looked into the acoustic properties of breaking waves. There appears to be a growing consensus that the prevailing noise level in the Knudsen range is primarily due to the wave breaking mechanisms associated with bubble creation, coalescence, splitting and collective bubble cloud oscillation.

Farmer \& Vagle (1989) described experiments off Vancouver Island in $140 \mathrm{~m}$ of water and at a site $500 \mathrm{~km}$ southwest of Bermuda in $4000 \mathrm{~m}$ water. By comparing video recordings with simultaneous acoustic measurements, they demonstrated that breaking waves can generate ambient noise at frequencies as low as $100 \mathrm{~Hz}$. Figure 1.4 , taken from their paper, shows that at frequencies within the Knudsen range, breaking waves can generate sound levels significantly higher than the background level. Hollett (1989) reported measurements of breaking wave generated noise as low as $30 \mathrm{~Hz}$ in the Mediterranean Sea.

By comparing the WOTAN measurements in Georges Strait, British Columbia at 4.3 $\mathrm{kHz}$ with a model of sound generation by randomly distributed breaking waves, Farmer \& Vagle (1988) showed that changes in the dominant wave period, the mean breaker spacing, and the mean breaking wave intensity can be inferred from changes in the ambient noise spectrum level. Their model also suggests that the underwater ambient noise level is proportional to the frequency and intensity of wave breaking in the vicinity of the hydrophone. The importance of their work is that it suggests that information about the characteristics of the wavefield and the distribution of breaking waves can be obtained from measurements of ambient sound. 


\section{Chapter 1}

Several theories on how breaking waves generate sound have been proposed [see Kerman (1984) for a review of early theories]. These include splashing water droplets [(Franz, 1959) and Wilson (1983)], cavitation noise (Furduev, 1966), nonlinear interaction of water waves [Longuet-Higgins (1951), Marsh (1963), and Kuo (1968)], and atmospheric turbulence (Fitzpatrick \& Strasberg, 1956). Kerman (1984) reviewed these theories and discussed some of their major shortcomings.

Although the oscillation of bubbles generated by breaking waves has been considered as a plausible ambient noise mechanism for several decades (Wenz, 1962), more recent laboratory experiments and theoretical modeling have suggested that this is the dominant mechanism responsible for the characteristics of wind-dependent ambient noise at higher frequencies ( $\mathrm{f}>500 \mathrm{~Hz}$ ). In addition, other recent investigations reviewed later in this section suggest the possibility that the collective oscillation of a breaking wave bubble cloud could be responsible for a significant proportion of the noise spectrum level below $500 \mathrm{~Hz}$. An extensive survey of the current research on wave breaking sound sources and mechanisms can be found in the proceedings of the 1987 NATO workshop in Lerici, Italy (Kerman, 1988) and the 1990 Conference on the Natural Physical Sources of Underwater Sound in Cambridge, UK (Kerman, 1992).

In a now classic paper, Minnaert (1933) studied the sound generated by the resonant volumetric oscillation of a newly created bubble in water. He showed that if adiabatic compression is assumed, then the resonant frequency $f_{o}$ of this mode of oscillation is given by

$$
\mathrm{f}_{\mathrm{o}}=\frac{1}{2 \pi \mathrm{r}_{\mathrm{o}}} \sqrt{\frac{3 \gamma \mathrm{P}}{\rho_{\mathrm{w}}}}
$$


where $\mathrm{r}_{\mathrm{o}}$ is the mean radius of the bubble, $\gamma=c_{p} / c_{v}$ is the ratio of the specific heats of the entrained gas, $\mathrm{P}$ is the ambient pressure at the bubble and $\rho_{\mathrm{w}}$ is the density of water. For an ideal gas under adiabatic conditions, $\gamma=1.4$.

In linear wave theory, the fluctuations in the sound pressure $\mathrm{p}$ is related to the velocity of the fluid particles $v$ by the equation (Urick, 1975, p. 12)

$$
\mathrm{p}=\rho \mathrm{cv}
$$

where $\rho$ is the density of the fluid and $c$ is the propagation velocity of the pressure wave. The value of the specific acoustic impedance $\rho c$ in sea water is $1.5 \times 10^{5} \mathrm{~g} / \mathrm{cm}^{2} \mathrm{~s}$ and $42 \mathrm{~g} / \mathrm{cm}^{2} \mathrm{~s}$ for air. The large difference in $\rho c$ between air and water implies that for an underwater sound wave, the pressure is essentially zero $(p=0)$ at the air-water interface (pressure release condition).

The resonant frequency of a bubble near the water surface is modified by the pressure release condition at the air-water interface. For a wave incident on the air-sea interface, the phase of the reflected acoustic energy is shifted by $180^{\circ}$ to satisfy the pressure release condition. A bubble oscillating near an acoustically smooth interface as a monopole at a depth $\mathrm{L} / 2$ below the water surface creates an image monopole source L/2 above the water surface oscillating out of phase with the real bubble. In short, the far field behavior of a bubble near the sea surface can be represented by a dipole whose resonant frequency is higher than that of the bubble resonating as a monopole. If the bubble radius $r_{o}<<L$, then the resonant frequency of the dipole $f_{d}$ can be approximated by (Longuet-Higgins, 1990)

$$
\mathrm{f}_{\mathrm{d}}=\mathrm{f}_{\mathrm{o}} \sqrt{1+\mathrm{r}_{\mathrm{o}} / \mathrm{L}} .
$$




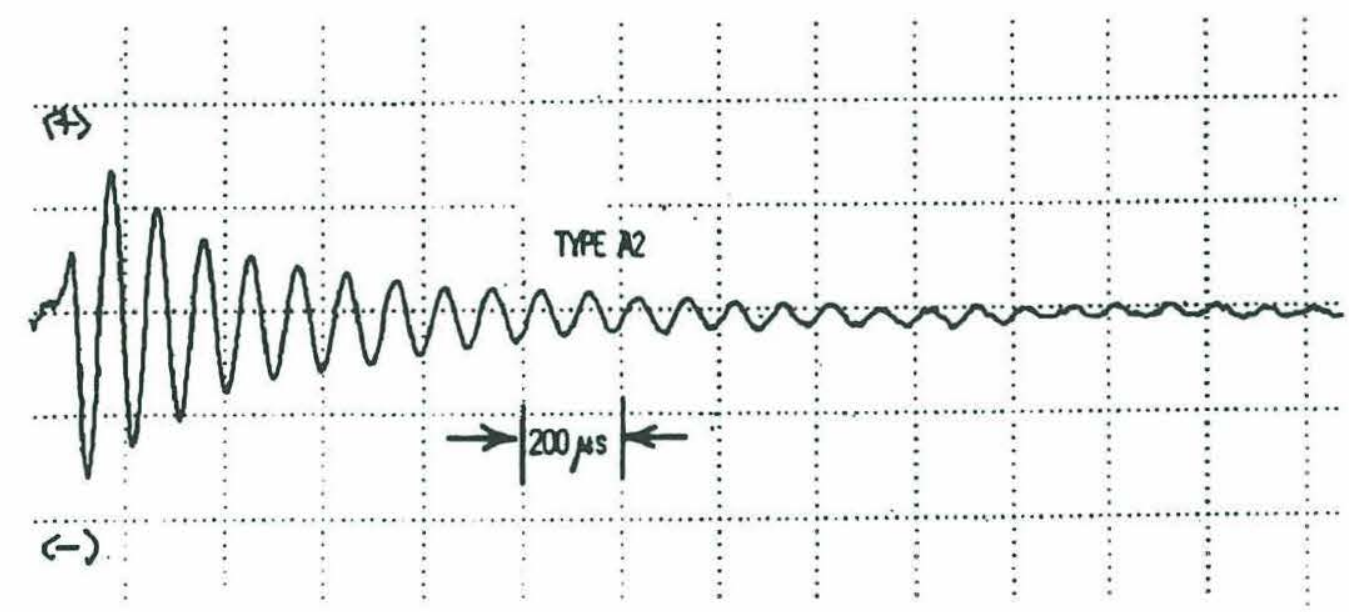

Figure 1.5. The sound radiated by a typical oscillating bubble generated by a laboratory spilling breaker can be described as an exponentially damped sinusoid (Medwin \& Beaky, 1989). 
From observations of quasi-steady spilling wave breakers in the laboratory using a high speed movie camera synchronized with the soundtrack, Banner \& Cato (1988) found that the noise bursts detected by a hydrophone corresponded to the bubble formation at the leading edge of the spill, the impact and coalescence of bubbles, and the splitting of bubbles. They also suggested that the range of sound frequencies they detected corresponded to the resonant frequencies of the range of bubble sizes they observed. A more careful experiment by Pumphrey \& Ffowcs Williams (1990) who studied the free oscillations of individual bubbles generated by water spilling from a trough and by air forced through a submerged hypodermic needle showed results that are consistent with Banner \& Cato's (1988) experiments. Laboratory measurements of spilling breakers in an anechoic tank (Medwin \& Beaky, 1989) showed that the oscillation of these bubbles can be characterized as damped sinusoids (Figure 1.5) which suggests that the bubbles were forced initially and then oscillated freely. Medwin \& Beaky (1989) then proposed that the sound from a breaking wave is due to the incoherent addition of the sound generated by the individual bubbles generated by the breaking wave.

Using Medwin \& Daniel's (1990) measurements of bubble size distribution in laboratory breaking waves and by assuming that the oscillating bubbles radiate acoustic pressure as a dipole, Loewen \& Melville (1991b) proposed a semi-empirical sound source model for gently spilling waves that closely predicted the spectral magnitude and shape of the measured sound spectrum. The largest bubble Medwin \& Daniel (1990) observed had a $7.4 \mathrm{~mm}$ radius and a $440 \mathrm{~Hz}$ resonant frequency. Loewen \& Melville (1991b) speculated that bigger bubbles may be generated by breaking waves in the ocean and these can radiate sound at even lower frequencies. 
For a large bubble cloud generated by intense breaking waves, it may be appropriate to treat it is a fluid continuum whose density is comparable to the surrounding fluid but whose compressibility is significantly higher. Carey \& Browning (1988) suggested that the oscillation of such a bubble cloud, whose dimensions are significantly larger than the largest individual bubbles in the ocean, might account for the low frequency wind-dependent noise. In a subsequent paper, Carey \& Fitzgerald (1992) showed that a spherical collection of bubbles can oscillate at a resonant frequency

$$
\mathrm{f}_{\mathrm{o}}=\frac{1}{2 \pi \mathrm{r}_{\mathrm{o}}} \sqrt{\frac{3 \gamma \mathrm{P}}{\rho_{\mathrm{w}} f}}
$$

where $r_{o}$ is the radius of the bubble cloud, $\gamma=1$ is the ratio of specific heats under isothermal conditions, $\mathrm{P}$ is the ambient pressure, $\rho_{\mathrm{w}}$ is the density of surrounding fluid, and $f$ is the mean void fraction of the bubble cloud. Note that this equation is similar to the Minnaert (1933) formula for the resonant oscillation of spherical bubbles (Equation 1.6). For a spherical bubble cloud in water of radius $r_{o}=0.1$ meters, $f=10 \%$ air, and an ambient pressure $\mathrm{p}=101 \mathrm{kPa}$, Equation 1.9 predicts a resonant frequency $\mathrm{f}_{\mathrm{o}}=90 \mathrm{~Hz}$. If the bubble cloud has a radius $\mathrm{r}_{\mathrm{o}}=1$ meter, $f=1 \%$ air, Equation 1.9 predicts a resonant frequency $\mathrm{f}_{\mathrm{o}}=30 \mathrm{~Hz}$.

A more general theory for sound generation by collective oscillations of bubble clouds was proposed by Prosperetti (1988) and Lu, Prosperetti \& Yoon (1990). Lu et al (1990) outlined their approach and used it to predict the resonant collective oscillation frequencies of bubbly regions of various geometries. In particular, they showed that their method successfully predicted the frequency of the resonant oscillations of a cylindrical bubble column generated in the laboratory (Yoon, Crum, Prosperetti \& Lu, 1991). 


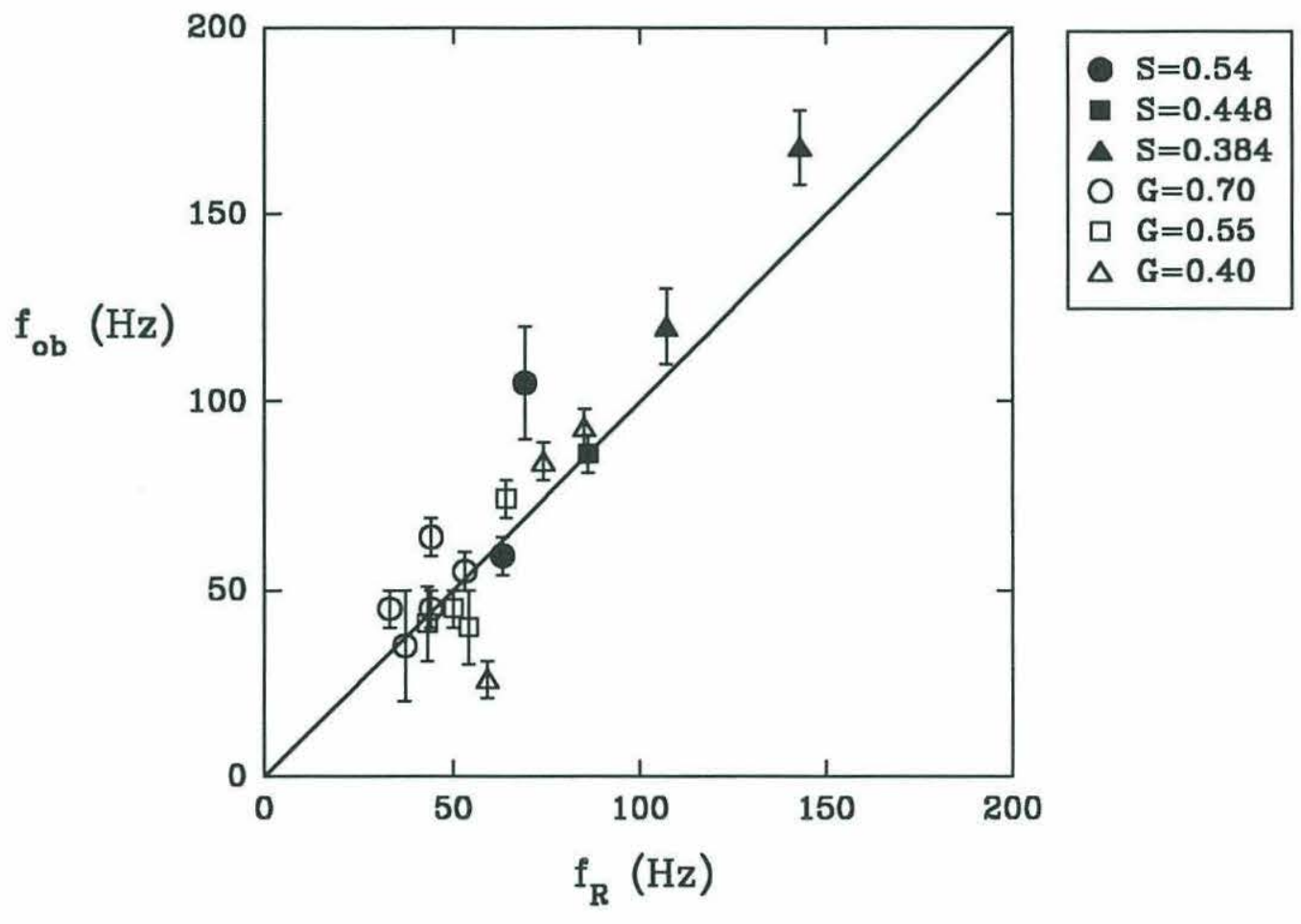

Figure 1.6. Plot showing the observed peak frequency $f_{o b}$ of the pressure spectrum from breaking waves and the predicted resonant frequency $f_{R}$ for a cylindrical bubble cloud computed using the method described by Lu et al (1990). [Loewen \& Melville, 1992]. 
Laboratory experiments using bubble columns (Yoon et al, 1991) and bubble plumes (Kolaini, Roy \& Crum, 1991) suggest that this mechanism might explain the low frequency pressure fluctuations corresponding to modes of the bubble plumes detected by a hydrophone. Kolaini et al (1991) created bubble plumes by dropping a cylindrical volume of water into a tank of water. They found that substructures consisting of a sphere of bubbly water of high void fraction break off from the main plume and oscillate at frequencies as low as tens of Hertz. The resonant frequencies they observed matched the lowest eigenfrequencies of the substructures if a void fraction of $40 \%$ within the plumes was assumed.

Recently, Loewen \& Melville (1992) [see also Loewen (1991)] observed low frequency peaks of the order of tens of Hertz in the noise spectrum of two-dimensional and three-dimensional plunging waves in the laboratory. By using the measurements of void fraction of breaking wave bubble clouds (Lamarre \& Melville, 1992) and the theory of collective bubble cloud oscillations by Lu et al (1990), they found that the low frequency peak in the spectrum of pressure fluctuations picked up by a hydrophone matches the resonant frequency of collectively oscillating bubble clouds predicted by Lu et al (1990) [see Figure 1.6].

\subsection{Breaking wave sound and dissipation.}

In the last few years, our research group has conducted a series of laboratory experiments to establish quantitative correlations between the acoustics and the dynamical characteristics of breaking waves. The relationship between the energy dissipated by breaking waves and the sound radiated was initially examined by Melville, Loewen, Felizardo, Jessup \& Buckingham (1988) for controlled breaking waves in the laboratory. This experiment, as well as the subsequent investigation by 


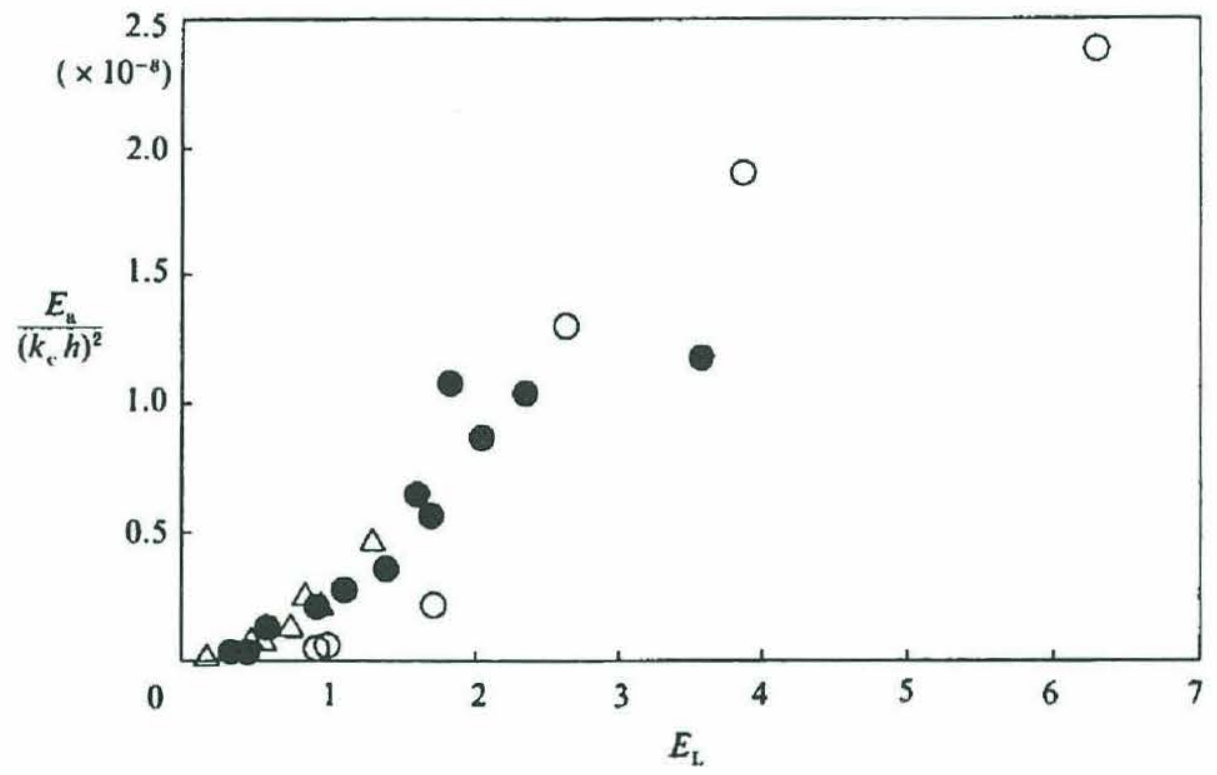

Figure 1.7. A plot showing that the acoustic energy radiated by a breaking event $\mathrm{E}_{\mathrm{a}}$ is proportional to the dissipated mechanical wave energy $\mathrm{E}_{\mathrm{L}}$. $\mathrm{E}_{\mathrm{a}}$ was scaled by the square of the product of the center wavenumber of the surface wave packet $k_{c}$ and the depth of the wave tank h (Loewen \& Melville, 1991a). 


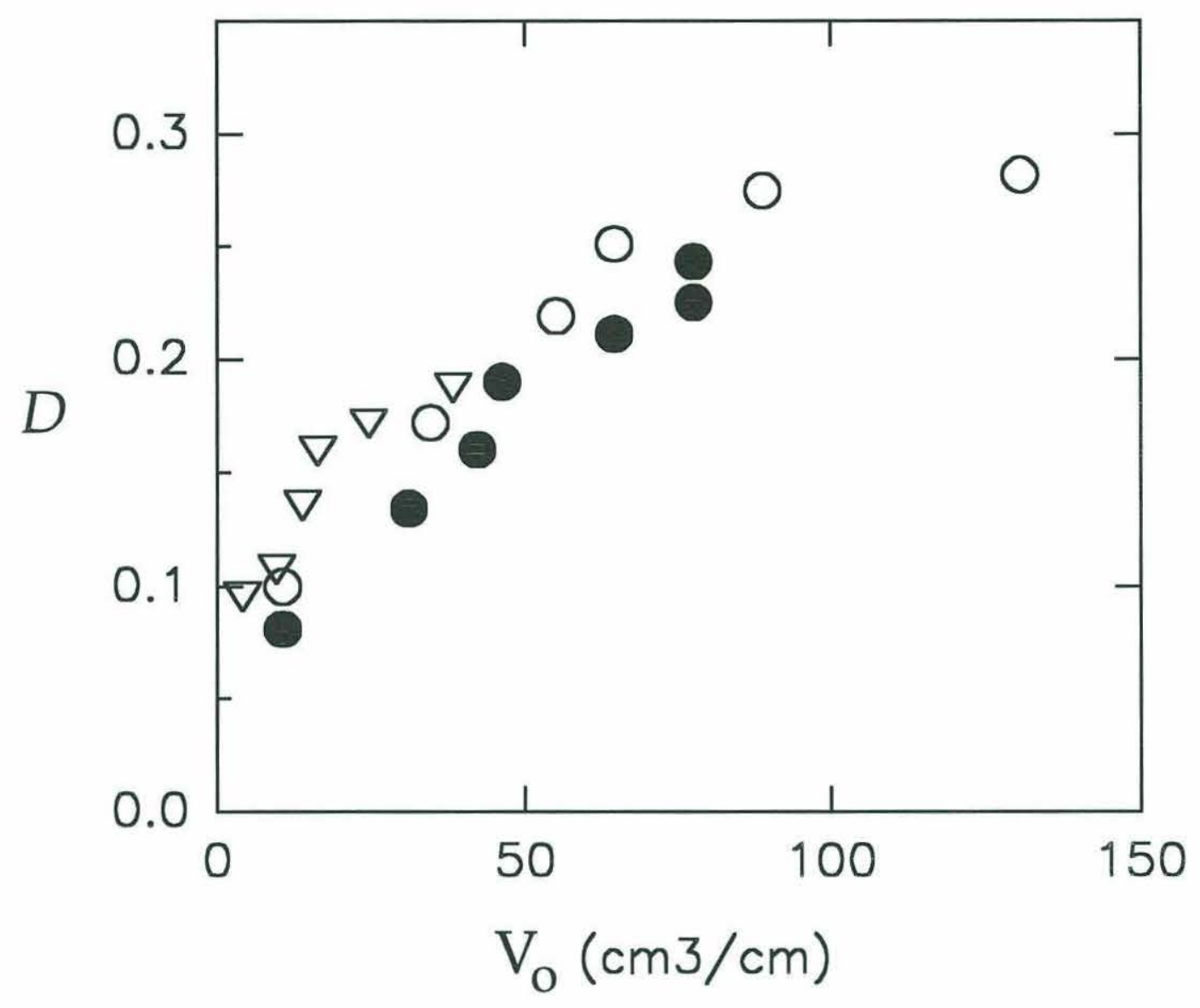

Figure 1.8. The fraction of energy dissipated by a breaking event $D$ is proportional to the initial volume per unit width of air entrained by a breaking wave $V_{o}$. The figure shows laboratory results from three different wave packets of various wave slope (adapted from Loewen, 1991). 
Loewen \& Melville (1991a) using a larger set of wave packets, established that over a limited range of parameters, the acoustic energy radiated by a breaking event is proportional to the surface wave energy dissipated by the event (Figure 1.7). In addition, Loewen \& Melville (1991a) showed that approximately $10^{-8}$ of the dissipated energy is converted to acoustic energy and that the energy of the radiated sound is proportional to the duration of the event. By relating the electrical conductivity of an air-water mixture to the void fraction of the medium, Lamarre \& Melville (1991) mapped the spatial and temporal void fraction distribution in laboratory breaking waves. By studying the evolution of the bubble cloud generated by the breaking wave, they found that a significant proportion of the dissipated energy (up to $50 \%$ ) is due to work done by the liquid in entraining air against buoyancy. Using data from similar experiments, Loewen (1991) [see also Loewen and Melville (1992)] showed that the energy dissipated by a breaking event is proportional to the initial volume of air entrained (Figure 1.8). This is consistent with the Loewen \& Melville (1991b) dipole sound source model which suggested that for similar bubble size distributions, the total acoustic energy radiated is proportional to the volume of air entrained.

These laboratory studies clearly suggest that sound generation and the dissipation of mechanical wave energy are coupled through the air entrainment process. We believe that this is the reason why the two correlate well in the laboratory and we expect that these two parameters should also correlate well in the field.

\subsection{Wind wave dissipation.}

Direct measurements of dissipation due to wave breaking in the field are not yet feasible although some progress is being made to achieve this (Agrawal et al, 1992). However, dissipation can be estimated using the energy budgets arising from wind- 


\section{Chapter 1}

wave evolution models. If terms other than dissipation can be effectively measured or modeled, then the dissipation can be inferred through an energy balance.

We can describe the distribution of the sea surface elevation $\mathrm{a}(\mathbf{x})$ using the wavenumber spectrum (Phillips, 1985)

$$
\Psi(\mathbf{k})=\frac{1}{4 \pi^{2}} \int_{\mathbf{r}} \overline{\mathrm{a}(\mathbf{x}) \mathrm{a}(\mathbf{x}+\mathbf{r})} \mathrm{e}^{-\mathbf{j} \mathbf{k} \cdot \mathbf{r}} \mathrm{d} \mathbf{r}
$$

which is given in terms of the covariance of the wave height between two points at $\mathbf{x}$ and $\mathbf{x}+\mathbf{r}$. The evolution of the surface wave field can then be represented by the energy transfer equation (Phillips, 1985)

$$
\rho_{w} g \frac{d \Psi(\mathbf{k})}{d t}=\rho_{w} g \frac{\partial \Psi}{\partial t}+\rho_{w} g\left(\mathbf{c}_{g}+\mathbf{V}\right) \cdot \nabla_{\mathbf{k}} \Psi=-\nabla_{\mathbf{k}} \cdot \mathbf{T}(\mathbf{k})+w(\mathbf{k})-d(\mathbf{k})
$$

where $\rho_{\mathrm{w}}$ is the density of water, $\mathbf{c}$ is the group velocity and $\mathbf{V}$ is the current velocity. The three forcing terms on the right hand side are the net spectral flux from resonant wave-wave interactions $-\nabla_{\mathbf{k}} \cdot \mathbf{T}(\mathbf{k})$, the spectral wind forcing term $\mathrm{w}(\mathbf{k})$, and the spectral dissipation term $\mathrm{d}(\mathbf{k})$. The net spectral flux is computed using the collision integral relating the component wavenumbers (Hasselmann, 1962, 1963)

$$
\begin{aligned}
-\nabla_{\mathbf{k}} \cdot \mathbf{T}(\mathbf{k})= & \rho_{\mathrm{w}} \iiint \mathrm{Q}^{2}\left\{\left[S(\mathbf{k})+S\left(\mathbf{k}_{\mathbf{1}}\right)\right] S\left(\mathbf{k}_{\mathbf{2}}\right) S\left(\mathbf{k}_{\mathbf{3}}\right)-\left[S\left(\mathbf{k}_{2}\right)+S\left(\mathbf{k}_{3}\right)\right] S(\mathbf{k}) S\left(\mathbf{k}_{\mathbf{1}}\right)\right\} . \\
& \times \delta\left(\mathbf{k}+\mathbf{k}_{\mathbf{1}}-\mathbf{k}_{\mathbf{2}}-\mathbf{k}_{\mathbf{3}}\right) \delta\left(\omega+\omega_{1}-\omega_{2}-\omega_{3}\right) \mathbf{d k}_{\mathbf{1}} \mathbf{d k}_{\mathbf{2}} \mathbf{d k}_{\mathbf{3}}
\end{aligned}
$$


where $S$ is the action spectral density $S(\mathbf{k})=(\mathrm{g} / \omega) \Psi(\mathbf{k})$ and $Q$ is a complicated function of order $\mathrm{k}^{3}$. This expression is clearly too complex to evaluate analytically and is computed numerically when used by wave evolution models (WAMDIG, 1988). Sell \& Hasselmann (1972), Longuet-Higgins (1976) and Fox (1976) have developed a narrow bandwidth approximation to the net spectral flux term. They found that the interactions are primarily local for the wind wave components whose amplitudes and slopes are small. Phillips (1985) then argued that Equation 1.12 behaves as

$$
\begin{gathered}
-\nabla_{\mathbf{k}} \cdot \mathbf{T}(\mathbf{k}) \sim \rho \mathrm{Q}^{2} S^{3}(\mathbf{k}) \mathrm{k}^{4} / \omega \\
\sim \rho \mathrm{gk}^{8} \Psi^{3}(\mathbf{k})
\end{gathered}
$$

By combining measurements of atmospheric pressure fluctuations over surface waves in the Bight of Abaco and Miles' (1952) wind input model, Snyder, Dobson, Elliott \& Long (1981) proposed that

$$
\mathrm{w}(\mathbf{k})=0.25 \rho_{\mathrm{a}} \mathrm{g} \max \left[0,\left(\frac{\mathrm{U}_{5}}{\mathrm{c}} \cos \theta-1\right)\right] \omega \Psi(\mathbf{k})
$$

where $\rho_{a}$ is the density of air, $U_{5}$ is the wind speed 5 meters above the mean sea level, $c$ is the phase speed of the surface wave frequency component, and $\theta$ is the angle between the wind and wave direction.

A comparable expression was proposed by Plant (1982) from a survey of wind input measurements of Kawai (1979), Snyder et al (1981), Shemdin \& Hsu (1967), Larson \& Wright (1975), Plant \& Wright (1977), Wu, Hsu \& Street (1979), and Stewart \& Teague (1980). He showed that the various wind input measurements satisfy the equation 


$$
w(\mathbf{k})=(0.04 \pm 0.02) \rho_{\mathrm{w}} g \cos \theta\left(\frac{\mathrm{u}_{*}}{\mathrm{c}}\right)^{2} \omega \Psi(\mathbf{k})
$$

where $u_{*}$ is the friction velocity of the air flow over the waves (Figure 1.9).

While the characteristics of both the spectral flux and wind input terms are clearly complex, it is generally believed that the expression for the dissipation $\mathrm{d}$ is the least understood component of the energy transfer equation. Because of the difficulty in quantifying this in the field, there have been no direct field measurements of the total surface wave dissipation. Only recently have investigators begun to make field measurements of the volumetric dissipation rate in the water column adjacent to the air-water interface.

Agrawal et al (1992) measured the surface velocity field under conditions of strong breaking from a tower in Lake Ontario. They found that the rate of dissipation of kinetic energy under those conditions can exceed the values predicted by assuming a velocity distribution based on a flat, constant stress, turbulent boundary layer or 'walllayer' theory (Tennekes \& Lumley, 1972) by two orders of magnitude. They concluded that the near-surface layer is a region of enhanced dissipation and that studies of upper mixed layer dynamics have to incorporate the influence of wave breaking explicitly.

\subsection{Breaking wave dissipation models.}

In principle, the total wave energy dissipation can be computed from the sum of the energy dissipated by all the breaking events for an area of the sea surface over a given 


\section{Chapter 1}

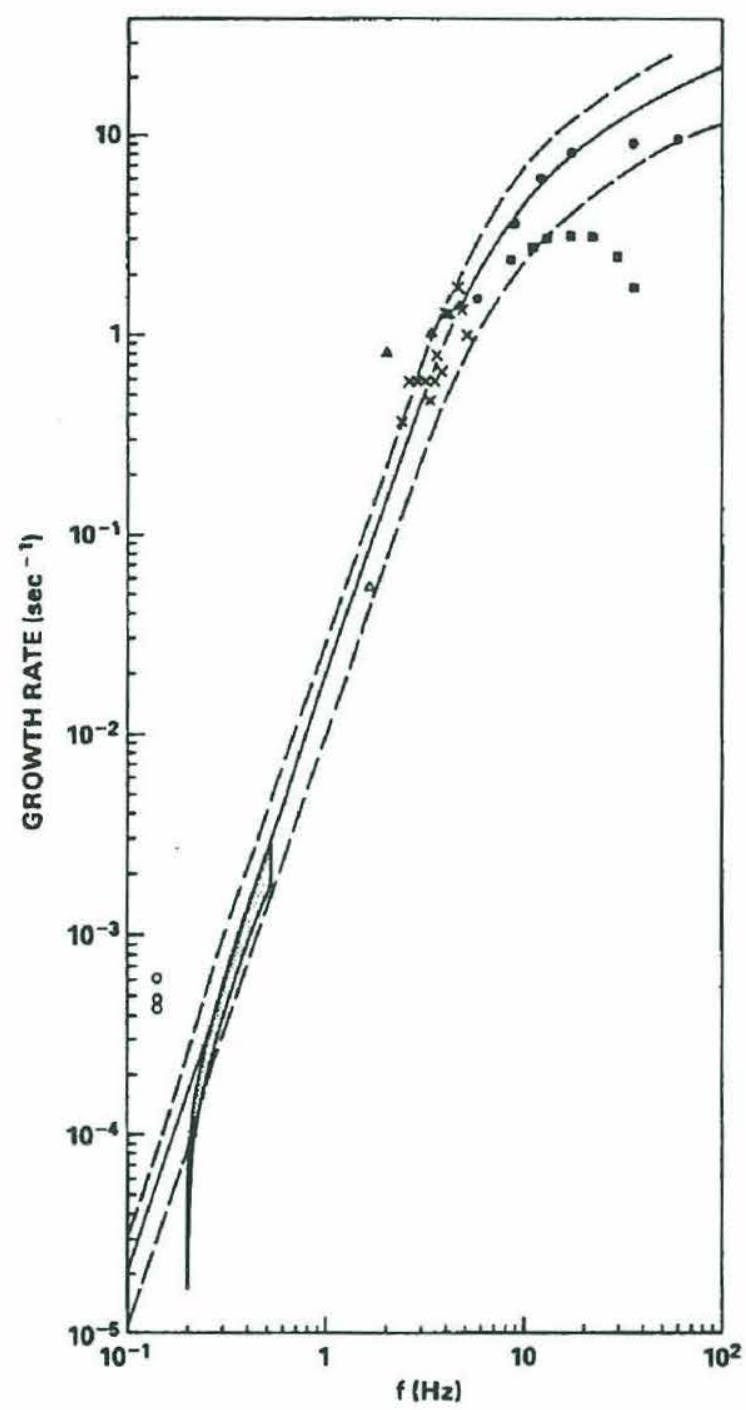

Figure 1.9. A plot of the wind induced wave growth $w / \Psi(\mathbf{k})$ as a function of frequency at $u_{*}=0.45 \mathrm{~m} / \mathrm{s}$. Data were compiled from seven different investigations by Plant (1982). Dashed lines show limits of Equation 1.14. Data from Snyder et al (1981) is indicated by shaded area (Plant, 1982). 
length of time. The difficulty in this method is in coming up with a technique that can consistently identify all the breaking events over the given area and in determining how much energy is dissipated by each of the observed events. However, this approach can be approximated by assuming that the distribution of breaking waves in the ocean is statistically stationary, and that the wind and wave conditions are quasisteady so that the percentage of wave crests that break at a given location over a finite period of time is proportional to the percentage of breaking wave crests over the sea surface at any given time.

In a recent paper, Thorpe (1992) suggested that the fraction of breaking wave crests $\xi$ is related to the inverse wave age

$$
\xi=(4 \pm 2) \times 10^{-3}\left(\frac{U_{10}}{c_{p}}\right)^{3}
$$

This equation was obtained from an empirical fit to the data from lake measurements of Thorpe \& Humphries (1980), Weissman, Atakturk \& Katsaros (1984), Atakturk \& Katsaros (1991) and Thorpe (1990). We see from Figure 1.10 that this compilation also includes two points from Longuet-Higgins \& Smith's (1983) tower measurements off the coast of Netherlands whose data actually fall above the upper limit of Equation 1.16. There is also enough scatter in the data points that they look less correlated without the dashed lines.

In any case, he assumed that on average, the energy dissipated per unit length of a breaker can be estimated from laboratory measurements of dissipation by steady breaking waves (Duncan, 1981) 


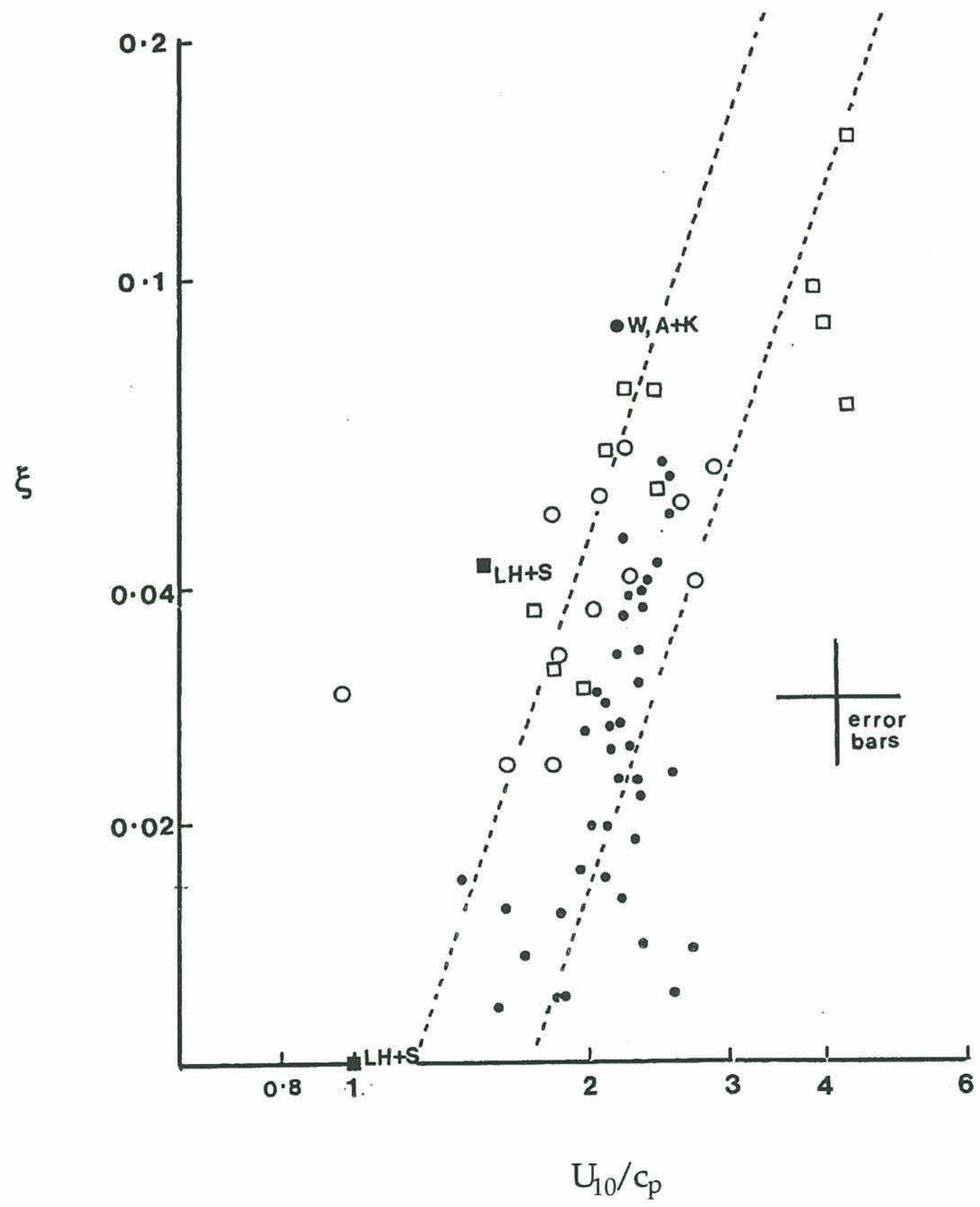

Figure 1.10. Log-log plot of the fraction of breaking waves $\xi$ versus the inverse wave age $\left(\mathrm{U}_{10} / \mathrm{c}_{\mathrm{p}}\right)$. Data was taken from various field investigations: Longuet-Higgins \& Smith (1983) - E, Weissman et al (1984) WA+K, Thorpe \& Humphries (1980) - O, Katsaros \& Atakturk (1991) - •, and Thorpe (1990) - $\square$ (Thorpe, 1992). 


$$
\mathrm{d}_{\mathrm{s}}=(0.044 \pm 0.008) \rho_{\mathrm{w}} \mathrm{c}_{\mathrm{b}}^{5} / \mathrm{g}
$$

where $c_{b}$ is the speed of propagation of the wave crest. Therefore, the total energy dissipated per unit surface area of the ocean is

$$
\begin{aligned}
\mathrm{D}_{\mathrm{T}} & =\mathrm{d}_{s} \xi / \lambda_{\mathrm{p}} \\
& =(1.9 \pm 1.1) \times 10^{-4} \frac{\rho_{\mathrm{w}} \mathrm{c}_{\mathrm{b}}^{5}}{\mathrm{~g} \lambda_{\mathrm{p}}}\left(\frac{\mathrm{U}_{10}}{\mathrm{c}_{\mathrm{p}}}\right)^{3}
\end{aligned}
$$

where $\lambda_{p}$ is the wavelength of the dominant wave. If deep water linear dispersion is assumed, $c_{p}{ }^{2}=g \lambda_{p} / 2 \pi$ and

$$
\mathrm{D}_{\mathrm{T}}=(3.0 \pm 1.8) \times 10^{-5} \rho_{\mathrm{w}} \mathrm{U}_{10}^{3}\left(\frac{\mathrm{c}_{\mathrm{b}}}{\mathrm{c}_{\mathrm{p}}}\right)^{5} .
$$

We can, in principle, measure the variables in Equation 1.19 to compute $\mathrm{D}_{\mathrm{T}}$. However, unlike the other variables on the left hand side of that equation, $c_{b}$ is difficult to measure. Although Ding \& Farmer (1992) have recently developed a method for tracking the motion of breaking waves using the phase differences in the acoustic signal received by an array of hydrophones, the value of $c_{b}$ is difficult obtain using commonly available meteorological and oceanographic instruments.

Alternatively, Thorpe (1992) suggested that if $c_{b}$ is a fraction of $c_{p}$ (or if the wave frequency components higher than the spectral peak break), we can obtain $c_{b}$ as a function of $c_{p}$ if we know the total energy dissipation $D_{T}$ in Equation 1.19. He assumed that the volumetric dissipation in the upper 5 meters is comparable to Oakey \& Elliot's (1982) measurements of the mean volumetric dissipation in the 20-meter 
upper mixed layer 5-10 meters below the sea surface. Thorpe (1992) found that if he assumed that $\left(c_{b} / c_{p}\right)^{5}=1$ in Equation 2.30, then $D_{T}$ exceeds Oakey \& Elliot's (1982) dissipation estimates by a factor of 1000 . Hence, for the two to be comparable,

$$
\begin{gathered}
\left(c_{b} / c_{p}\right)^{5}=10^{-3} \\
c_{b} \approx 0.25 c_{p} .
\end{gathered}
$$

The measurements of Agrawal et al (1992) showed that the dissipation just beneath the water surface to a depth of the order of the waveheight can be up to two orders of magnitude larger than that predicted by a turbulent boundary layer near a rigid wall. Melville (1992) proposed these results suggest the existence of a shallow layer of enhanced dissipation just beneath the sea surface. He then showed that by combining Phillips' (1985) dissipation model and measurements of turbulence and mixing by laboratory breaking waves (Rapp \& Melville, 1990), the rate of enhanced dissipation and the thickness of this layer can be estimated, and that these estimates are consistent with Agrawal et al's (1992) results.

In comparing his model with that of Thorpe (1992), Melville (1992) argued that by using Duncan's (1981) dissipation measurements of steady laboratory breaking waves, Thorpe's (1992) dissipation formula $D_{T}$ (Equation 1.19) overestimates the total energy dissipated by individual breaking events. He showed that laboratory measurements (Loewen \& Melville, 1991a) suggest that the energy dissipated by unsteady breaking waves is given by

$$
\mathrm{d}_{\mathrm{u}}=(0.0032-0.016) \rho_{\mathrm{w}} \mathrm{c}_{\mathrm{b}}^{5} / \mathrm{g}
$$


He suggested that since spilling waves constitute most of the breakers in deep water, the value of $d_{u}$ that should be used should be closer to the lower limit of Equation 1.21, approximately one order of magnitude smaller than Equation 1.17. Hence, Melville (1992) concluded that $\mathrm{D}_{\mathrm{T}}$ (Equation 1.19) overestimates the total dissipation of individual breaking waves by one order of magnitude.

Melville (1992) also disagreed with Thorpe (1992) that the total dissipation should be comparable to the upper mixed layer dissipation measured by Oakey \& Elliott (1982). Based on his estimates of enhanced dissipation in the shallow layer beneath the sea surface, Melville (1992) suggested that the dissipation rate is two orders of magnitude larger than Oakey \& Elliot's (1982) measurements over a depth one order of magnitude smaller than the upper mixed layer.

Melville (1992) proposed that since Equation 1.19 overestimates the total individual breaking wave dissipation by a factor of 10, and since using Oakey \& Elliot's (1982) results underestimates the surface wave dissipation by a factor of 10 , then the ratio $\left(c_{b} / c_{p}\right)^{5}$ in Equation 1.20 is should be 0.1. Consequently, Melville (1992) concluded that

$$
c_{b} \approx 0.63 c_{p} \text {. }
$$

This value is comparable to the results of Ding (1993) who found that the speed of breaking waves they observed is $0.4-0.7 c_{p}$ (Figure 1.11).

Both Thorpe (1992) and Melville (1992) propose methods for estimating the total dissipation from measurements of the total dissipation by breaking waves. However, their approach suffers from two major drawbacks. First, the accuracy of estimates 


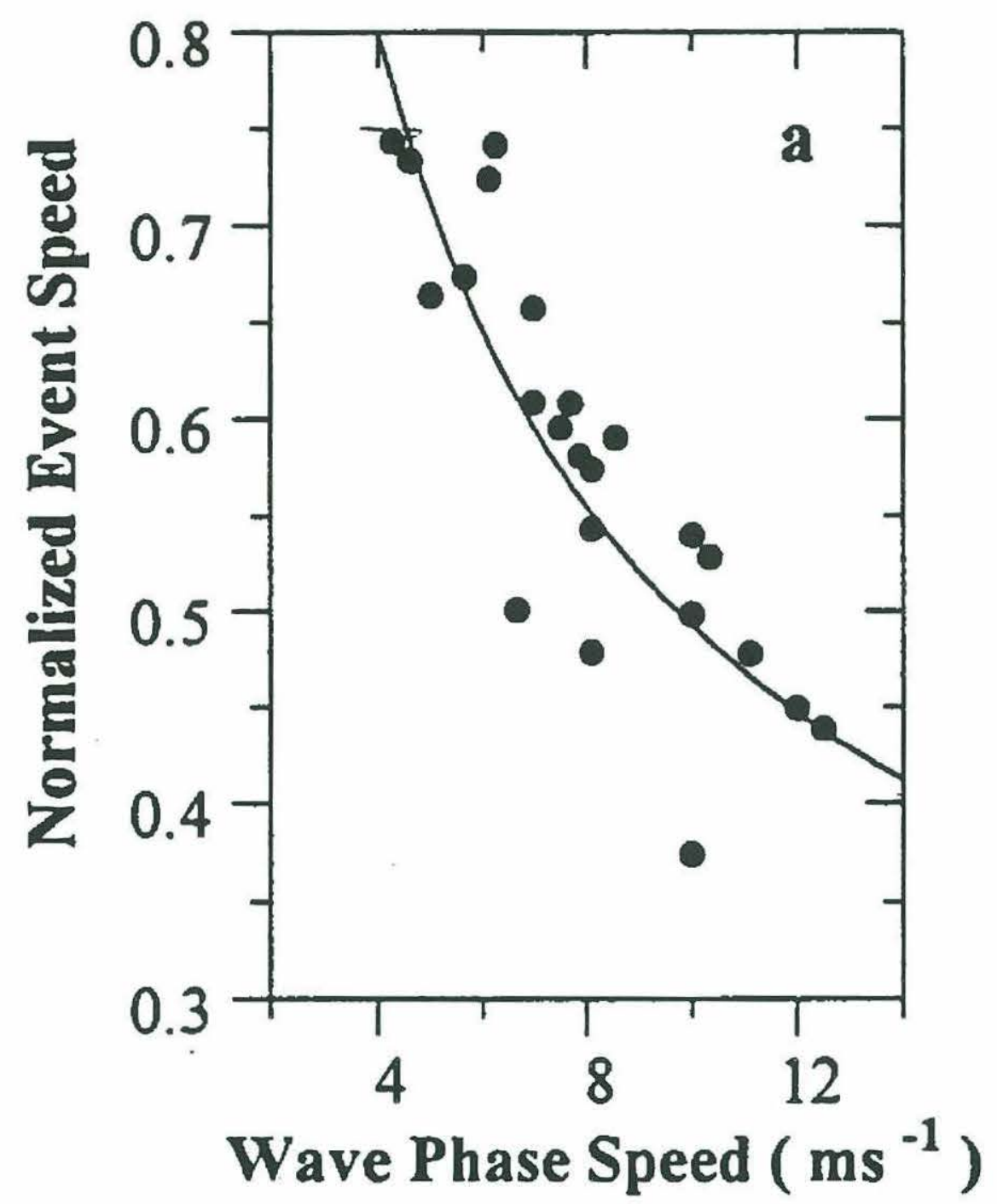

Figure 1.11. Normalized speed of a breaking event $\left(c_{b} / c_{p}\right)$ versus the phase speed $c_{p}$ (Ding, 1993). 
using their models is premised on the validity of the empirical relationship between $\xi$ and $\left(\mathrm{U}_{10} / \mathrm{c}_{\mathrm{p}}\right)$ given by Equation 1.16. Given the scatter in Figure 1.10, there is a need to obtain more breaking wave surveys to adequately justify that Equation 1.16 accurately describes this relationship. Second, since the ratio $\left(c_{b} / c_{p}\right)$ is raised to the 5 th power, then small errors in the estimate of this value will result in large differences in the total dissipation estimate. For example, the upper and lower limits of Ding's (1993) measurements of $c_{b}$ gives dissipation estimates that differ by one order of magnitude.

\subsection{Outline of thesis.}

The results of the laboratory experiments of Melville and his coworkers suggest that the ambient noise level in the ocean is related to the magnitude of the wave field characteristics that correlate with the frequency and the strength of breaking. In particular, the laboratory experiments suggest that wave energy dissipation and ambient noise generation are coupled together by the air entrainment process in breaking waves. While the dissipation of surface wave energy can be quantified in the laboratory under well-controlled conditions, as the lake experiment by Agrawal et al (1992) indicates, this cannot be easily accomplished in the field. Hence, we need to resort to models to provide us with estimates of dissipation in the ocean. In Chapter 2, we will discuss the two major dissipation models in the literature. We will then derive methods of estimating dissipation from integral characteristics of the wave field based on the assumptions of the two models. In Chapter 3, we will describe an experiment we conducted on a research platform off the coast of Oregon where we made simultaneous measurements of ambient noise, wind speed and wind direction, directional wave spectra, and video recordings of the occurrence of breaking waves. In Chapter 4, we will discuss the results of the measurements and examine the 
characteristics of the wave spectrum and how ambient noise relates to different wave field parameters. In Chapter 5, we will use the wave measurements to compute estimates of the dissipation using the dissipation models described in Chapter 2. We will then show that the relationship between wave energy dissipation and ambient noise can also be described by well-correlated power law expressions. These results are then summarized in Chapter 6 where the consequences of these results for the remote sensing of the upper ocean using ambient noise are discussed. 


\section{SURFACE WAVE DISSIPATION MODELS}

Since reliable methods for computing dissipation directly from breaking waves in the field are still currently unavailable, we adopt the alternative approach of estimating dissipation from the energy budget of a wave evolution model. This method was introduced in Section 1.5 and is discussed in detail here. In this chapter, we examine how the assumption of equilibrium of the wind input, dissipation and nonlinear spectral flux can lead to expressions for the spectral dissipation. We then show that the total dissipation can be estimated by substituting simple idealized forms of the wave spectrum into the spectral dissipation equation.

\subsection{Equilibrium spectral slopes.}

In an earlier paper, Phillips (1958) argued that in a fully developed sea, the characteristics of the wind generated waves are described by an equilibrium determined by wave breaking but independent of time and fetch. Using dimensional arguments, he concluded that the equilibrium wave height frequency spectrum is

$$
\Phi(\omega)=\mathrm{mg}^{2} \omega^{-5}
$$

where $\mathrm{m}$ is a non-dimensional constant and

$$
\Phi(\omega)=\frac{1}{2 \pi} \int_{-\infty}^{\infty} \overline{a(x, t) a(x, t+\tau)} e^{-j \omega \tau} d \tau .
$$

He also concluded that the wavenumber spectrum is 


$$
\Psi(\mathrm{k}) \sim \mathrm{F}(\theta) \mathrm{k}^{-4}
$$

where $F(\theta)$ is the directional wavenumber distribution. Observational evidence from subsequent experiments [e.g., Toba (1973), Kawai, Okuda \& Toba (1977), Forristal (1981), Donelan, Hamilton \& Hui (1984)] suggest that the form of the wave spectrum in fact depends on fetch. These investigations show that the form of the wind wave spectrum is closer to Toba's (1973) expression

$$
\Phi(\omega)=\alpha u_{*} g \omega^{-4},
$$

which he obtained from wind-wave tunnel experiments and dimensional analysis.

In the last chapter, we discussed how the energy transfer equation (Equation 1.11) describes the evolution of the surface wave field. Phillips (1985) argued that for the higher frequency components of the wave field (wind waves), the temporal and spatial scales for growth and decay are significantly longer than the internal wind wave time and length scales. From this, he assumed that these waves are essentially in equilibrium

$$
\rho_{w} g \frac{d \Psi}{d t}=-\nabla_{k} \cdot \mathbf{T}(\mathbf{k})+w(\mathbf{k})-d(\mathbf{k}) \cong 0
$$

and that the three source terms are proportional and of comparable order

$$
-\nabla_{\mathbf{k}} \cdot \mathbf{T}(\mathbf{k}) \propto \mathrm{w}(\mathbf{k}) \propto \mathrm{d}(\mathbf{k})
$$


Using these assumptions and combining the spectral flux form (Equation 1.13) and the Plant (1982) wind input expression (Equation 1.15), he showed that the equilibrium forms for the wavenumber and wave frequency spectra are

$$
\begin{gathered}
\Psi_{\mathrm{E}}(\mathbf{k})=\beta(\cos \theta)^{p} \mathrm{u}_{*} \mathrm{~g}^{-1 / 2} \mathrm{k}^{-7 / 2} \\
\Phi_{\mathrm{E}}(\omega)=\alpha \mathrm{u}_{*} \mathrm{~g} \omega^{-4}
\end{gathered}
$$

where $\alpha=0.11$ (the Toba coefficient). The value of $\beta$ is obtained from the equation $\beta=\alpha / 4 \mathrm{I}(p)$ which relates the magnitudes of the frequency and the wavenumber spectra, and the surface wave spreading function

$$
\mathrm{I}(p)=\int_{-\pi / 2}^{\pi / 2} \cos ^{p} \theta \mathrm{d} \theta
$$

For $p=0.5, \mathrm{I}(p)=2.4$ and $\beta=0.0115$

\subsection{Phillips dissipation model.}

Using the equilibrium arguments described in the last section, Phillips (1985) evaluated $\mathrm{d}(\mathbf{k})$ from Equation 2.5 using the proposed forms of the expressions for the spectral flux $\nabla_{\mathbf{k}} \cdot \mathbf{T}(\mathbf{k})$ and $\mathbf{w}(\mathbf{k})$. He showed that

$$
\begin{aligned}
& \mathrm{d}(\mathbf{k})=\rho_{\mathrm{w}} \gamma \mathrm{gk}^{-4}\left[\mathrm{k}^{4} \Psi^{3}(\mathbf{k})\right]^{3} \\
& \mathrm{~d}(\mathbf{k})=\rho_{\mathrm{w}} \gamma \mathrm{gk}^{8} \Psi^{3}(\mathbf{k})
\end{aligned}
$$


where $\gamma$ is a numerical constant. Since $\mathrm{w}(\mathbf{k})$ and $\mathrm{d}(\mathbf{k})$ are of comparable magnitude, the value of $\gamma$ can be estimated by equating dissipation with the wind input term, $\gamma \beta^{2} \approx 0.04$. Hence, $\gamma \approx 305$. To express $d(\mathbf{k})$ in terms of $\Phi$ and $\omega$, we substitute Equation 2.7 into Equation 2.10, use the linear dispersion relation $\omega^{2}=\mathrm{gk}$, and get

$$
\begin{aligned}
& \mathrm{d}=\rho_{\mathrm{w}} \mathrm{g} \gamma \mathrm{k}^{-4}\left[\beta \cos ^{p} \theta \mathrm{u}_{*} \mathrm{~g}^{-1 / 2} \mathrm{k}^{1 / 2}\right]^{3} \\
&=\rho_{\mathrm{w}} \mathrm{g} \gamma \mathrm{k}^{-4}\left[\frac{\alpha}{4 \mathrm{I}(p)} \cos ^{p} \theta \mathrm{u}_{*} \frac{\omega}{\mathrm{g}}\right]^{3} \\
&=\rho_{\mathrm{w}} \mathrm{g} \gamma \mathrm{k}^{-4}\left[\frac{\cos ^{p} \theta}{4 \mathrm{I}(p)} \frac{\omega^{5}}{\mathrm{~g}^{2}} \alpha \mathrm{u}_{*} \mathrm{~g} \omega^{-4}\right]^{3} \\
& \mathrm{~d}(\omega)=\rho_{\mathrm{w}} \mathrm{g} \gamma \mathrm{k}^{-4}\left[\frac{\cos ^{p} \theta}{4 \mathrm{I}(p)} \frac{\omega^{5}}{\mathrm{~g}^{2}} \Phi_{\mathrm{E}}(\omega)\right] .
\end{aligned}
$$

The total Phillips dissipation in the equilibrium range is

$$
\mathrm{D}_{\mathrm{P}}=2 \int_{-\frac{\pi}{2}}^{\frac{\pi}{2}} d \theta \int_{\mathbf{k}} \mathrm{k} d \mathrm{kd}(\mathbf{k})
$$

To obtain an estimate of the total dissipation based on Phillips (1985) assumptions using the measured wave spectrum, we use $\Phi(\omega)$ instead of $\Phi_{\mathrm{E}}(\omega)$ and substitute Equation 2.12 into Equation 2.13, we get an expression for the dissipation in the wind wave range from field measurements of the wave spectrum based on the Phillips (1985) assumptions

$$
\mathrm{D}_{\mathrm{P}}=\frac{\mathrm{I}(3 p) \rho_{\mathrm{w}} \mathrm{g}^{-3} \gamma}{16[\mathrm{I}(p)]^{3}} \int_{\omega_{\mathrm{p}}} \omega^{11} \Phi^{3}(\omega) d \omega
$$


The lower limit of the equilibrium range is assumed to be the spectral wind wave peak. The integral in Equation 2.14 will converge as long as the spectral slope of $\Phi(\omega)$ is steeper than $\omega^{-4}$ when $\omega \rightarrow \infty$. Note that our use of $\Phi(\omega)$ instead of $\Phi_{\mathrm{E}}(\omega)$ is a modification of Phillips' (1985) explicit assumption of an equilibrium spectrum. However, Equation 2.14 is justified if it can be demonstrated that $\Phi(\omega)$ is reasonably approximated by Phillips (1985) assumed equilibrium form of the wave frequency spectrum (Equation 2.8).

If we instead combine Equations 2.7, 2.11 and 2.13, we get Phillips' (1985) reduced dissipation estimate

$$
\begin{gathered}
\mathrm{E}_{\mathrm{P}}=2 \int_{-\frac{\pi}{2}}^{\frac{\pi}{2}} d \theta \int_{\mathrm{k}_{0}}^{\mathrm{k}_{1}} \mathrm{k} d \mathrm{k} \varepsilon(\mathbf{k}) \\
\mathrm{E}_{\mathrm{P}}=2 \gamma \beta^{3} \mathrm{I}(3 p) \rho_{\mathrm{w}} \mathrm{u}_{*}^{3} \ln \left(\frac{\mathrm{k}_{1}}{\mathrm{k}_{0}}\right) .
\end{gathered}
$$

Phillips' (1985) assumptions lead to a logarithmic increase in the dissipation with increasing wavenumber. Hence, there is a need to specify an upper spectral limit to his dissipation expression. Phillips (1985) assumed that the lower limit of the equilibrium range to be the spectral peak, $\mathrm{k}_{0}=\mathrm{g} / \mathrm{c}_{\mathrm{p}}^{2}$. He then argued that the upper limit is determined by the presence of wind drift $\mathrm{c}_{\mathrm{q}}$, which suppresses the formation of high frequency waves traveling at the same velocity (Banner \& Phillips, 1984). Using the work of Keulegan (1951) whose field measurements suggest that $c_{q} \approx u_{*}$, he chose the upper limit to be $\mathrm{k}_{1}=\mathrm{rg} / \mathrm{u}_{*}^{2}$ where $\mathrm{r}$ is a constant of order 1 . 
Chapter 2

Equation 2.16 then becomes

$$
E_{P} \approx 2 \gamma \beta^{3} I(3 p) \rho_{w} u_{*}^{3} \ln \left[r\left(\frac{c_{p}}{u_{*}}\right)^{2}\right]
$$

\subsection{Hasselmann dissipation model.}

Hasselmann (1974) argued that although wave breaking is locally a strong nonlinear process, it is in general 'weak in the mean' and its effect on the dissipation $d(\omega)$ is a quasi-linear function of $\Phi(\omega)$ and a damping coefficient proportional to the square of the frequency $\omega$, i.e.,

$$
\mathrm{d}(\omega)=-\eta \omega^{2} \Phi(\omega)
$$

He suggested that the value of the coefficient $\eta$ be obtained from an equilibrium balance of the three source terms on the right hand side of the energy transfer equation. Komen, Hasselmann \& Hasselmann (1984) [herein referred to as KHH] proposed an expression for the coefficient $\eta$ which they obtained by performing numerical simulations on the forms of $\eta$ to reproduce the characteristics of fetchlimited wave growth. They used a numerical evaluation of the full Hasselmann (1962, 1963) spectral flux equation (Equation 1.12) for $\nabla_{\mathbf{k}} \cdot \mathbf{T}(\mathbf{k})$ and the Snyder et al (1981) wind input model for w(k). A paper by the Wave Model Development and Implementation Group (WAMDIG, 1988) describes a numerical implementation of a wave development model using a numerically stable form of the $\mathrm{KHH}$ dissipation expression. While the WAMDIG (1988) expression is slightly different from the KHH formula, it is based on the same assumptions and gives dissipation estimates that are 
of the same order of magnitude as those given by the $\mathrm{KHH}$ formula. From $\mathrm{KHH}$, the total dissipation due to wave breaking is

$$
\mathrm{D}_{\mathrm{H}}=\rho_{\mathrm{w}} \mathrm{g} \int_{\omega_{o}}^{\infty} c_{H}(\omega / \bar{\omega})^{2}\left(\bar{\alpha} / \bar{\alpha}_{\mathrm{PM}}\right)^{2} \bar{\omega} \Phi(\omega) \mathrm{d} \omega
$$

where $c_{H}=3.33 \times 10^{-5}, \bar{\alpha}=\mathrm{e} \bar{\omega}^{4} / \mathrm{g}^{2}$ is a measure of the wave steepness, $\bar{\alpha}_{\mathrm{PM}}=4.57 \times 10^{-3}$ is the value of $\bar{\alpha}$ for a fully developed wind sea based on the PiersonMoskowitz spectrum, $\rho_{\mathrm{w}}=1025 \mathrm{~kg} / \mathrm{m}^{3}$ is the density of sea water, $\mathrm{e}=\int_{\omega} \Phi(\omega) \mathrm{d} \omega$ is the total surface wave energy, and

$$
\bar{\omega}=\int_{\omega} \Phi \omega \mathrm{d} \omega / \int_{\omega} \Phi \mathrm{d} \omega
$$

is the mean frequency of the wave spectrum.

Although both $\mathrm{KHH}$ and Phillips (1985) derived dissipation expressions by balancing the contributions of the three source terms, they arrived at substantially different expressions for $\mathrm{D}$ because of the differences in their assumed spectral dependence of $\mathrm{d}(\omega)$, differences in the source functions for $\mathrm{w}(\mathbf{k})$ and $\nabla_{\mathbf{k}} \cdot \mathbf{T}(\mathbf{k})$ used, and differences in their method of balancing the source terms.

\subsection{Reduced Hasselmann dissipation estimate.}

In many instances, the full wave spectrum is not available. Instead, the summary wave data is limited to a few variables, e.g., root mean square wave amplitude $a$ and the mean frequency of the wave spectrum $\bar{f}$. We can use these parameters to obtain a 
dissipation estimate by assuming that the frequency spectra can be approximated by a simple power law in terms of $\omega$. This was essentially Phillips' (1985) approach when he derived Equation 2.16 from Equation 2.11. However, his rationale was based primarily on the assumption of the existence of equilibrium spectra and is valid only in the wind wave region of the spectrum.

While the basis of the $\mathrm{KHH}$ dissipation expression is independent of the existence and form of an equilibrium spectrum, based on the discussions in Section 2.1, we nevertheless assume an idealized wave spectrum of the form

$$
\begin{aligned}
\Phi_{\mathrm{M}}(\omega) & =\mathrm{mu}_{*} \mathrm{~g} \omega^{-4} & & \omega \geq \omega_{\mathrm{p}} \\
& =0 & & \omega<\omega_{\mathrm{p}}
\end{aligned}
$$

where $\mathrm{m}$ is a constant. Both the frequency and wavenumber spectrum satisfy Parseval's theorem

$$
a^{2}=\int_{\mathbf{k}} \Psi(\mathbf{k}) \mathrm{d} \mathbf{k}_{1} \mathrm{~d} \mathbf{k}_{2}=\int_{\omega} \Phi(\omega) \mathrm{d} \omega .
$$

Substituting Equation 2.21 into Equation 2.22 and integrating gives

$$
\begin{gathered}
a^{2}=\frac{1}{3} \mathrm{mu}_{*} \mathrm{~g} \omega_{\mathrm{p}}^{-3} \\
\mathrm{~m}=\frac{3 a^{2} \omega_{\mathrm{p}}^{3}}{\mathrm{u}_{*} \mathrm{~g}} .
\end{gathered}
$$

Combining Equations 2.21 and 2.23 gives 
Chapter 2

$$
\Phi_{\mathrm{M}}(\omega)=3 a^{2} \omega_{\mathrm{p}}^{3} \omega^{-4}, \quad \omega \geq \omega_{\mathrm{p}}
$$

The relationship between $\bar{\omega}$ and $\omega_{\mathrm{p}}$ is obtained by substituting Equation 2.24 into the definition of $\bar{\omega}$ (Equation 2.20). For a spectrum with an $\omega^{-4}$ tail,

$$
\bar{\omega}=1.5 \omega_{\mathrm{p}} .
$$

Substituting Equations 2.24 and 2.25 into Equation 2.19 gives the equation for the reduced $\mathrm{KHH}$ dissipation

$$
\begin{aligned}
\mathrm{E}_{\mathrm{H}} & \equiv \rho_{\mathrm{w}} \mathrm{g} \int_{\omega_{\mathrm{p}}}^{\infty} c_{H}(\omega / \bar{\omega})^{2}\left(\bar{\alpha} / \bar{\alpha}_{\mathrm{PM}}\right)^{2} \bar{\omega}\left[0.889 a^{2} \bar{\omega}^{3} \omega^{-4}\right] \mathrm{d} \omega \\
& =0.889 \rho_{\mathrm{w}} \mathrm{g} c_{H}\left(\bar{\alpha} / \bar{\alpha}_{\mathrm{PM}}\right)^{2} a^{2} \bar{\omega}^{2} \int_{\omega_{\mathrm{p}}}^{\infty} \omega^{-2} \mathrm{~d} \omega .
\end{aligned}
$$

Integrating Equation 2.26 gives

$$
\mathrm{E}_{\mathrm{H}}=1.333 \rho_{\mathrm{w}} \mathrm{g} c_{H}\left(\frac{\bar{\alpha}}{\bar{\alpha}_{\mathrm{PM}}}\right)^{2} a^{2} \bar{\omega} .
$$

In this chapter, we derived four spectral estimates of the dissipation based on the wave evolution models. To evaluate the total dissipation based on these models, we need measurements of both the wave spectrum and the wind speed. In the next chapter, we will describe an experiment whose measurements will be used to compute the surface wave energy dissipation using these models. In Chapter 5, we will then examine the spectral characteristics of these estimates and compare these results with ambient noise measurements. 


\section{EXPERIMENT}

In the literature review, we saw that although there have been numerous studies that clearly show that there is a well-correlated relationship between wind speed and the prevailing underwater ambient noise in the Knudsen range, the mechanics behind this relationship is not well understood. It is believed that the sound generated by breaking waves are responsible for the wind dependent noise. We pointed out that although laboratory experiments have shown that wave field parameters that characterize wave breaking correlate with the acoustic energy radiated, there have been no measurements that show that this relationship is true in the field as well.

The main objectives of our experiment are: to correlate the prevailing noise spectrum level with wave field parameters, particularly wave height, wave slope and wave spectra; to relate these results to measurements of the wind speed dependence of ambient noise; to examine the spectral characteristics of surface wave dissipation models and to study how dissipation correlates with ambient noise.

\subsection{NOBS Description.}

The main thrust of the NOBS (Noise on Basalts and Sediments) experiment was to study the influence of the surface wave spectrum on the very low frequency [O $(1 \mathrm{~Hz})]$ ambient noise on the sea floor. The principal investigator was John Hildebrand of the Scripps Institution of Oceanography (SIO) of the University of California San Diego. The Upper Ocean Physics Group of Robert Pinkel (SIO) and Jerome Smith (SIO) conducted meteorological measurements, as well as measurements of the surface wave directional spectra using an acoustic Doppler sonar system (Smith, 1989) and a wire wave gauge array. 
Table 3.1. Instruments used in this experiment.

\begin{tabular}{|l|c|c|c|}
\hline Instrument & Institution & $\begin{array}{c}\text { Sample } \\
\text { rate (Hz) }\end{array}$ & Type \\
\hline $\begin{array}{l}\text { Directional } \\
\text { hydrophone }\end{array}$ & MIT & 51,200 & $\begin{array}{c}\text { Fabricated at MIT } \\
\text { using ITC-8181A }\end{array}$ \\
\hline Anemometer & SIO & 8 & $\begin{array}{c}\text { Weathertronics } \\
\text { Model 2020/2030 }\end{array}$ \\
\hline Air temperature & SIO & 8 & $\begin{array}{c}\text { Weathertronics } \\
\text { Model 4470 }\end{array}$ \\
\hline Barometer & SIO & 8 & $\begin{array}{c}\text { Weathertronics } \\
\text { Model 7105-A }\end{array}$ \\
\hline Accelerometer & SIO & 8 & Fabricated at SIO \\
\hline Heading & Flip & 8 & Flip instrumentation \\
\hline $\begin{array}{l}\text { Capacitance wire } \\
\text { wavegauge }\end{array}$ & MIT & 8 & Fabricated at MIT \\
\hline $\begin{array}{l}\text { 4-wire resistance } \\
\text { wavegauge array }\end{array}$ & SIO & 8 & Fabricated at SIO \\
\hline Video camera & MIT & N.A. & \\
\hline
\end{tabular}

Although our experiment was unrelated to the main objective of NOBS, observations of the wave field and the meteorological parameters were common to both the ocean bottom studies of Hildebrand and to ours. Table 3.1 summarizes the major instruments used in our study.

The research platform Flip, a manned spar buoy, was towed from San Diego, California and moored in 3000 meters of water approximately $130 \mathrm{~km}$ off the coast of 


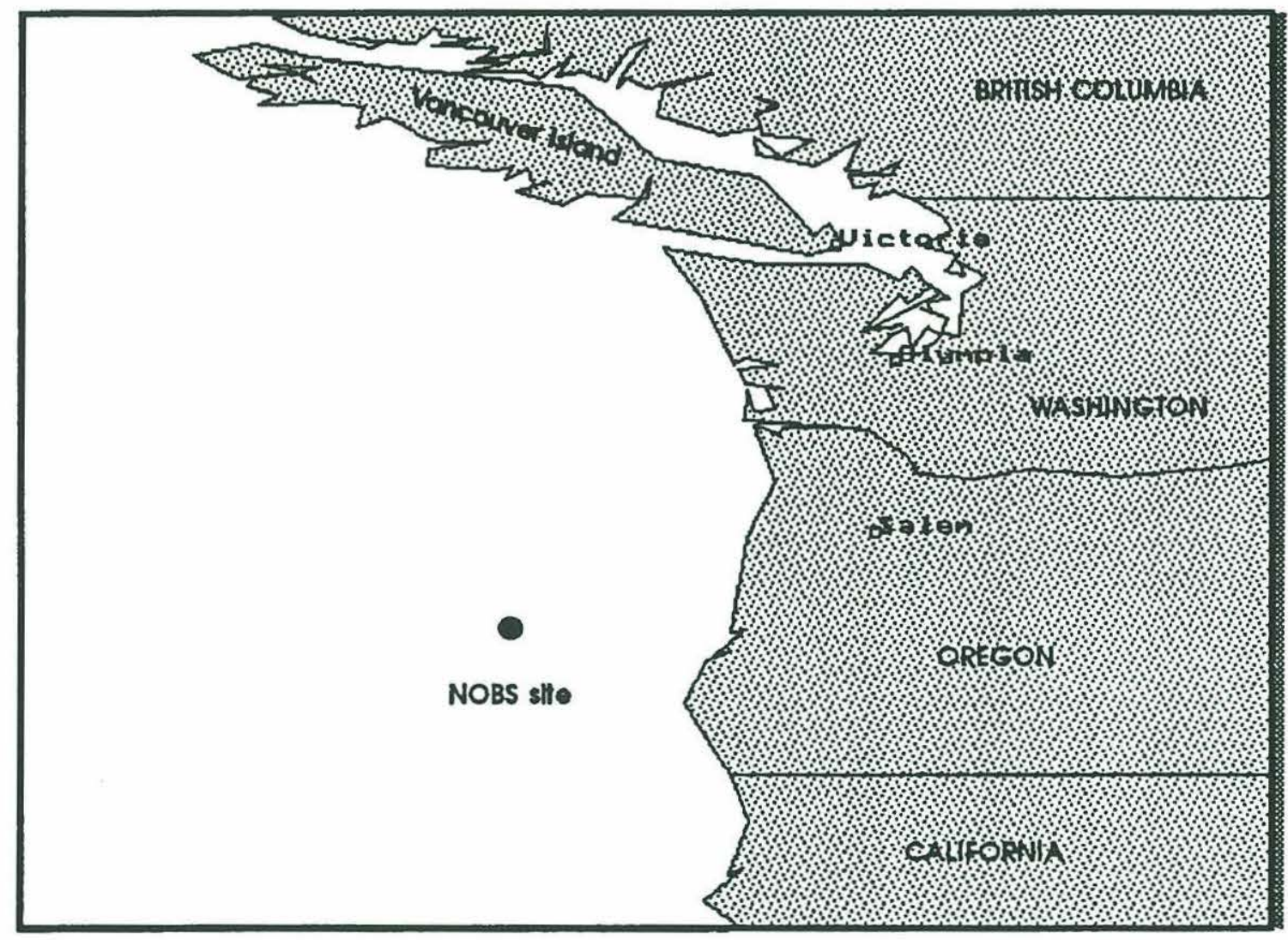

Figure 3.1. A map showing the location of Flip during the NOBS experiment $\left(43^{\circ} 42^{\prime}\right.$ $\left.\mathrm{N}, 125^{\circ} 59^{\prime} \mathrm{W}\right)$. The site is approximately $130 \mathrm{~km}$ west of Oregon and $600 \mathrm{~km}$ south of Vancouver Island. 


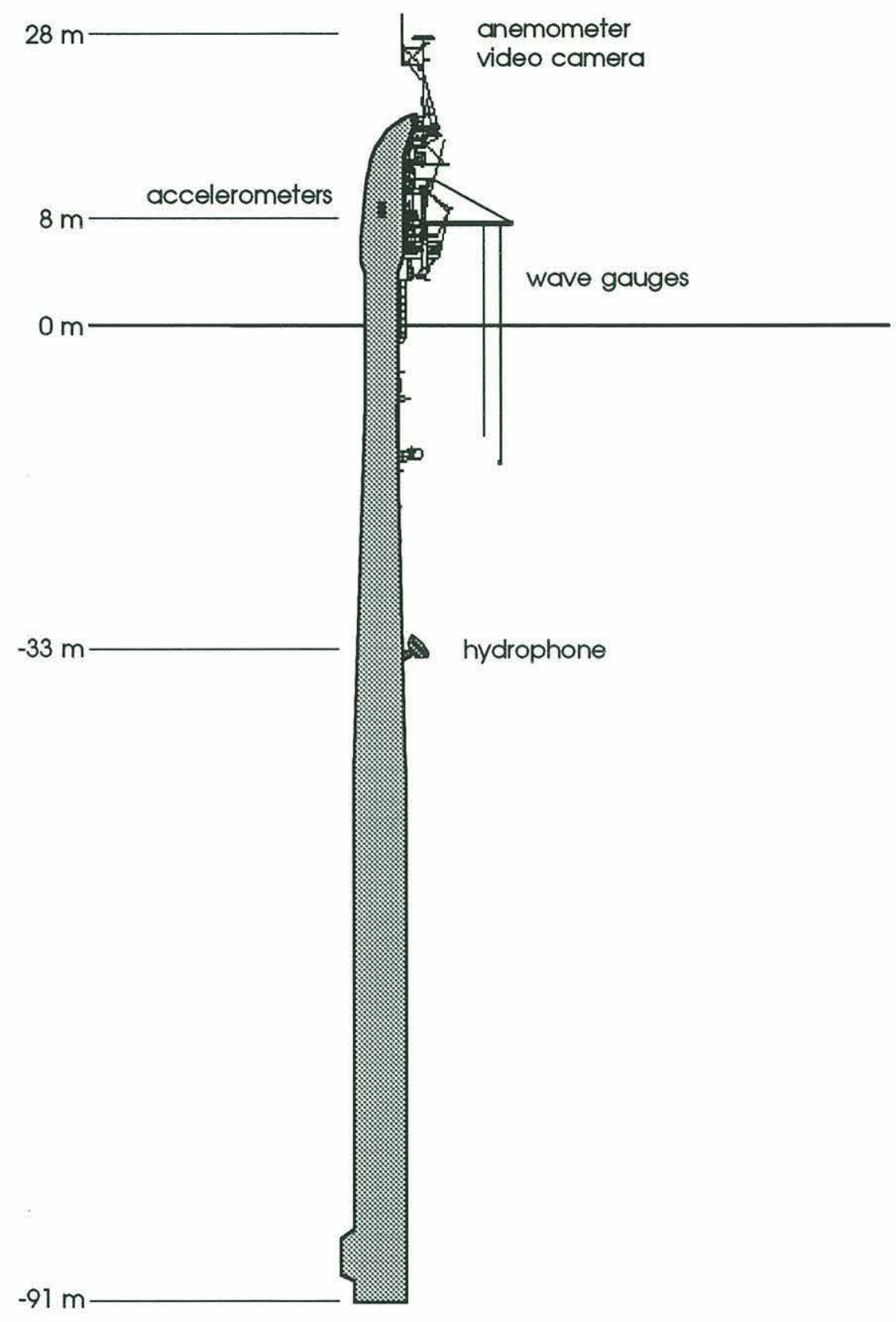

Figure 3.2. A schematic diagram of Flip showing the locations of the different instruments used in the experiment. The capacitance wire wave gauge is 15 meters and the resistance wave gauge array is 17 meters from the hull of Flip. 
Mac Iflx

\begin{tabular}{|c|c|c|}
\hline anemometer & & \\
\hline air temperature & & 오믄 \\
\hline barometer & \multirow[b]{2}{*}{$4 \mathrm{H}$ LP filter } & हీ \\
\hline heading & & \\
\hline accelerometer & $4 \mathrm{~Hz}$ LP filter & \\
\hline capacitance & $4 \mathrm{~Hz} L \mathrm{~L}$ filter & 之岂 \\
\hline resistance wave & \multirow{2}{*}{4 Hz LP filter } & \\
\hline gauge array & & \\
\hline
\end{tabular}

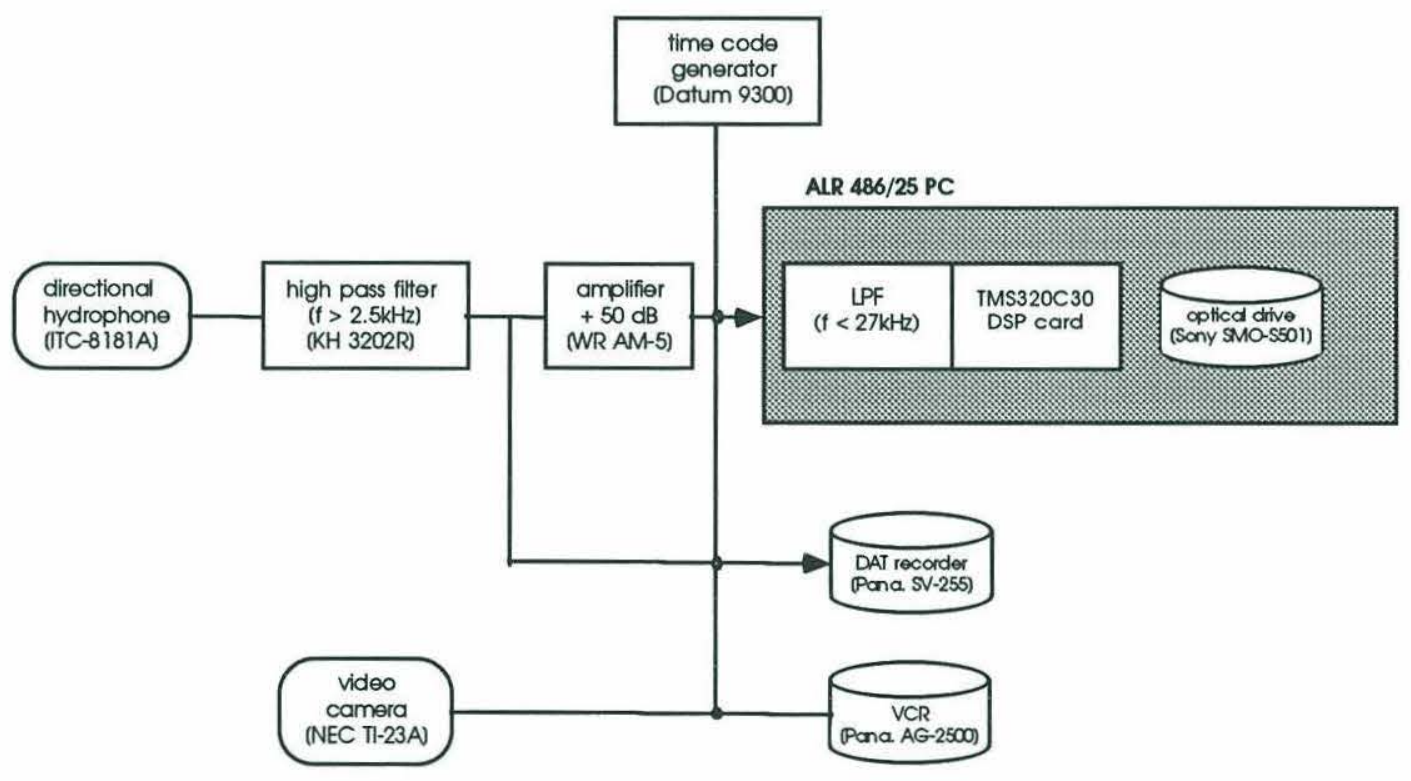

Figure 3.3. Schematic diagram of the sampling and preprocessing method used in gathering and storing data. 
Reedsport, Oregon in September, 1991 (Figure 3.1). Flip was deployed in a 3-pt mooring configuration. The length from the bow to the stern of Flip is approximately 110 meters and the cylindrical hull has a maximum diameter of 6 meters. At vertical attitude, 90 meters of Flip is submerged under water. The section of the hull which intersects the mean water line is approximately 3.8 meters in diameter (Bronson \& Glosten, 1985). The surface waves reflected from the hull of the platform could be observed by eye but these did not appear to have a noticeable effect on the wave field near the capacitance and resistance wavegauges 15 and $17 \mathrm{~m}$ away. Figure 3.2 shows a diagram of Flip and the locations of the different instruments used. A schematic diagram of the data sampling and preprocessing method is shown in Figure 3.3. This is described in detail in the subsequent sections.

\subsection{Directional hydrophone.}

The hydrophone was mounted on Flip 33 meters below sea level. Since one of the initial objectives of this experiment was to track breaking waves using the beam pattern of a directional hydrophone, and because there was some concern that wave splashing on the hull of Flip might dominate the acoustic signal, it was considered desirable to introduce directionality to the hydrophone. This was accomplished by mounting an ITC-8181A omnidirectional hydrophone on the focus of a 42 -inch

parabolic dish (Figures 3.4, 3.5 and 3.6). The parabola was filled with foam to provide a perfectly reflecting surface and improve the directional characteristics of the hydrophone. The resulting beam pattern was obtained from a calibration of the directional hydrophone at the Naval Ocean Systems Center (NOSC) Transducer Calibration Facility in San Diego, CA. The results of the NOSC calibration are given in Appendix A.1. 


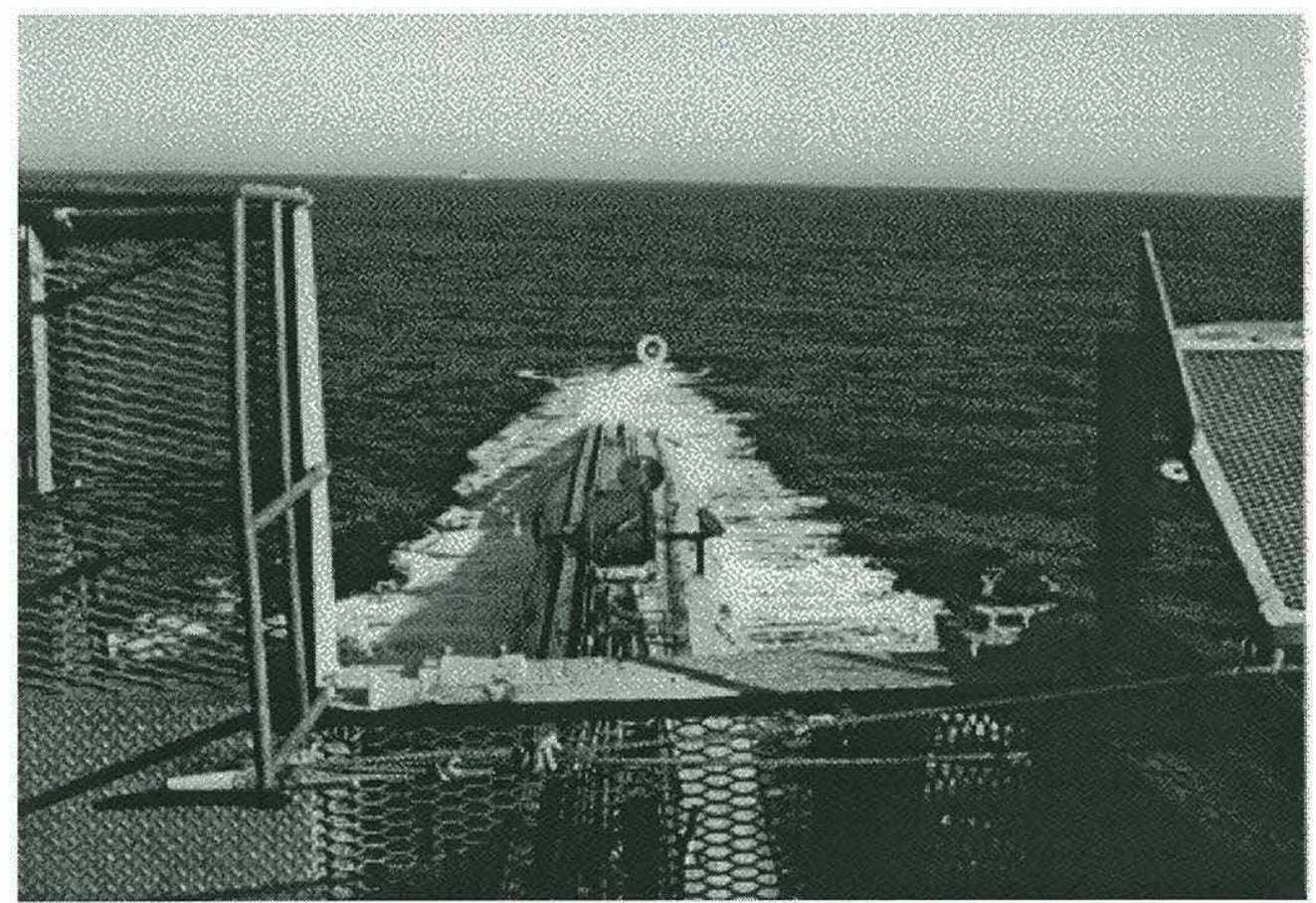

Figure 3.4. A photograph of the directional hydrophone taken immediately after the platform 'flipped' from vertical to horizontal attitude. The photograph was taken from a location near the bow of Flip. The hydrophone is the dark circular disk at the center of the photograph. 


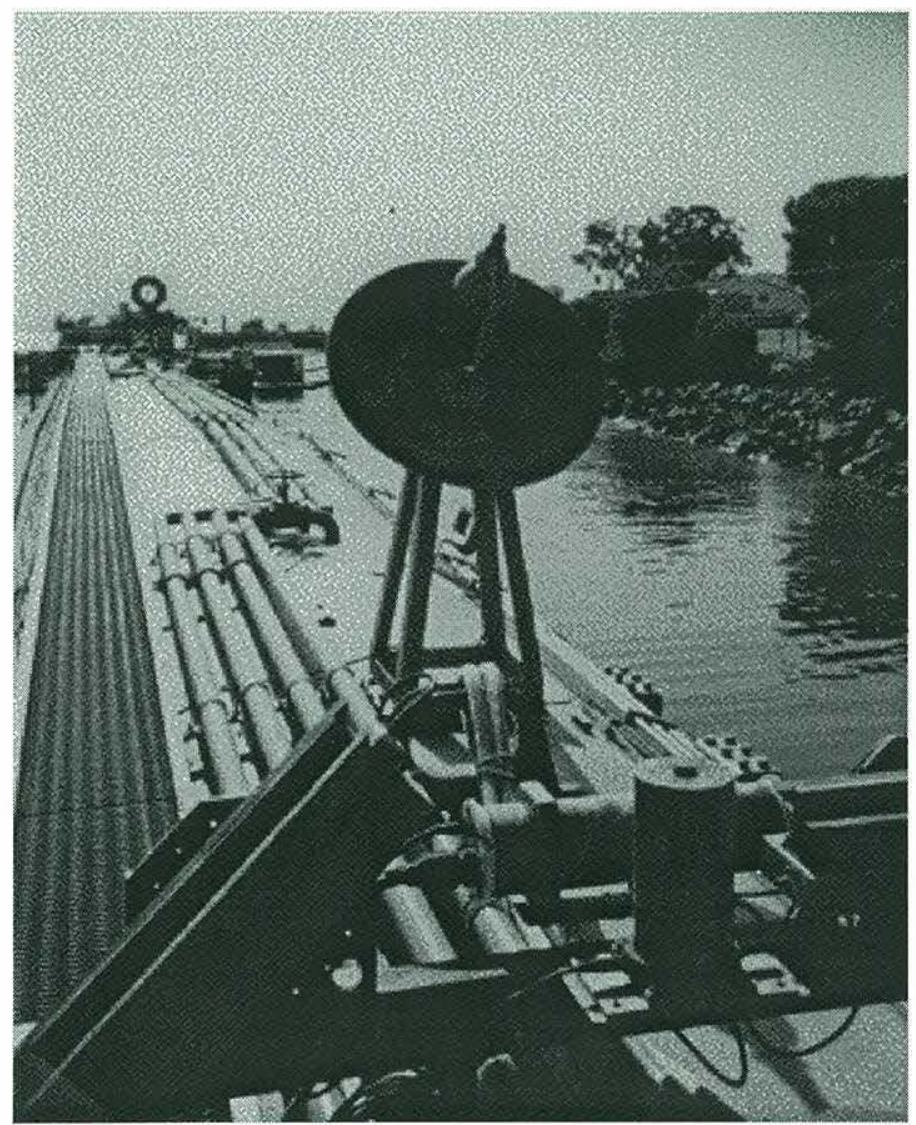

Figure 3.5. A close-up photograph of the directional hydrophone while Flip was in port in San Diego. The instruments in the foreground are the SIO Doppler sonars used to image the sea surface. 


\section{Chapter 3}

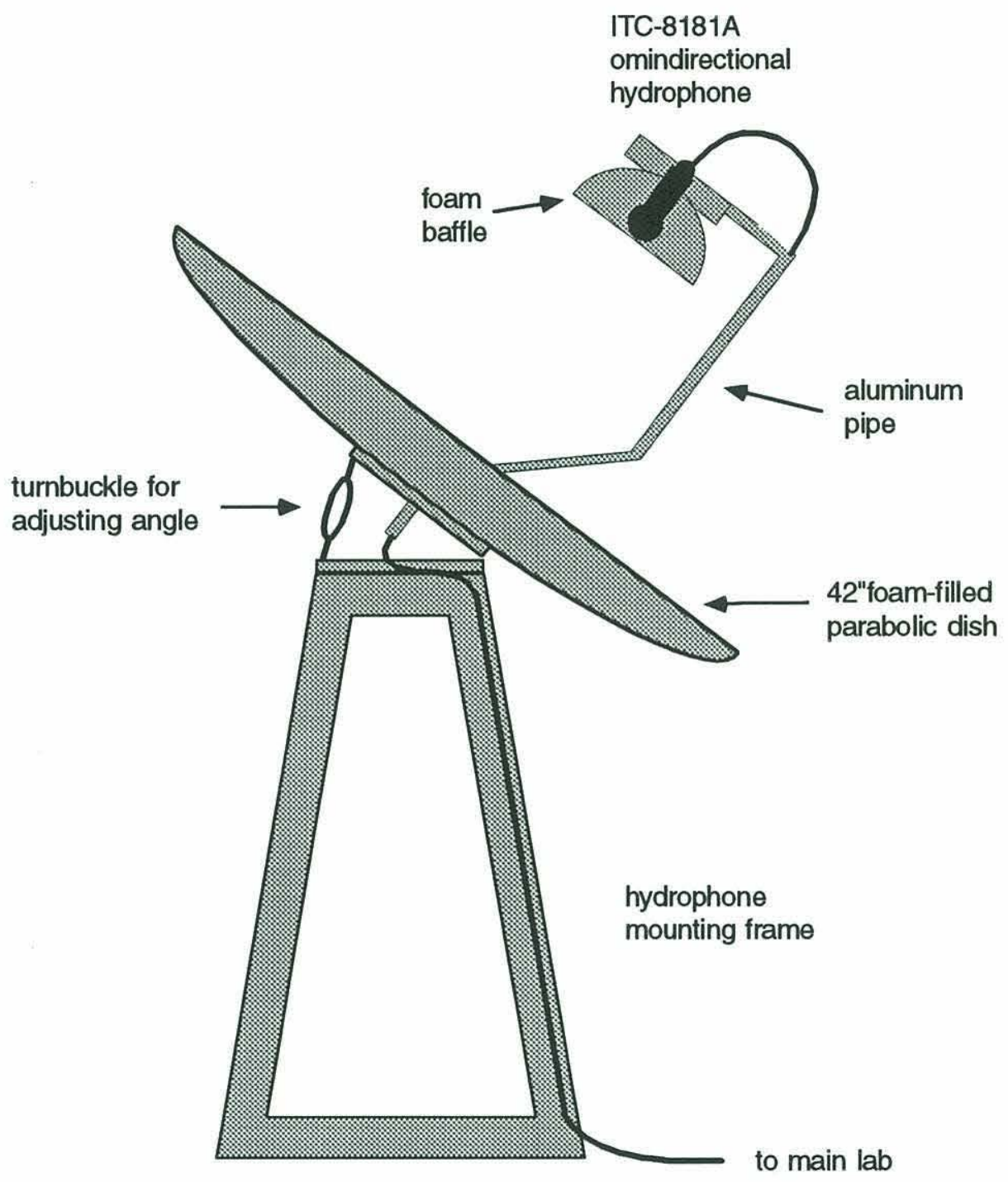

Figure 3.6. A simplified diagram of the directional hydrophone and the mounting. The dimensions are not to scale. 
The configuration resulted in a 3- $\mathrm{dB}$ main lobe with a $25^{\circ}$ included angle at $3 \mathrm{kHz}$ and $6^{\circ}$ at $15 \mathrm{kHz}$. The centerline of the parabola was oriented upward at a $45^{\circ}$ angle from the longitudinal axis of Flip. At $3 \mathrm{kHz}$, the main lobe creates an elliptical footprint on the sea surface whose major and minor axes are 30 meters and 15 meters long. At 25 $\mathrm{kHz}$, the major and minor axes of the elliptical footprint are 3.5 meters and 7 meters long.

A video camera mounted on the crow's nest $28 \mathrm{~m}$ above sea level was oriented towards the footprint of the hydrophone main lobe. At that location, the camera can view a $5.2 \mathrm{~m} \times 4.8 \mathrm{~m}$ section of the sea surface. Four-hour videos of the ocean surface were taken every day starting at 2 PM Pacific Daylight Time (PDT) or 2100 UT. The video image was embedded with the time code to synchronize the video data with the acoustic record. The video data was then recorded using a Panasonic AG-2500 video cassette recorder.

\subsubsection{Data sampling and preprocessing}

Figure 3.3 shows a schematic diagram of the hydrophone data conditioning and sampling method used. The hydrophone signal was high passed at $2.5 \mathrm{kHz}$ using a Krohn-Hite 3202R filter, amplified 50 dB using a Wilcoxon Research AM-5 Low Noise Amplifier, and low-pass filtered at $27 \mathrm{kHz}$, sampled \& preprocessed using the procedure described in the next paragraph using an ALR 486/25 PC equipped with a Spectrum Signal Processing TMS320C30 Real Time Board. This add-in board is an independent computer that has a specialized digital signal processing unit. It communicates with the host PC through the 16-bit ISA bus. The data was stored on 650 MB Sony EDM-1DA1 magneto-optical disks. Selected ambient noise data were recorded in DAT cassettes using a Panasonic SV-255 DAT recorder. 
Prior to the start of each 10-minute sampling segment, the time code generated by a Datum 9300 Time Code Generator was sampled from the PC serial port and recorded. The hydrophone data was then sampled at $51.2 \mathrm{kHz}$ using the TMS320C30 Real Time Board. The power spectrum was computed and averaged from nine 512-point FFTs and then sent to the RAM of the host PC every 0.1 seconds (approximately). This method generated 6000 256-point real power spectra during each 10-minute sampling segment which were stored in 2-byte integer records. The time code was sampled and recorded at the end of the segment prior to storing the data in the optical drive.

\subsubsection{Data processing.}

The ambient noise spectrum level $\mathrm{N}(\mathrm{f})\left[\mathrm{dB}\right.$ re $\left.1 \mu \mathrm{Pa}^{2} / 1 \mathrm{~Hz}\right]$ for an equivalent omnidirectional hydrophone was computed from the raw power spectrum R(f) using the formula

$$
N(f)=R(f)-S A+S L(f)+A G(f)
$$

where $R$ is in $\mathrm{dB}$ re 1 Volt ${ }^{2} / 1 \mathrm{~Hz}, \mathrm{SA}$ is the signal amplification (50 dB), and SL is the hydrophone sensitivity level in $\mathrm{dB}$ re $1 \mu \mathrm{Pa}^{2} / 1 \mathrm{Volt}^{2}$ (Figure 3.7). The array gain AG in $\mathrm{dB}$ was computed using the formula (Dyer, 1989)

$$
\mathrm{AG} \equiv 10 \log \frac{\int_{\Omega} \mathrm{Sd} \Omega}{\int_{\Omega} \mathrm{SB}^{2}(\Omega) \mathrm{d} \Omega}
$$




\section{Chapter 3}

where $\Omega$ is the solid angle. In computing AG, radial symmetry was assumed in both the hydrophone pattern B and the sound source pattern S. A compilation of field measurements of the vertical directionality of ambient noise (Urick, 1986) suggest that the sound source pattern can be modeled as $S(\phi)=\cos ^{2} \phi$ where $\phi$ is the angle from the vertical. The details on how AG was computed is given in Appendix A.1. Figure 3.8 shows a plot of AG computed from the beam pattern of the hydrophone.

The interaction between the hydrophone and several of the instruments on Flip led to the contamination of some of the hydrophone signal. The main noise sources were characterized as follows:

1. Unidentified noise sources covering a broad band of frequencies above $14 \mathrm{kHz}$ made acoustic information in this portion of the spectrum inaccessible in a consistent manner. The acoustic data above $14.2 \mathrm{kHz}$ was considered to be unusable.

2. Flip operated an acoustic beacon which transmitted a 12 -second $4 \mathrm{kHz}$ frequency tone every 2 min 40 seconds. Higher order harmonics at 8 and $12 \mathrm{kHz}$ of this tone were also sampled by the hydrophone. The data was corrected by neglecting the power spectra from the acoustic data that were sampled while the sonar beacon was in operation.

3. The acoustic Doppler sonar mounted on the hull of Flip generated 10millisecond noise pulses every 0.625 seconds. While the pulses were at $195 \mathrm{kHz}$, they produced broad band electrical noise which contaminated acoustic frequencies well within our hydrophones range. This has resulted in the elimination of approximately one-fifth of all acoustic power spectra. 


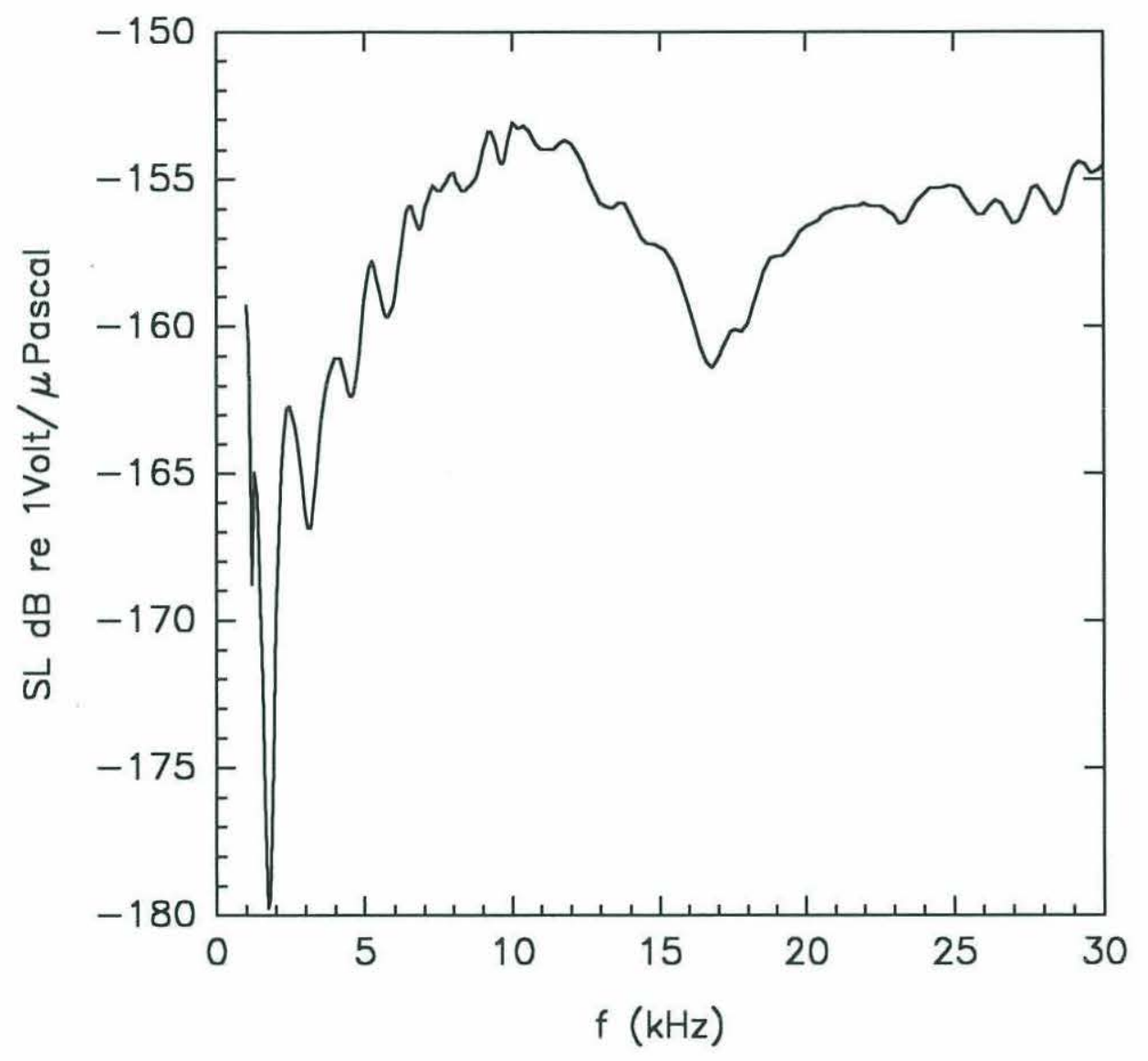

Figure 3.7. Sensitivity level (SL) of the directional hydrophone in $\mathrm{dB}$ re $1 \mathrm{Volt}^{2} / 1 \mu \mathrm{Pa}^{2}$ obtained from a calibration of the instrument at the Naval Ocean Systems Center. 


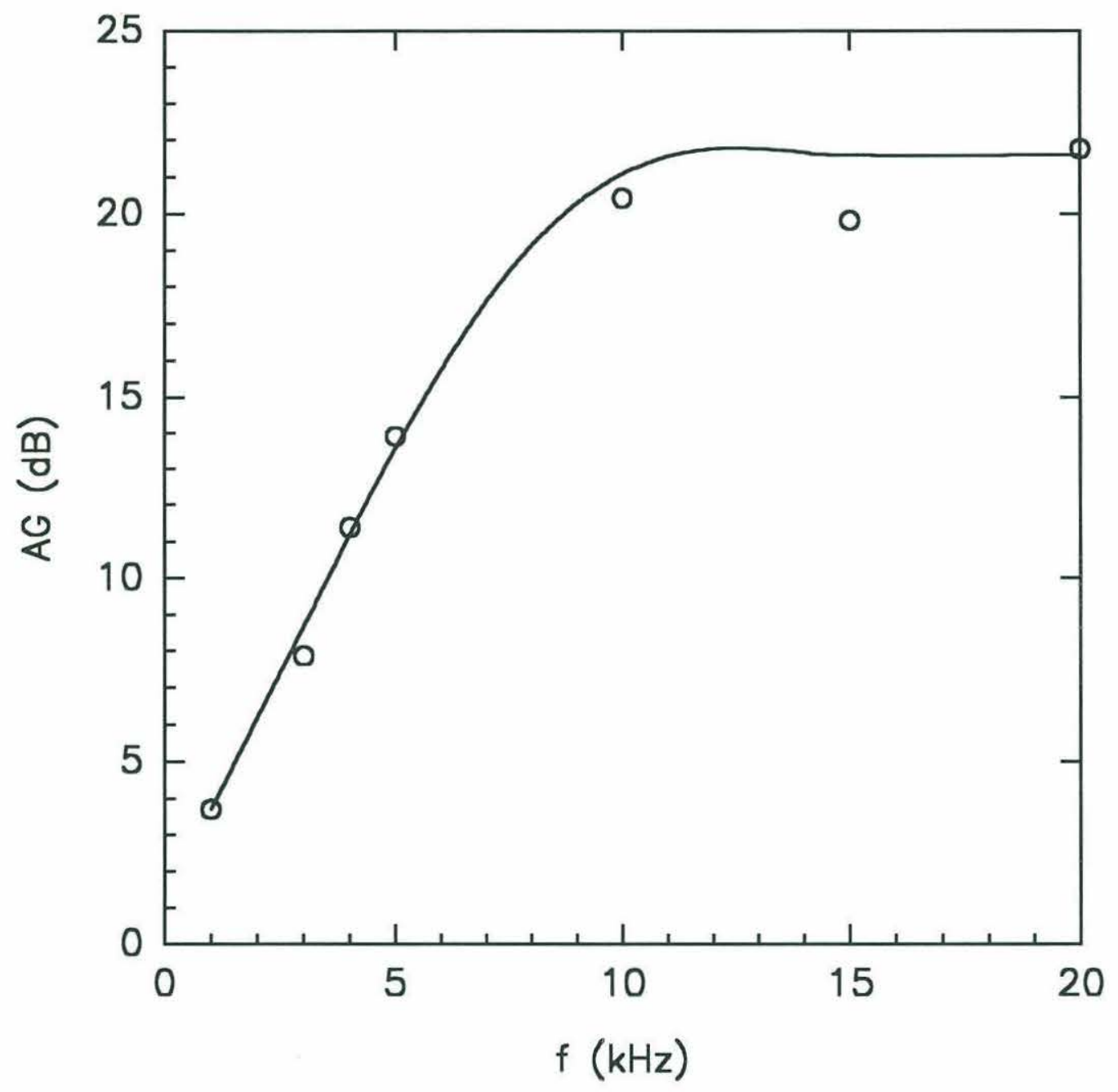

Figure 3.8. Plot of array gain (AG) of the directional hydrophone as a function of frequency. AG values computed from NOSC calibration are denoted by $\mathrm{O}$. 
Figure 3.9 shows some typical unsmoothed power spectra of the ambient noise [dB re $\left.1 \mu \mathrm{Pa}^{2} / 1 \mathrm{~Hz}\right]$ for wind speeds between 4-9 meters/second. The spectra were averaged over one hour. The broad peak at $2.5 \mathrm{kHz}$ is due to the high pass filter cutoff at that frequency.

\subsection{Environmental instruments.}

The data from environmental sensors, the wave gauges, the compass and the accelerometers were sampled at $8 \mathrm{~Hz}$ by a Macintosh IIfx equipped with an NB-MIO16 analog-digital converter (National Instruments, Austin, TX) [see Figure 3.3]. The data, which were stored on optical disks, were provided to us by Jerome Smith of SIO.

The wind speed U was measured by a Weathertronics (Qualimetrics, Sacramento, CA) Model 2020 3-cup anemometer while the wind direction was measured by a Model 2030 vane. Both instruments were mounted on a crossbar positioned at $28 \mathrm{~m}$ above sea level. These measurements were verified during the experiment by comparing them with readings from the Flip anemometer three times per day. One hour averages of $U$ were later computed and reduced to the wind speed at 10 meters

elevation $\mathrm{U}_{10}$. In computing $\mathrm{U}_{10}$, we used the implicit wave age dependent method for computing the friction velocity $\mathrm{u}_{*}$ and the wind speed profile $\mathrm{U}_{\mathrm{z}}$ described by Maat, Kraan \& Oost (1991). Details are given in Section 4.1.1.

Air temperature measurements were sampled using a Weathertronics Model 4470 Platinum Resistance Sensor. The barometric pressure was measured using a Weathertronics Model 7105-A analog barometer. Although instruments for measuring the water temperature and relative humidity were provided, these devices failed to function properly. 


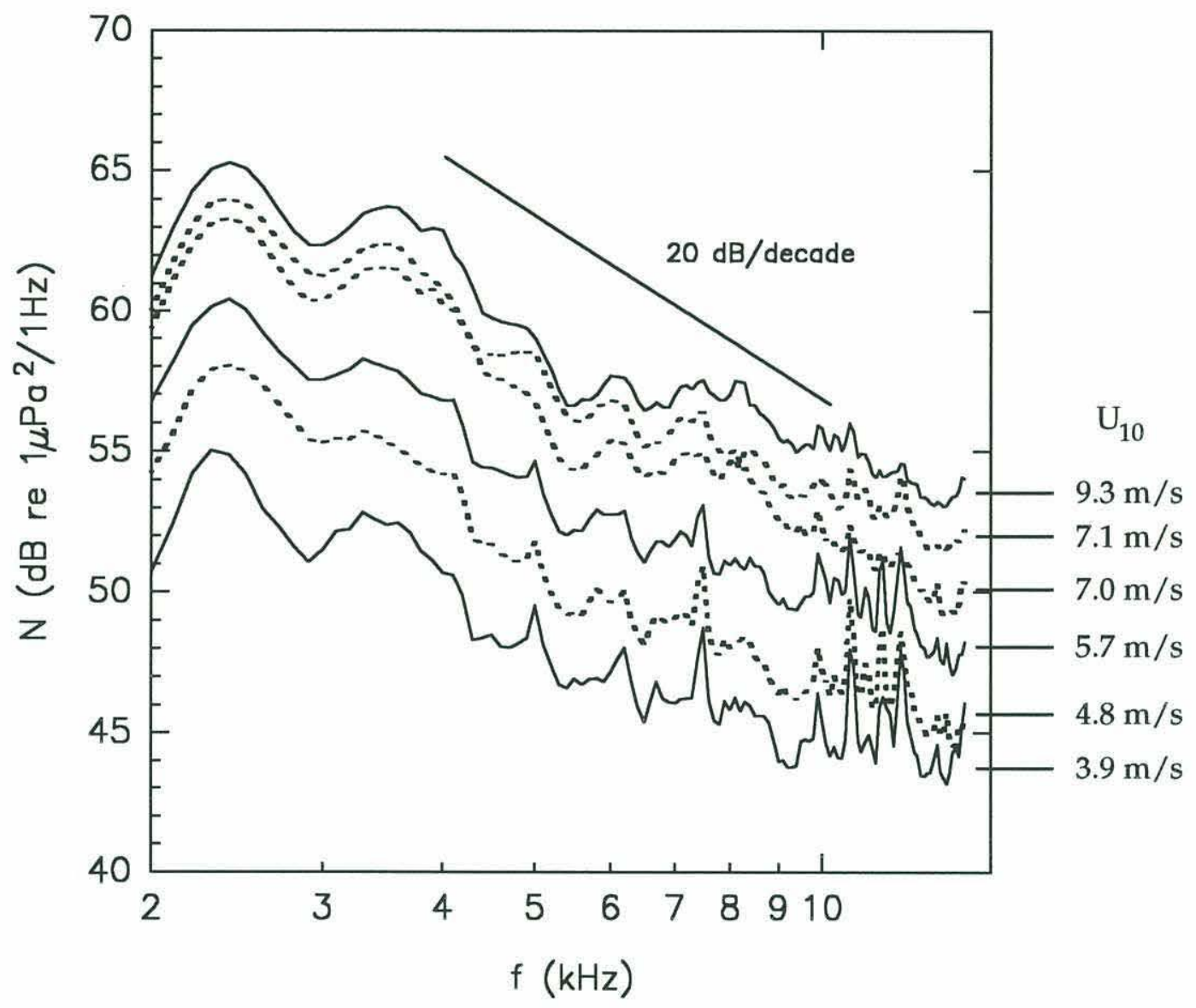

Figure 3.9. Some typical ambient noise power spectra in $\mathrm{dB}$ re $1 \mu \mathrm{Pa}^{2} / 1 \mathrm{~Hz}$ at various wind speeds. 


\subsection{Wave gauges, accelerometers and compass.}

Two independent measurements of the surface waves were used. An array consisting of four Nichrome resistance wire wavegauges designed and built at the Scripps Institution of Oceanography (SIO) was deployed on a boom $8 \mathrm{~m}$ above the mean sea level and 17 meters from the hull. The wires were suspended from the ends of a cross such that the wires are $0.5 \mathrm{~m}$ from the center. The ground wire was suspended from the center of the cross. A spreader was placed at the lower end of the wires to keep them apart. The wave slope and directional wave spectra calculations presented in this study use this data. Figure 3.10 shows a photograph of the 4-wire wave gauge array mounted on the boom taken from the deck of the electronics laboratory of Flip. Figure 3.11 shows the spreader as it is being lowered prior to deploying the wave gauge array at the end of the boom.

A capacitance-type Tantalum wire wavegauge built at MIT based on a design developed at the Applied Physics Laboratory, Johns Hopkins University was also deployed along the boom 15 meters from the hull of Flip. It was calibrated in situ four times during the 12-day observation period. The field calibration of the capacitance wavegauge was accomplished by raising or lowering the wire at 0.5 meter intervals, sampling the voltage for 50 seconds, and computing the mean voltage at each position. The resulting elevation vs. voltage calibration curve was computed using a linear least squares fit of the sample points (Figure 3.12).

An IMET Positional Sensor Package located in the working laboratory recorded the apparent acceleration due to the motion of Flip. It was developed by the Upper Ocean Physics Group at SIO and it measures acceleration and tilt components in the 
Chapter 3

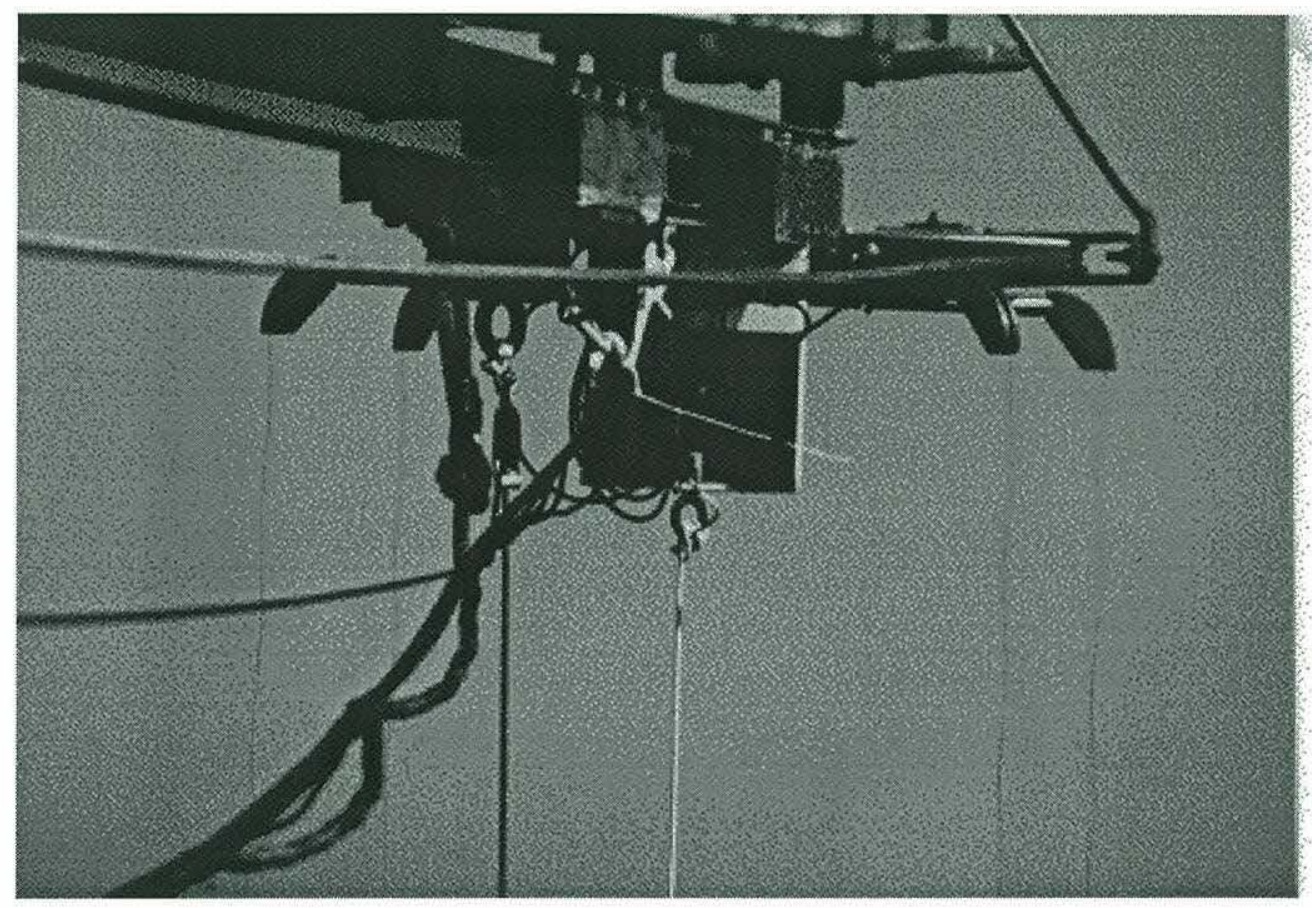

Figure 3.10. Photograph of the 4-wire resistance wave gauge array and the capacitance wire wave gauge (heavy black cable) deployed at the end of the boom. The heavy white cable near the center of the photograph is the ground wire. 


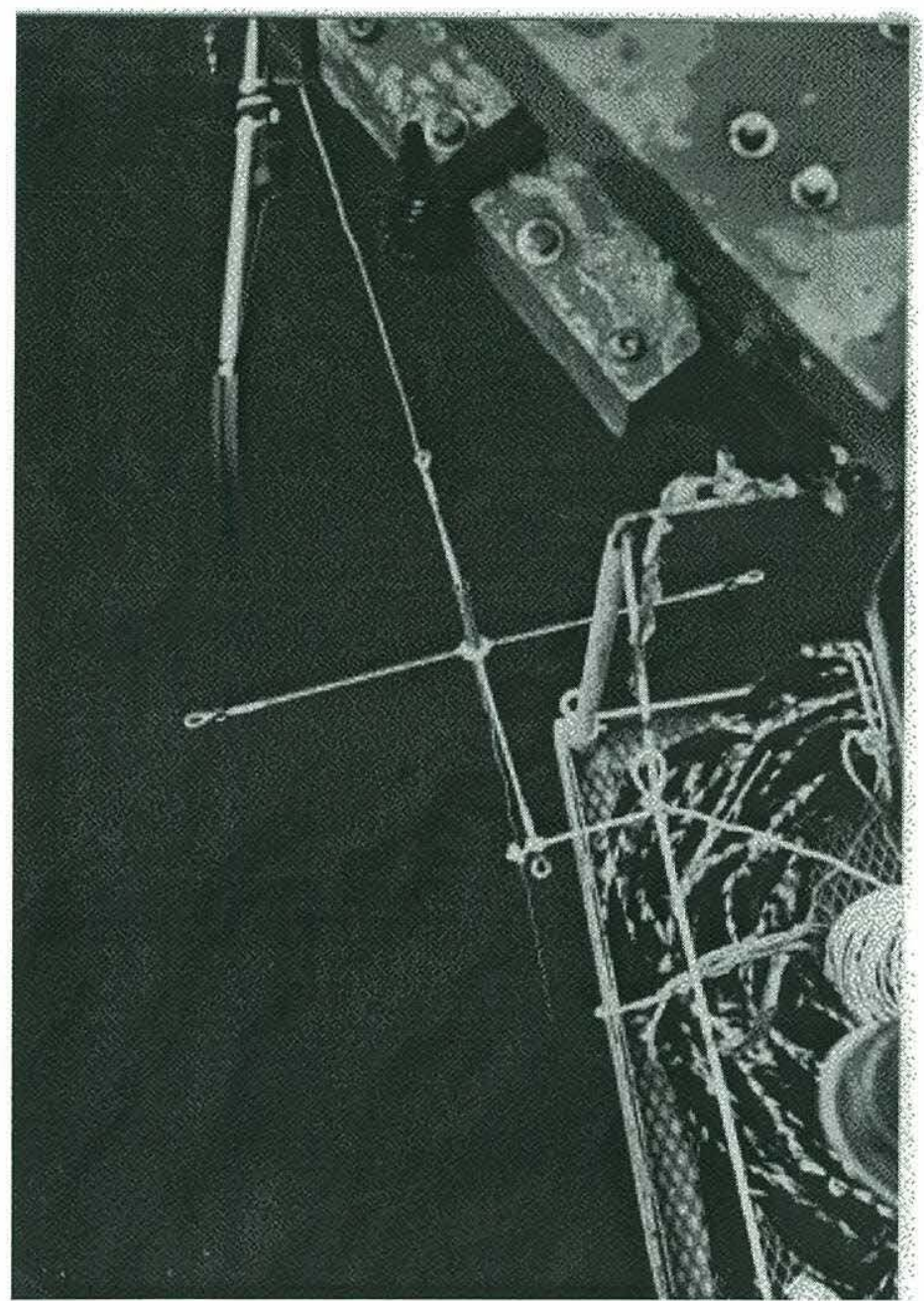

Figure 3.11. Photograph of the bottom spreader bar being lowered to position prior to full deployment of the wire wave gauge array. The bar is made of stainless steel and each arm is 0.5 meters long. 


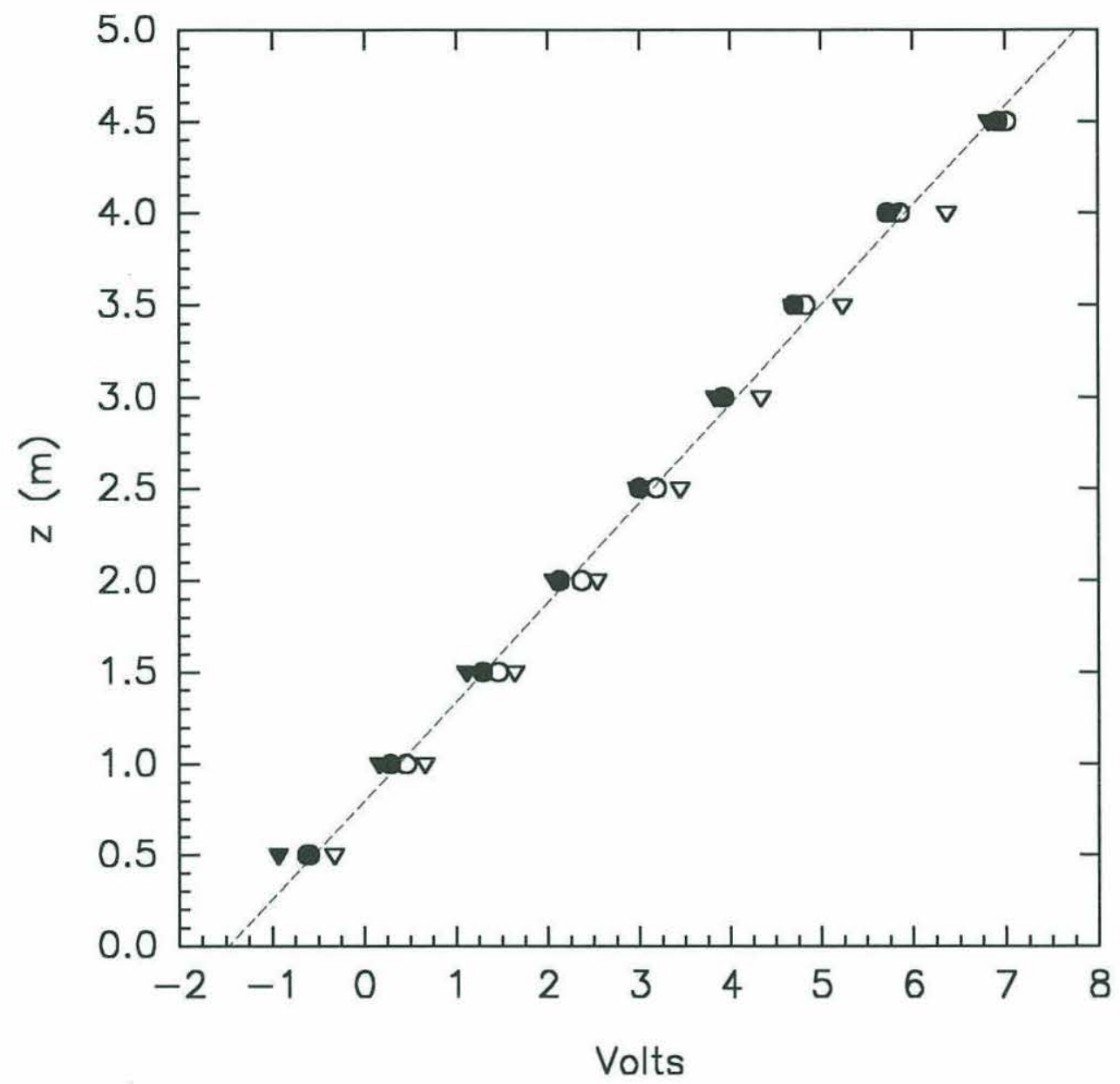

Figure 3.12. Plot showing the values for the four different field calibrations of the capacitance wire wave gauge. The dashed line is a linear least square fit to the sample points. The equation for the best linear least squares fit is

$$
\mathrm{a}_{\mathrm{c}}(\mathrm{m})=0.541 \mathrm{v}_{\mathrm{c}}(\text { Volts })+0.800
$$

where $a_{c}$ is the calibration position and $v_{c}$ is the mean voltage reading at that position. 
horizontal and vertical $(x, y, z)$ directions. The signal from the wavegauges, the accelerometers and Flip's compass were low-passed at $4 \mathrm{~Hz}$ and sampled at $8 \mathrm{~Hz}$.

\subsection{Wave spectra.}

The power spectra of the time series of the sea surface elevation a(t) obtained from the capacitance wire wave gauge were used to examine the evolution of the wave field during the experiment. If we assume that the spatial and temporal probability distribution function of the wave height is stationary over a finite time period $\mathrm{T}$, then the finite Fourier transform pair (Bendat \& Piersol, 1986, p. 130)

$$
\begin{aligned}
& A(f)=\int_{0}^{T} a(t) e^{j 2 \pi f t} d t \\
& a(t)=\frac{1}{\pi} \int_{0}^{1 / T} A(f) e^{-j 2 \pi f t} d f
\end{aligned}
$$

can be used to compute the autocorrelation (or power) spectrum of the wave field

$$
\bar{\Phi}(\mathrm{f}, \mathrm{T})=\frac{1}{\mathrm{~T}} \mathrm{~A}(\mathrm{f}, \mathrm{T}) \mathrm{A}^{*}(\mathrm{f}, \mathrm{T})
$$

where $\omega=2 \pi \mathrm{f}=2 \pi / \mathrm{T}$.

We approximated the continuous spectrum with the equivalent discrete power spectrum computed from the discretely sampled data. This was accomplished by subdividing the time series into finite overlapping records of length $N$ with the 


\section{Chapter 3}

overlaps $N / 2$ records long. The $N$-point records were then windowed using an $N$ point Hanning taper

$$
w_{i}=0.5[1-\cos (2 \pi i / N)] \quad i=1, \ldots, N
$$

to reduce sidelobe leakage. Windowing the data introduces a reduction in the total energy in the time series which may be corrected by multiplying the Fourier transform with the square root of the variance of the window. For a Hanning window, this scale factor is $\sqrt{\frac{8}{3}}$ (Bendat \& Piersol, p. 396). The discrete Fourier transform of the windowed time series is therefore given by

$$
\mathrm{A}\left(\mathrm{f}_{n}\right)=\Delta \mathrm{t} \sqrt{\frac{8}{3}} \sum_{i=0}^{N-1} \mathrm{a}_{i} w_{i} \mathrm{e}^{\mathrm{j} 2 \pi i n / N}
$$

where $\mathrm{j}=\sqrt{-1}, \Delta \mathrm{t}$ is the sample time step, and $n=-\frac{N}{2}, \ldots, \frac{N}{2}$. The discrete Fourier transform was computed using the Danielson-Lanczos Fast Fourier transform (FFT) algorithm described in Press et al (1986, p. 390ff). The wave height power spectrum $\Phi(\mathrm{f})$ computed from $\mathrm{A}$ is then given by

$$
\Phi\left(\mathrm{f}_{n}\right)=\frac{1}{N \Delta \mathrm{t}} \mathrm{A}\left(\mathrm{f}_{n}\right) \mathrm{A}^{*}\left(\mathrm{f}_{n}\right)
$$

where the asterisk $\left(^{*}\right)$ denotes complex conjugation.

In this experiment, we used a value of $N=1024$. For a sampling interval $\Delta t=0.125 \mathrm{~s}$, this gives a time window $\mathrm{T}=N \Delta \mathrm{t}=128 \mathrm{~s}$. To reduce the variance, the power spectrum 
in Equation 3.7 was computed from an average of $K=59$ power spectra of the wave gauge time series. The 1024-point data segments were overlapped 512 points and the resulting power spectrum represented approximately an hour of the data. The onesided power spectrum which will be used throughout this work is

$$
\Phi\left(\mathrm{f}_{n}\right)=\frac{2}{\mathrm{~K} N \Delta \mathrm{t}} \mathrm{A}\left(\mathrm{f}_{n}\right) \mathrm{A}^{*}\left(\mathrm{f}_{n}\right) \quad n=1, \ldots, \frac{N}{2} .
$$

Figure 3.13 shows the characteristics of a typical wave height power spectrum observed during the experiment. The plot was obtained by computing and averaging 1024-point discrete Fourier transforms on a 1-hour capacitance wave gauge record. Three major peaks are observed. Given the location and fetch lengths at the site, a significant peak due to swell is present along with the wind sea peak. The peak below $0.05 \mathrm{~Hz}$ is due to the 27-sec heave and 48-second pitch-roll resonance of Flip which are unresolved. The presence of these peaks is consistent with previous studies of Flip's response to waves (Rudnick, 1967). While the energy spectrum of Flip's motion due to waves has components of higher frequency, their effect on the measured wave energy greater than $0.05 \mathrm{~Hz}$ is small.

\subsection{Wave slope.}

An estimate of the root mean square (RMS) wave slope $s$ was used to parameterize the steepness of the wave field. Figure 3.14 shows a plan view schematic diagram of the location and orientation of the 4-wire resistance wave gauge array. We defined the East direction to be the true $\mathrm{x}$-axis and the North direction to be the true $\mathrm{y}$-axis. A local coordinate system based on the geometry of Flip was also defined such that the direction towards the keel was the Flip $\mathrm{x}$-axis $\mathrm{x}_{\mathrm{F}}$. The orientation of the Flip $\mathrm{y}$-axis $\mathrm{y}_{\mathrm{F}}$ 

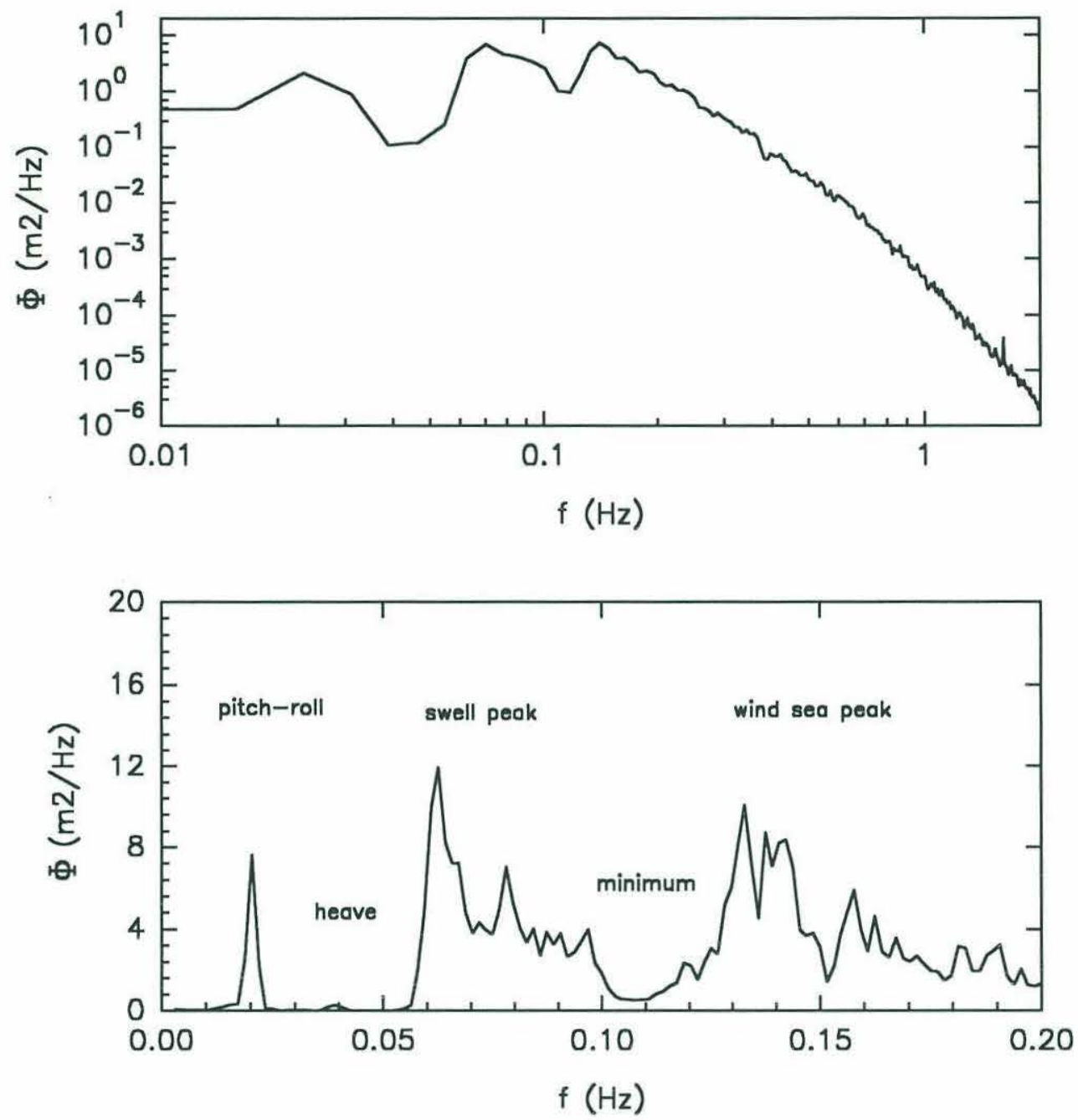

Figure 3.13. Plots showing the characteristics of a typical wave height power spectrum. The data were sampled at $8 \mathrm{~Hz}$. The spectra were computed from a one hour wave gauge record with $U_{10}=8 \mathrm{~m} / \mathrm{s}$. a) Power spectrum computed from 1024point FFTs used in throughout this work. b) Power spectrum over the same period computed from 2560-point FFTs to resolve the lower frequency spectral peaks. 
follows from the right-hand rule. A third coordinate system based on the wire wave gauge array was also defined such that the array $\mathrm{x}$-axis $\mathrm{x}_{\mathrm{w}}$ bisects the angle formed by the arms from which wires 1 and 4 were suspended. This axis is also perpendicular to the boom and the array $\mathrm{y}$-axis $\mathrm{y}_{\mathrm{w}}$ is colinear with the boom.

The orientation of the boom and of the wave gauge array are given in Figure 3.14. The angle between the keel of Flip and the magnetic North $\theta_{\mathrm{F}}$ was measured by a compass on Flip. The signal was sampled at $8 \mathrm{~Hz}$ together with the wire wavegauge signal.

From Figure 3.14, the angle between the true coordinate system and the array coordinate system is

$$
\begin{aligned}
& \theta=\theta_{\mathrm{F}}-\left(119^{\circ}+90^{\circ}\right) \\
& \theta=\theta_{\mathrm{F}}-209^{\circ}
\end{aligned}
$$

where $\theta$ is defined to be positive in the clockwise direction. The instantaneous slope of the sea surface $s(t)=\left(s_{x}, s_{y}\right)$ can be estimated from the simultaneous sea surface elevation $\mathrm{a}_{\mathrm{i}}(\mathrm{t})$ measurements of the four wire wave gauges, $\mathrm{i}=1,2,3,4$. In the local coordinate system $\left(\mathrm{x}_{\mathrm{w}}, \mathrm{y}_{\mathrm{w}}\right)$, the slope is computed from the mean of the finite difference of the wave elevation between two wires

$$
\begin{aligned}
& s_{\Delta x}^{w}(t)=\frac{\left[a_{1}(t)-a_{4}(t)\right]+\left[a_{2}(t)-a_{3}(t)\right]}{2 \Delta x} \\
& s_{\Delta y}^{w}(t)=\frac{\left[a_{2}(t)-a_{1}(t)\right]+\left[a_{3}(t)-a_{4}(t)\right]}{2 \Delta y}
\end{aligned}
$$




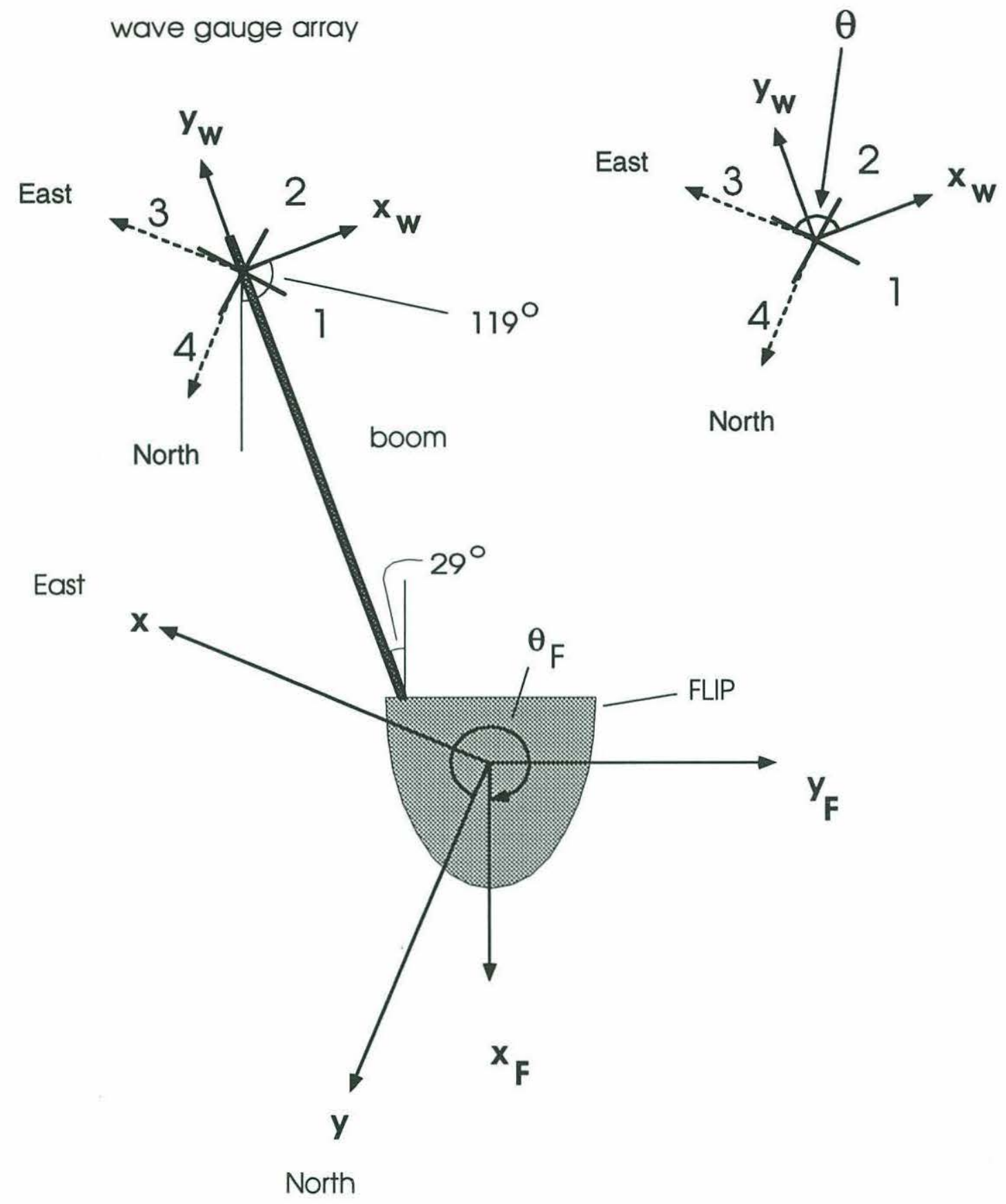

Figure 3.14. Plan view schematic diagram showing the location and orientation of the resistance wave gauge array and the coordinate systems used in computing the wave slope s. 


\section{Chapter 3}

where $\Delta x=\Delta y=0.7071 \mathrm{~m}$. The wave slope in the true coordinate system is therefore

$$
\begin{aligned}
& \mathrm{s}_{\Delta x}(\mathrm{t})=\mathrm{s}_{\Delta \mathrm{x}}^{\mathrm{w}}(\mathrm{t}) \cos \theta(\mathrm{t})-\mathrm{s}_{\Delta \mathrm{y}}^{\mathrm{w}}(\mathrm{t}) \sin \theta(\mathrm{t}) \\
& \mathrm{s}_{\Delta \mathrm{y}}(\mathrm{t})=\mathrm{s}_{\Delta \mathrm{y}}^{\mathrm{w}}(\mathrm{t}) \sin \theta(\mathrm{t})+\mathrm{s}_{\Delta \mathrm{x}}^{\mathrm{w}}(\mathrm{t}) \cos \theta(\mathrm{t})
\end{aligned} .
$$

The one hour variance of the wave slope components, $\sigma^{2}\left(\mathrm{~s}_{\Delta x}\right)$ and $\sigma^{2}\left(\mathrm{~s}_{\Delta y}\right)$, where

$$
\begin{aligned}
\sigma^{2}(z) & =\frac{1}{N} \sum_{i=1}^{N}\left(z_{i}-\bar{z}\right)^{2} \\
\bar{z} & =\frac{1}{N} \sum_{i=1}^{N} z_{i}
\end{aligned}
$$

were computed from Equation 3.11. The RMS wave slope $s$ is therefore

$$
S=\left[\frac{1}{4} \sum_{n=1}^{4} \sigma^{2}\left(s_{\Delta \mathrm{x}}^{(n)}\right)+\frac{1}{4} \sum_{n=1}^{4} \sigma^{2}\left(\mathrm{~s}_{\Delta \mathrm{y}}^{(n)}\right)\right] .
$$

\subsection{Directional wave height spectra.}

\subsubsection{Theory.}

We used the Maximum Likelihood Method in computing directional spectra using the 4-wire wave gauge array. The method was first used by Capon (1969) in computing the wavenumber spectra of seismic waves. Oakley \& Lozow (1977) showed that in a nearly noise free environment, a sparse wave gauge array of at least three wires can 
theoretically resolve the direction of waves whose wavelengths are several orders of magnitude larger than the size of the array. However, the presence of noise in real arrays sets a finite limit to the resolving power of the array. We present a derivation of the Maximum Likelihood technique for obtaining the directional wave height spectrum $\Phi(\omega, \theta)$ from an array of $N$ wire sensors based on an approach described by Jefferys, Wareham, Ramsden \& Platts (1981). This section is essentially a review of their paper.

Let $A_{i}(\omega)$ be the Fourier transform of the wave elevation time series for wave gauge $i$ at position

$$
\mathbf{r}_{1}=r_{1}\left(\cos \theta_{1}, \sin \theta_{1}\right)
$$

where $r_{i}$ and $\theta_{i}$ are the distance and angle from the origin to the sensor $i, i=1, \ldots, N$. The $N \times N$ cross spectral density matrix $\mathbf{C}$ for sensors $\mathrm{i}$ and $\mathrm{j}$ is then given by

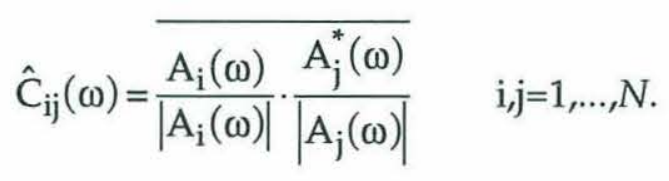

The overbar in the above equation indicates that a sufficient number of Fourier transforms should be averaged to ensure that the cross spectral density estimate $\hat{\mathrm{C}}_{\mathrm{ij}}(\omega)$ is statistically reliable. The asterisk * indicates complex conjugation. It is common to normalize the Fourier transforms by their moduli to avoid errors due to differences in wave gauge calibration. 


\section{Chapter 3}

If the directional wave height spectrum $\Phi(\omega, \theta)$ is expressed as the sum of $M$ plane waves of power $\Phi\left(\omega, \theta_{\mathrm{m}}\right)$ propagating along directions $\theta_{\mathrm{m}}, \mathrm{m}=1, \ldots, \mathrm{M}$, then the true cross power spectral density function is then

$$
\begin{gathered}
\mathbf{C}(\omega)=\sum_{\mathrm{m}=1}^{\mathrm{M}} \boldsymbol{x}\left(\omega, \theta_{\mathrm{m}}\right) \Phi\left(\omega, \theta_{\mathrm{m}}\right) \boldsymbol{x}^{* \mathrm{~T}}\left(\omega, \theta_{\mathrm{m}}\right) . \\
\boldsymbol{x}_{i}\left(\omega, \theta_{m}\right)=\mathrm{e}^{-j \mathbf{k}_{m} \cdot \boldsymbol{r}_{i}} \quad i=1, \ldots, N .
\end{gathered}
$$

The ith component of $x$ is the complex phase lag between the ith wave gauge and the origin for a wave of frequency $\omega$ propagating along the direction $\theta_{\mathrm{m}}$ while $\mathbf{k}$ is the wavenumber vector whose elements are

$$
\mathrm{k}_{\mathrm{m}}=\frac{2 \pi}{\lambda}\left(\cos \theta_{\mathrm{m}}, \sin \theta_{\mathrm{m}}\right)
$$

We implicitly assumed the linear deep water dispersion relation $\omega^{2}=\mathrm{gk}$.

Given the matrix $\hat{\mathbf{C}}(\omega)$ estimated using Equation 3.14, we can invert Equation 3.15 to obtain an estimate of the power spectrum $\hat{\Phi}\left(\omega, \theta_{d}\right)$ of the wave propagating along direction $\theta_{\mathrm{d}}$

$$
\begin{aligned}
\hat{\Phi}\left(\omega, \theta_{\mathrm{d}}\right) & =\mathbf{E}{ }_{\mathrm{d}}^{\mathrm{T}} \mathbf{C}(\omega) \mathbf{E}_{\mathrm{d}} \\
& =\sum_{\mathrm{m}=1}^{\mathrm{M}}\left|\mathbf{E}{ }_{\mathrm{d}}^{\mathrm{T}} \mathbf{z}\left(\omega, \theta_{\mathrm{m}}\right)\right|^{2} \Phi\left(\omega, \theta_{\mathrm{m}}\right) \\
& =\left|\mathbf{E} *_{\mathrm{d}}^{\mathrm{T}} \mathbf{x}\left(\omega, \theta_{\mathrm{d}}\right)\right|^{2} \Phi\left(\omega, \theta_{\mathrm{d}}\right)+\sum_{\mathrm{m}=1 \neq \mathrm{d}}^{\mathrm{M}}\left|\mathbf{E} *{ }_{\mathrm{d}}^{\mathrm{T}} \mathbf{x}\left(\omega, \theta_{\mathrm{m}}\right)\right|^{2} \Phi\left(\omega, \theta_{\mathrm{m}}\right) .
\end{aligned}
$$


To evaluate $\hat{\Phi}\left(\omega, \theta_{d}\right)$, we need to determine the vector $E_{d}$ such that the contribution from $\Phi\left(\omega, \theta_{d}\right)$ is undistorted while the contributions from the other directional components are minimized. We minimize $\Phi\left(\omega, \theta_{\mathrm{d}}\right)$ with respect to $\mathrm{E}_{\mathrm{d}}$ subject to

$$
\left|E_{d}^{* T} \mathbf{x}\left(\omega, \theta_{d}\right)\right|=1
$$

This leads to an estimate of the power in the wave frequency $\omega$ propagating along $\theta_{d}$ (Capon, 1969)

$$
\hat{\Phi}\left(\omega, \theta_{\mathrm{d}}\right)=\left[\mathbf{x}^{* \mathrm{~T}}\left(\omega, \theta_{\mathrm{d}}\right) \hat{\mathbf{c}}^{-1}(\omega) \mathbf{z}\left(\omega, \theta_{\mathrm{d}}\right)\right]^{-1}
$$

The directional spectrum was scaled such that the sum of the wave energy at frequency $\omega$ for all the wave component directions is equal to the spectral energy level of the capacitance wire wave gauge

$$
\Phi(\omega)_{\mathrm{cwg}}=\sum_{\mathrm{m}=1}^{\mathrm{M}} \Phi\left(\omega, \theta_{\mathrm{m}}\right)
$$

Problems regarding the numerical stability of the $\hat{\mathbf{C}}$ matrix can arise when its inverse is computed in Equation 3.18. If there are fewer wave frequencies than wave gauges, then $\hat{\mathbf{C}}$ will be overdetermined and consequently singular. It can be made positive definite and its stability enhanced by introducing a small positive perturbation $R<1$ such that 
wave gauge array

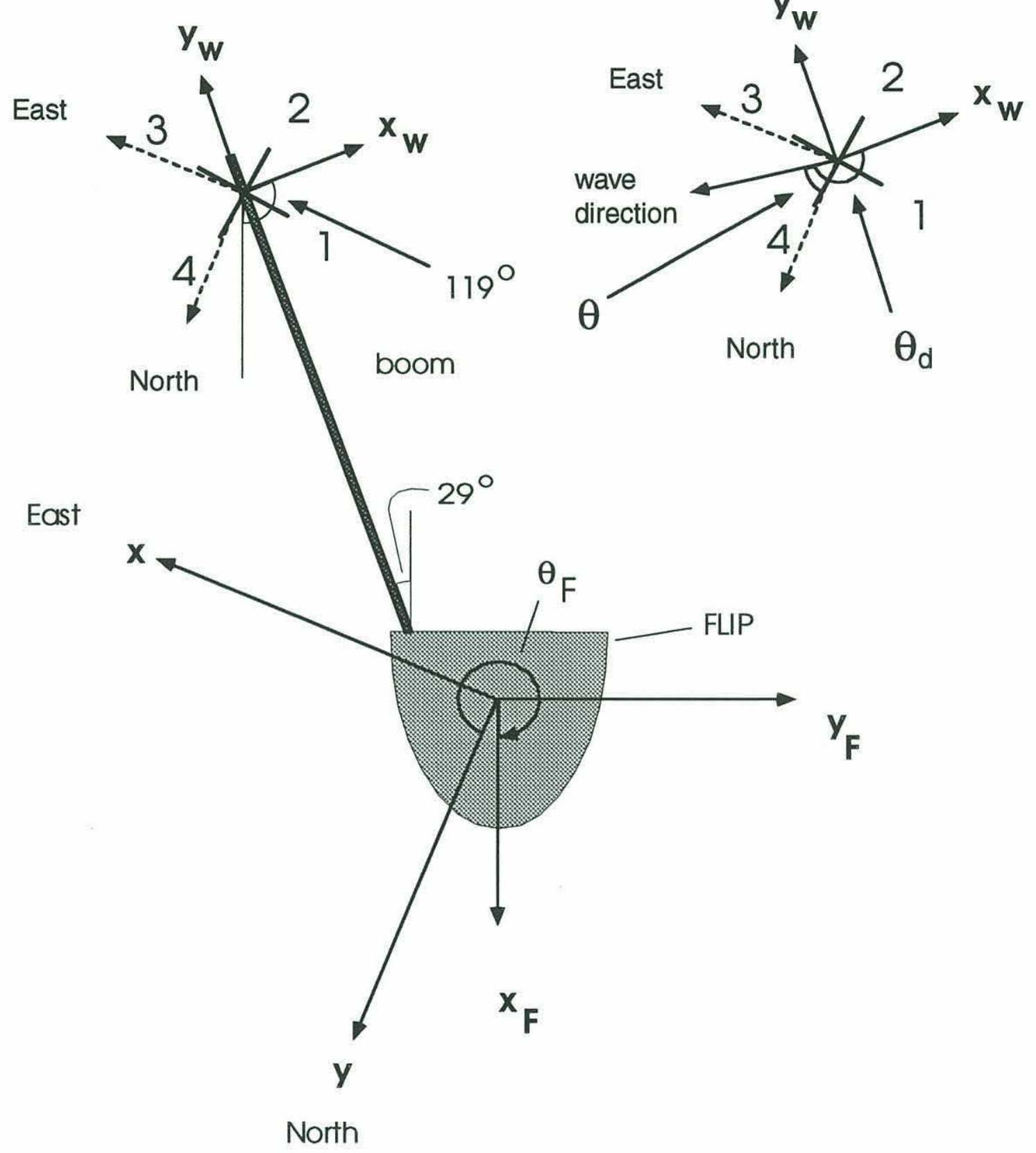

Figure 3.15. Plan view of schematic diagram showing the coordinate system used in computing the directional wave spectrum $\Phi(\omega, \theta)$. 


$$
\hat{\mathbf{C}}^{R}=(1-R) \hat{\mathbf{C}}+R \mathbf{I}
$$

where I is an $\mathrm{NxN}$ identity matrix. This method is equivalent to introducing a small uncorrelated noise whose power is $R$ times the wave energy into the wave gauges.

\subsubsection{Implementation.}

We now outline the basic steps in implementing a practical algorithm for computing the directional spectrum using the maximum likelihood method from the time series of the surface wave elevation measurements $a_{i}(t)$ at wave gauge $i$ for the 4-wire resistance wave gauge array shown in Figure 3.15.

1. Compute the complex phase lags $x_{\mathrm{i}}$ (Equation 3.16) for each wave gauge using the array coordinate system shown in Figure 3.15.

2. Subdivide the time series data $\mathrm{a}_{\mathrm{i}}(\mathrm{t})$ into sample windows of length $N_{w}$. In this experiment, we used $N_{w}=1024$ with a 512-point overlap between sample windows. Since the data was sampled at $8 \mathrm{~Hz}$, each window is 128 seconds long.

3. Window the data to reduce sidelobe leakage. A Hanning window (Equation 3.5) was used.

4. Compute the discrete Fourier transform $A\left(f_{n}\right)$ of the windowed time series using the Fast Fourier Transform method. 
5. Compute the $4 \times 4$ cross spectral density matrix $\hat{\mathbf{C}}(\omega)$ (Equation 3.14). Each $\hat{\mathrm{C}}_{\mathrm{ij}}(\omega)$ estimate was based on 32 minutes of data or 29 spectral averages.

6. Perturb the $\hat{\mathbf{C}}(\omega)$ matrix by introducing a small noise $R$ (Equation 3.20). $R=10^{-6}$ was used.

7. Compute the inverse of $\hat{\mathbf{C}}(\omega)$.

8. Subdivide the $\theta$ space into $\mathrm{M}$ directions. Compute the Maximum Likelihood estimate of the wave spectrum $\Phi\left(\omega, \theta_{d}\right)$ in direction $\theta_{d}$ (Equation 3.18) for all the $M$ directions. We used $M=72$ in this experiment resulting in a $\Delta \theta=5^{\circ}$.

9. Compute the power spectral density of the capacitance wire wave gauge $\Phi(\omega)_{\text {cwg }}$.

10. Rescale the $\Phi$ computed in step 8 so that the sum of all wave directions at a given frequency $\omega$ is equal to $\Phi(\omega)_{\text {cwg }}$ (Equation 3.19).

11. Convert the directional spectrum from the wave gauge array coordinate system to the true coordinate system. From Figure 3.15, the azimuth $\theta$ of a given direction is

$$
\theta=\theta_{d}-119^{\circ}+\theta_{F}
$$

where $\theta_{\mathrm{d}}$ is the angle of that direction from the array $\mathrm{x}$-axis $\mathrm{x}_{\mathrm{W}}$, and $\theta_{\mathrm{F}}$ is the azimuth of Flip's keel. 
Chapter 4

\section{OBSERVATIONS}

\subsection{General conditions}

The observation period of this experiment covered approximately twelve days between September 26, 1991 - October 8, 1991 (Julian day 269 - 281). We began recording the acoustic measurements while the winds blowing from the south were dying down. After one and a half days of relative calm, the wind blew initially from the north (fetch length: $600 \mathrm{~km}$ ) with the wind speed increasing over a day. It stayed in a quasi-steady condition over a period of six days. Although the variability in the wind direction was minimal during the quasi-steady period, the hourly-averaged wind speed U fluctuated between $6-12$ meters/second. The wind then changed direction and blew from the south (fetch length: unlimited) and then died down over the course of a day and a half. The sudden change in wind direction was accompanied by a rapid decline and increase in the wind speed over a period of six hours.

\subsection{Wind speed $U_{10}$ and friction velocity $u_{*}$.}

Although our wind speed measurements were made at 28 meters elevation $\left(U_{28}\right)$, we reduced this value to the wind speed at 10 meters consistent with meteorological convention. It is common to assume a logarithmic wind speed profile near the sea surface of the form (Large \& Pond, 1981)

$$
\mathrm{U}_{\mathrm{z}}=\frac{\mathrm{u}_{*}}{\mathrm{\kappa}} \ln \left[\frac{\mathrm{z}}{\mathrm{z}_{0}}+\Xi\right]
$$


where $U_{z}$ is the wind speed at elevation $z, u_{*}$ is the friction velocity, $\kappa=0.40$ is the von Karman constant, $z_{0}$ is the sea surface roughness length scale and $\Xi$ is a term that accounts for the effect of atmospheric stability on the wind speed profile. Since $\mathrm{u}_{*}$ was not directly measured in the experiment, its value was inferred from $U_{28}$.

To evaluate $\Xi$ using the bulk aerodynamic method (Large \& Pond, 1981), measurements of the sea surface temperature and the humidity are required. Unfortunately, the instruments for measuring both parameters failed to operate during the experiment so we were unable to evaluate this term. Large \& Pond (1981) showed that assuming neutral stability $(\Xi=0)$ introduces an uncertainty of less than $4 \%$ in the value of $u_{*}$ and $U_{z}$. We neglected the effect of atmospheric stability on the wind speed profile and used the equation

$$
\mathrm{U}_{\mathrm{z}}=\frac{\mathrm{u}_{*}}{\mathrm{\kappa}} \ln \frac{\mathrm{z}}{\mathrm{z}_{0}}
$$

Maat, Kraan \& Oost (1991) suggested that $\mathrm{z}_{0}$ is a function of the wave age $\left(\mathrm{c}_{\mathrm{p}} / \mathrm{u}_{*}\right)$ where $c_{p}$ is the phase speed of the peak frequency of the wave spectrum computed from the wave gauge record. Using an empirical fit to field data obtained during the 1986 Humidity Exchange Over the Sea Main Experiment [HEXMAX], they proposed that

$$
\mathrm{z}_{0}=0.8 \frac{\mathrm{u}_{*}^{2}}{\mathrm{~g}}\left(\frac{\mathrm{u}_{*}}{\mathrm{c}_{\mathrm{p}}}\right)
$$

From the two equations (Equations 4.2 and 4.3), we can determine the value of the two unknowns $\mathrm{u}_{*}$ and $\mathrm{z}_{0}$. We computed $\mathrm{u}_{*}$ and $\mathrm{z}_{0}$ by assuming a value for $\mathrm{u}$ in 

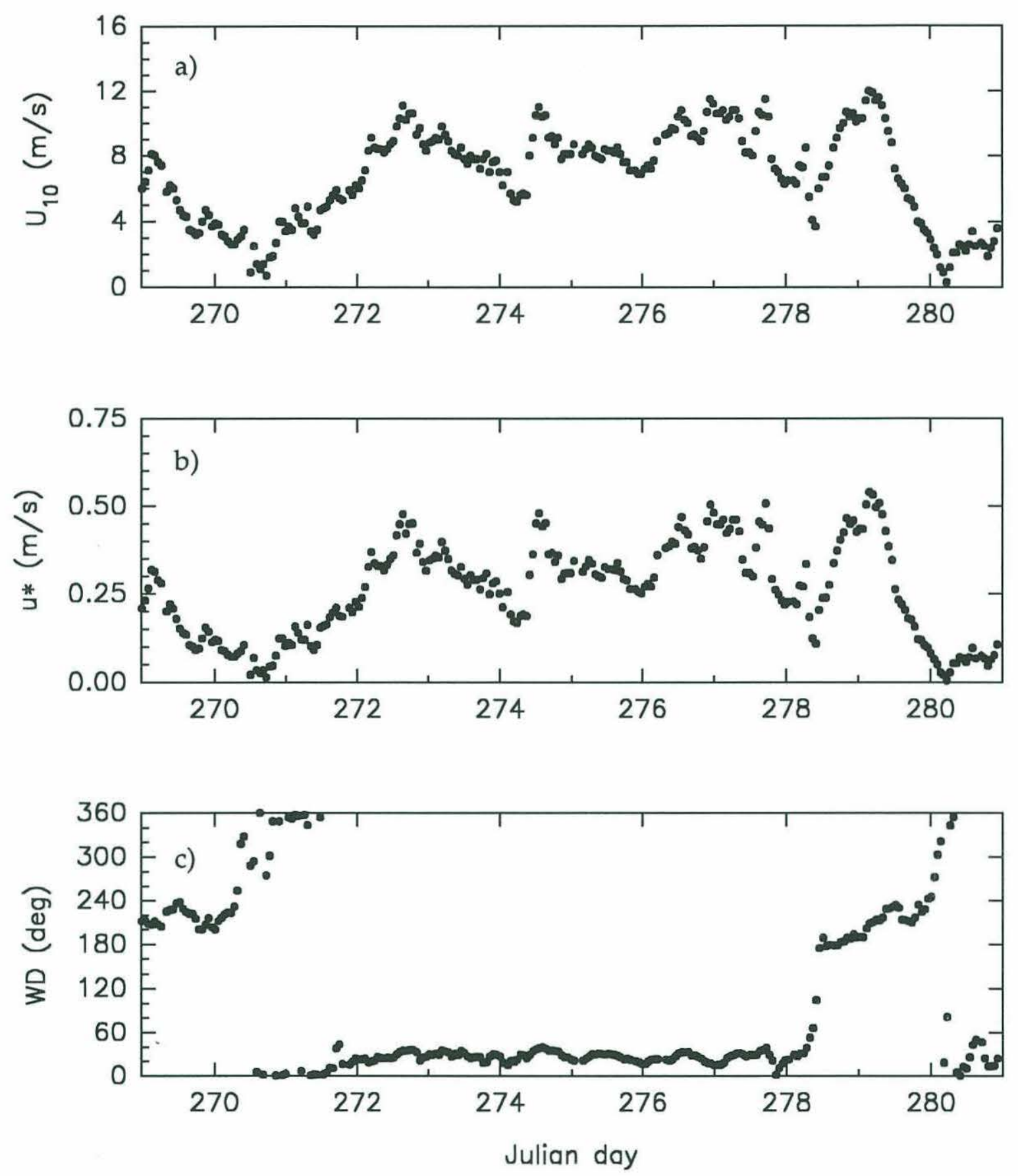

Figure 4.1. Time series of $\mathrm{U}_{10}, \mathrm{u}_{*}$ and the wind direction during the NOBS experiment. Note that the wind rapidly changes direction on Julian day 278 after blowing from the North for approximately six days. 


\section{Chapter 4}

Equation 4.3 and then substituting the computed value of $z_{0}$ into Equation 4.2 to get a new $\mathrm{u}$ * estimate. The process was repeated until the difference between the estimated and computed values for $u_{*}$ differed by less than $0.001 \mathrm{~m} / \mathrm{s}$. The value of $U_{10}$ was then computed from Equation 4.2. Figure 4.1 shows time series of hour-averaged $U_{10}$, $u_{*}$ and the wind direction during the observation period.

\subsection{Wave spectrum characteristics}

As we noted in Section 3.5, the wave gauge record was contaminated by low frequency energy due to the motion of the platform. Figure 3.12 shows that a significant proportion of this energy can be eliminated by specifying a high pass frequency cutoff at $0.05 \mathrm{~Hz}$, and that the contribution of the platform motion to the wave gauge record above $0.05 \mathrm{~Hz}$ is negligible compared to the wave energy. Daily plots of the one hour wave height spectrum for the time series beginning at 0000 UT throughout the observation period are shown in Figure 4.2.

The time series of the integral characteristics of the wave spectrum are shown in Figure 4.3. The root-mean square wave amplitude $a$ and the mean frequency $\overline{\mathrm{f}}$ of the wave spectrum were defined as

$$
\begin{aligned}
& a=\left[\int_{\mathrm{f}_{0}}^{\infty} \Phi(\mathrm{f}) \mathrm{df}\right]^{\frac{1}{2}} \\
& \overline{\mathrm{f}}=\frac{\int_{\mathrm{f}_{0}}^{\infty} \Phi(\mathrm{f}) \mathrm{fdf}}{a^{2}} .
\end{aligned}
$$




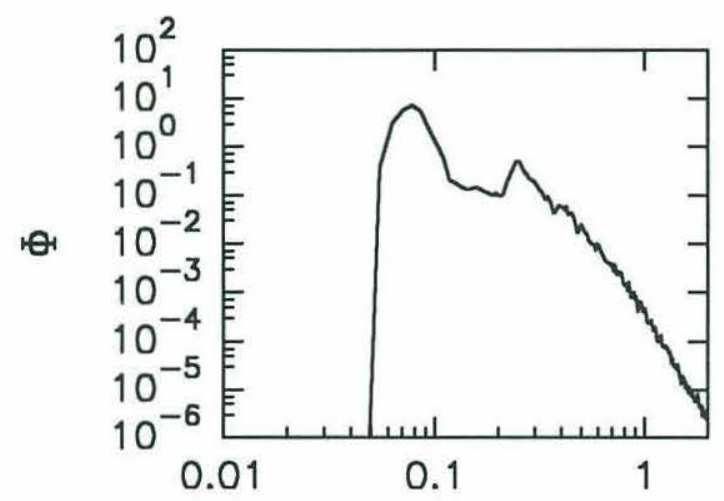

a) 269

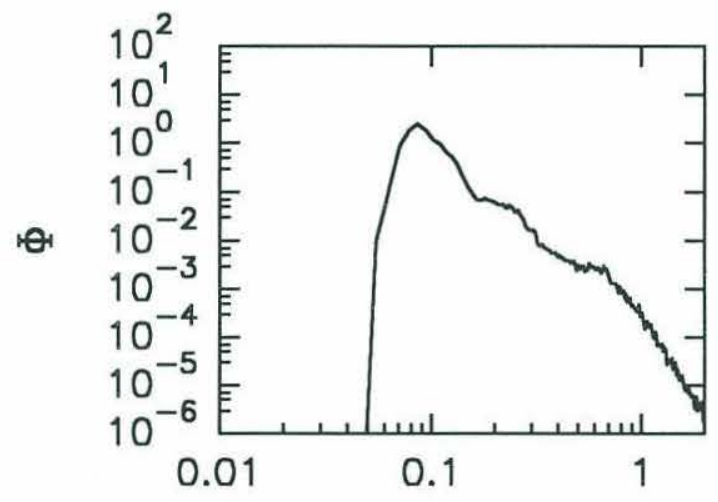

c) 271

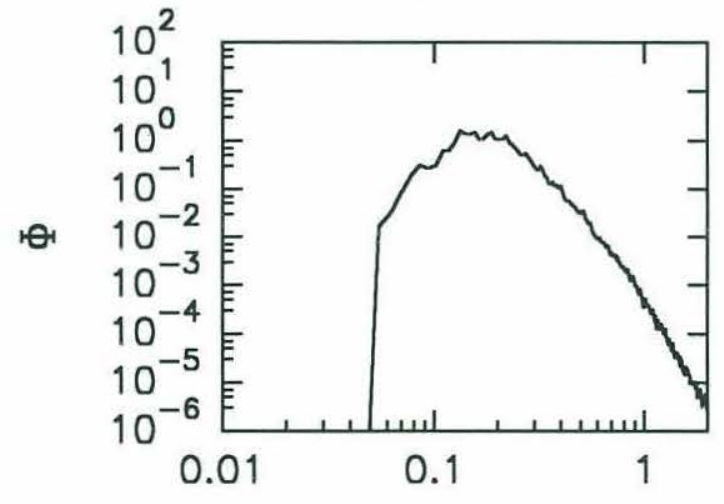

e) 273

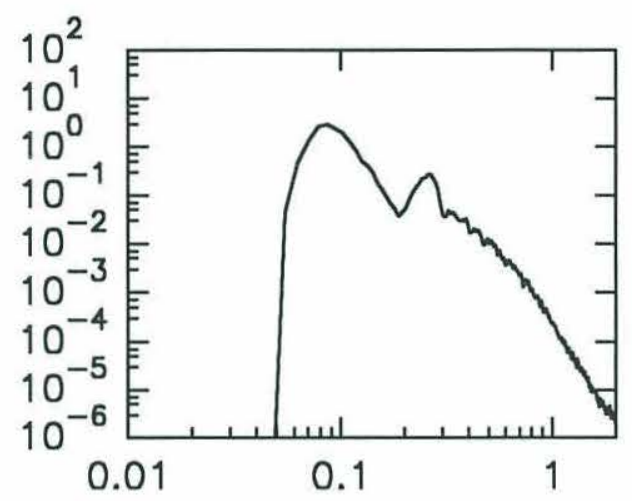

b) 270

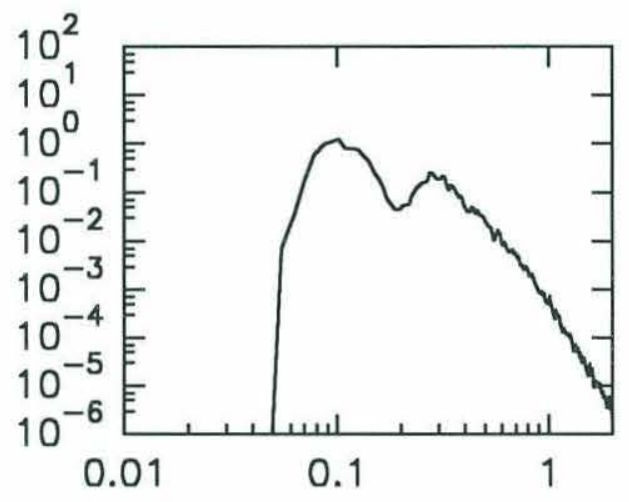

d) 272

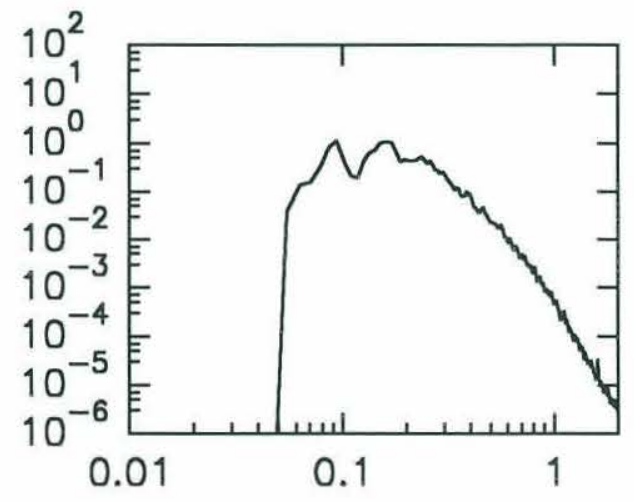

f) 274

Figure 4.2. Some typical 512-point wave height spectra $\Phi(\mathrm{f})\left[\mathrm{m}^{2} / \mathrm{Hz}\right]$ taken from one hour records at the start of each day of the NOBS experiment. 


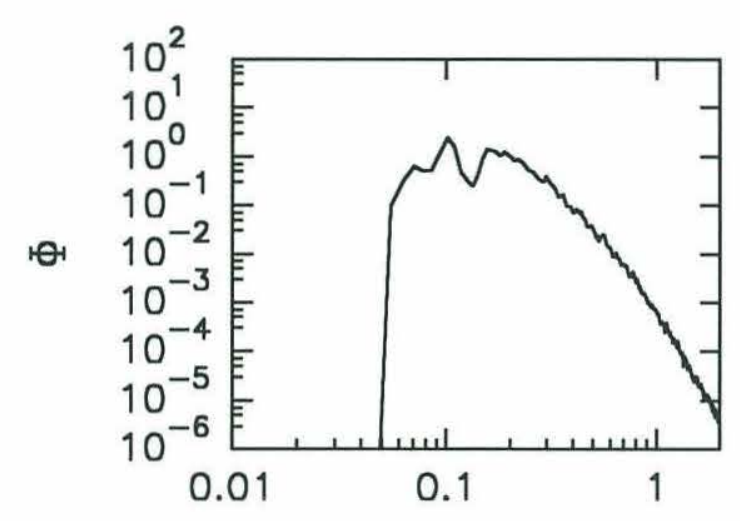

g) 275

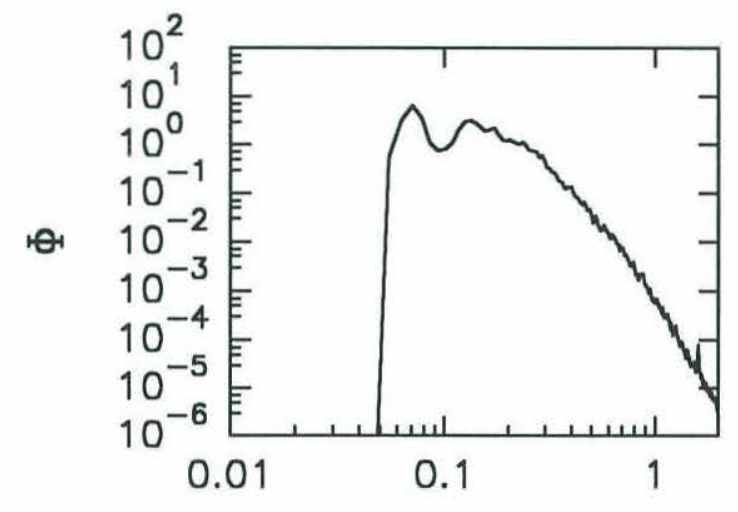

i) 277

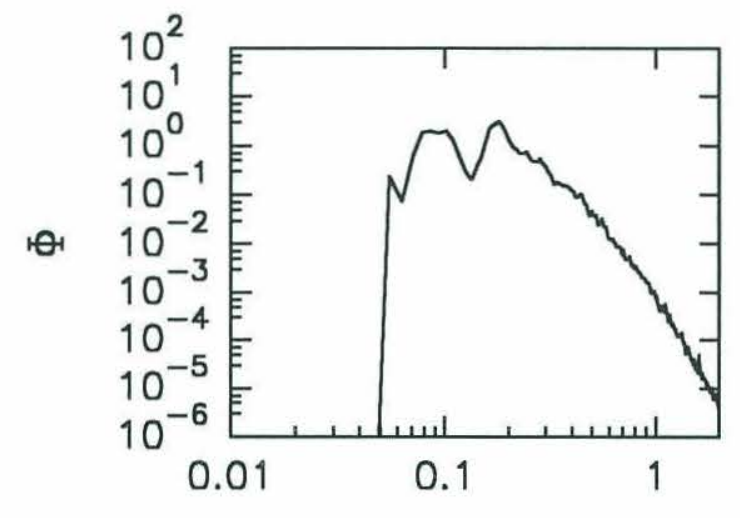

k) 279

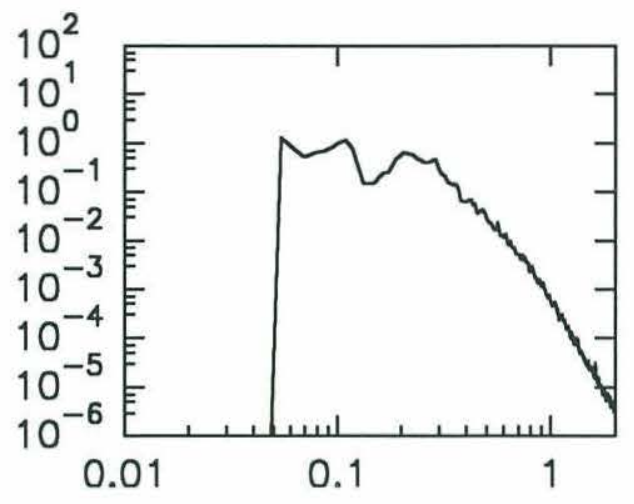

h) 276

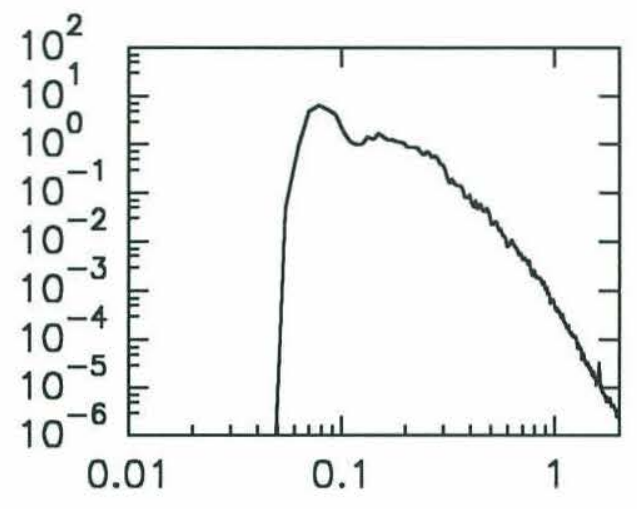

j) 278

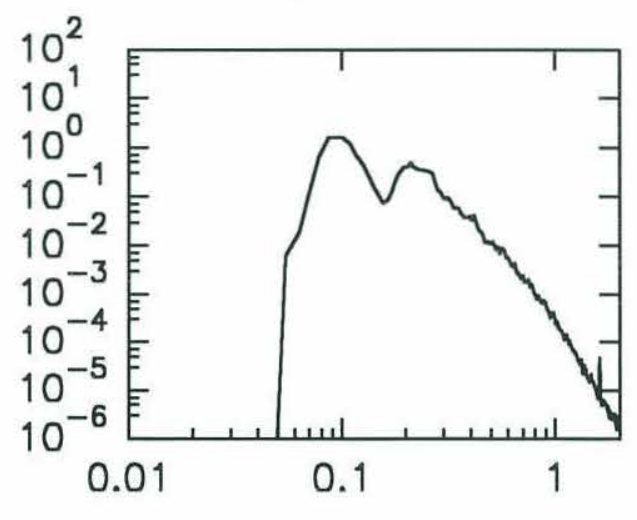

1) 280

Figure 4.2. Continued from last page. 

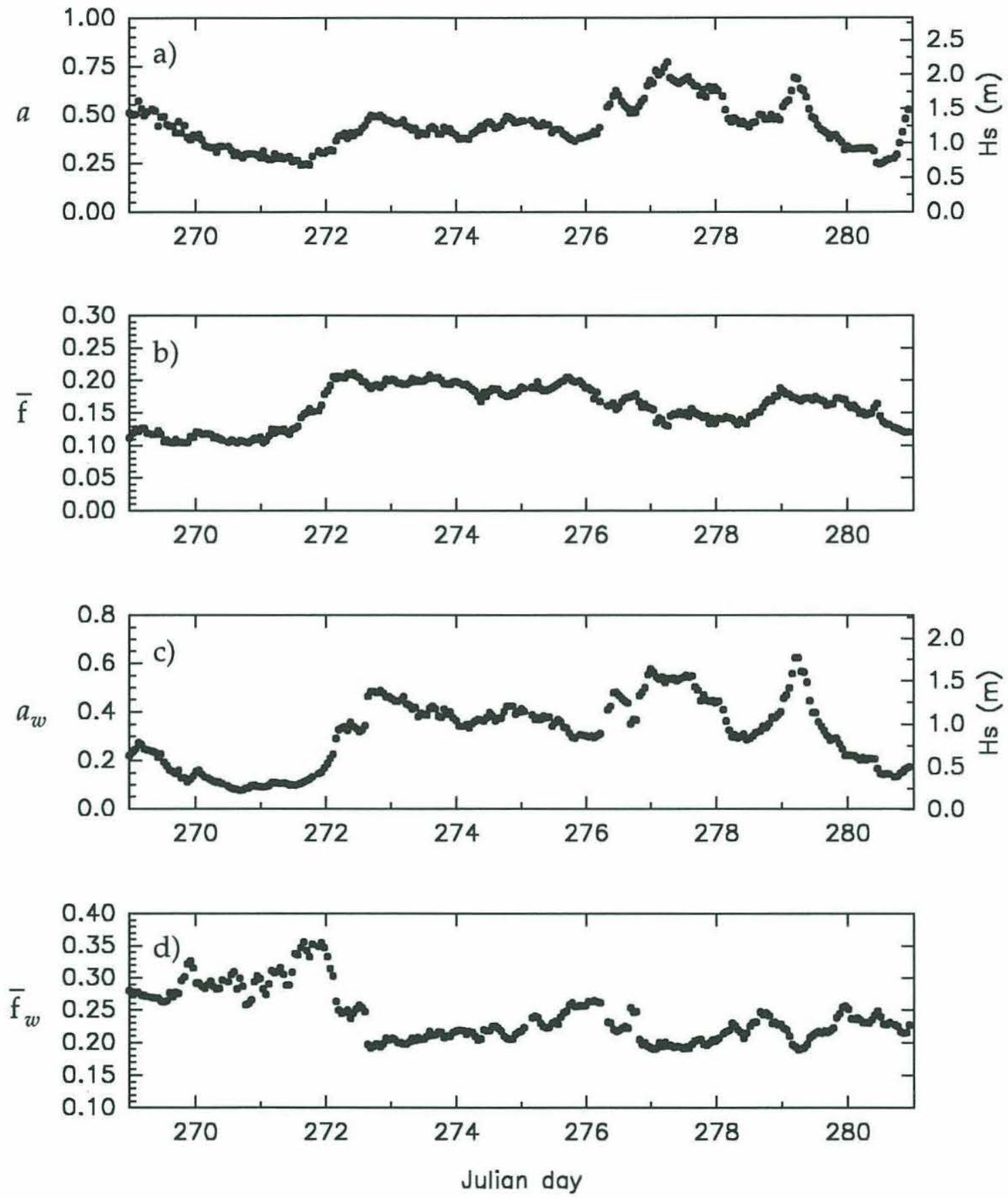

Figure 4.3. a) Root mean square amplitude $a(\mathrm{~m})$ and significant wave height $\mathrm{H}_{\mathrm{s}}(\mathrm{m})$ computed from the full spectrum. b) Mean frequency computed from the full spectrum $\overline{\mathrm{f}}(\mathrm{Hz}) . \mathrm{c}) a_{w}(\mathrm{~m})$ and $\mathrm{H}_{\mathrm{s}}(\mathrm{m})$ computed with swell filtered out. d) Mean frequency with swell filtered out $\bar{f}_{w}(\mathrm{~Hz})$. 


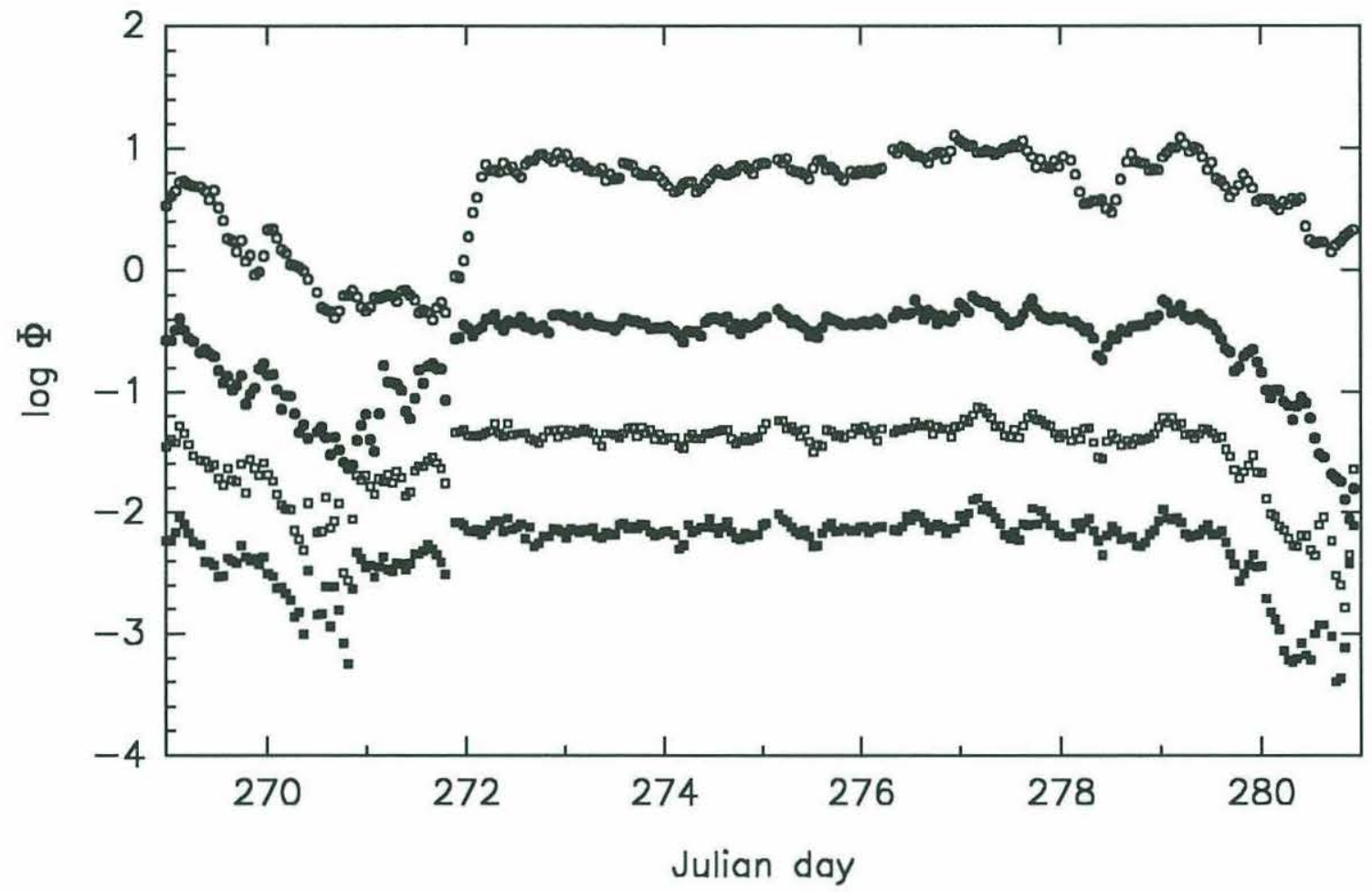

Figure 4.4. Time series of $\log \Phi\left(\mathrm{m}^{2} / \mathrm{Hz}\right)$ at selected frequencies: $0.25 \mathrm{~Hz}-0,0.5 \mathrm{~Hz}$ $\bullet, 0.75 \mathrm{~Hz}-\mathrm{a}, 1 \mathrm{~Hz}-\mathbf{\square}$. 


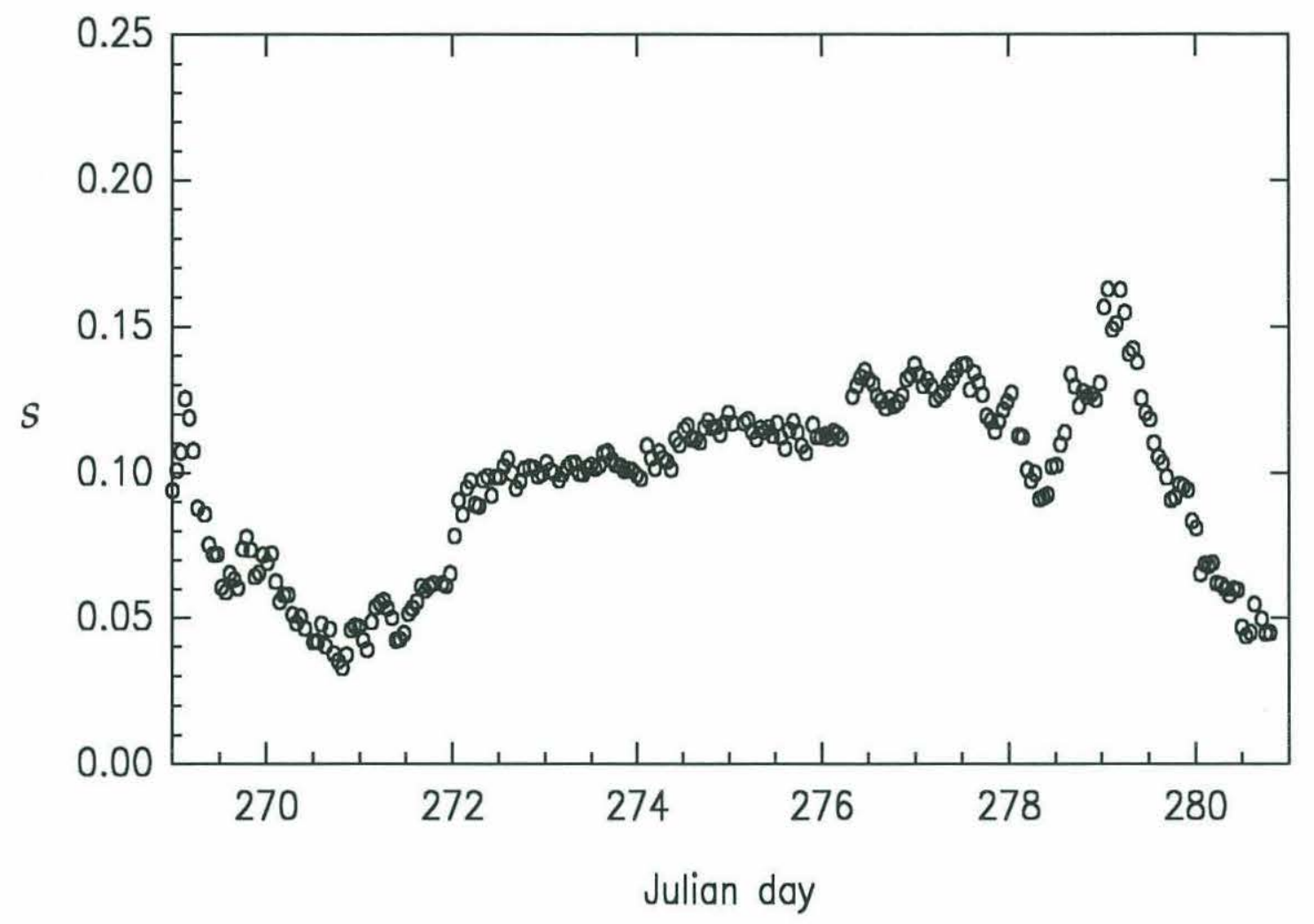

Figure 4.5. Time series of wave slope $s$ (Equation 3.12) computed from the resistance wire wave gauge array data. 
In Figures $4.3 \mathrm{a}$ and $4.3 \mathrm{~b}$, the lower limit of integration in Equations 4.4 and 4.5 was set at $\mathrm{f}_{0}=0.05 \mathrm{~Hz}$. The values of $a$ and $\overline{\mathrm{f}}$ in both plots include spectral energy from both wind waves and swell. In Figures $4.3 \mathrm{c}$ and $4.3 \mathrm{~d}$, the lower limit of integration used was the frequency between the swell and the wind sea peak in which $\Phi(f)$ was a minimum. This was chosen so that the energy of the swell does not affect the value of $a_{w}$ and $\overline{\mathrm{f}}_{\mathrm{w}}$. The plot shows that the wind wave spectrum $\overline{\mathrm{f}}_{\mathrm{w}}$ ranged from 0.15 to $0.4 \mathrm{~Hz}$ during NOBS.

Figures $4.3 \mathrm{a}$ and $4.3 \mathrm{c}$ also shows the wave height data in terms of the significant wave height $\mathrm{H}_{\mathrm{s}}$ defined as the mean of the highest one-third of waves. For a Rayleighdistributed wave (Kinsman, 1984, p. 390),

$$
\mathrm{H}_{s}=2.83 a \text {. }
$$

Figure 4.4 shows time series of the spectral level of $\Phi$ at $0.25,0.5,0.75$ and $1.0 \mathrm{~Hz}$. The plots in Figure 4.4 are a measure of the variability of the wave energy above the wind peak.

The method for computing the root mean square wave slope $s$ using the 4-wire wave gauge array was described in Section 3.6. The time series of $s$ is shown in Figure 4.5.

\subsection{Ambient noise}

Figure 4.6 presents the time series of the hour-averaged noise spectrum level $\mathrm{N}$ at selected frequencies [see Figure 3.11 for plots of typical N(f)]. We chose to display N at $4.3,8.0$, and $14.0 \mathrm{kHz}$ since these are the frequencies WOTANs commonly used (see 

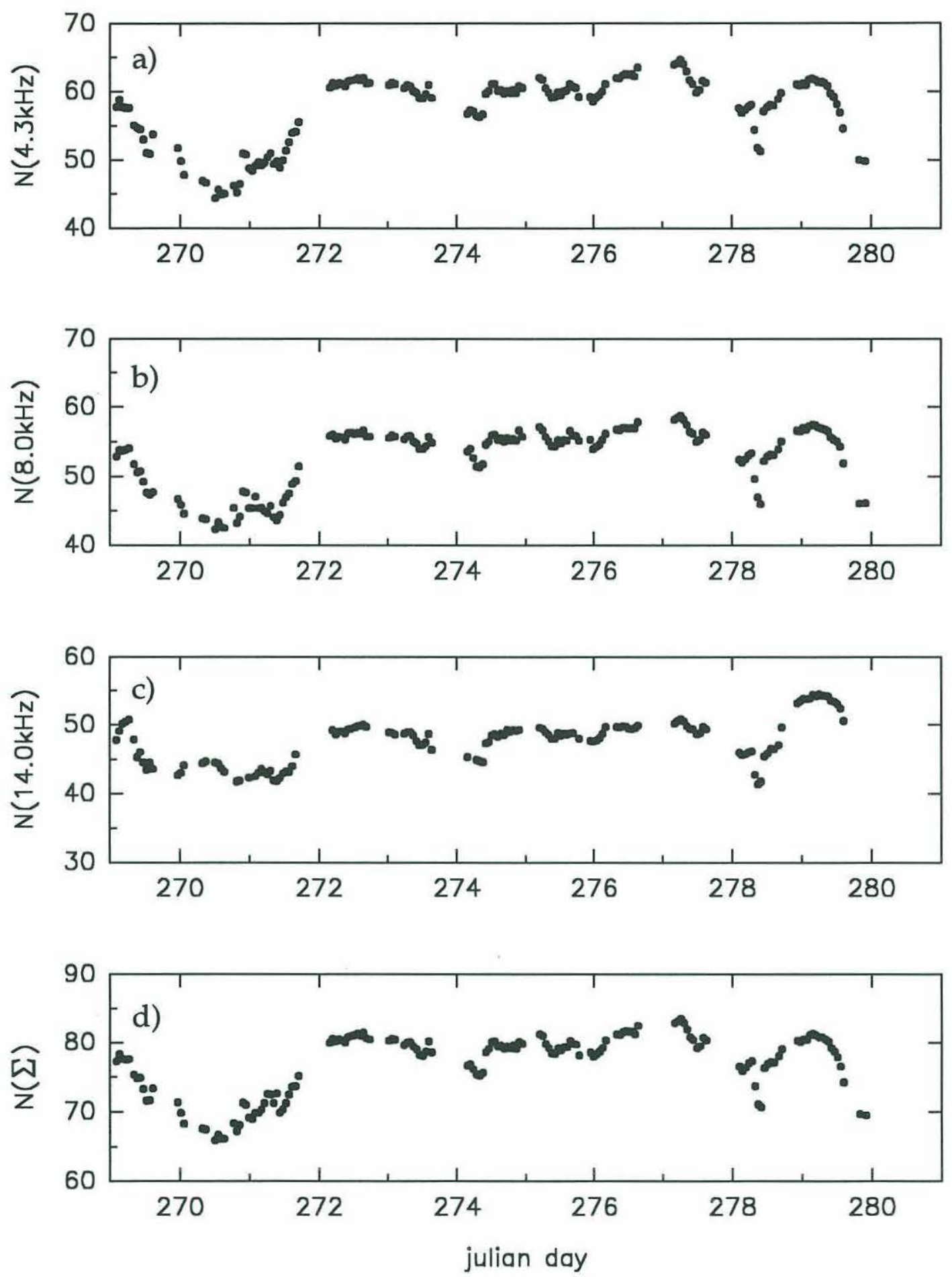

Figure 4.6. Time series of the ambient noise spectrum level $\mathrm{N}\left(\mathrm{dB}\right.$ re $\left.1 \mu \mathrm{Pa}^{2} / 1 \mathrm{~Hz}\right)$ at selected WOTAN frequencies. Note the similarities between these time series and the $\mathrm{U}_{10}$ time series. In particular, note the rapid drop and increase in $\mathrm{N}$ on Julian day 278.

a) $4.3 \mathrm{kHz}$. b) $8.0 \mathrm{kHz}$. c) $14.0 \mathrm{kHz}$. d) wide band [2.5-14 kHz or $\Sigma$ ]. 
Table 1.2). The total integrated acoustic energy between $2.5-14 \mathrm{kHz}$ denoted by $\Sigma$ is also presented. The gaps in the acoustic time series are due to records eliminated because some of the devices used in deploying instruments unrelated to our experiment generated electrical noise that contaminated the full acoustic spectrum.

Figures 4.1 and 4.5 show that the fluctuation in the wind speed $U_{10}$ and $u_{*}$ is clearly reflected in $\mathrm{N}$. The high correlation between $\mathrm{U}_{10}$ and $\mathrm{N}$ is consistent with the investigations we reviewed in Section 1.1. In addition, good correlations between $\mathrm{N}$ and properties of the wave height spectrum $\Phi$, particularly $a_{w}$, the high frequency wave spectrum components in Figure 4.4 and wave slope $s$ in Figure 4.5, and $U_{10}$ and $\mathrm{N}$ is also apparent in Figures $4.3-4.6$. This will be clearer in Section 4.6 when we examine the empirical relationships between $\mathrm{N}$ and environmental parameters.

\subsection{Directional wave height spectra.}

The method for computing the directional wave height spectra was outlined in Section 3.7. The maximum horizontal dimension of the array is 1 meter. The smallest wavelength the array can resolve is of this order. Consequently, the highest wave frequency that can be resolved is approximately $1 \mathrm{~Hz}$.

Figure 4.1 shows that although the wind speed has fluctuated between 5 to 11 meters/second, the wind direction has been quasi-steady (from approximately $30^{\circ}$ ) for 6 days prior to Julian day 278. The wind then began to change direction and die down with $\mathrm{U}_{10}$ decreasing to 4 meters/second while turning. After several hours, the wind had completely turned and was blowing from the South $\left(180^{\circ}\right)$.

The evolution of the wave field during this event is shown by a series of contour plots of directional wave spectra $\Phi(f, \theta)$ in Figure 4.7. The plots have a directional resolution 
of $5^{\circ}$ and a frequency resolution of $0.002 \mathrm{~Hz}$. They show the directional distribution of the wave energy within the frequency range 0.25 to $1 \mathrm{~Hz}$. Since the wave energy spectrum $\Phi(\mathrm{f})$ decays as a function of frequency, only the wave energy at the lower frequency limit are seen in these plots. In addition, since the directional wave spectra plots show the direction the waves are moving to while the anemometer gives the direction the wind is blowing from, we added $180^{\circ}$ to the wind direction to facilitate comparison. The figure lists the start time, the wind speed and the direction the wind is blowing towards $\left(\theta_{\text {wind }}\right)$ for each plot.

The wind direction $\theta_{\text {wind }}$ and wind speed are relatively steady in the first six plots. The wind begins to turn and decay in Figure $4.7 \mathrm{~g}$ with $\mathrm{U}_{10}$ reaching a minimum in Figure 4.71. During this period, the mean wave direction in the energy containing portion of the spectrum and the wind direction are oriented in different directions. In Figure $4.7 \mathrm{n}$, we see some wave energy at approximately $350^{\circ}$ and $0.45 \mathrm{~Hz}$. This matches the wind direction for that plot which is at $354^{\circ}$. In the next plot, the energy at this direction has increased and begun to migrate towards the lower frequencies. In the remaining plots, the wave energy centered around the original direction $\left(200^{\circ}\right)$ has decayed considerably while the wave energy parallel to the wind has already grown significantly.

While these plots are informative, the information content is biased towards the lower frequencies because the energy level there is higher than at the higher frequencies. It is possible to reduce this bias and observe the high frequency directional behavior of the wave field by eliminating the influence of the spectral energy level on the plot. The same plots in Figure 4.7 were plotted in Figure 4.8. However, instead of equating the total wave energy at each frequency with the spectral energy level of the 


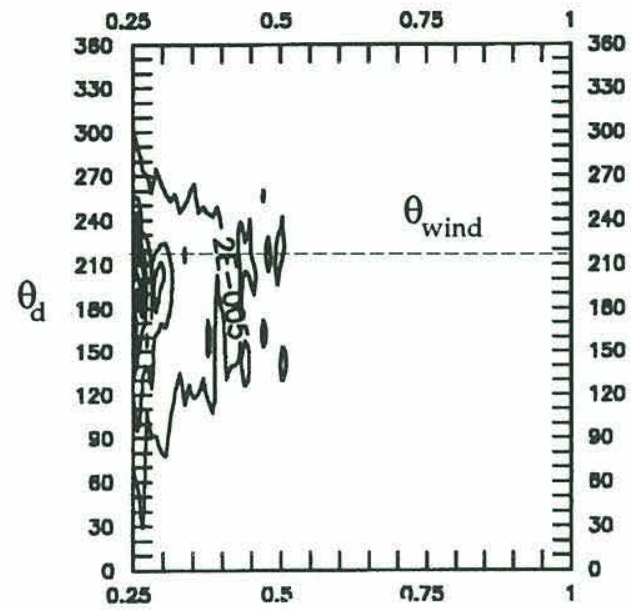

a

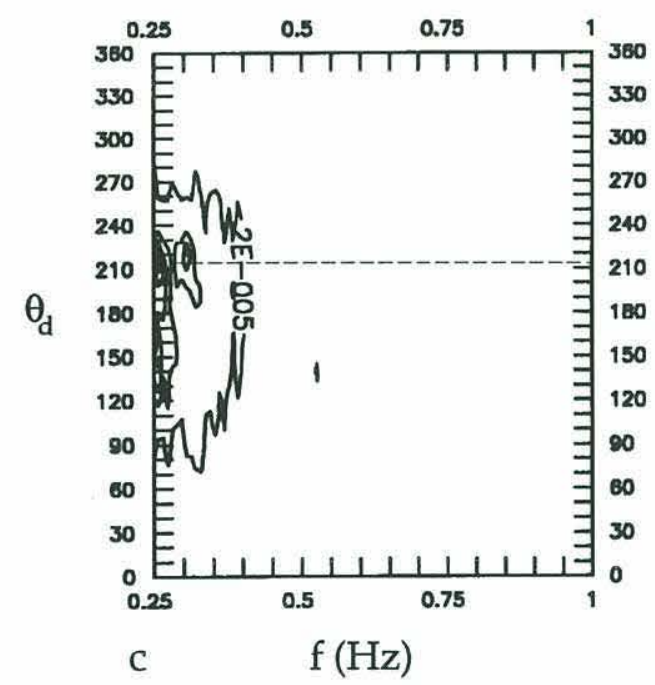

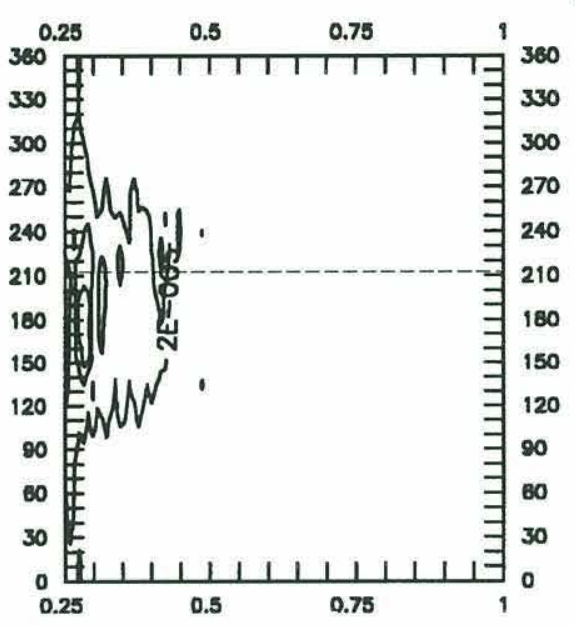

$\mathrm{b}$

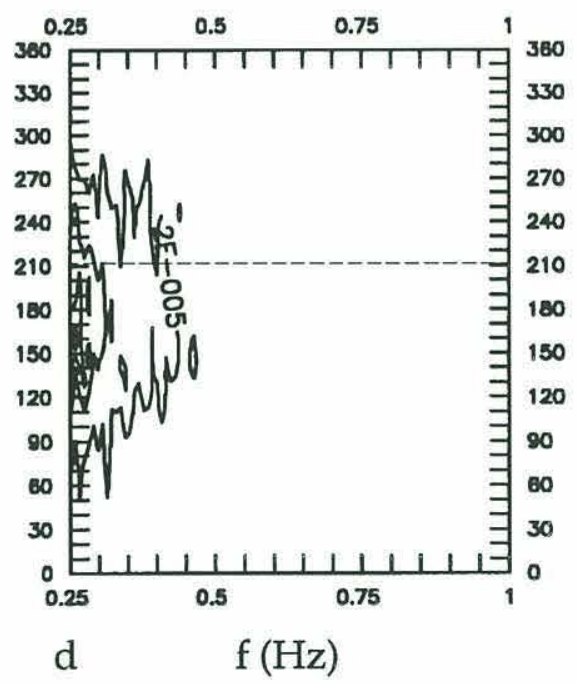

Figure 4.7. Directional wave spectra from 0.25 to $1 \mathrm{~Hz}$. The sequence of plots demonstrate the evolution of the wavefield in response to turning winds on Julian day 278. The $x$-axis for the plots is frequency in Hertz and the $y$-axis is wave direction $\theta_{\mathrm{d}}$. Dashed lines indicate the direction the wind is blowing towards $\left(\theta_{\text {wind }}\right)$.

\begin{tabular}{|c|c|c|c|}
\hline & Start Time (UT) & $\mathrm{U}_{10}(\mathrm{~m} / \mathrm{s})$ & $\theta_{\text {wind }}$ \\
\hline $\mathrm{a}$ & 0407 & 8.0 & $211^{\circ}$ \\
\hline $\mathrm{b}$ & 0439 & 8.1 & $211^{\circ}$ \\
\hline $\mathrm{c}$ & 0511 & 7.7 & $211^{\circ}$ \\
\hline $\mathrm{d}$ & 0543 & 8.2 & $212^{\circ}$ \\
\hline
\end{tabular}



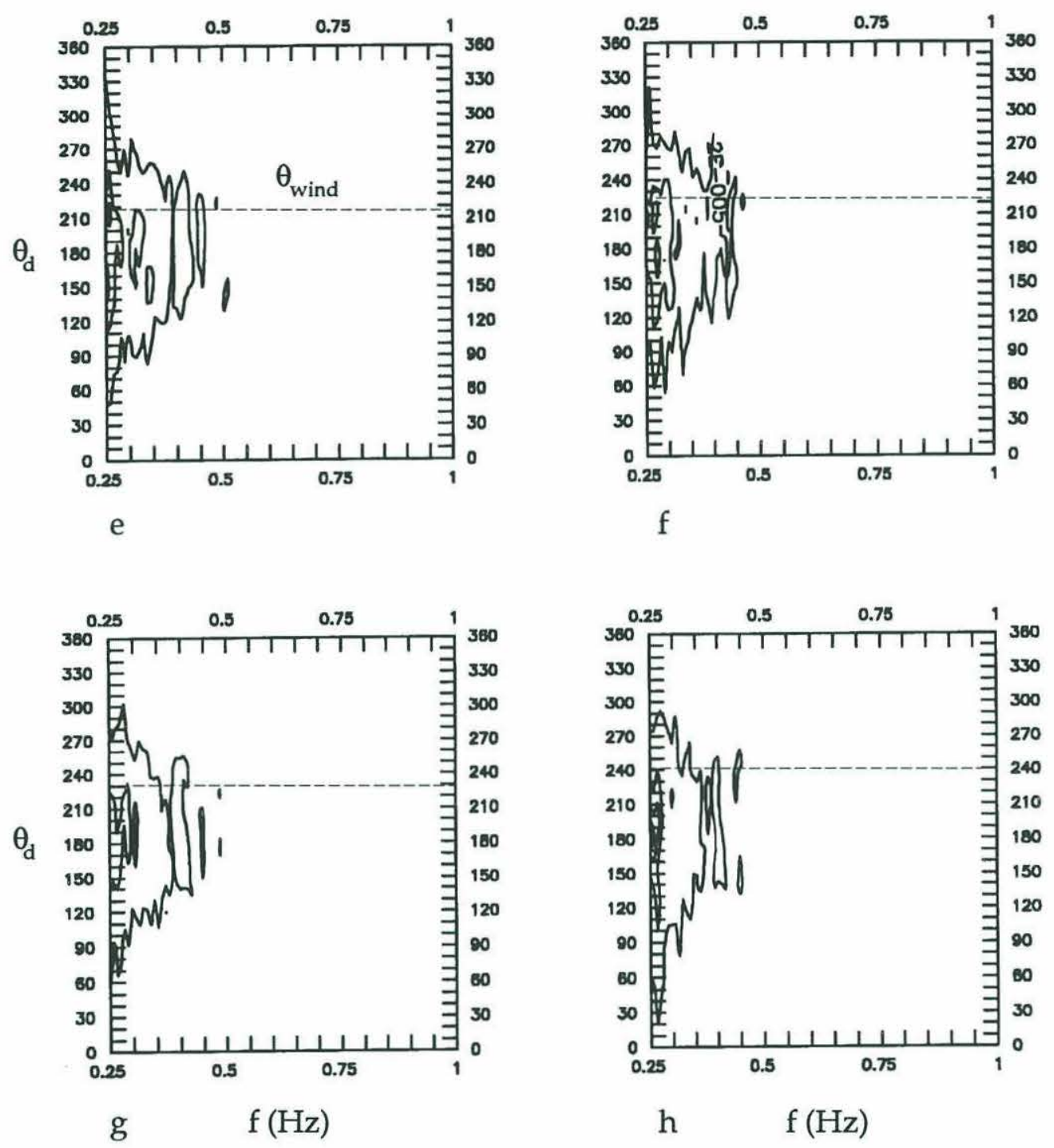

Figure 4.7. Continued from last page.

\begin{tabular}{|c|c|c|c|}
\hline & Start Time (UT) & $\mathrm{U}_{10}(\mathrm{~m} / \mathrm{s})$ & $\theta_{\text {wind }}$ \\
\hline $\mathrm{e}$ & 0615 & 9.1 & $214^{\circ}$ \\
\hline $\mathrm{f}$ & 0647 & 9.7 & $223^{\circ}$ \\
\hline $\mathrm{g}$ & 0719 & 6.9 & $228^{\circ}$ \\
\hline $\mathrm{h}$ & 0751 & 5.2 & $238^{\circ}$ \\
\hline
\end{tabular}



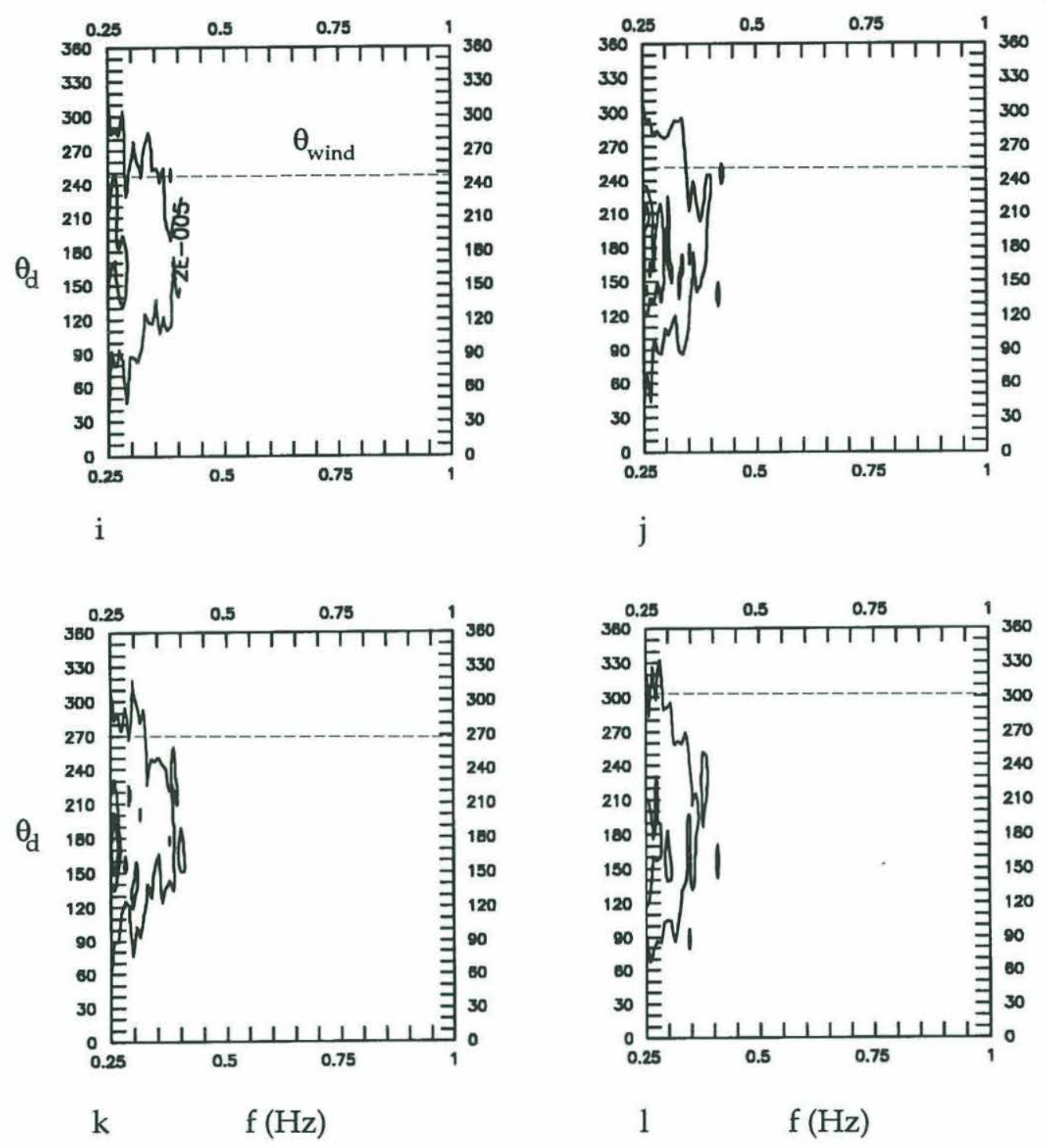

Figure 4.7. Continued from last page.

\begin{tabular}{|c|c|c|c|}
\hline & Start Time (UT) & $\mathrm{U}_{10}(\mathrm{~m} / \mathrm{s})$ & $\theta_{\text {wind }}$ \\
\hline $\mathrm{i}$ & 0823 & 4.6 & $244^{\circ}$ \\
\hline $\mathrm{j}$ & 0855 & 4.2 & $249^{\circ}$ \\
\hline $\mathrm{k}$ & 0927 & 4.3 & $267^{\circ}$ \\
\hline $\mathrm{l}$ & 0959 & 3.7 & $302^{\circ}$ \\
\hline
\end{tabular}




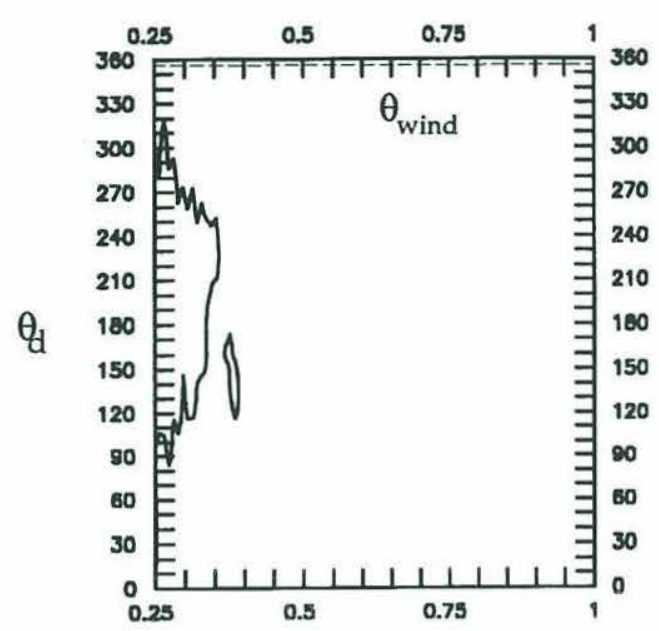

m

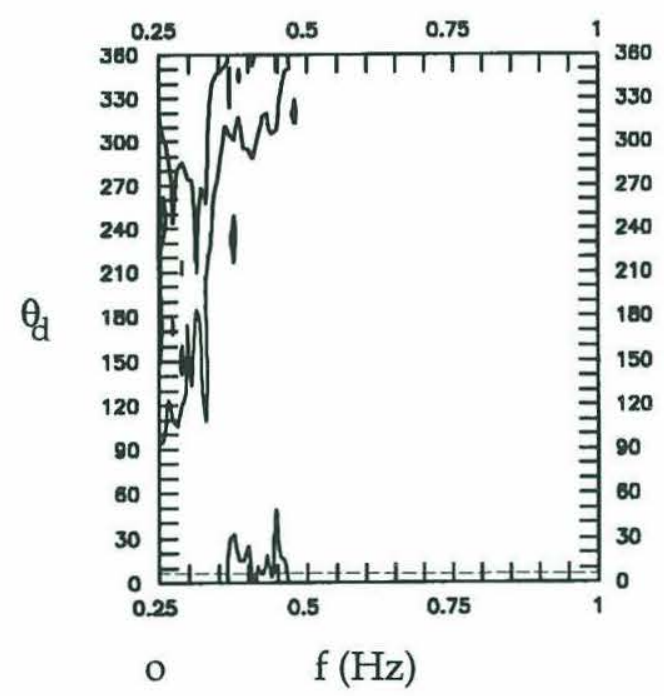

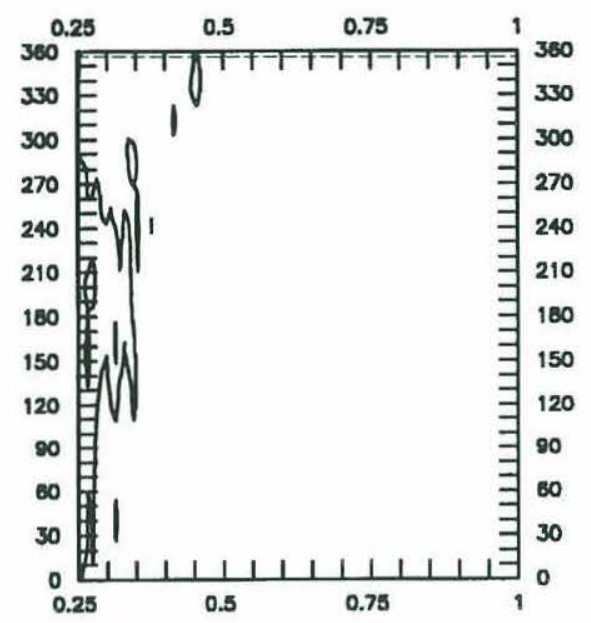

$\mathrm{n}$

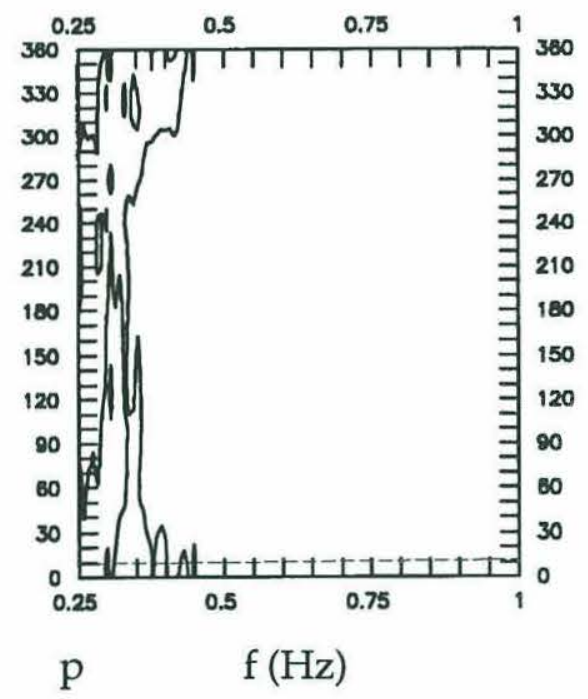

Figure 4.7. Continued from last page.

\begin{tabular}{|c|c|c|c|}
\hline & Start Time $(\mathrm{UT})$ & $\mathrm{U}_{10}(\mathrm{~m} / \mathrm{s})$ & $\theta_{\text {wind }}$ \\
\hline $\mathrm{m}$ & 1031 & 5.9 & $354^{\circ}$ \\
\hline $\mathrm{n}$ & 1103 & 6.7 & $355^{\circ}$ \\
\hline $\mathrm{o}$ & 1135 & 7.7 & $6^{\circ}$ \\
\hline $\mathrm{p}$ & 1207 & 7.0 & $9^{\circ}$ \\
\hline
\end{tabular}




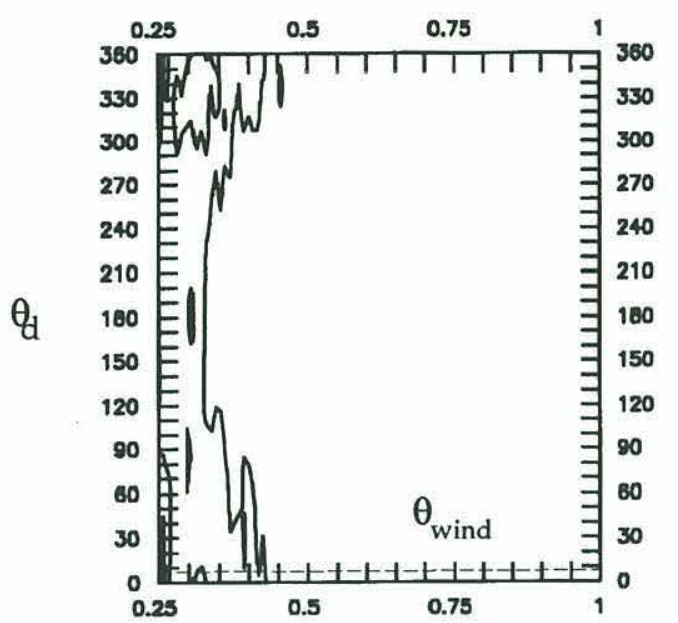

q

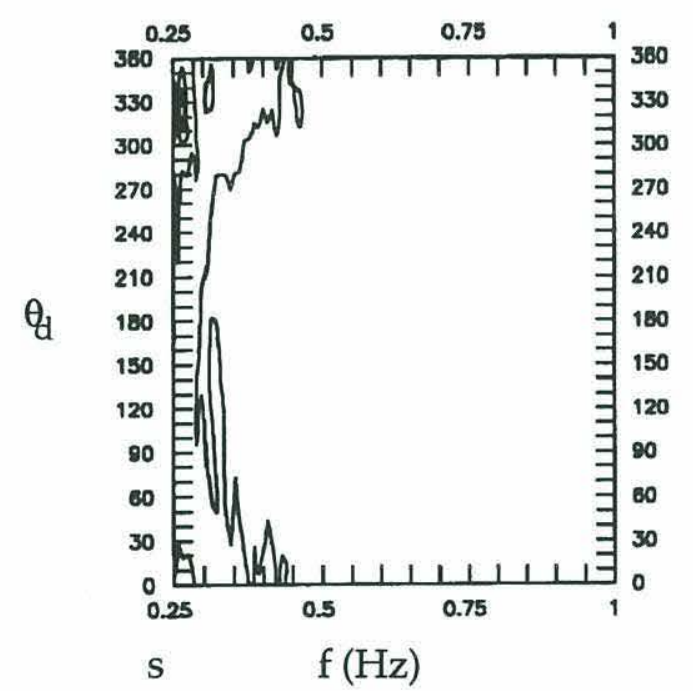

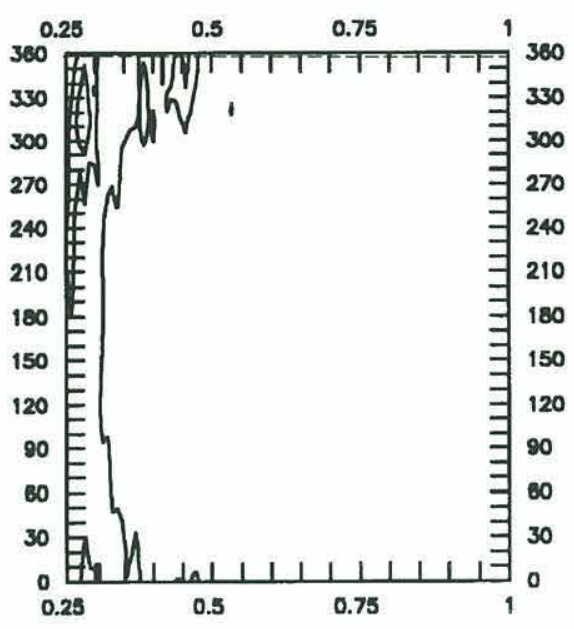

r

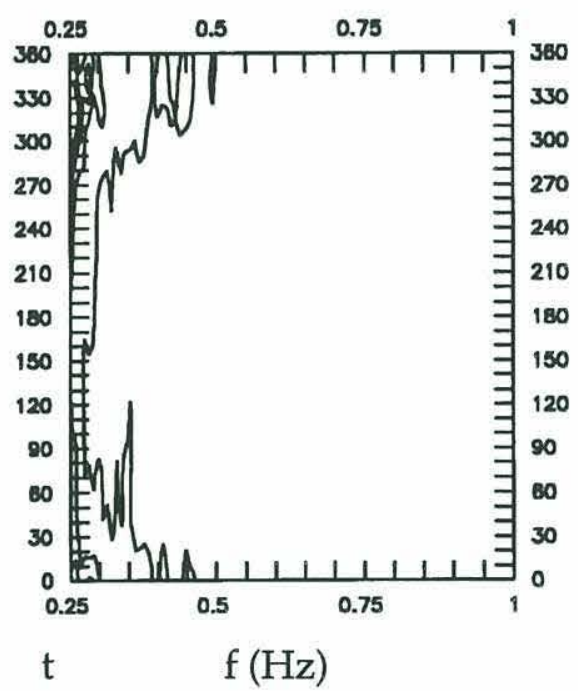

Figure 4.7. Continued from last page.

\begin{tabular}{|c|c|c|c|}
\hline & Start Time (UT) & $\mathrm{U}_{10}(\mathrm{~m} / \mathrm{s})$ & $\theta_{\text {wind }}$ \\
\hline $\mathrm{q}$ & 1239 & 7.0 & $3^{\circ}$ \\
\hline $\mathrm{r}$ & 1311 & 7.2 & $357^{\circ}$ \\
\hline $\mathrm{s}$ & 1343 & 7.9 & $358^{\circ}$ \\
\hline $\mathrm{t}$ & 1415 & 8.3 & $0^{\circ}$ \\
\hline
\end{tabular}



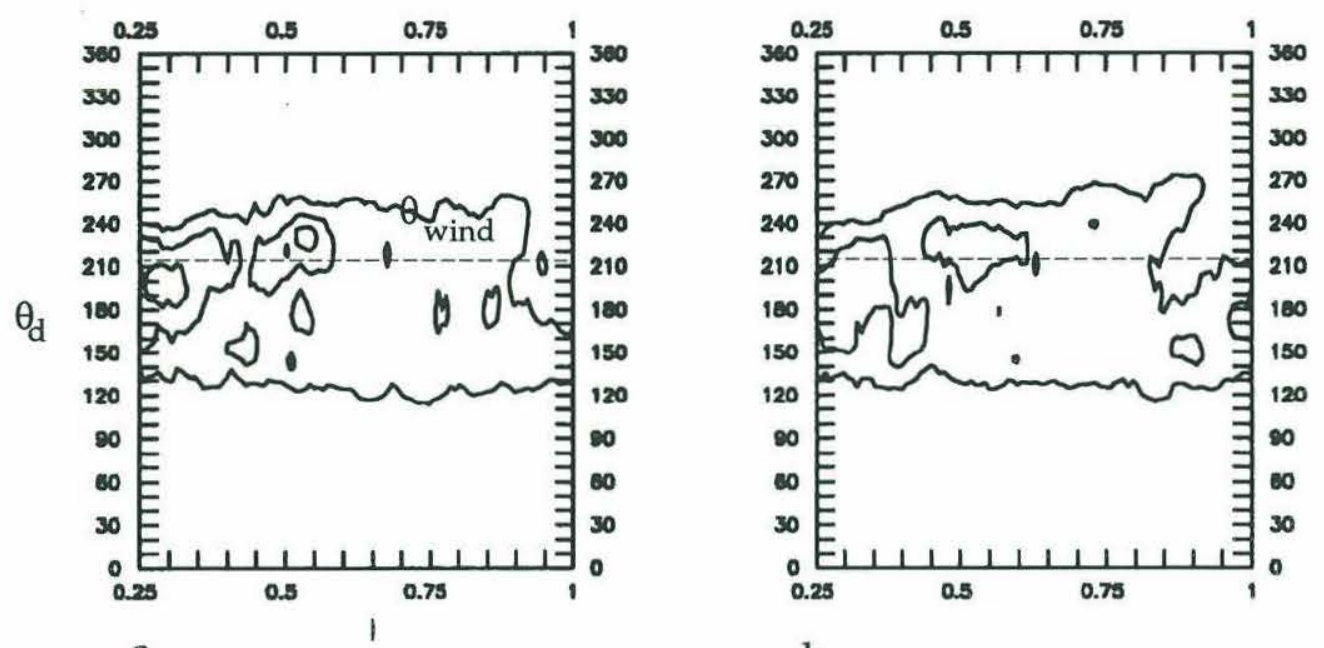

a

$\mathrm{b}$
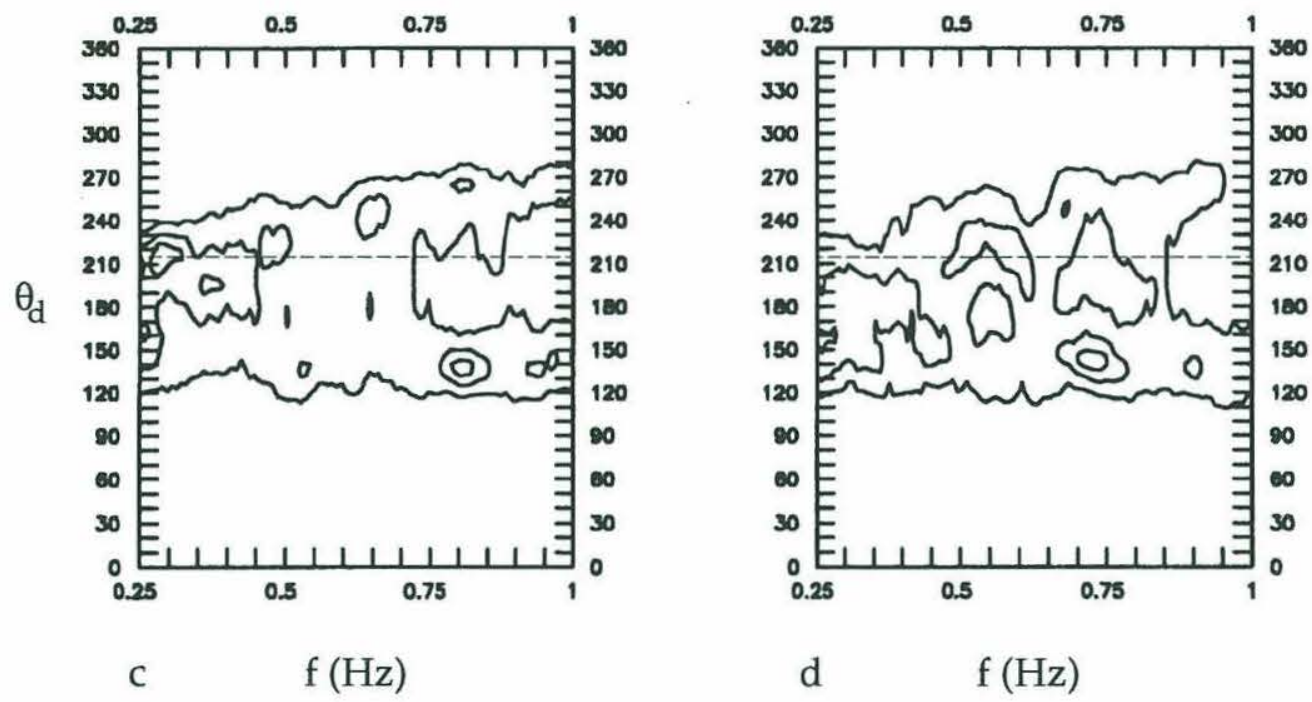

Figure 4.8. The directional wave spectra in Figure 4.7 normalized by the spectrum level at the given frequency to highlight the directional distribution of the wave energy in response to turning winds on Julian day 278. The $\mathrm{x}$-axis for the plots is frequency in Hertz and the $y$-axis is the wave direction $\theta_{d}$. Dashed lines indicate the direction the wind is blowing towards $\left(\theta_{\text {wind }}\right)$.

\begin{tabular}{|c|c|c|c|}
\hline & Start Time (UT) & $\mathrm{U}_{10}(\mathrm{~m} / \mathrm{s})$ & $\theta_{\text {wind }}$ \\
\hline $\mathrm{a}$ & 0407 & 8.0 & $211^{\circ}$ \\
\hline $\mathrm{b}$ & 0439 & 8.1 & $211^{\circ}$ \\
\hline $\mathrm{c}$ & 0511 & 7.7 & $211^{\circ}$ \\
\hline $\mathrm{d}$ & 0543 & 8.2 & $212^{\circ}$ \\
\hline
\end{tabular}



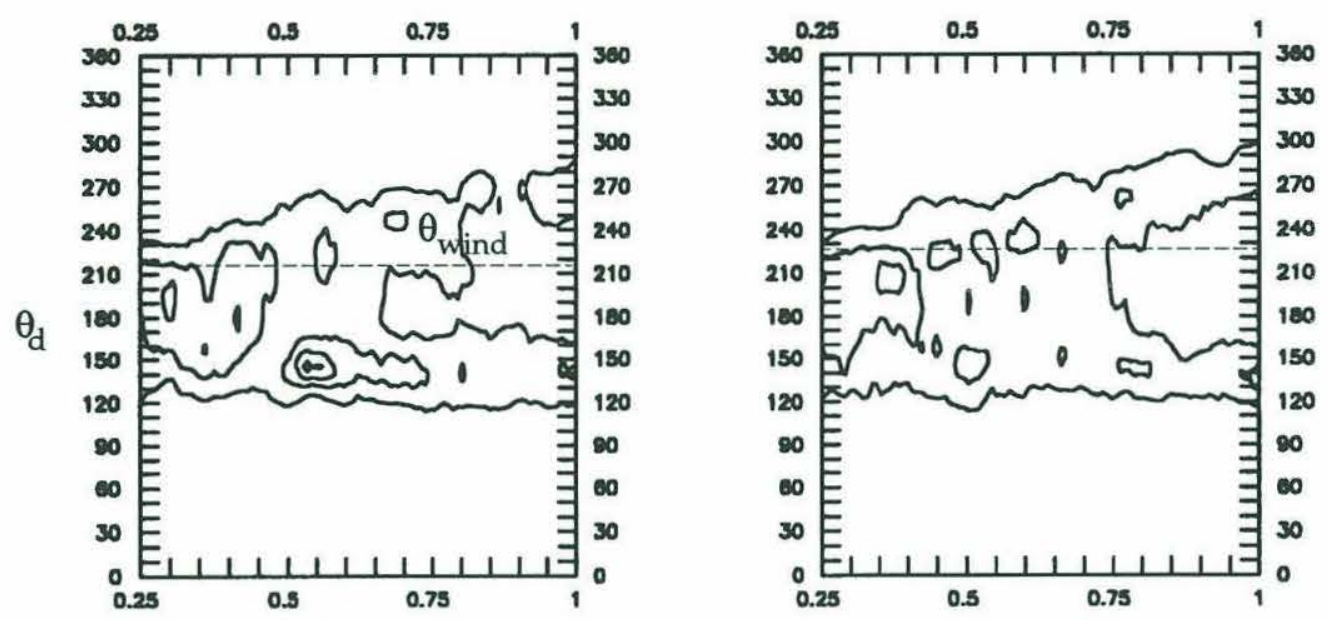

e
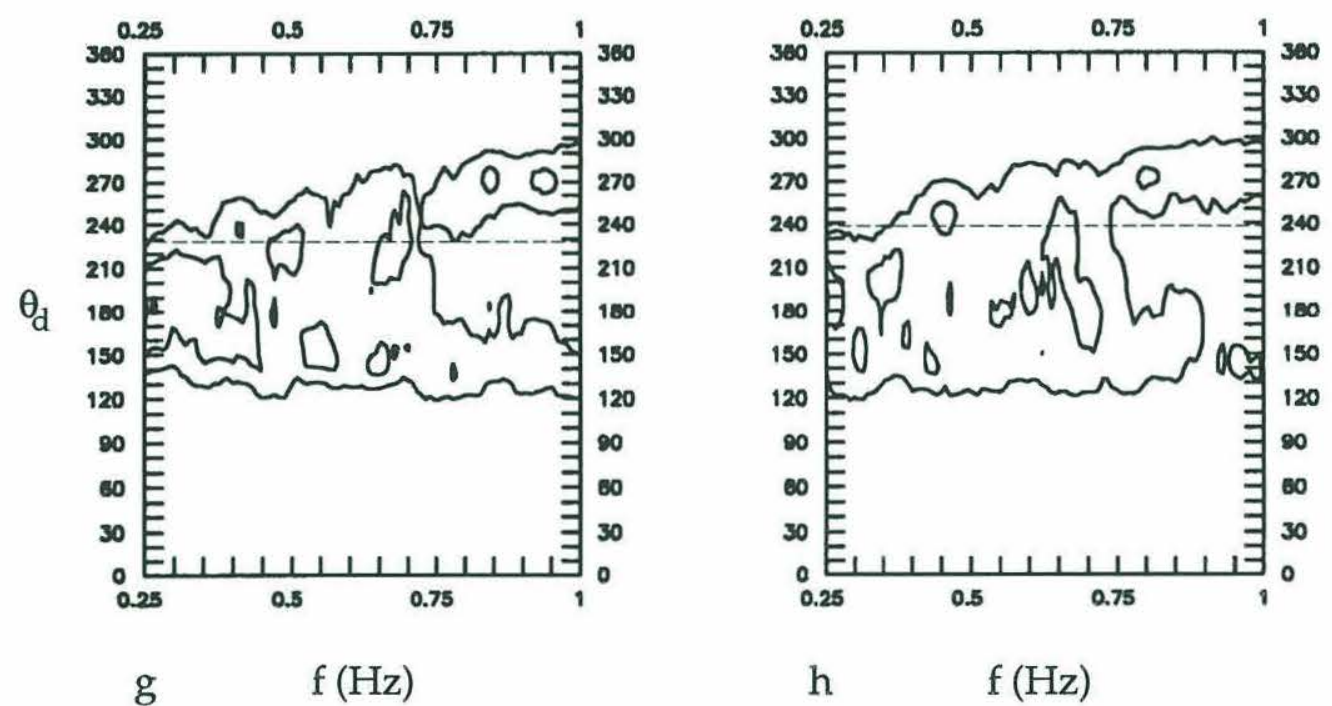

Figure 4.8. Continued from last page.

\begin{tabular}{|c|c|c|c|}
\hline & Start Time (UT) & $\mathrm{U}_{10}(\mathrm{~m} / \mathrm{s})$ & $\theta_{\text {wind }}$ \\
\hline $\mathrm{e}$ & 0615 & 9.1 & $214^{\circ}$ \\
\hline $\mathrm{f}$ & 0647 & 9.7 & $223^{\circ}$ \\
\hline $\mathrm{g}$ & 0719 & 6.9 & $228^{\circ}$ \\
\hline $\mathrm{h}$ & 0751 & 5.2 & $238^{\circ}$ \\
\hline
\end{tabular}



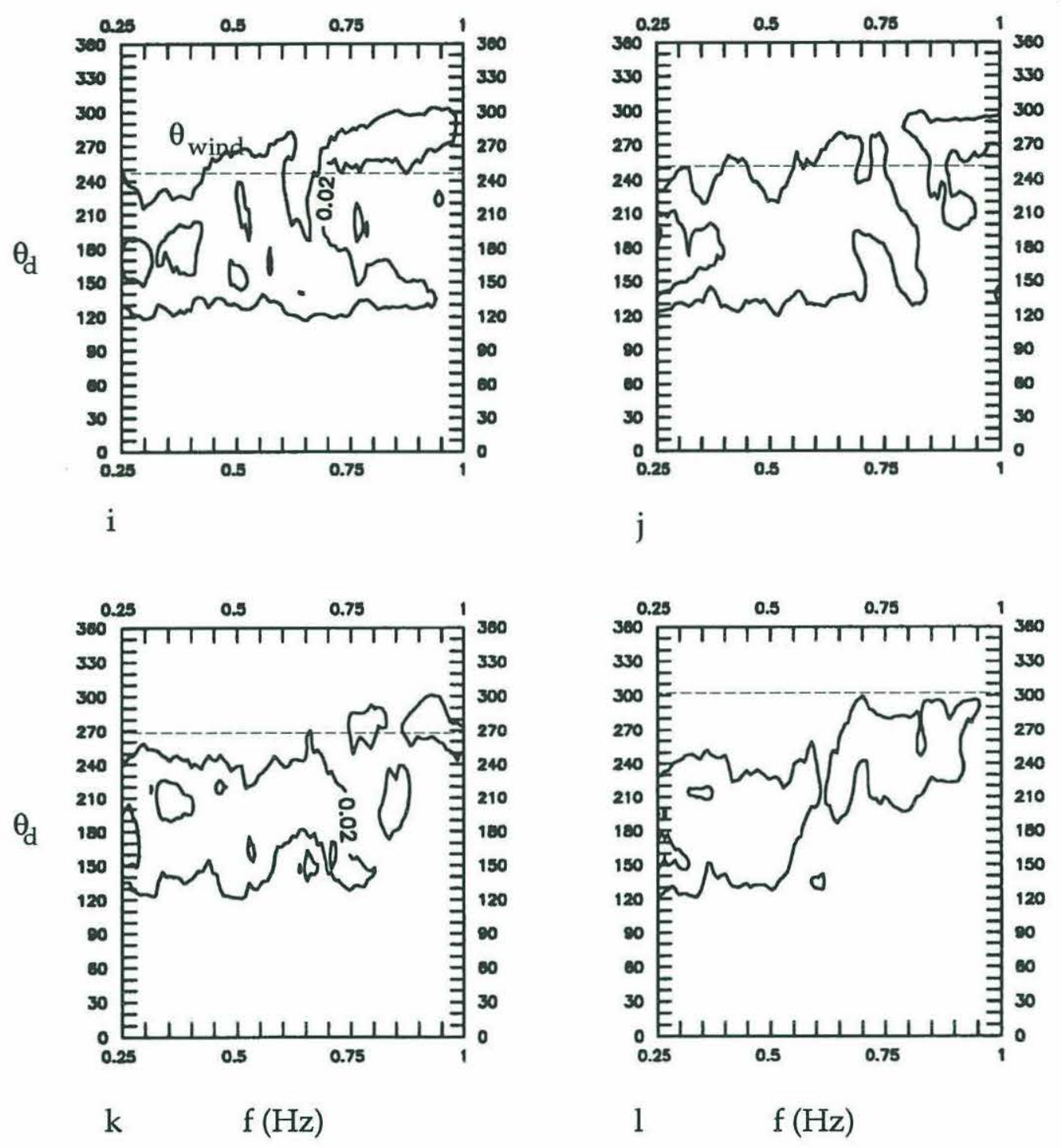

Figure 4.8. Continued from last page.

\begin{tabular}{|c|c|c|c|}
\hline & Start Time (UT) & $\mathrm{U}_{10}(\mathrm{~m} / \mathrm{s})$ & $\theta_{\text {wind }}$ \\
\hline $\mathrm{i}$ & 0823 & 4.6 & $244^{\circ}$ \\
\hline $\mathrm{j}$ & 0855 & 4.2 & $249^{\circ}$ \\
\hline $\mathrm{k}$ & 0927 & 4.3 & $267^{\circ}$ \\
\hline $\mathrm{l}$ & 0959 & 3.7 & $302^{\circ}$ \\
\hline
\end{tabular}




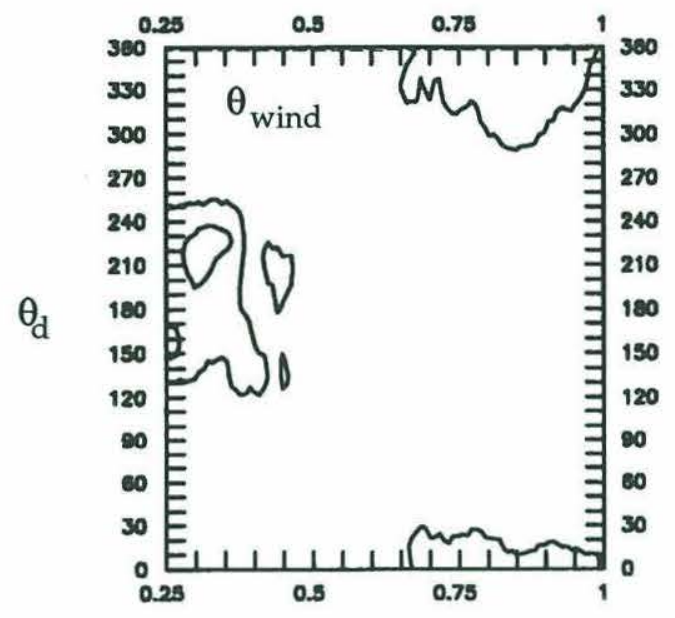

m

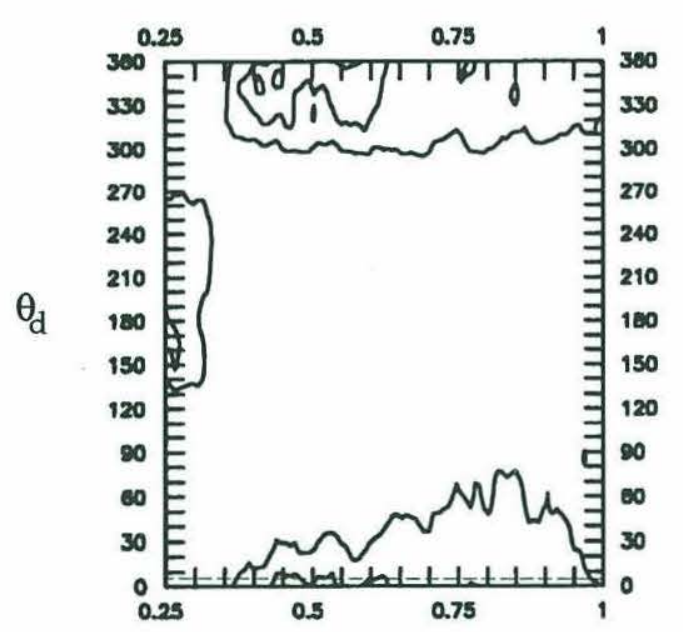

o $\quad \mathrm{f}(\mathrm{Hz})$

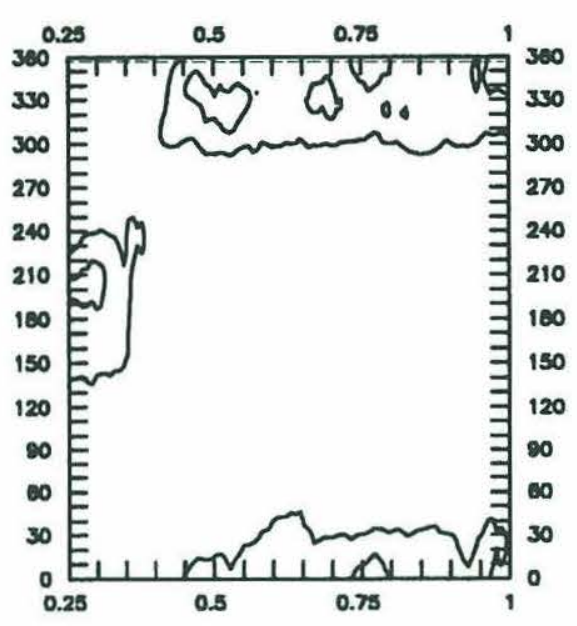

n

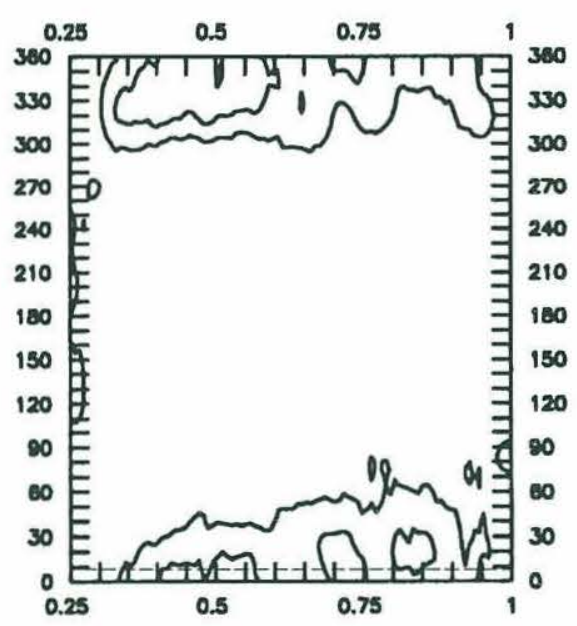

$\mathrm{p}$

$\mathrm{f}(\mathrm{Hz})$

Figure 4.8. Continued from last page.

\begin{tabular}{|c|c|c|c|}
\hline & Start Time (UT) & $\mathrm{U}_{10}(\mathrm{~m} / \mathrm{s})$ & $\theta_{\text {wind }}$ \\
\hline $\mathrm{m}$ & 1031 & 5.9 & $354^{\circ}$ \\
\hline $\mathrm{n}$ & 1103 & 6.7 & $355^{\circ}$ \\
\hline $\mathrm{o}$ & 1135 & 7.7 & $6^{\circ}$ \\
\hline $\mathrm{p}$ & 1207 & 7.0 & $9^{\circ}$ \\
\hline
\end{tabular}



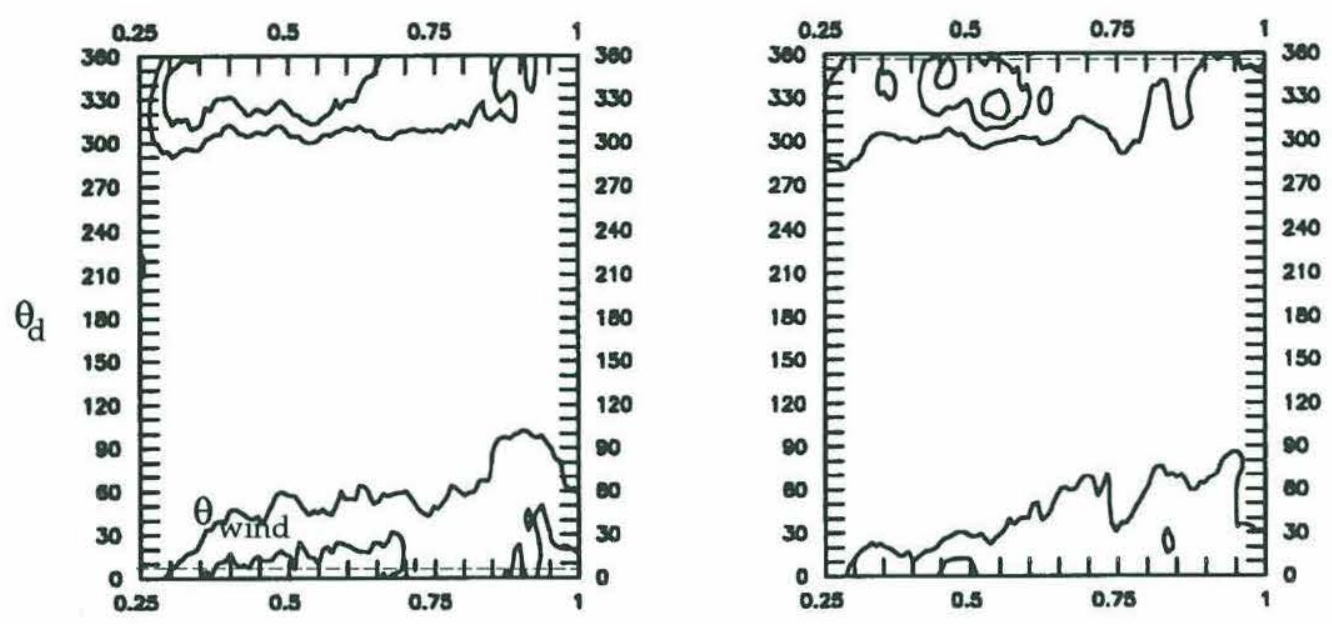

q
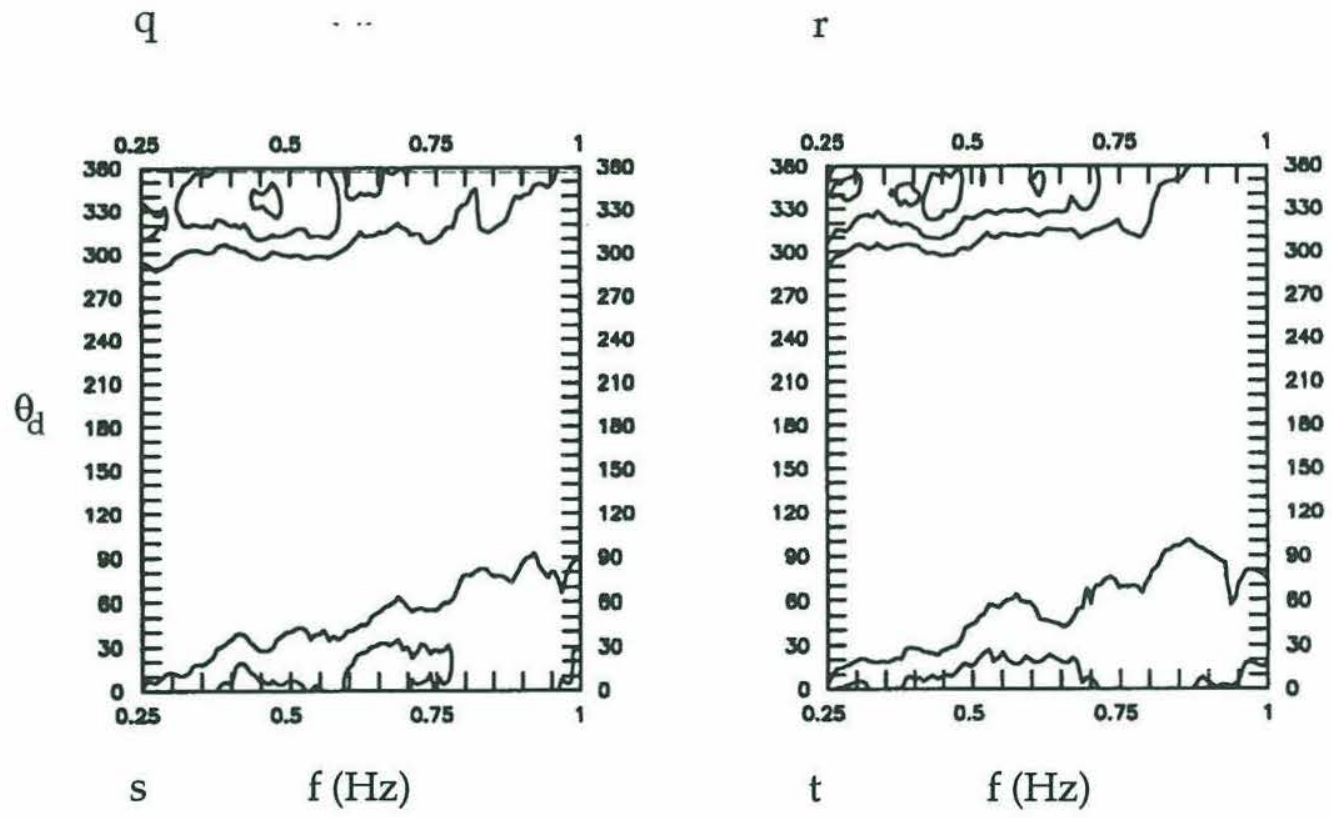

Figure 4.8. Continued from last page.

\begin{tabular}{|c|c|c|c|}
\hline & Start Time (UT) & $\mathrm{U}_{10}(\mathrm{~m} / \mathrm{s})$ & $\theta_{\text {wind }}$ \\
\hline $\mathrm{q}$ & 1239 & 7.0 & $3^{\circ}$ \\
\hline $\mathrm{r}$ & 1311 & 7.2 & $357^{\circ}$ \\
\hline $\mathrm{s}$ & 1343 & 7.9 & $358^{\circ}$ \\
\hline $\mathrm{t}$ & 1415 & 8.3 & $0^{\circ}$ \\
\hline
\end{tabular}


capacitance wire wave gauge ( Step 10 in Section 3.7.2 and Equation 3.19), we instead chose to normalize $\Phi(\mathrm{f}, \theta)$ such that

$$
\sum_{m=1}^{M} \Phi\left(f, \theta_{m}\right)=1 .
$$

Figure 4.8 shows contour plots of how the wave energy is distributed as a function of frequency subject to Equation 4.7. The response of the high frequency wave components to the change in wind direction is initiated in Figure 4.8i-1 when the wind speed decreases to approximately 4.0 meters/second. Prior to this, the wind has been blowing along $220^{\circ}$ for several days and the wave direction is correspondingly centered around that direction as well. In Figure $4.8 \mathrm{~m}$, we observe a region of high energy centered at $350^{\circ}$ for $\mathrm{f}>0.65 \mathrm{~Hz}$. Note that this is not observed in Figure $4.7 \mathrm{~m}$ because of the spectral energy distribution. The new wave direction matches the increasing wind speed at that direction $\left(354^{\circ}\right)$. The plot also suggests that the high frequency wave components respond quickly to changes in wind speed and wind direction. As the wind speed increases and time progresses, this high energy region grows and migrates towards the lower frequencies until the wave direction is aligned with the wind direction across all frequencies (Figure $4.8 \mathrm{r}-\mathrm{t}$ ).

\subsection{Discussion}

\subsubsection{Ambient noise, $u_{10}$ and $u_{*}$.}

Figure 4.9 shows plots of $\log \mathrm{U}_{10}$ versus the spectrum level at the different ambient noise frequencies $\mathrm{N}(\mathrm{f})$ given in Figure 4.6 showing a linear relationship between the 
Table 4.1. Some coefficients for the WOTAN expression and the equivalent power laws computed from NOBS data (c.f. Table 1.2 and Figure 4.9).

$$
\log \mathrm{U}_{10}=\mathrm{mN}+\mathrm{n} \quad \frac{\mathrm{p}^{2}}{\mathrm{p}_{\text {ref }}^{2}}=m \mathrm{U}_{10}^{n}
$$

\begin{tabular}{|c|c|c|c|c|c|}
\hline $\mathrm{f}(\mathrm{kHz})$ & $\mathrm{C}_{x y}$ & $\mathrm{~m}$ & $\mathrm{n}$ & $10^{-3} m$ & $n$ \\
\hline 4.3 & 0.94 & 0.0336 & -1.089 & 1.7 & 3.0 \\
\hline 8.0 & 0.93 & 0.0367 & -1.157 & 1.4 & 2.7 \\
\hline 14.0 & 0.78 & 0.0417 & -1.261 & 1.1 & 2.4 \\
\hline$\Sigma$ & 0.94 & 0.0376 & -2.057 & 296 & 2.7 \\
\hline
\end{tabular}

two parameters. We also plotted the linear regression equations from the different WOTAN measurements given in Table 1.2 and from our data. Regression lines in the form of the WOTAN equation (Equation 1.3) were computed from the data using an orthogonal least squares fit (Casella, 1990, p. 584) since both $\log U_{10}$ and $N(f)$ are random variables. A summary of the linear regression coefficients and the power law coefficients between the two parameters is given in Table 4.1. The equation of the regression lines for our data are comparable to those found by previous investigators. Our results suggest an ambient sound pressure level dependence

$$
\mathrm{p}^{2} \sim \mathrm{U}_{10}^{2.4-3.0}
$$

Figure 4.10 shows four plots of $\mathrm{N}$ versus $\log \mathrm{u}$ * sampled and computed from the wind speed measurements in this experiment. The data is well correlated. It suggests that a single linear fit to the data is adequate and that the power law expression 

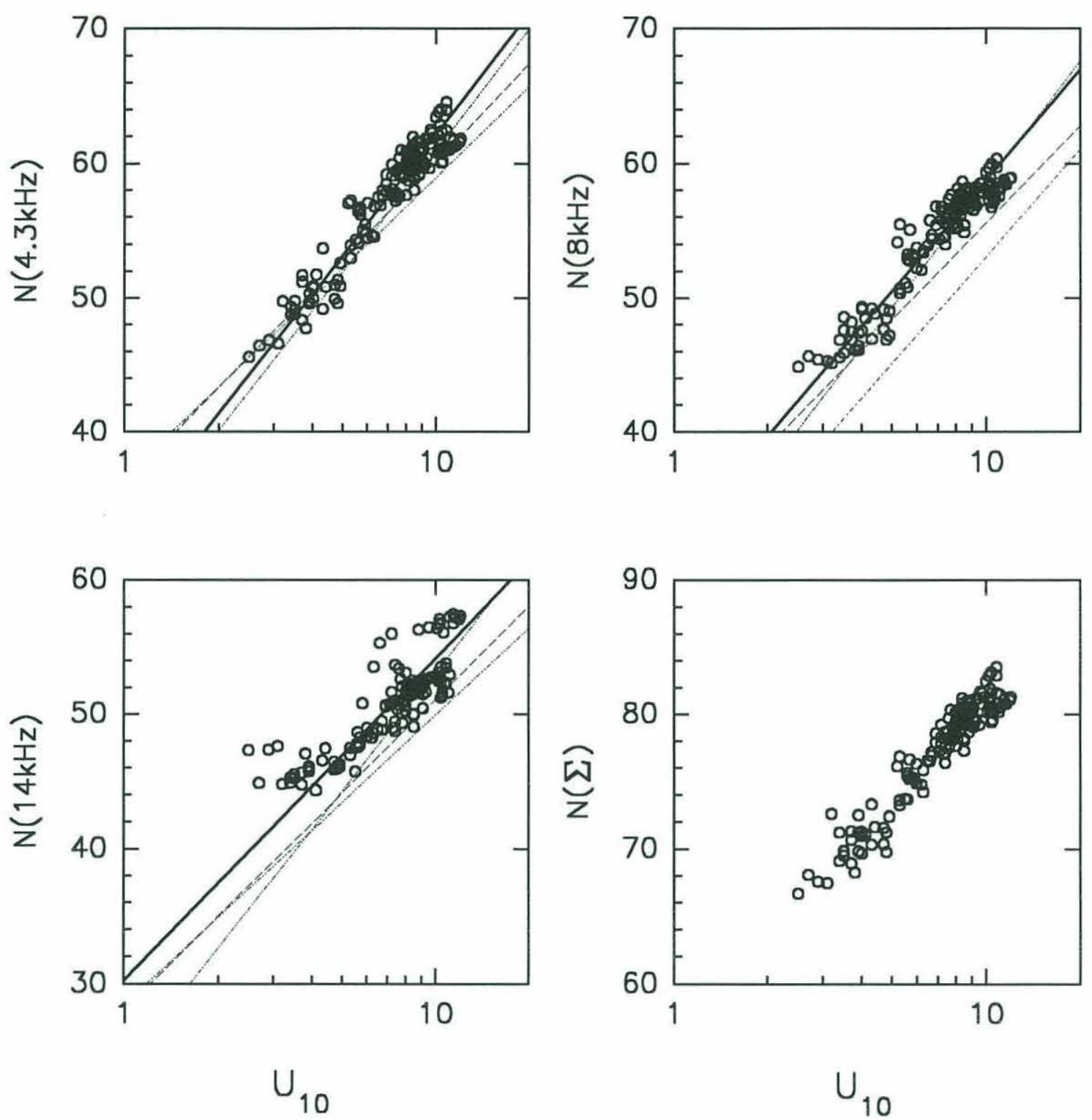

Figure 4.9. Plot of $U_{10}$ vs. selected $N$ frequencies. Lines indicate regression lines obtained by various investigations. This study - solid line, Evans et al (1984) - short dash, Lemon et al (1984) - dash-dot-dot, Vagle et al (1990) - dash-dot. 

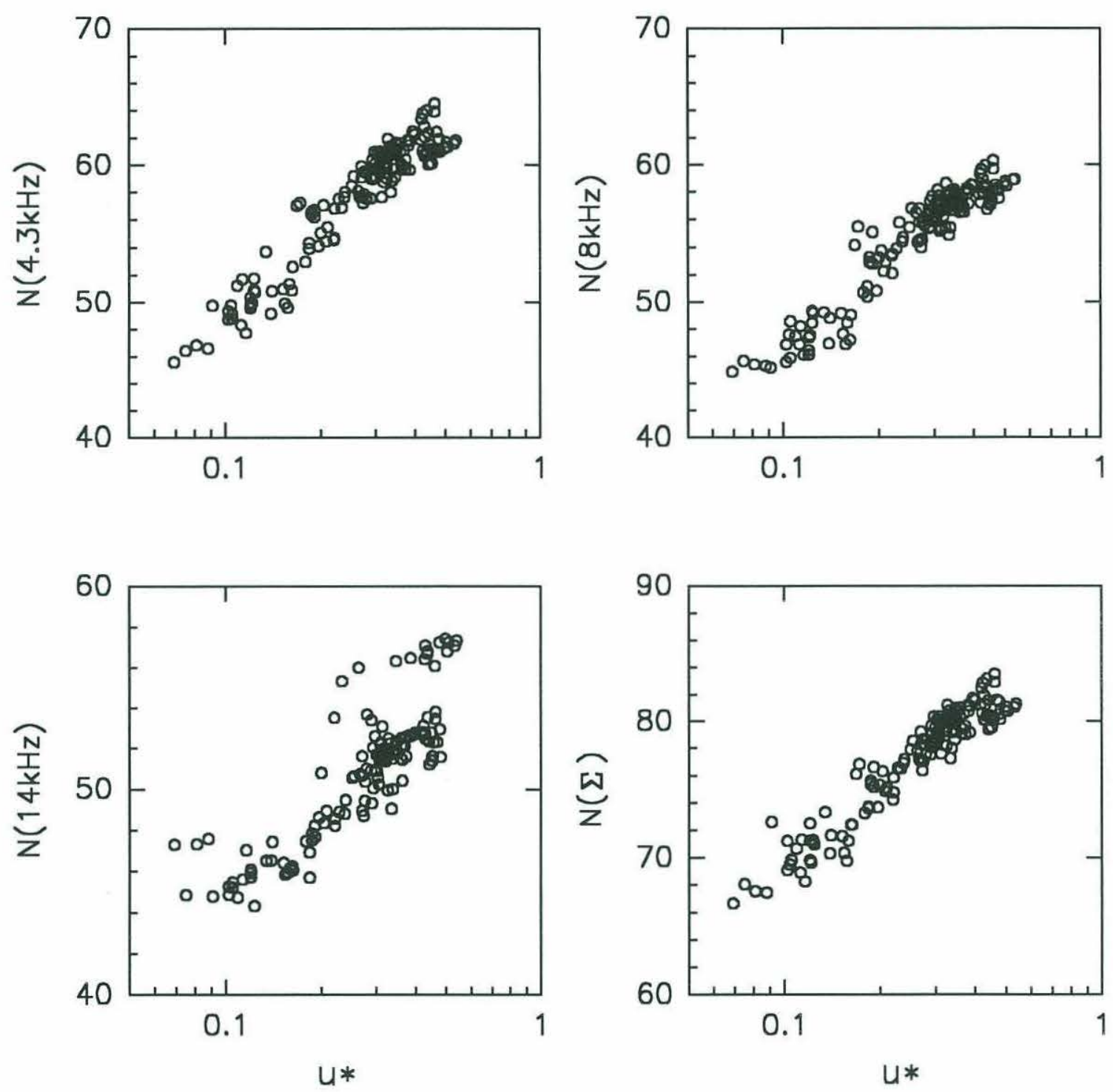

Figure 4.10. Plot of $u_{*} v$ s. selected $\mathrm{N}$ frequencies. The figures show a good correlation between $\mathrm{u} *$ and $\mathrm{N}$. 

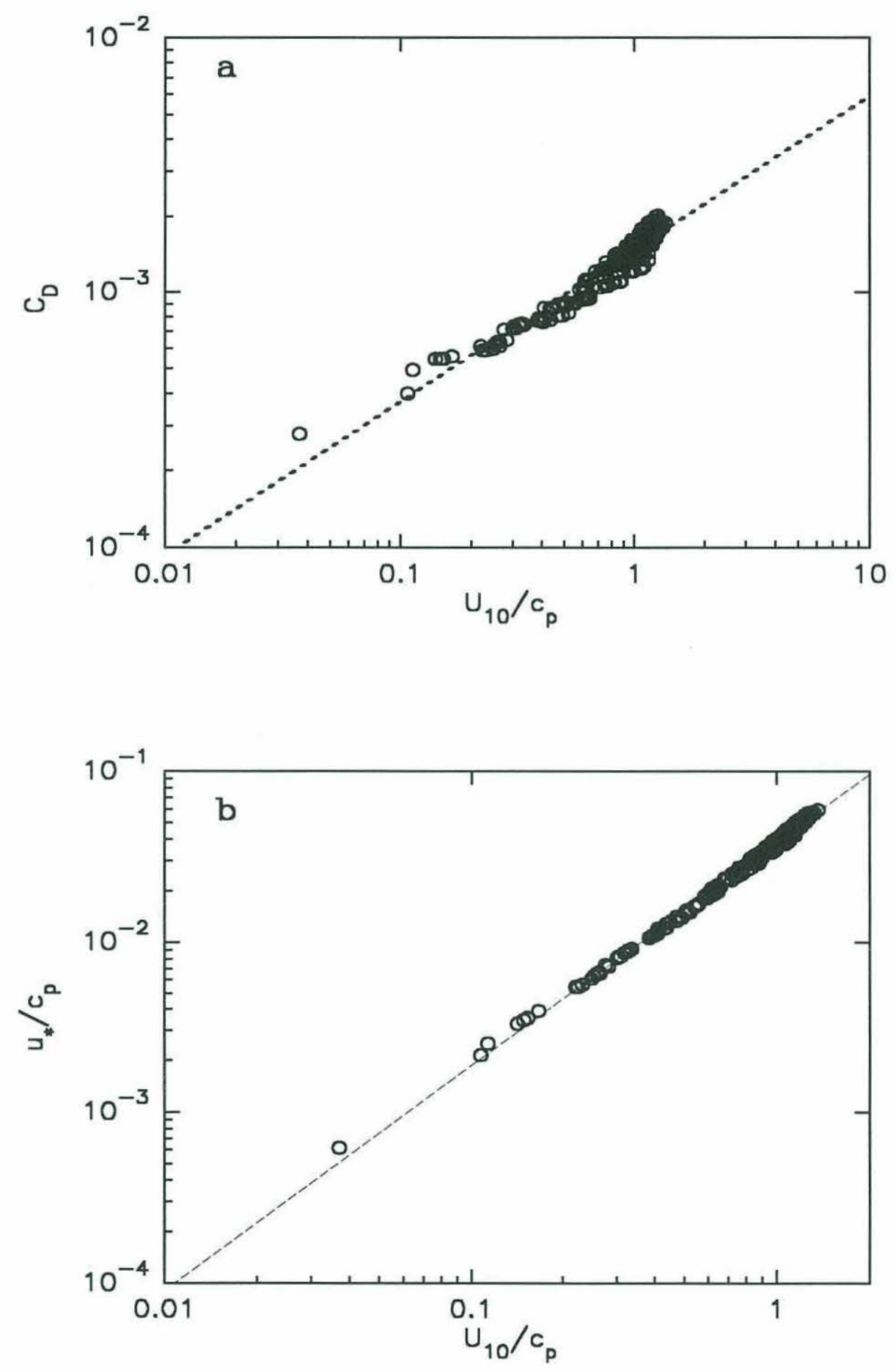

Figure 4.11. a) Plot of the drag coefficient $C_{D}=\left(u_{*} / U_{10}\right)^{2}$ as a function of $\left(U_{10} / c_{p}\right)$. b) Plot relating the inverse wave age based on $\mathrm{U}_{10}$ and on $\mathrm{u}_{*}$. 
Table 4.2. Linear regression coefficients relating $\mathbf{u}_{*}$ and $\mathbf{N}$ and the equivalent power laws computed from NOBS data (c.f. Figure 4.10).

$$
\log \mathrm{u}_{*}=\mathrm{mN}+\mathrm{n} \quad \frac{\mathrm{p}^{2}}{\mathrm{p}_{\text {ref }}^{2}}=m \mathrm{u}_{*}^{n}
$$

\begin{tabular}{|c|c|c|c|c|c|}
\hline $\mathrm{f}(\mathrm{kHz})$ & $C_{x y}$ & $\mathrm{~m}$ & $\mathrm{n}$ & $10^{-6} m$ & $n$ \\
\hline 4.3 & 0.95 & 0.0443 & -3.138 & 12.1 & 2.3 \\
\hline 8.0 & 0.95 & 0.0485 & -3.228 & 4.5 & 2.1 \\
\hline 14.0 & 0.86 & 0.0554 & -3.384 & 1.3 & 1.8 \\
\hline$\Sigma$ & 0.95 & 0.0496 & -4.420 & 815 & 2.0 \\
\hline
\end{tabular}

$$
\mathrm{p}^{2} \sim \mathrm{u}_{*}^{1.8-2.3}
$$

best describes the data (see Table 4.2). Our results contradict Kerman (1984) who argued based on empirical data that $\mathrm{p}^{2} \sim \mathrm{u}_{*}^{1.5}$ when $\mathrm{u}_{*}>\mathrm{u}_{*_{\mathrm{C}}}=0.23 \mathrm{~m} / \mathrm{s}$, and $\mathrm{p}^{2} \sim \mathrm{u}_{*}{ }^{3}$

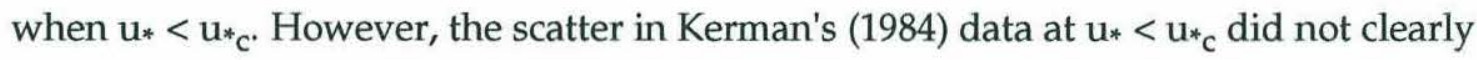
show that there are indeed two subranges.

Given that $\mathrm{u}_{*}$ was computed primarily from $\mathrm{U}_{\mathrm{z}}$ in both Kerman (1984) and in this work (Equations 4.2 and 4.3) and is only weakly dependent on $c_{p}$, the relationship between $\mathrm{u} *$ and $\mathrm{N}$ is essentially similar to that between $\mathrm{N}$ and $\mathrm{U}_{10}$.

In Section 4.1, we described an implicit method using Maat et al's (1991) equations for the relationship between the value of $\mathrm{u}_{*}, \mathrm{U}_{\mathrm{z}}$ and the wave age. It is desirable to obtain an explicit and dimensionally consistent expression for $\mathrm{u}_{*}$ as a function of $\mathrm{U}_{10}$ that is equivalent to their method. This can easily be done from our results. 
Figure 4.11a shows a log-log plot of the drag coefficient $C_{D}=\left(u_{*} / U_{10}\right)^{2}$ versus the inverse wave age $\left(U_{10} / c_{p}\right)$ showing an increase of the sea surface roughness with increasing wind. In this figure we see that the relationship between the two variables can be approximated by a power law

$$
\mathrm{C}_{\mathrm{D}}=1.48 \times 10^{-3}\left(\frac{\mathrm{U}_{10}}{\mathrm{c}_{\mathrm{p}}}\right)^{0.6}
$$

By rearranging the variables in Equation 4.10, we can obtain an expression relating $\mathrm{U}_{10}, \mathrm{u} *$ and $\mathrm{c}_{\mathrm{p}}$. Figure $4.11 \mathrm{~b}$ plots the two versions of the inverse wave age expression, $\left(\mathrm{U}_{10} / \mathrm{c}_{\mathrm{p}}\right)$ and $\left(\mathrm{u}_{*} / \mathrm{c}_{\mathrm{p}}\right)$, against each other. The regression line on the plot translates to a power law expression

$$
\begin{aligned}
\left(\frac{u_{*}}{c_{p}}\right) & =3.85 \times 10^{-2}\left(\frac{U_{10}}{c_{p}}\right)^{1.3}, \\
u_{n} & =3.85 \times 10^{-2} U_{10}^{1.3} c_{p}^{-0.3} .
\end{aligned}
$$

\subsubsection{Ambient noise and wave parameters.}

The previous field experiments reviewed in Section 1.2 [Penhallow \& Dietz (1964), Perrone (1969), Lemon \& Farmer (1984)] examined the relationship between ambient noise and the significant wave height (or alternatively, the root mean square amplitude $a$ ) and found that these two parameters poorly correlated. The plots in Figure 4.12 which compares our measurements of $\mathrm{N}$ and $a$ is consistent with their 
results. We suggested that the presence of acoustically inactive swell affects the relationship between $\mathrm{N}$ and $a$. While the presence of swell significantly increases $a$, it does not proportionally increase the steepness of the waves nor does it proportionally increase the incidence of breaking.

To reduce the influence of the swell energy on the variance of the wave field, we filtered the swell out of the wave height record and computed the root mean square amplitude of the data (see Section 4.3). The resulting $a_{w}$ estimate, whose time series is plotted in Figure 4.3c, was plotted against N (Figure 4.13). In this figure, we see that although the scatter in the data is greater than that between $U_{10}$ and $N$, the improvement in the correlation between $a$ and $\mathrm{N}$ is significant. Table 4.3 shows that the power law relationship is given by

$$
\mathrm{p}^{2} \sim a_{w}^{1.8-2.2}
$$

We can better understand the relationship between the wind speed and wind waves by examining the behavior of the high frequency wave components. In Figure 4.4, we

Table 4.3. Linear regression coefficients relating $a_{w}$ and $\mathrm{N}$, and the equivalent power laws computed from NOBS data.

$$
\log a_{w}=\mathrm{mN}+\mathrm{n} \quad \mathrm{p}^{2}=m a_{w}^{n}
$$

\begin{tabular}{|c|c|c|c|c|c|}
\hline $\mathrm{f}(\mathrm{kHz})$ & $\mathrm{C} x y$ & $\mathrm{~m}$ & $\mathrm{n}$ & $10^{-6} m$ & $n$ \\
\hline 4.3 & 0.89 & 0.0450 & -3.126 & 8.8 & 2.2 \\
\hline 8.0 & 0.89 & 0.0496 & -3.236 & 3.3 & 2.0 \\
\hline 14.0 & 0.77 & 0.0547 & -3.297 & 1.1 & 1.8 \\
\hline$\Sigma$ & 0.87 & 0.0494 & -4.348 & 633 & 2.0 \\
\hline
\end{tabular}




\section{Chapter 4}

showed the time series of the wave spectrum $\Phi(\mathrm{f})$ for $\mathrm{f}=0.25,0.5 .0 .75$, and $1.0 \mathrm{~Hz}$. We noted that the $\Phi(f)$ time series closely match the shape of both the $U_{10}$ and $N$ time series. The behavior of the $\Phi(\mathrm{f})$ resembles $\mathrm{U}_{10}$ and $\mathrm{N}$ better than the $a_{w}$ time series.

Since the wind wave mean frequency $\overline{\mathrm{f}}$ is between 0.15 and $0.4 \mathrm{~Hz}$ (Figure $4.2 \mathrm{~d}$ ), then frequencies greater than $0.4 \mathrm{~Hz}$ are within the wind wave frequency range.

In the last section, we observed from the directional wave spectrum plots (Figure 4.7 and 4.8 ) that the higher frequency wind wave components ( $\mathrm{f}>0.5 \mathrm{~Hz}$ ) respond quickly to changes in wind speed and direction. Figure 4.14 shows plots of $\log \Phi$ at $0.5,0.75$, and $1.0 \mathrm{~Hz}$ versus $\mathrm{N}$. They show a well correlated relationship between these frequencies and $\mathrm{N}$ except the lower $\Phi(0.5 \mathrm{~Hz})$ values (low wind speed, $\Phi(0.5 \mathrm{~Hz})<-1.0)$ where the scatter and nonlinearity in the data is more considerable. However, the scatter in rest of the $\Phi(0.5 \mathrm{~Hz})$ data is considerably smaller.

Our results show that $a$ and $\mathrm{N}$ are poorly correlated partly because of the presence of swell. The characteristics of swell are not directly determined by the local wind-wave relationship and its influence on the local noise generation mechanisms is weak. By neglecting swell and concentrating on the wind sea portion of the wave spectrum, we can obtain a better correlated power law relationship between $\mathrm{N}$ and $a$. This is clearly shown by improved correlation between the energy at the higher frequency components of $\Phi$ and N (Figure 4.14).

Experiments by Rapp \& Melville (1991) and Loewen \& Melville (1991a) have suggested that the wave slope of a packet of $M$ waves 

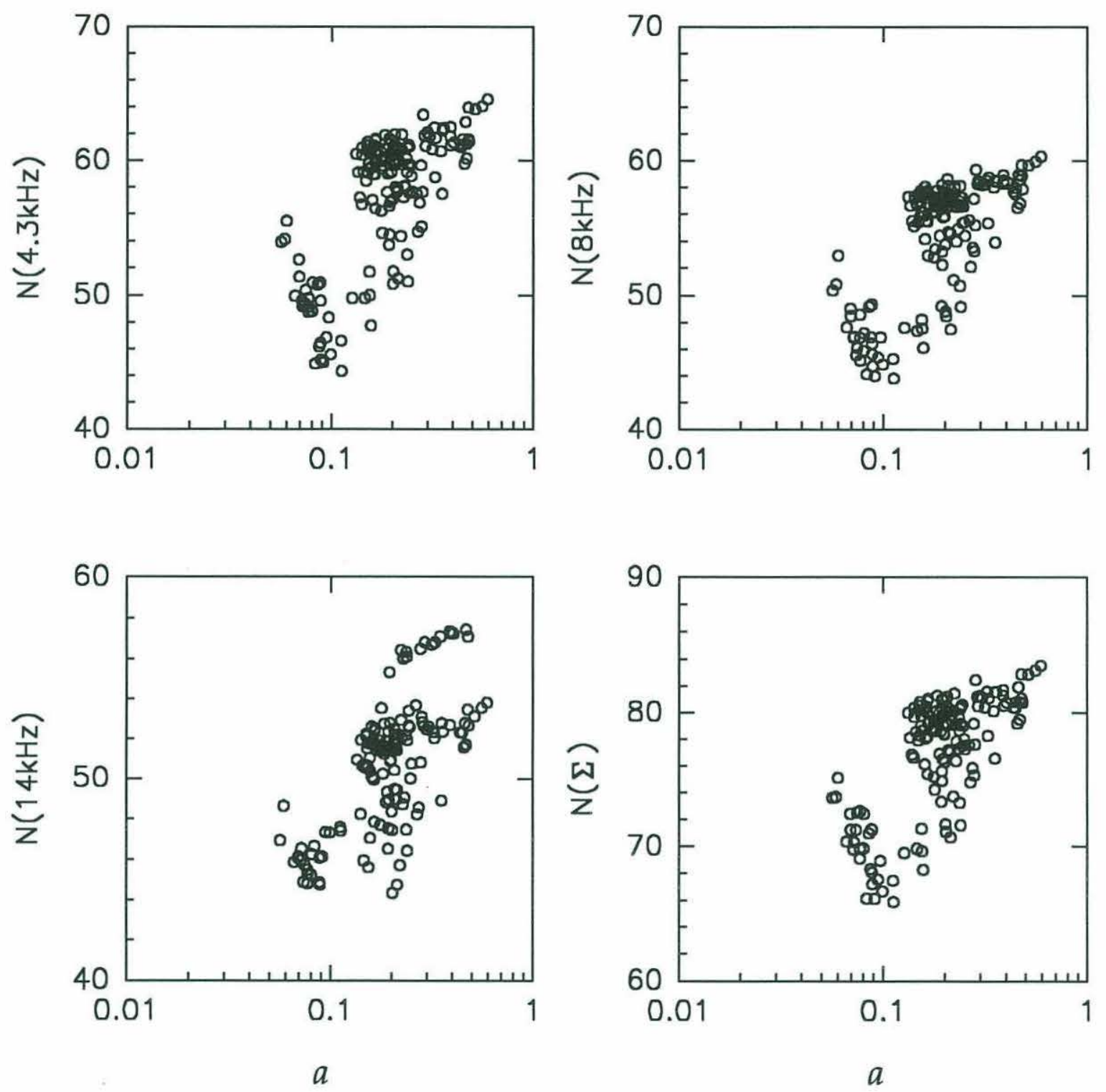

Figure 4.12. Plot of $\mathrm{N}$ vs. the root mean square wave amplitude $a$. The scatter in the data give correlation coefficients $=0.67$ for all the plots. 

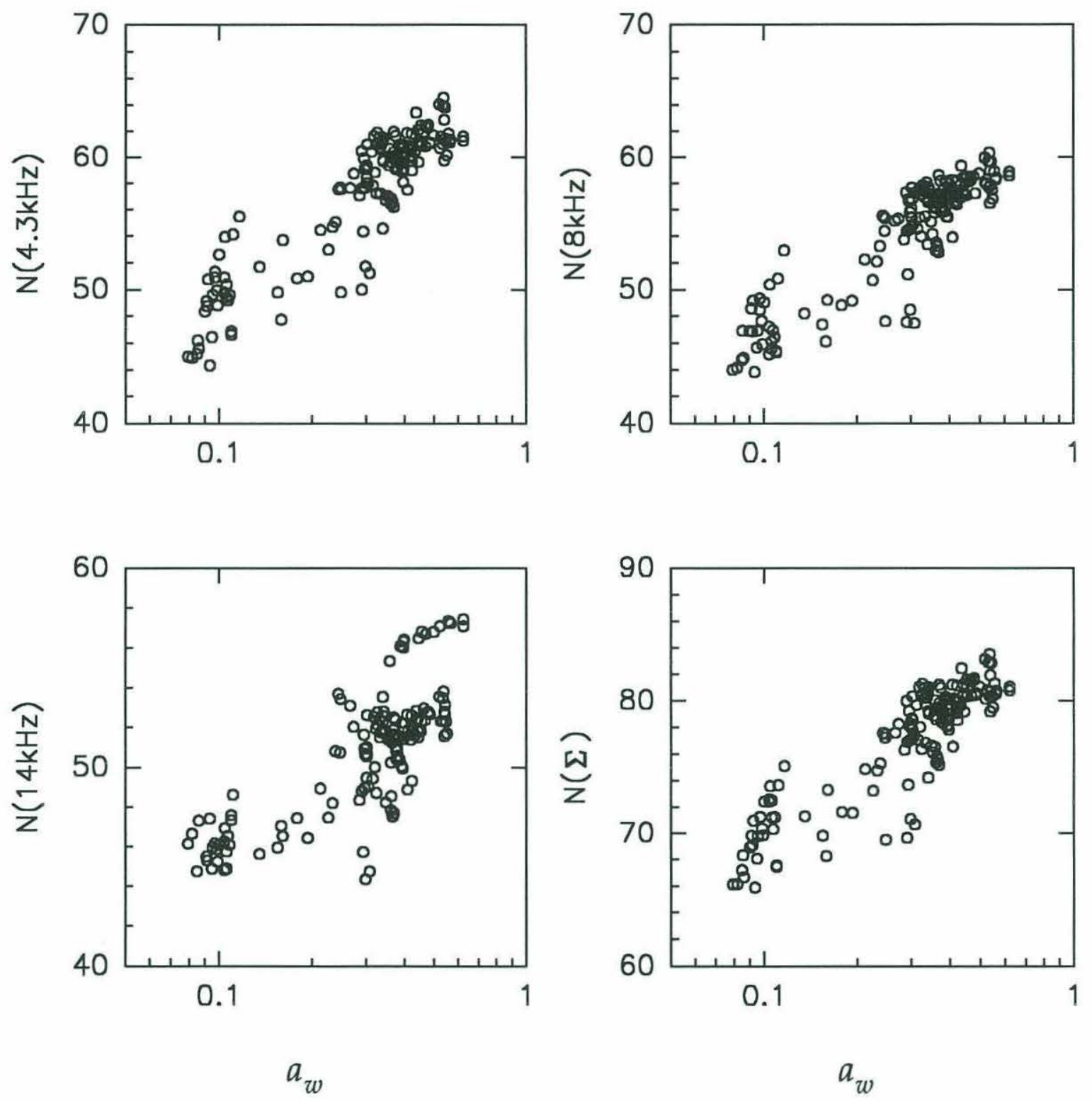

Figure 4.13. Plot of $\mathrm{N}$ vs. $a_{w}$. The surface elevation time series was high pass filtered to eliminate the contribution of swell energy to $a$. The figure shows that compared to Figure 4.12, we see a significant improvement in the correlation between ambient noise $\mathrm{N}$ and the root mean square wind wave amplitude $a_{w}$ (see Table 4.3). 

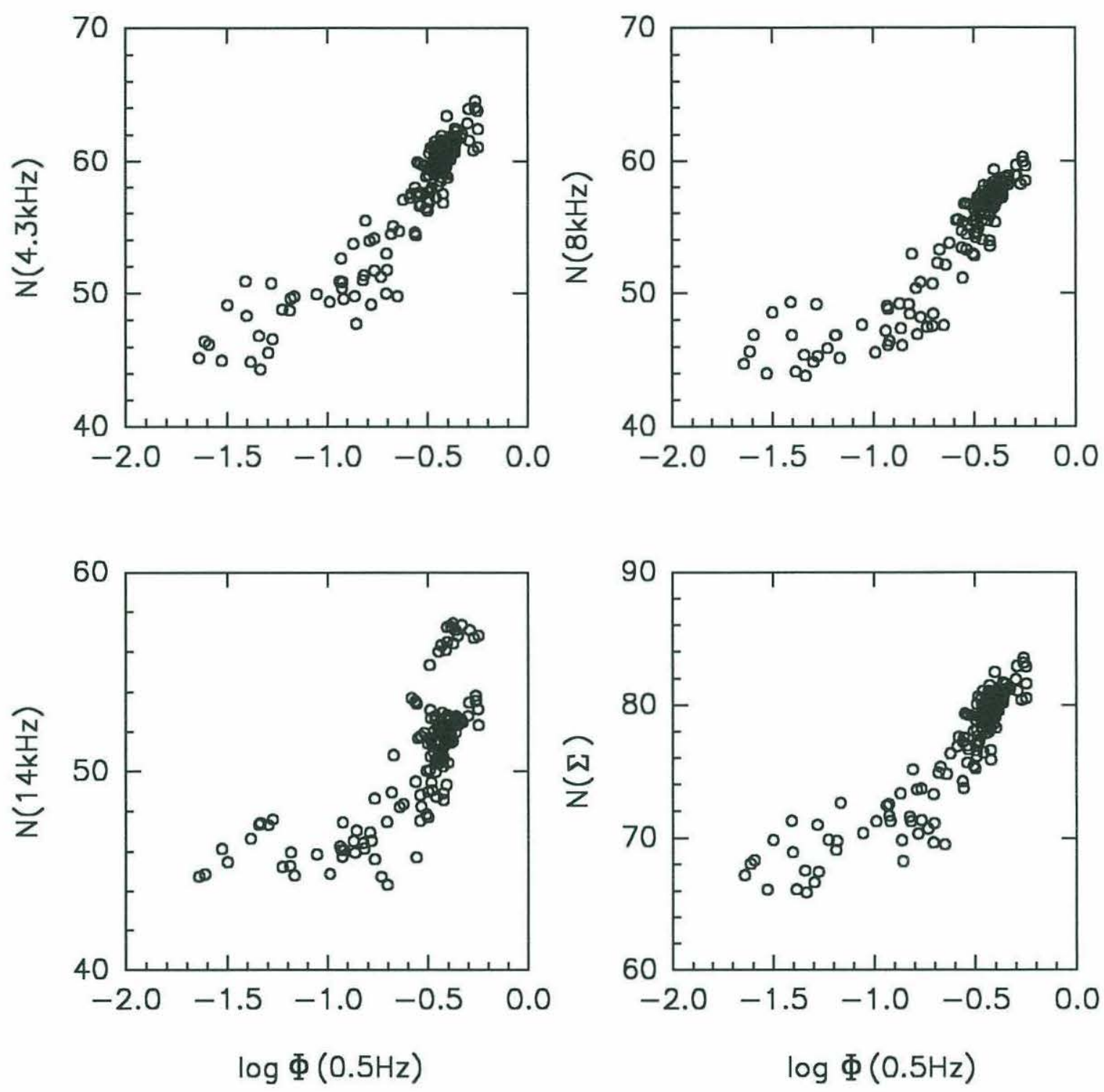

Figure 4.14. The high frequency surface wave energy and ambient noise $\mathrm{N}$ are wellcorrelated (see Table 4.5). a) Plot of N vs. $\Phi(0.5 \mathrm{~Hz})$. 

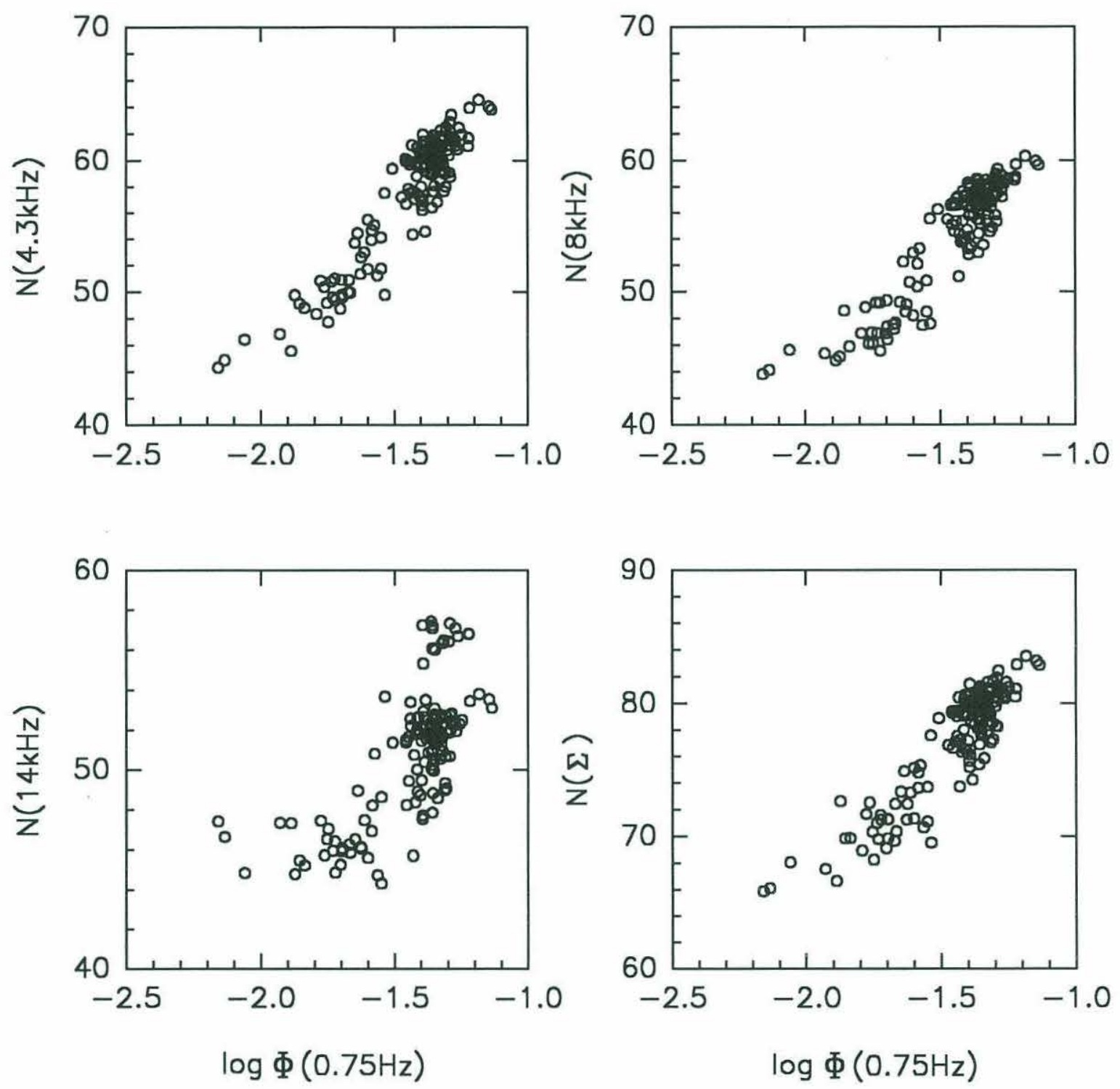

Figure 4.14. b) Plot of $\mathrm{N}$ vs. $\Phi(0.75 \mathrm{~Hz})$. 

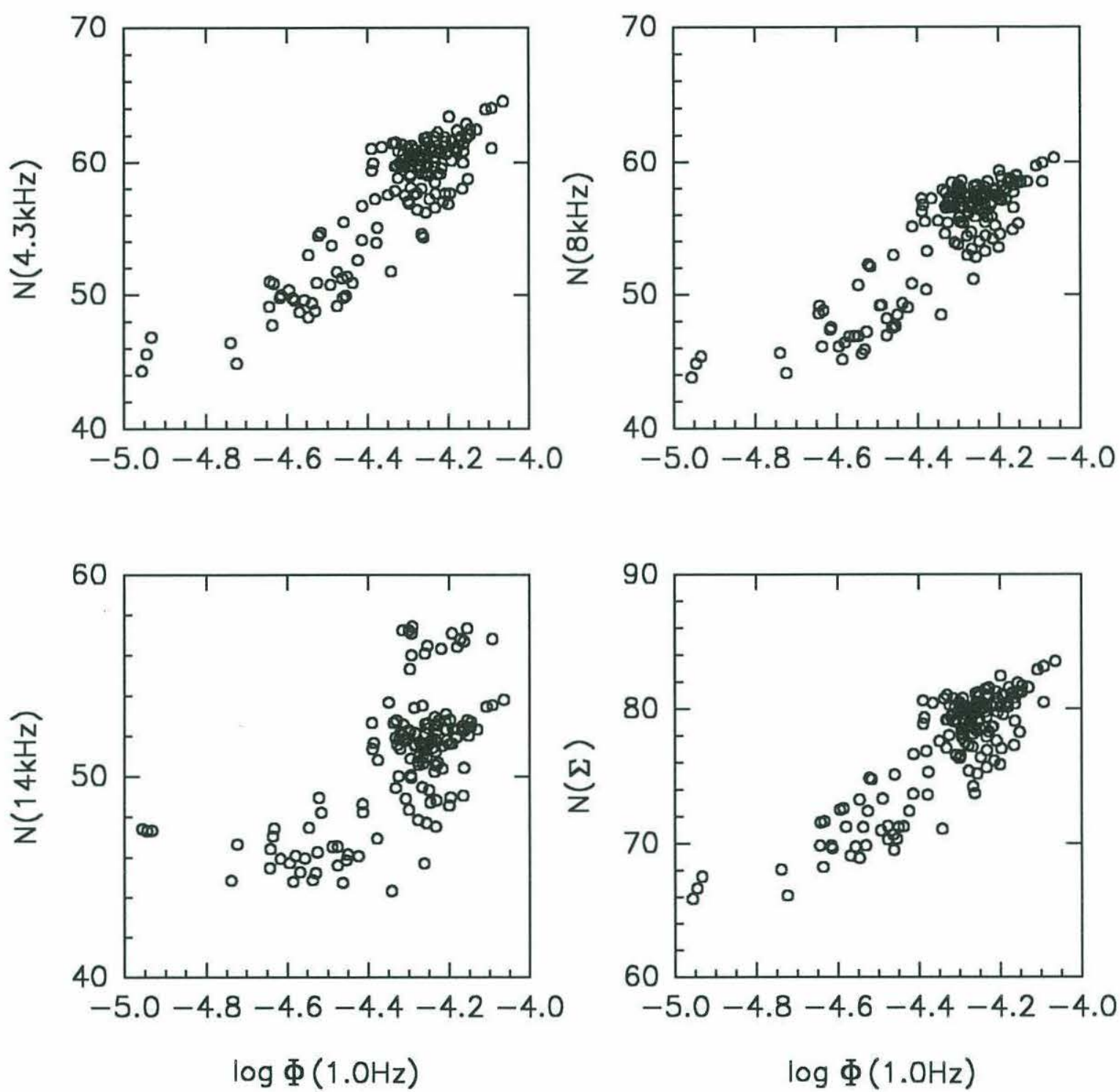

Figure 4.14. c) Plot of N vs. $\Phi(1.0 \mathrm{~Hz})$. 


$$
s_{p}=\sum_{i=1}^{M} a_{i} k_{i}
$$

can be used to measure the strength of wave breaking in the laboratory. Their experiments also suggest that $\mathrm{s}_{\mathrm{p}}$ correlates with several important dynamical properties of wave breaking, e.g., loss of excess momentum flux, mixing, dissipation, noise generation. Their results suggest that the overall steepness of the wave field is an important indicator of the characteristics of the breaking wave. We can show that the steepness of the wave field correlates with some dynamic characteristics of wave breaking in the ocean as well.

The time series of the RMS wave slope $s$ was given in Figure 4.5. A comparison of this plot with those of the $U_{10}$ and $N$ time series (Figures 4.1 and 4.5) shows that the general characteristics of those plots can also be observed here. In Figure 4.17 we presents four plots of $\log s$ versus $N$. The plots show that, consistent with the laboratory results of Melville et al (1988) and Loewen \& Melville (1991a), the steepness of the wave field correlates well with the ambient noise. The characteristics of this relationship are summarized in Table 4.4 which gives correlation coefficients

$$
C_{x y}=\frac{\sum_{i=1}^{N}\left(x_{i}-\bar{x}\right)\left(y_{i}-\bar{y}\right)}{\sqrt{\sum_{i=1}^{N}\left(x_{i}-\bar{x}\right)^{2}} \sqrt{\sum_{i=1}^{N}\left(y_{i}-\bar{y}\right)^{2}}}
$$



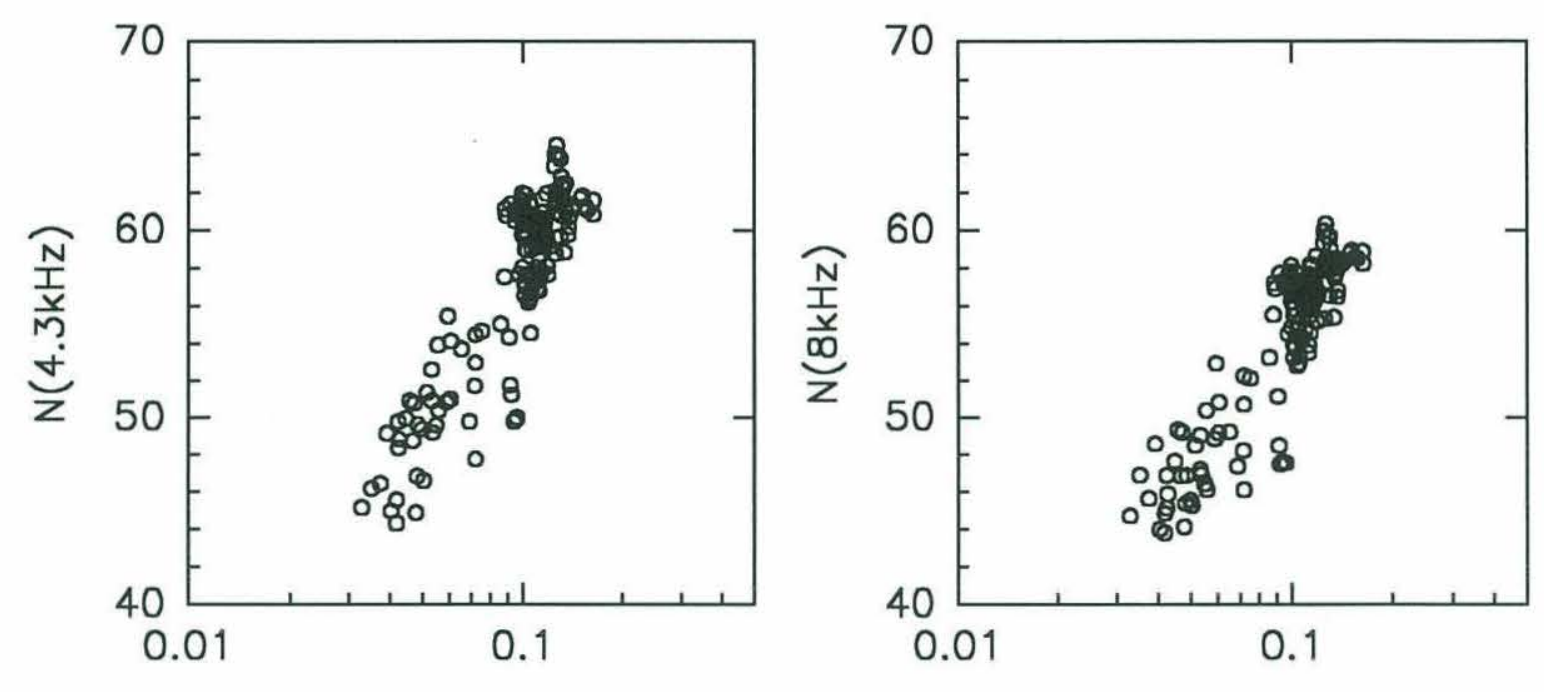

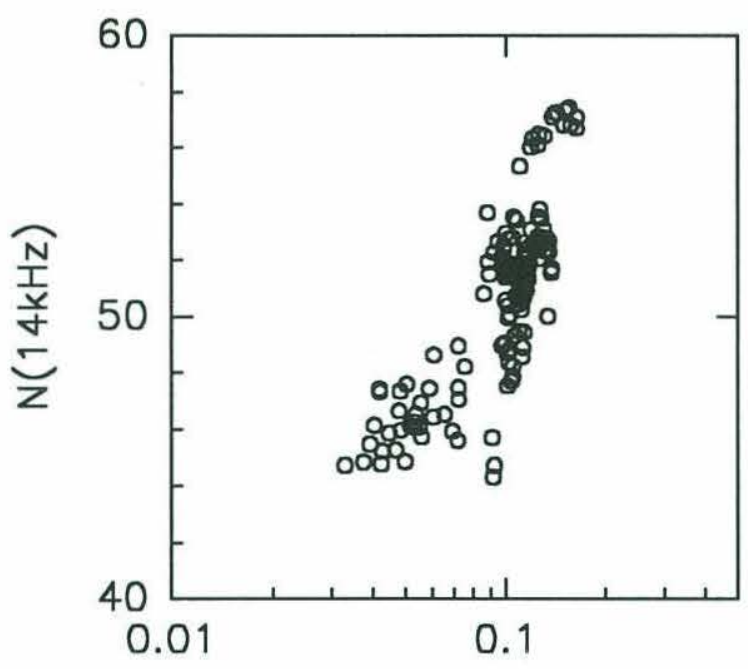

$S$

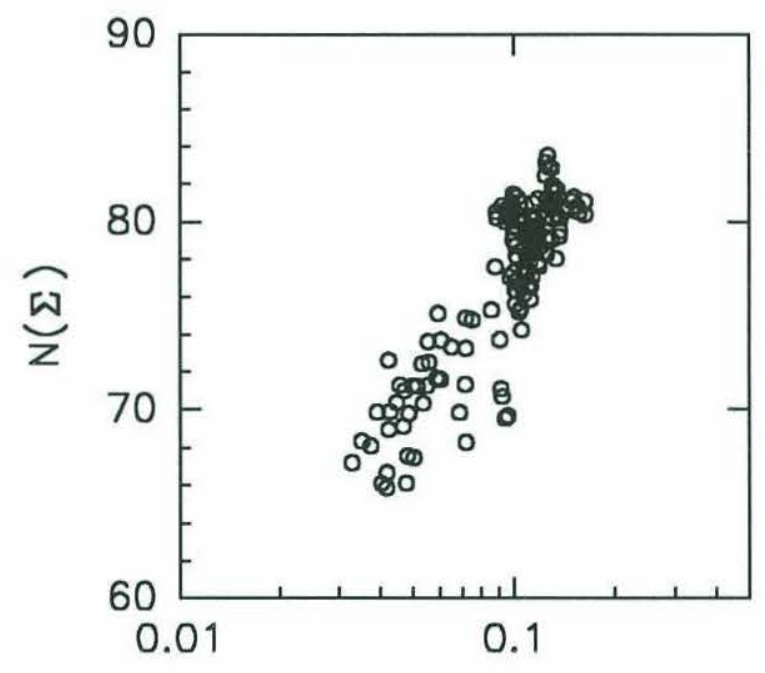

$S$

Figure 4.15. Plot of wave slope $s$ versus the ambient noise $\mathrm{N}$ at selected frequencies. 

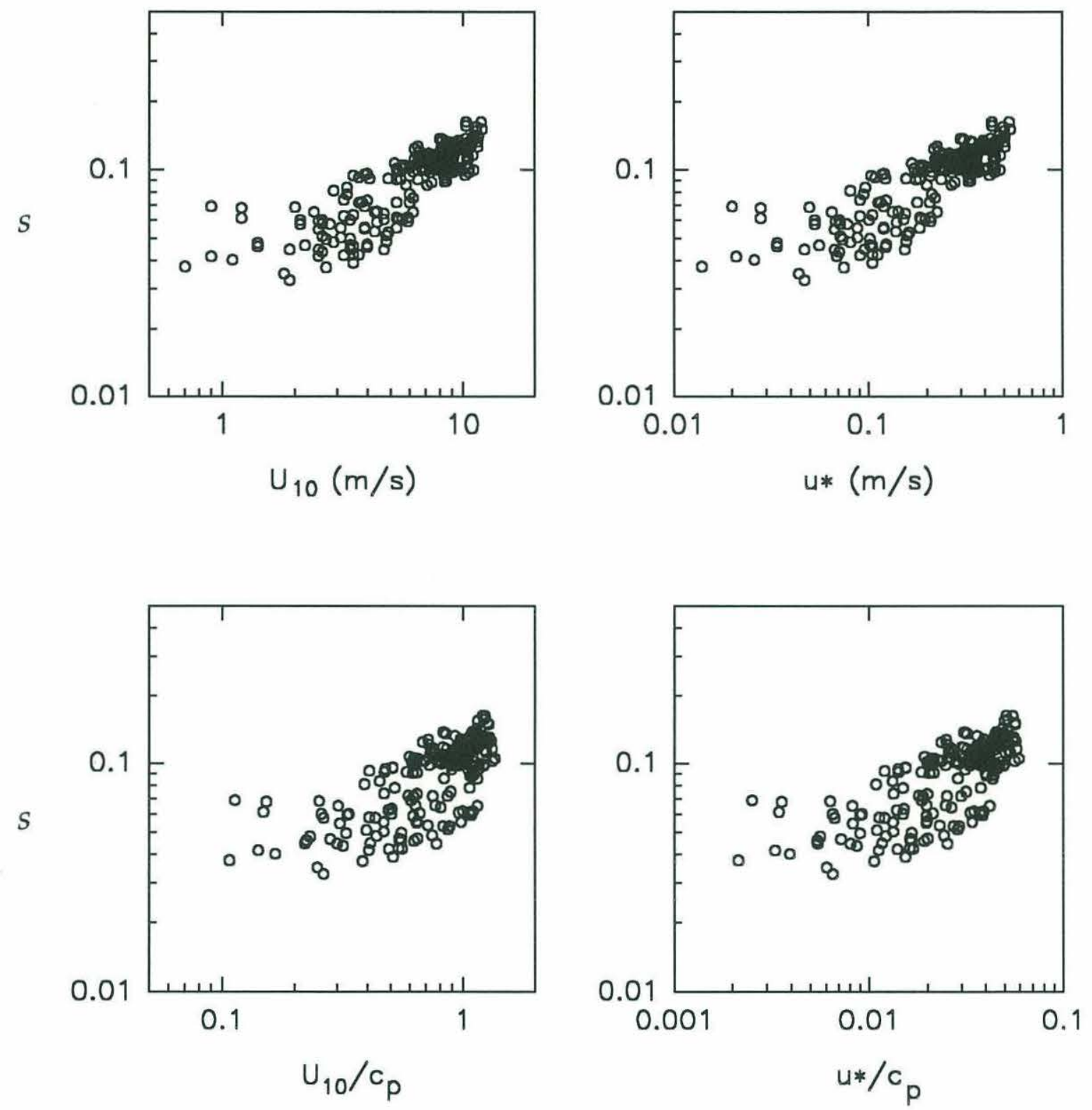

Figure 4.16. Plot of wave slope $s$ as a function of wind speed $U_{10}$, wind stress $u_{*}$ and inverse wave age $\left(\mathrm{U}_{10} / \mathrm{c}_{\mathrm{p}}\right)$ and $\left(\mathrm{u}_{*} / \mathrm{c}_{\mathrm{p}}\right)$. 
Table 4.4. Power law coefficients for the ambient sound pressure level and wave slope $s$, and the corresponding correlation coefficients $C_{x y}$ between $\mathrm{N}$ and $\log s$.

$$
\frac{\mathrm{p}^{2}}{\mathrm{p}_{\mathrm{ref}}^{2}}=m s^{n}
$$

\begin{tabular}{|c|c|c|c|}
\hline $\mathrm{f}(\mathrm{kHz})$ & $10^{-8} m$ & $n$ & $C_{x y}$ \\
\hline 4.3 & 18.5 & 3.4 & 0.87 \\
\hline 8.0 & 2.9 & 3.1 & 0.88 \\
\hline 14.0 & 0.23 & 2.6 & 0.81 \\
\hline$\Sigma$ & 802 & 3.1 & 0.85 \\
\hline
\end{tabular}

greater than 0.85 between $\mathrm{N}$ and $\log s$ except for the $14 \mathrm{kHz}$ noise. The plots of wave slope versus $\mathrm{N}$ give a power law relationship

$$
\mathrm{p}^{2} \sim s^{3.1-3.4}
$$

We excluded the $\mathrm{N}(14 \mathrm{kHz})$ regression because of the significantly poorer correlation coefficient with this ambient noise frequency. As we mentioned in Section 1.1, field experiments [Farmer \& Lemon (1984), Vagle et al (1990)] have suggested that the influence of sound absorption by bubbles can significantly alter the ambient noise levels above $8 \mathrm{kHz}$.

Figure 4.16 shows four plots relating $s$ to $\mathrm{U}_{10}, \mathrm{u} *$ and wave age parameters based on both $\mathrm{U}_{10}$ and $\mathrm{u}_{*}$. While most of the data points in the figure are well correlated, the 
scatter in the data is more significant than in Figure 4.15. The scatter for data points for $U_{10}<5.5 \mathrm{~m} / \mathrm{s}\left[\mathrm{u}^{*}<0.2 \mathrm{~m} / \mathrm{s}\right]$ appears to be greater than for data points above it.

\subsubsection{Ambient noise and wind-wave development.}

In making simultaneous wind, wave and ambient noise measurements, Penhallow \& Dietz (1964) subdivided their data into portions where the wind was steady, increasing and decreasing. Their results show (see Section 1.2) that the linear correlation coefficient between the ambient noise at $630 \mathrm{~Hz}$ and the wind and wave variables is higher when the wind is steady and degrades considerably when the wind is changing. They theorized that when the winds are steady and the waves are fully developed, there is an equilibrium between the rate of energy input by the wind to the waves and the rate of energy dissipated by waves due to wave breaking. It can then be reasoned that since the wind and ambient noise relationship is well-defined and well-correlated, then the wave conditions that satisfy this equilibrium should be well-correlated with ambient noise as well. Unfortunately, there are no plots or figures in their paper with which we can examine the data nor was their research followed up in the literature. As we mentioned in our review, the research in wave and ambient noise relationship is relatively sparse.

We decided to further explore the ideas in Penhallow \& Dietz (1964) by subdividing the available NOBS data and performing a similar comparison. While the boundary between the different sections of the data were determined by inspection, they were chosen on the basis of predetermined and well-defined characteristics of the data. Based on an examination of the NOBS data, the time series was subdivided into four general conditions (Figure 4.17), 
Table 4.5. Linear correlation coefficients between $\mathrm{N}(4.3 \mathrm{kHz})$, and the log of several wind and wave parameters.

\begin{tabular}{|c|c|c|c|c|c|c|}
\hline & \multicolumn{3}{|c|}{$\mathrm{N}(4.3 \mathrm{kHz})$} & \multicolumn{3}{c|}{$\log \mathrm{U}_{10}$} \\
\hline $\log ($ param$)$ & all data & steady & unsteady & all data & steady & unsteady \\
\hline $\mathrm{U}_{10}$ & 0.94 & 0.84 & 0.96 & 1.00 & 1.00 & 1.00 \\
\hline$a$ & 0.66 & 0.61 & 0.69 & 0.70 & 0.53 & 0.75 \\
\hline$a_{w}$ & 0.87 & 0.61 & 0.84 & 0.87 & 0.59 & 0.90 \\
\hline$\Phi(0.50)$ & 0.92 & 0.78 & 0.91 & 0.86 & 0.66 & 0.87 \\
\hline$\Phi(0.75)$ & 0.90 & 0.62 & 0.89 & 0.82 & 0.47 & 0.83 \\
\hline$\Phi(1.00)$ & 0.87 & 0.54 & 0.86 & 0.79 & 0.38 & 0.80 \\
\hline
\end{tabular}

I. Decaying and growing wind speed and wave amplitude.

II. Fully developed quasi-steady wind and wave condition.

III. Turning winds.

IV. Decaying wind and wave condition.

For our purposes, we considered portion II of the record as steady, and portions I, III and IV unsteady. We then computed the correlation coefficients for the steady, unsteady and the full time series records for the wind and wave variables, and $\mathrm{N}(4.3 \mathrm{kHz})$. The results are summarized in Table 4.5 .

Unlike Penhallow \& Dietz, our results show that the correlation coefficients for unsteady data is higher than those for steady data. This is observed in both the wind and wave data. The difference between the correlation coefficient of the full data set 

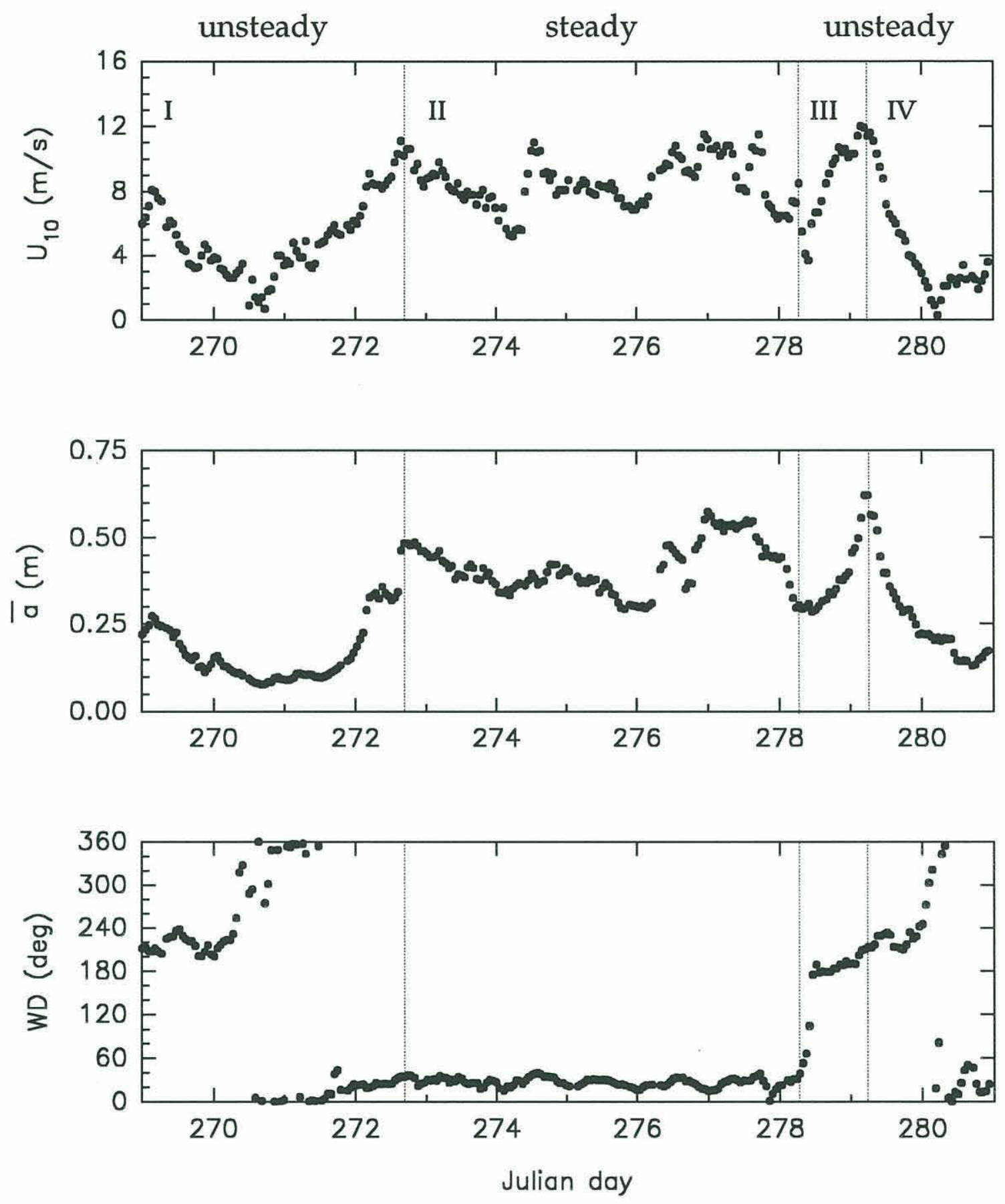

Figure 4.17. The wind and wave time series during NOBS can be subdivided into steady and unsteady conditions. The data in section II were considered steady while those in sections I, III and IV were considered unsteady. 
and the unsteady data is negligible. The reduction in the correlation coefficient during steady conditions is more significant for wave parameters.

The plots in Figures 4.18 and 4.19 show that although the scatter in the data is comparable for the steady and unsteady cases, the range of the unsteady data is bigger and it is comparable to the full data range. Consequently, the correlation coefficient of the unsteady data is comparable to the full data and is significantly larger than that of the steady data. It is not clear why the Penhallow \& Dietz (1964) results are different from ours since they did not provide enough details in their paper for us to examine their data. However, given the closeness of the correlation coefficients between the unsteady and full cases, we believe our results are robust and the conclusions in this work are not sensitive to the quantity of the data we analyzed.

\subsubsection{Wave growth and wave age.}

We can relate the wind speed $U_{10}$ to the wave energy by adopting Kitaigorodskii's (1962) non-dimensionalization of the wave and wind variables. This approach, which was used by Hasselmann et al (1973) to analyze fetch-limited wave growth during the Joint North Sea Wave Program (JONSWAP), relates the non-dimensional wave variance

$$
\tilde{\mathrm{e}}=\frac{\mathrm{eg}^{2}}{\mathrm{U}_{10}^{4}}
$$

with the wave age $\left(c_{p} / U_{10}\right)$. The parameter e is the variance of the surface wave field. Kitaigorodskii's (1962) non-dimensionalization was also used in some of the more recent investigations [Donelan, Hamilton \& Hui (1985), Dobson, Perrie \& Toulany 

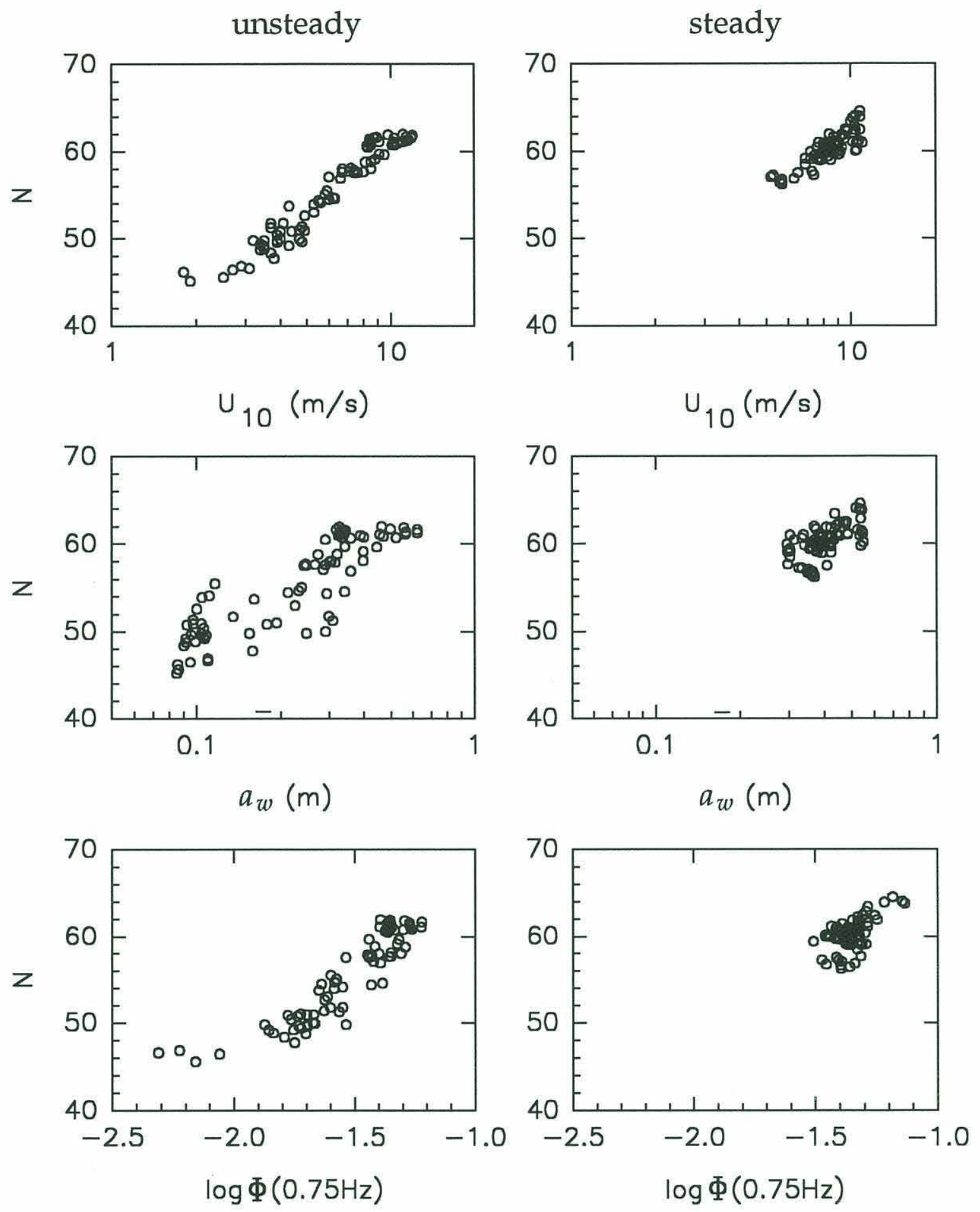

Figure 4.18. Plot of $\mathrm{N}(4.3 \mathrm{kHz})$ versus selected wind and wave parameters. The plots on the left are for data during unsteady conditions. The plots on the right are for data during steady conditions. 

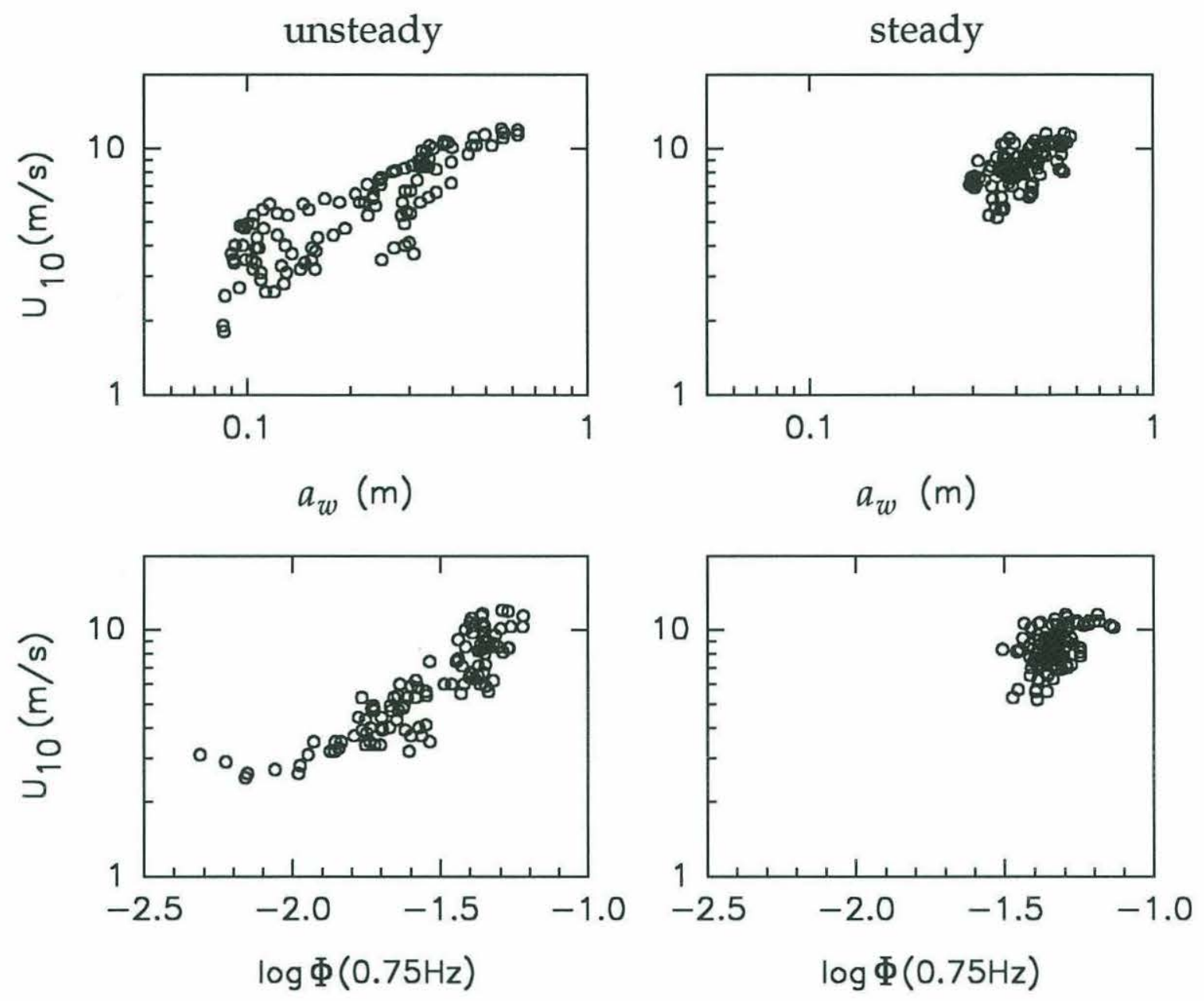

Figure 4.19. Plot of $U_{10}$ versus selected wave parameters. The plots on the left are for data during unsteady conditions. The plots on the right are for data during steady conditions. 
(1989), Donelan et al (1992)] on fetch limited wave growth in both lake and ocean environments.

Our wave measurements are complicated by the presence of swell whose characteristics are unrelated to the local wind conditions. Dobson et al (1989), whose measurements off the coast of Nova Scotia during the Canadian Atlantic Storms Program (CASP) were also contaminated by swell, suggested that the frequency separating swell and the wind sea portion of the spectrum $\mathrm{f}_{\mathrm{s}}$ is $0.03 \mathrm{~Hz}$ less the wind sea peak frequency. The total energy of the wind sea spectrum is therefore

$$
e=\int_{f_{s}}^{\infty} \Phi(f) d f
$$

Their approach is similar to our method of separating the wind wave spectrum from the swell components (Section 4.3). However, we defined $\mathrm{f}_{\mathrm{s}}$ to be the frequency of the minimum value of $\Phi(f)$ between the swell and wind sea peaks and its value was obtained by inspection.

Figure 4.20 shows a well correlated log-log plot of ẽ and the inverse wave age computed using the NOBS data. The correlation coefficient between the two parameters is 0.97 . Using linear orthogonal regression, we found that the relationship is best described by the expression

$$
\frac{\mathrm{eg}^{2}}{\mathrm{U}_{10}^{4}}=2.4 \times 10^{-3}\left(\frac{\mathrm{U}_{10}}{\mathrm{c}_{\mathrm{p}}}\right)^{-3.2}
$$


Table 4.6 compares our results with the experiments mentioned earlier in this section. Our results are close to those of the previous experiments, particularly to that of Donelan et al (1992). The JONSWAP result did not separate wind seas from swell. The agreement between our results and the results of the experiments given in Table 4.6 is particularly remarkable since the other experiments were made under fetch-limited conditions. Our results suggest that within experimental error, the empirical relationship between the wind and wave parameters expressed by Equation 4.16 is valid over a wide range of fetch lengths.

\subsubsection{Wave spectrum characteristics}

Using previous observational evidence, Phillips (1985) suggested that the portion of wave height spectrum $\Phi(\omega)$ above the spectral peak can be described by the equation

$$
\Phi(\omega)=\alpha u_{*} g \omega^{-4}
$$

Table 4.6. Coefficients of the non-dimensional wind and wave growth expression.

$$
\frac{\mathrm{eg}^{2}}{\mathrm{U}_{10}^{4}}=m\left(\frac{\mathrm{U}_{10}}{\mathrm{c}_{\mathrm{p}}}\right)^{n}
$$

\begin{tabular}{|l|c|c|c|}
\cline { 2 - 4 } \multicolumn{1}{c|}{} & $\boldsymbol{m}$ & $\mathbf{1 0}^{\mathbf{3}} \boldsymbol{n}$ & max. fetch $\mathbf{( k m )}$ \\
\hline This study & -3.2 & 2.4 & unlimited \\
\hline JONSWAP (1973) & -3.0 & 2.0 & $160+$ \\
\hline Donelan et al (1985) & -3.3 & 2.7 & 300 \\
\hline Dobson et al (1989) & -3.4 & 2.7 & 30 \\
\hline Donelan et al (1992) & -3.3 & 2.2 & 20 \\
\hline
\end{tabular}




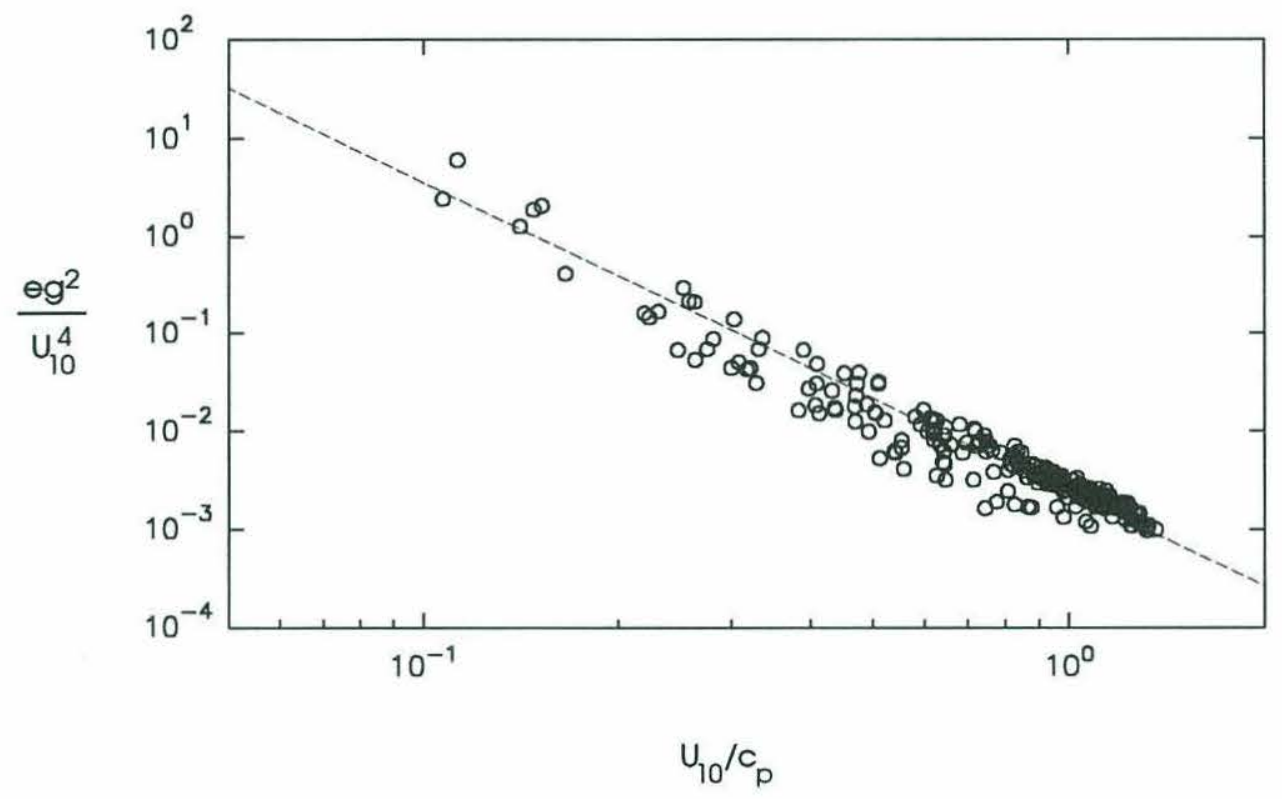

Figure 4.20. Plot of the non-dimensional wave energy versus the inverse wave age. 


\section{Chapter 4}
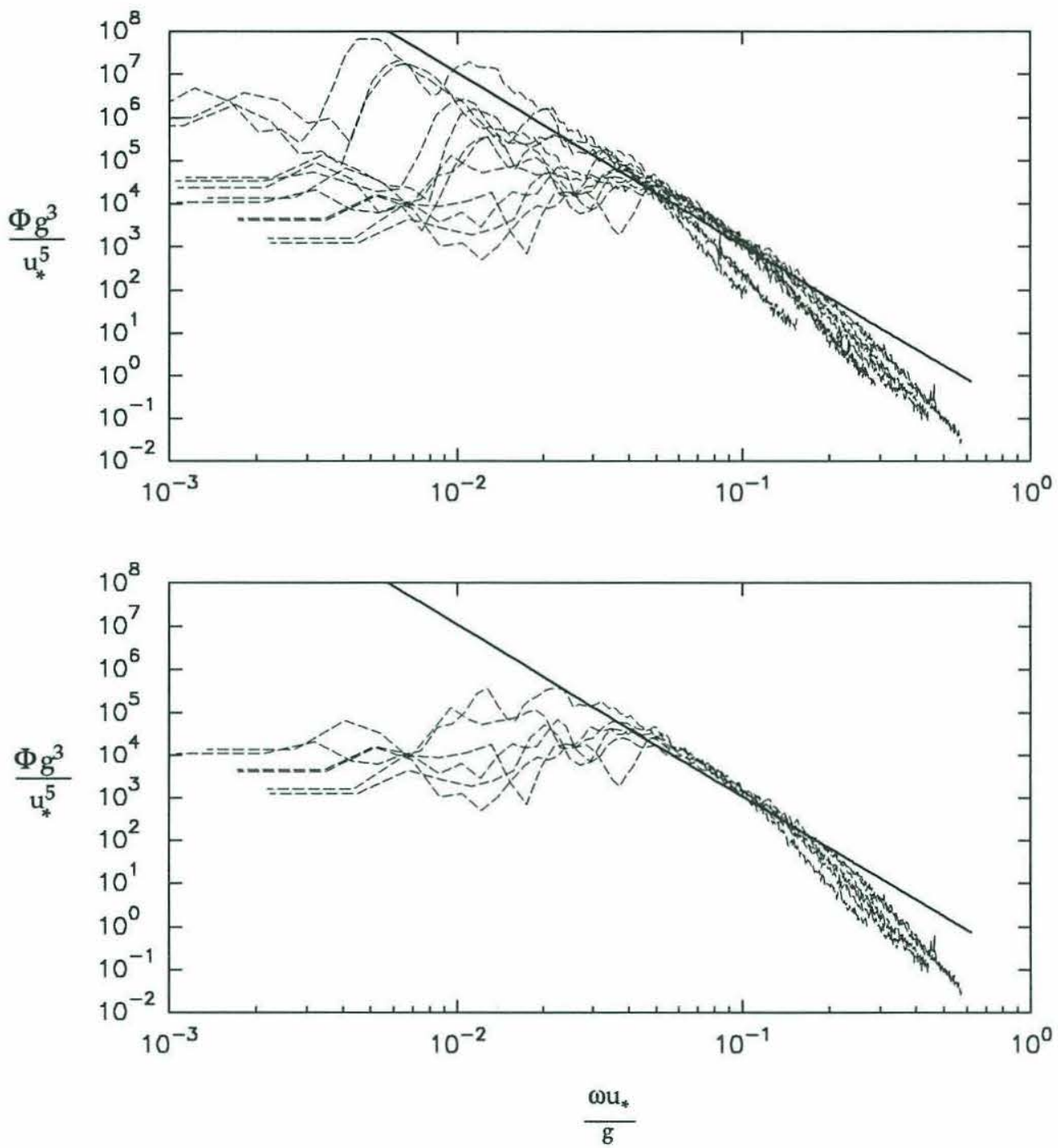

Figure 4.21. Selected normalized 1-hour wave spectra. The spectra were taken from wave height records sampled at 0000 UT. The solid line denotes $\Phi$ for $\alpha=0.11$ (Equation 4.17). a) Spectra from 12 day record. b) Spectra from fully developed seas. 


\section{Chapter 4}

where $\alpha$ is the Toba coefficient. Following Phillips, we nondimensionalized $\Phi(\omega)$ such that for an ideal spectrum of the form of Equation 4.17,

$$
\frac{\Phi g^{3}}{u_{*}^{5}}=\alpha\left(\frac{u_{*} \omega}{g}\right)^{-4}
$$

The term inside the parenthesis is the nondimensionalized radian frequency $\omega$. The plot of the $\log$ of equation 4.18 and $\log (\mathrm{u} * \omega / \mathrm{g})$ should have a straight line with slope $=-4$. Figure 4.21 a shows the 12 spectra in Figure 4.2 which were taken from one hour samples of the wave gauge record beginning at 0000 UT each day during the observation period. The solid line is for the idealized $\Phi(\omega)$ whose $\alpha=0.11$ (Equation 4.8). The plots show a close agreement between Equation 4.17 and the measured $\Phi(\omega)$ in the frequency range between $\omega_{\mathrm{p}}$ and approximately $2.5 \omega_{\mathrm{p}}$. For $\omega>\omega_{\mathrm{p}}$, the slope of the wave spectrum appears to be closer to $\Phi(\omega) \sim \omega^{-7}$. Figure $4.21 \mathrm{~b}$ shows typical wave spectra under fully developed conditions (Julian days 273-278). For $\omega>\omega_{p^{\prime}}$ the plots collapse into one spectrum.

Phillips (1985) also predicted that the upper limit of the $\omega^{-4}$ slope is determined by the presence of wind drift $\mathrm{c}_{\mathrm{q}} \approx \mathrm{u} *$ which would inhibit the creation of waves frequencies whose phase speed is comparable to that value (see Section 2.2). He proposed that the wavenumber of this upper limit is

$$
\mathrm{k}_{1}=\omega_{1}^{2} / \mathrm{g}=\mathrm{rg} / \mathrm{u}_{*}^{2}
$$

where $r$ is of order one. He subsequently estimated that $r \approx 0.16$ for typical wind wave conditions. Rearranging Equation 4.19 gives 


$$
\frac{\omega_{1} \mathrm{u}_{*}}{\mathrm{~g}}=\mathrm{r}^{0.5} \approx 0.4
$$

which is approximately 3 times the value suggested by Figure 4.21. This suggests that the formation of high frequency wind waves is inhibited by some mechanism whose phase speed is lower than $u_{*}$. It is currently not known what this mechanism is. It is also possible that Keulegan's (1951) measurements of the wind drift in a closed channel [which Phillips (1985) used] overestimates the value of $\mathrm{c}_{\mathrm{q}}$ in the open ocean. 


\section{SPECTRAL DISSIPATION ESTIMATES}

In Chapter 2, we described the basis of the Phillips (1985) and the KHH surface wave dissipation models. We showed that the two models differ in form, in their dependence on the wave spectrum, and in the method used to evaluate their coefficients. While Phillips (1985) is based on the assumption of a spatial and temporal equilibrium balance among the source terms in the energy transfer equation, $\mathrm{KHH}$ tuned the dissipation parameters with the wind input and nonlinear flux source functions to reproduce the behavior of fetch-limited wind wave growth. We also showed that we can derive simple, explicit dissipation equations by making general assumptions on the characteristics of the wave spectrum and substituting the wave spectrum equation into the full spectral dissipation expressions.

Table 5.1 summarizes the four spectral dissipation estimates discussed in Chapter 2. In this work, $d(\omega)$ denotes the spectral dissipation computed from the measured wave height spectrum while $\varepsilon(\omega)$ denotes the spectral dissipation computed from wind and integral wave parameters (reduced estimate). The total dissipation estimates are therefore

$$
\begin{aligned}
& \mathrm{D}=\int_{\omega} \mathrm{d}(\omega) d \omega \\
& \mathrm{E}=\int_{\omega} \varepsilon(\omega) d \omega
\end{aligned}
$$

In the subsequent sections of this chapter, we will examine the spectral behavior of these model and compare these estimates to ambient noise measurements. 
Table 5.1. Dissipation estimates (see Chapter 2 for full details.)

\section{1. spectral dissipation equations}

$$
\begin{aligned}
& \mathrm{d}_{\mathrm{P}}(\omega)=\frac{\mathrm{I}(3 p) \gamma}{16[\mathrm{I}(p)]^{3}} \rho_{\mathrm{w}} \mathrm{g}^{-3} \omega^{11} \Phi^{3}(\omega) \\
& \varepsilon_{\mathrm{P}}(\omega)=4 \gamma \beta^{3} \mathrm{I}(3 p) \rho_{\mathrm{w}} \mathrm{u}_{*}^{3} \omega^{-1} \\
& \mathrm{~d}_{\mathrm{H}}(\omega)=\rho_{\mathrm{w}} \mathrm{g} c_{H}(\omega / \bar{\omega})^{2}\left(\bar{\alpha} / \bar{\alpha}_{\mathrm{PM}}\right)^{2} \bar{\omega}_{w} \Phi(\omega) \\
& \varepsilon_{\mathrm{H}}(\omega)=0.89 \rho_{\mathrm{w}} \mathrm{g} c_{H}\left(\bar{\alpha} / \bar{\alpha}_{\mathrm{PM}}\right)^{2} a_{w}^{2} \bar{\omega}_{w}^{2} \omega^{-2}
\end{aligned}
$$

\section{2. total dissipation equations}

$$
\begin{aligned}
& \mathrm{D}_{\mathrm{P}}=\frac{\mathrm{I}(3 p) \rho_{\mathrm{w}} \mathrm{g}^{-3} \gamma}{16[\mathrm{I}(p)]^{3}} \int_{\omega_{p}}^{\infty} \omega^{11} \Phi^{3}(\omega) \mathrm{d} \omega \\
& \mathrm{E}_{\mathrm{P}}=2 \gamma \beta^{3} \mathrm{I}(3 p) \rho_{\mathrm{w}} \mathrm{u}_{*}^{3} \ln \left[\mathrm{r}\left(\mathrm{c}_{\mathrm{p}} / \mathrm{u}_{*}\right)^{2}\right] \\
& \mathrm{D}_{\mathrm{H}}=\rho_{\mathrm{w}} \mathrm{g} \int_{\omega_{o}}^{\infty} c_{H}\left(\omega / \bar{\omega}_{w}\right)^{2}\left(\bar{\alpha} / \bar{\alpha}_{\mathrm{PM}}\right)^{2} \bar{\omega}_{w} \Phi(\omega) \mathrm{d} \omega \\
& \mathrm{E}_{\mathrm{H}}=1.33 \rho_{\mathrm{w}} \mathrm{g} c_{H}\left(\bar{\alpha} / \bar{\alpha}_{\mathrm{PM}}\right)^{2} a_{w}^{2} \bar{\omega}_{w}
\end{aligned}
$$

\subsection{Experimental results.}

Although the four models $\left(\mathrm{D}_{\mathrm{P}}, \mathrm{E}_{\mathrm{P}}, \mathrm{D}_{\mathrm{H}}, \mathrm{E}_{\mathrm{H}}\right)$ are based on different assumptions regarding the spectral description of dissipation, the form of the wave height spectrum, and the existence of an equilibrium wave spectrum, these equations give comparable estimates of the total dissipation for most of the measured wind and wave condition. This is observed in Figure 5.1 which compares the time series of the four dissipation estimates during the NOBS observation period. 


\section{Chapter 5}

Figure 5.1a shows very good agreement between the full and reduced $\mathrm{KHH}$ dissipation estimates $\mathrm{D}_{\mathrm{H}}$ and $\mathrm{E}_{\mathrm{H}}$. The two time series are virtually indistinguishable through most of the observation period. Figure $5.1 \mathrm{~b}$ shows a comparison between the time series of $D_{P}$ and $E_{P}$. Here we see that while there is good agreement between these two estimates over most of the data, there can be significant differences between them especially during periods of wind and wave growth and decay. These differences are explained further in Section 5.1.2. Figure 5.1c compares the KHH and Phillips dissipation estimated from the measured spectra $D_{P}$ and $D_{H}$. In this figure, we see that $D_{P}$ is consistently higher by a small factor than $D_{H}$. This difference is nearly constant. This is because the magnitude of the coefficient of $D_{P}$ is slightly larger than that of $\mathrm{D}_{\mathrm{H}}$. Note however that this difference is small compared to the dynamic range of the data.

These time series comparisons (Figures 5.1a-d) show that the different estimates agree well under steady wind and wave conditions but may vary significantly during periods of growth or decay. This result is not surprising since the coefficients of the different dissipation expressions were chosen such that the dissipation, the wind input and the nonlinear flux expressions are of comparable order. Note that even though the influence of wind speed on the Snyder et al (1981) wind input formula [used by KHH] (Equation 1.14) is slightly different from the Plant (1982) wind input formula [used by Phillips (1985)] (Equation 1.15), these two formulas give values that are comparable to each other in the range $\mathrm{f}<1 \mathrm{~Hz}$ (Figure 1.9). Hence, the Phillips (1985) and KHH dissipation estimates are consequently of comparable order. 


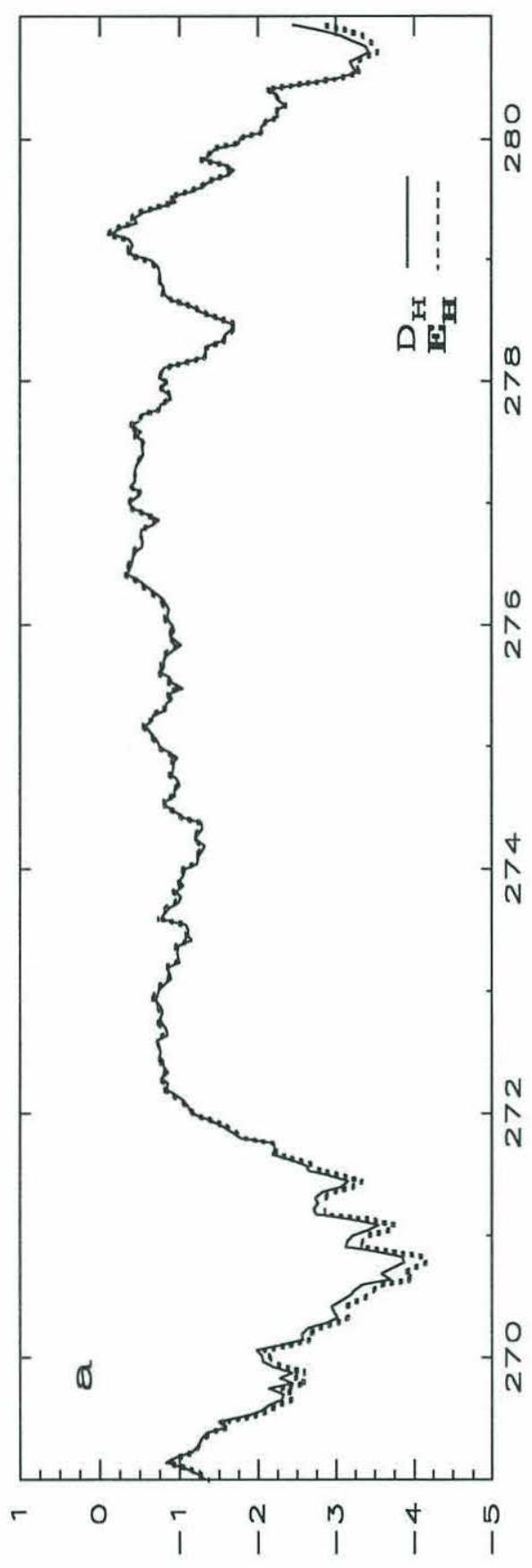

(uo!̣do!sSL!p) 6o|

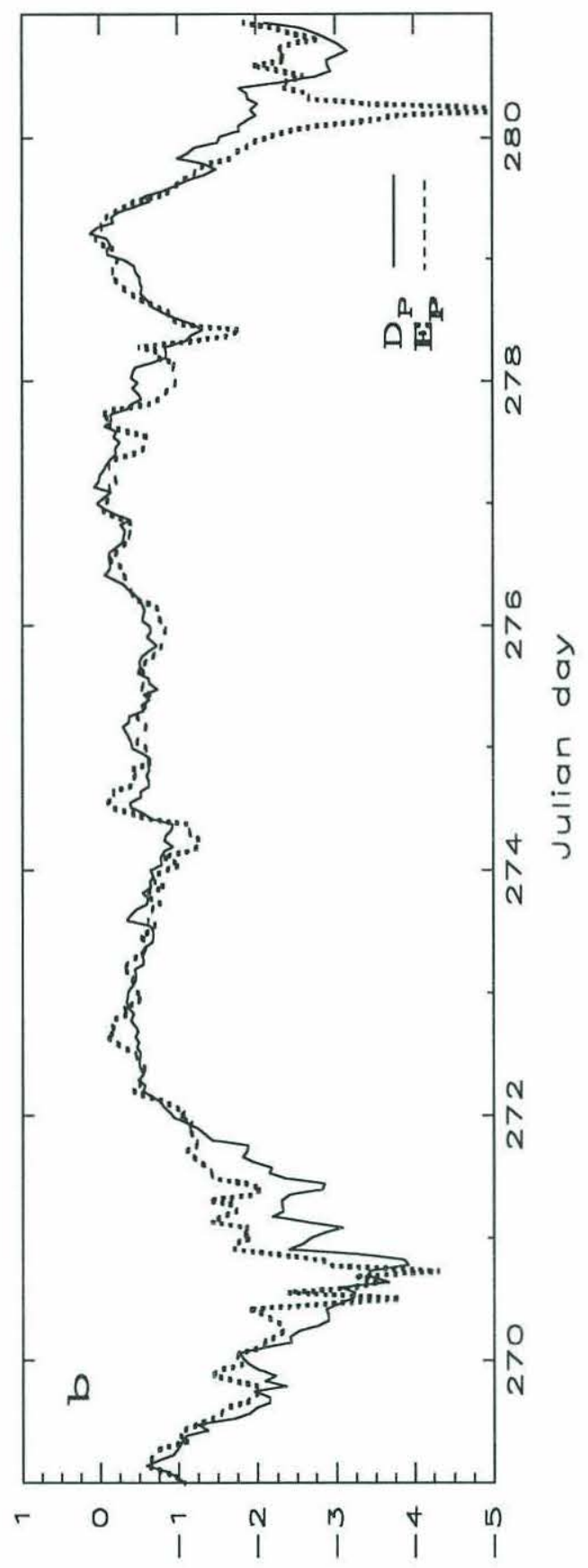

(uonpodiss!ip) 60|

Figure 5.1. Comparison of the time series of the $\log$ of the total dissipation $\left[\mathrm{kg} / \mathrm{s}^{3}\right]$ computed using the formulas given in Table 5.1. a) $\mathrm{D}_{\mathrm{H}}$ (solid line) and $\mathrm{E}_{\mathrm{H}}$ (short dash). b) $D_{\mathrm{P}}$ (solid line) and $\mathrm{E}_{\mathrm{P}}$ ( short dash). 


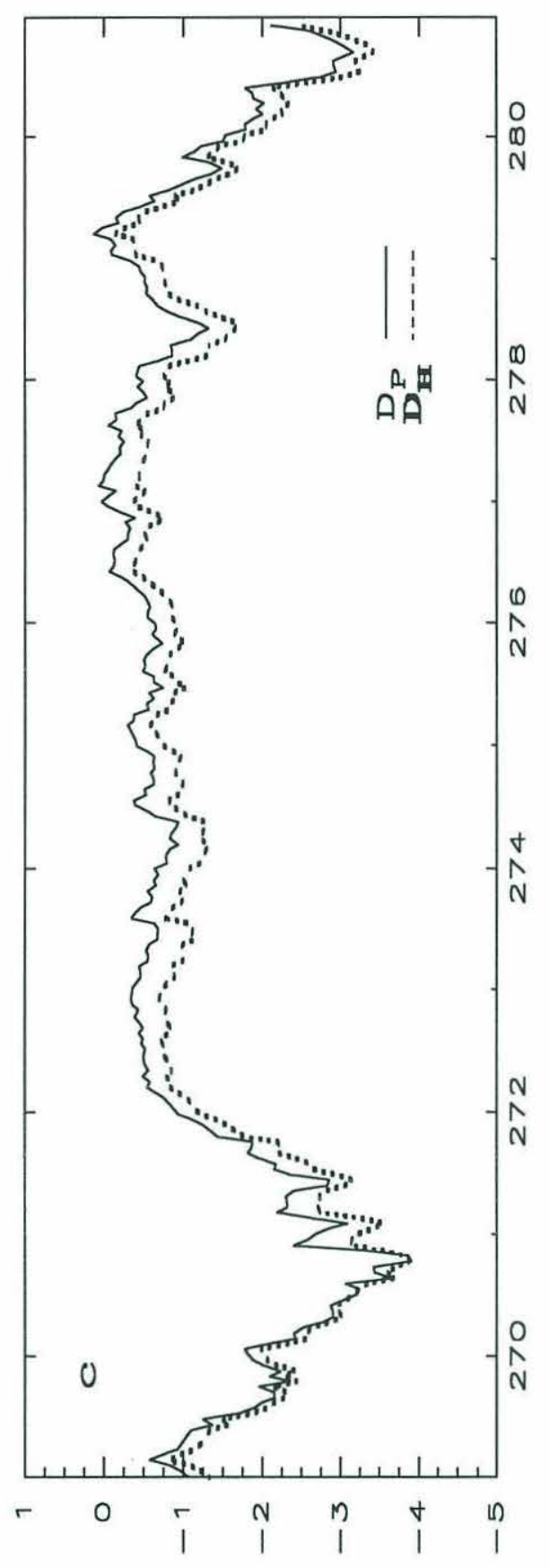

(uol!pdissip) 60|

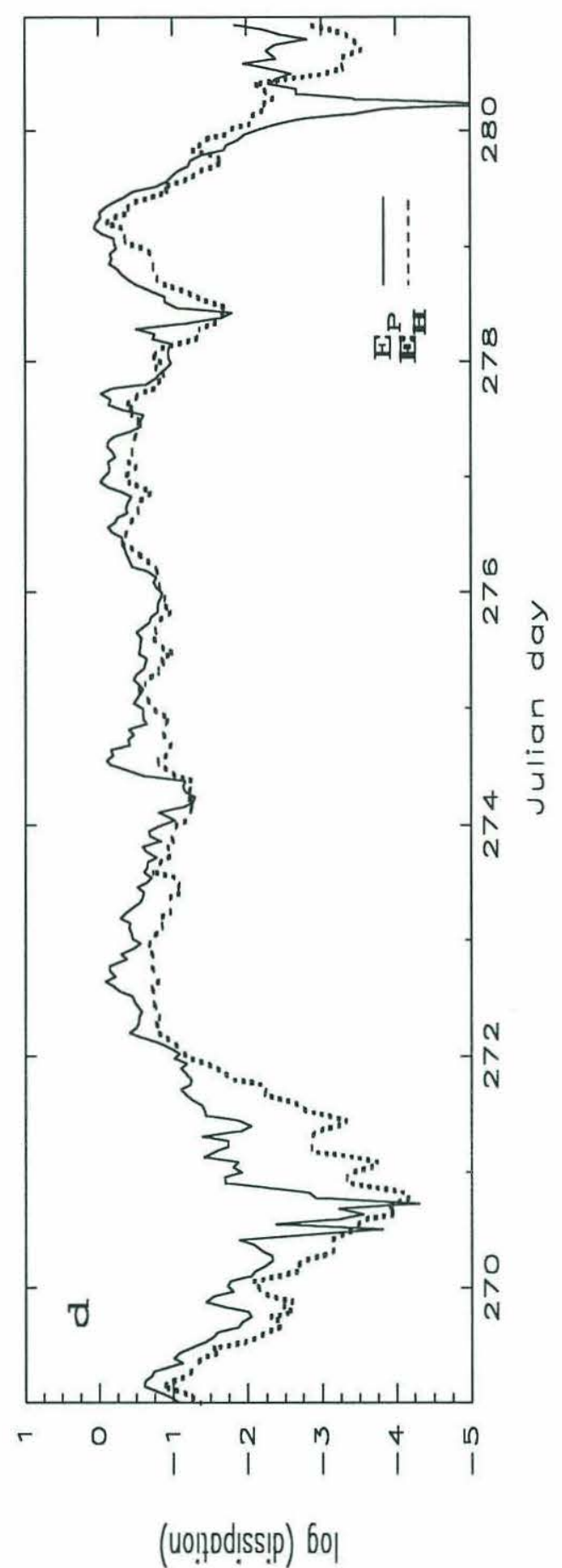

(uon!pdiss!̣) bo|

Figure 5.1. Continued from last page. c) $D_{P}$ (solid line) and $D_{H}$ (short dash). d) $E_{P}$ (solid line) and $\mathrm{E}_{\mathrm{H}}$ ( short dash). 


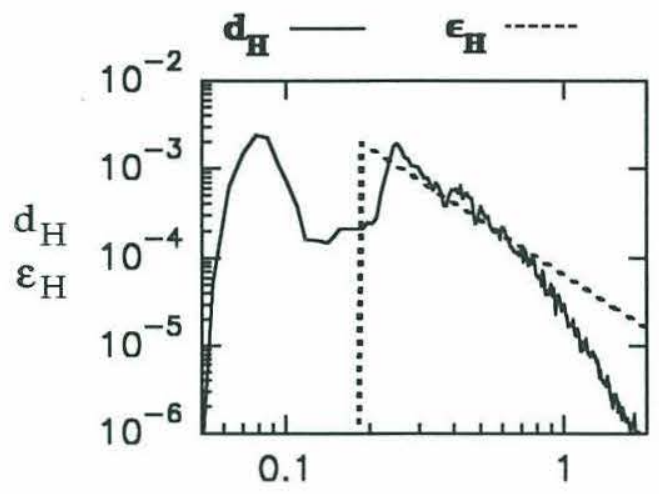

a) 269

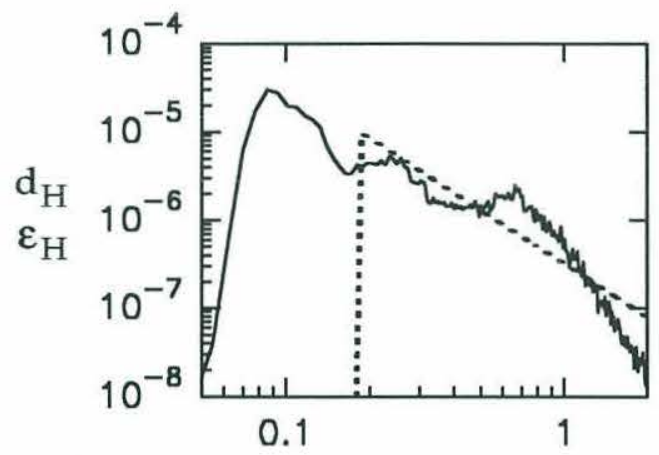

c) 271

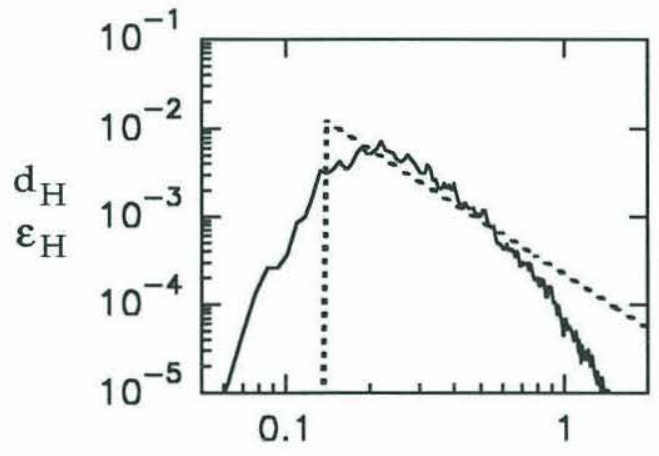

e) $273 \quad \mathrm{f}(\mathrm{Hz})$

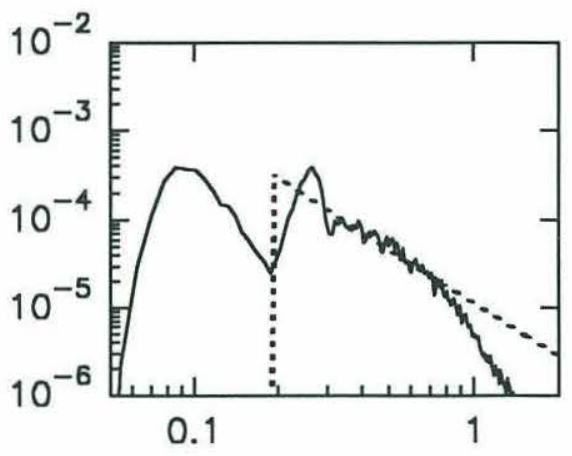

b) 270

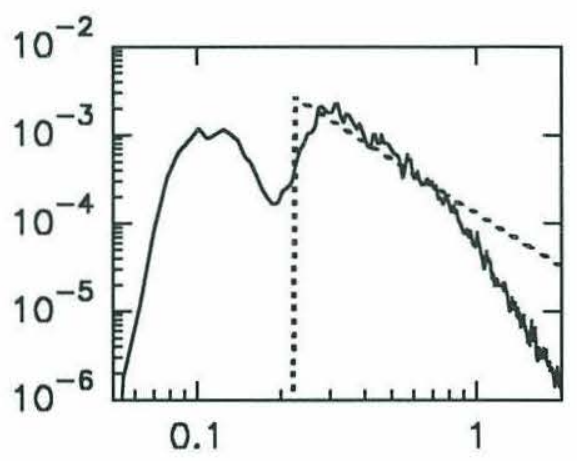

d) 272

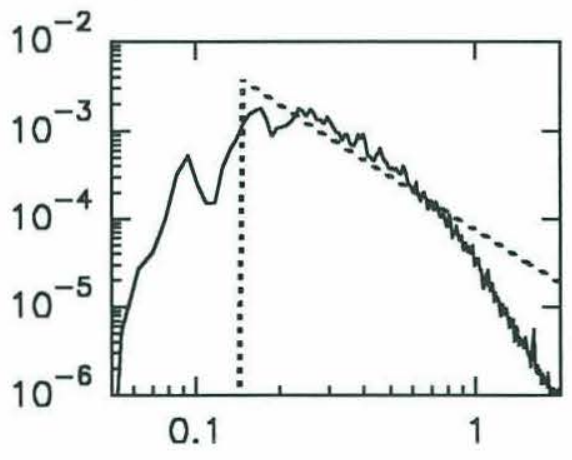

f) $274 \quad \mathrm{f}(\mathrm{Hz})$

Figure 5.2. Plots showing the spectral dependence of the $\mathrm{KHH}$ dissipation $\mathrm{d}_{\mathrm{H}}(\mathrm{f})$ and $\varepsilon_{\mathrm{H}}(\mathrm{f})\left[\mathrm{kg} / \mathrm{s}^{3} \mathrm{~Hz}\right]$. These spectral estimates were computed from the wave spectra shown in Figure 4.2. Numbers below the plots indicate the Julian day from which the plots were taken. 

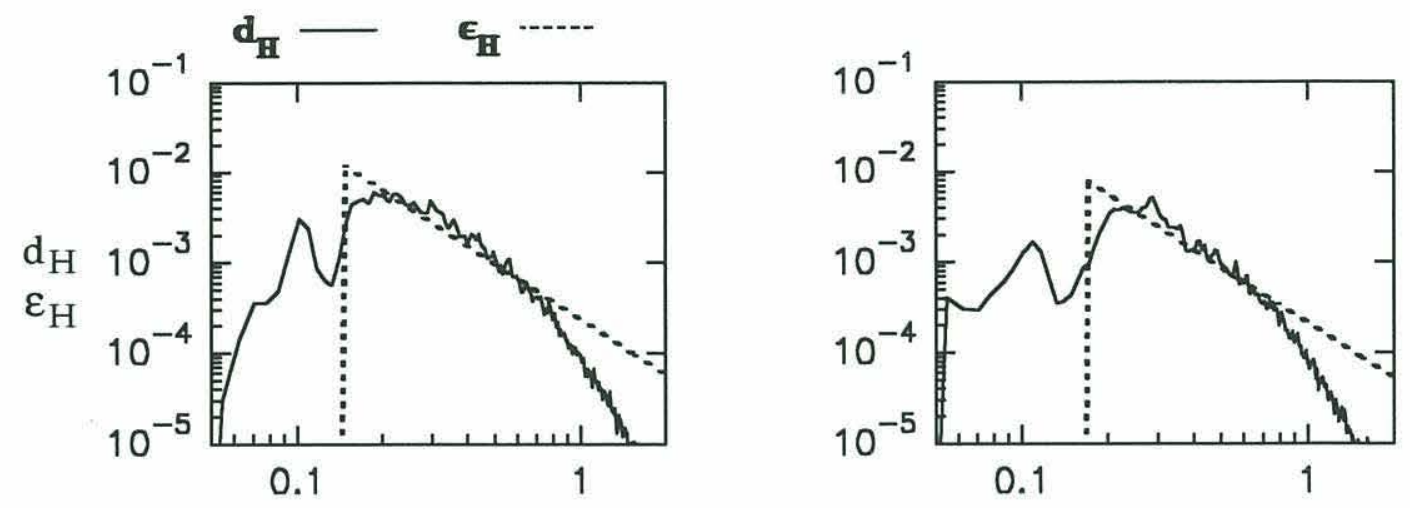

g) 275

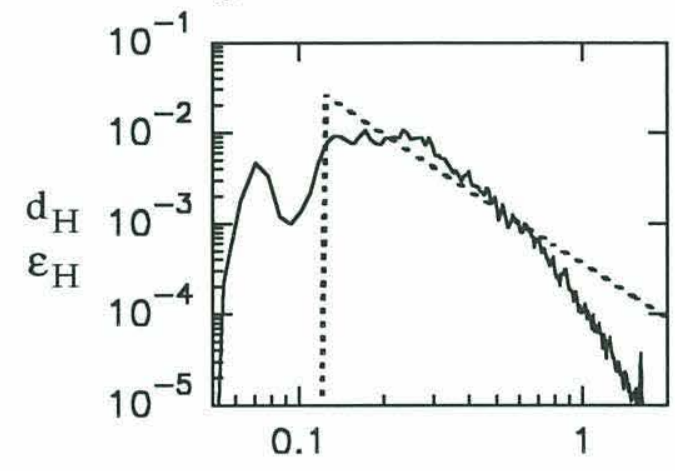

h) 276

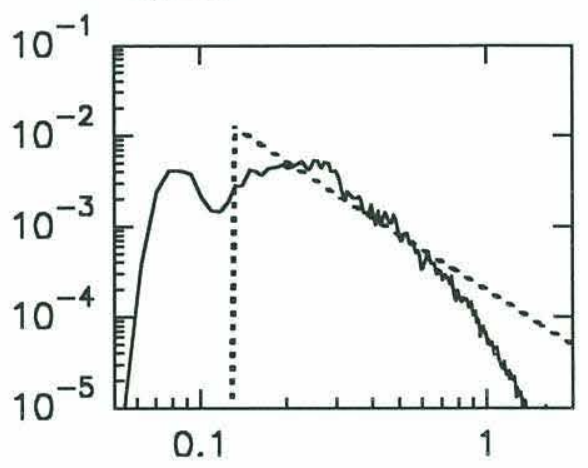

i) 277

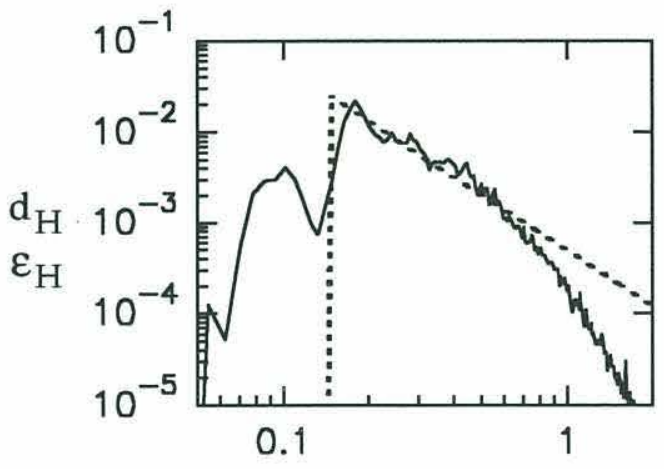

j) 278

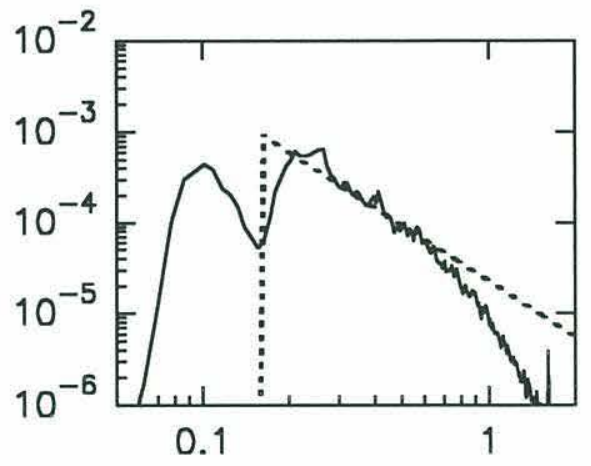

k) $279 \quad \mathrm{f}(\mathrm{Hz})$

1) $280 \quad \mathrm{f}(\mathrm{Hz})$

Figure 5.2. Continued from last page.

\subsection{1 $D_{H}$ and $E_{H}$.}




\section{Chapter 5}

In the discussion in Section 2.4, we showed how $\mathrm{E}_{\mathrm{H}}$ was derived by substituting an idealized $\omega^{-4}$ spectrum into $d_{H}$. The mean frequency $\bar{f}$ and the variance $a^{2}$ of the idealized spectrum are equal to those of the wind wave portion of the measured wave spectrum. In Figure 5.2, we show the spectral dependence of the dissipation $d_{H}(f)$ and $\varepsilon_{\mathrm{H}}(\mathrm{f})$ for the twelve wave height spectra shown in Figure 4.2. The solid line plot indicates dissipation computed using the measured wave spectrum while the dashed line indicates the spectral dissipation using the idealized wave height spectrum

$$
\Phi_{\mathrm{M}}(\omega)=3 a_{w}^{2} \omega_{\mathrm{p}}^{3} \omega^{-4}
$$

The spectral estimates are very similar both in magnitude and spectral slope in much of the wind wave region $(\mathrm{f}<1 \mathrm{~Hz})$. Above $1 \mathrm{~Hz}$, the slopes of the measured wave and dissipation spectra are steeper. While the influence of the discrepancy in the higher frequencies on the total dissipation is small, it does lead to an underestimate of the peak frequency $\omega_{\mathrm{p}}$ since we assumed that the spectral slope is constant throughout when in fact, the measured wave spectrum has an $\omega^{-4}$ slope from the spectral peak to approximately $1 \mathrm{~Hz}$ and a steeper $\omega^{-7}$ slope above $1 \mathrm{~Hz}$..

Much of the difference between $\mathrm{D}_{\mathrm{H}}$ and $\mathrm{E}_{\mathrm{H}}$ arises because we chose not to model the dissipation below the $\varepsilon_{\mathrm{H}}$ wind wave spectral peak. We see from Figure 5.2 that $\mathrm{d}_{\mathrm{H}}(\mathrm{f})$ gives values of dissipation due to swell that are significant. Although the agreement in the wind wave spectral range is very good, the reduced formula $\mathrm{E}_{\mathrm{H}}$ completely neglects the dissipation in the swell region computed in $\mathrm{D}_{\mathrm{H}}$. While the total energy in the swell region is a significant proportion of $\Phi(\omega)$ and in some cases contains more energy than wind waves (see sample spectra in Figure 4.2), its contribution to the total 


\section{Chapter 5}

dissipation is comparably less because the contribution of the wave frequency components is weighted by $\omega^{2}$ (see Equations 2.18 or 2.19) which favors the higher frequencies.

It is also important to point out that $\mathrm{KHH}$ implicitly assumed a single peak spectrum in deriving the wave evolution equations. Their approach did not factor in the influence of swell on the dissipation equation. Since their model assumes that dissipation in deep water is due to wave breaking and since swell does not break (although swell may induce breaking of wind waves), extending the KHH-based formulas to include dissipation in the swell frequency range may overestimate the total dissipation. Nonetheless, the two time series in Figure 5.1a show that this difference is small compared to the dynamic range of the total dissipation.

\subsection{2 $D_{P}$ and $E_{P}$}

Clearly, the difference in the $\mathrm{D}_{\mathrm{P}}$ and $\mathrm{E}_{\mathrm{P}}$ estimates is more substantial than that between $D_{H}$ and $E_{H}$. This is because $E_{P}$ uses an equilibrium spectrum (Equation 2.8) assumption to model the measured wave spectrum while the $\mathrm{E}_{\mathrm{H}}$ model spectrum $\Phi_{\mathrm{M}}(\omega)$ is computed from the integral characteristics of the measured spectrum itself. The coefficients of $\Phi_{M}$ were chosen such that the measured and the idealized spectra have the same energy. Hence, the validity of our wave spectrum assumptions in computing $\mathrm{E}_{\mathrm{H}}$ from the integral wind wave spectrum is based only on whether or not the slope of the wind wave portion of the measured spectrum $\Phi$ is close to $\omega^{-4}$. The variance $a^{2}$ and mean frequency $\overline{\mathrm{f}}$ of $\Phi_{\mathrm{M}}$ are identical to those of $\Phi$. On the other hand, using $\Phi_{\mathrm{E}}$ (Equation 2.8) to compute $\mathrm{E}_{\mathrm{P}}$ is based primarily on the validity of two assumptions: that the spectral slope of the equilibrium spectrum is indeed $\omega^{-4}$, 


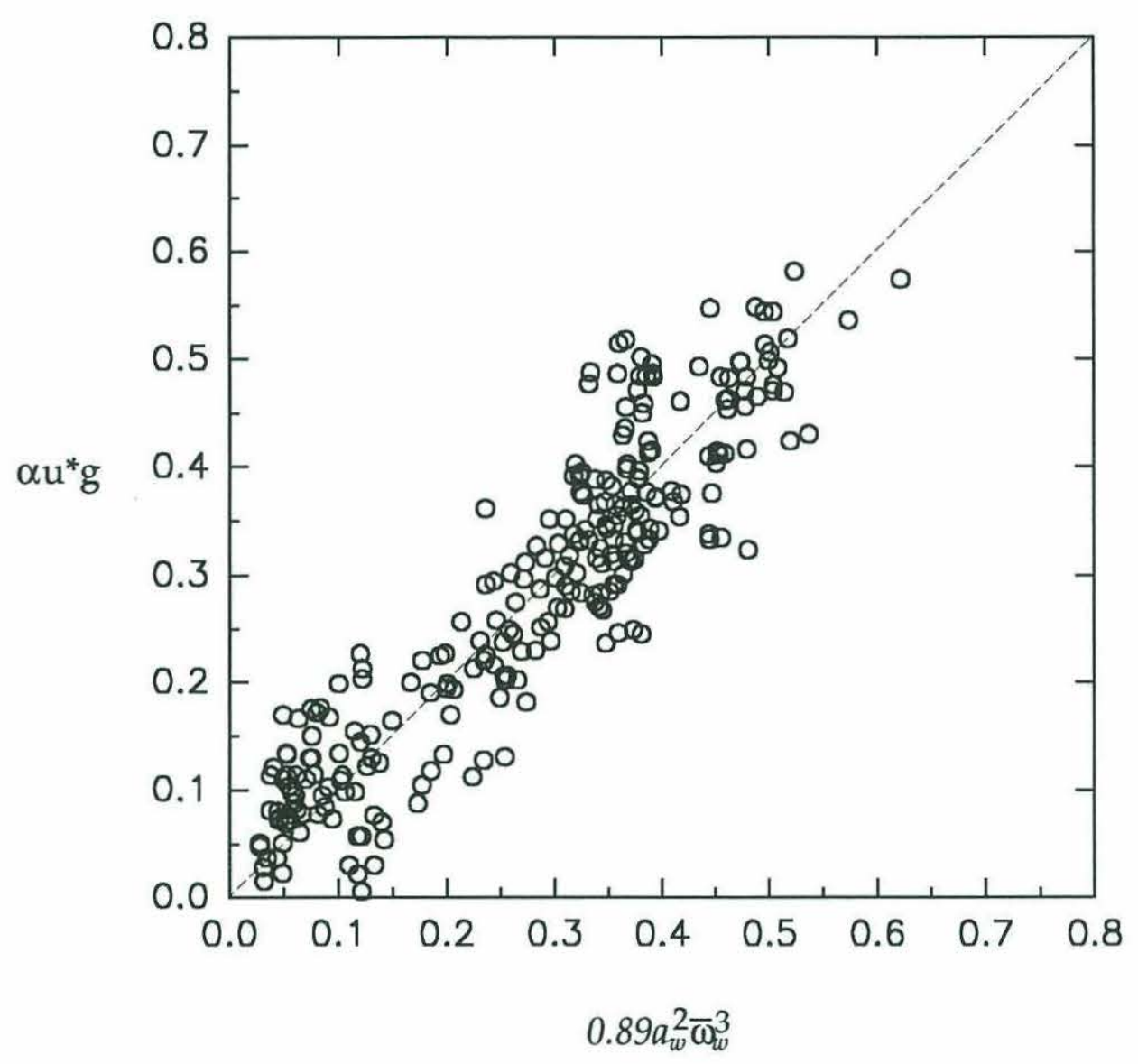

Figure 5.3. Plot showing a good correlation between $0.89 a_{w}^{2} \bar{\omega}_{w}^{3}$ and $\alpha \mathrm{u} * \mathrm{~g}$. The regression line has a slope $=1$ and the correlation coefficient between the two parameters is 0.92 . 


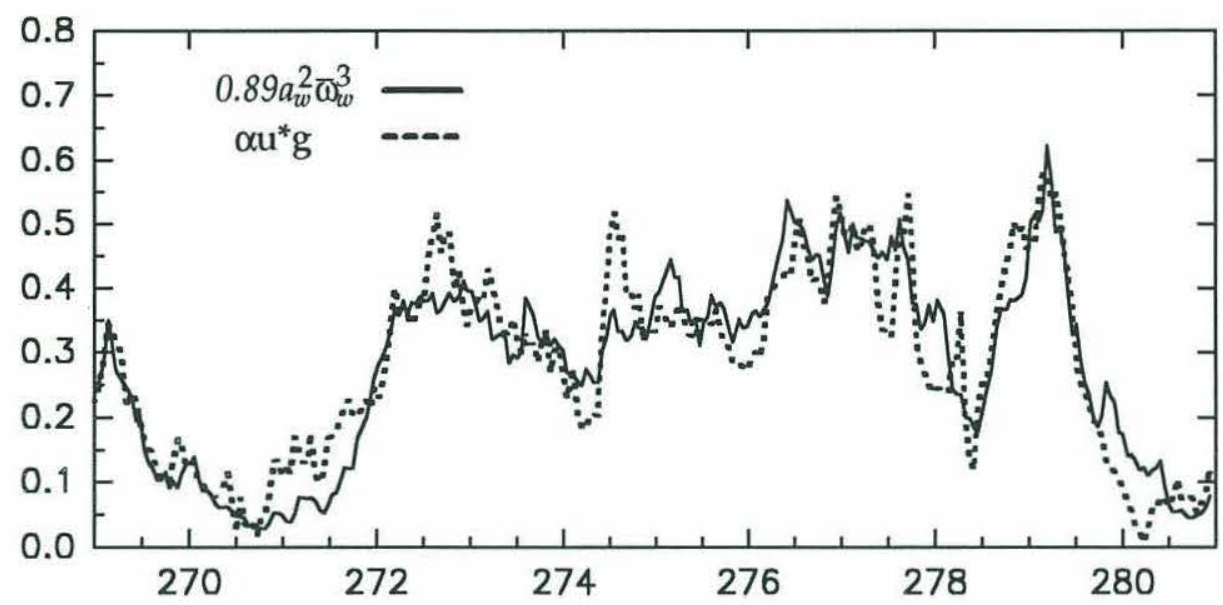

a)

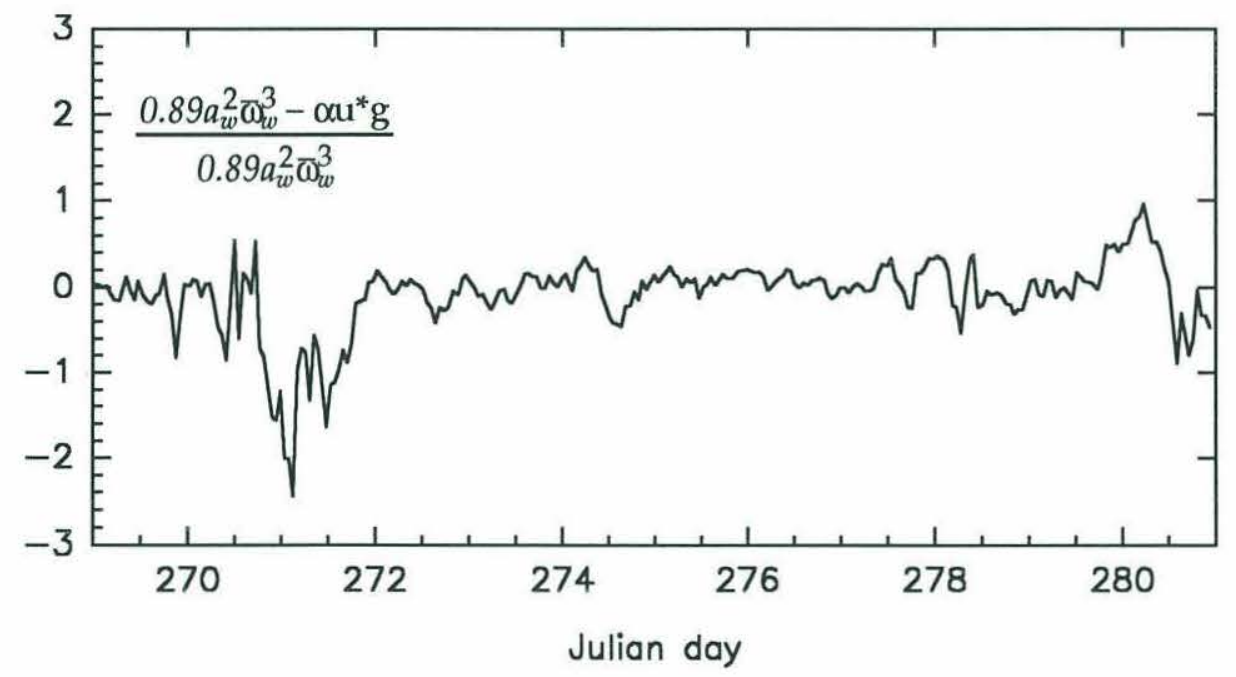

b)

Figure 5.4. a) Plot showing the time series of the $\Phi_{\mathrm{M}}$ and $\Phi_{\mathrm{E}}$ coefficients. $0.89 a_{w}^{2} \bar{\omega}_{w}^{3}$ (solid line) and $\alpha \mathrm{u}_{*} \mathrm{~g}$ (short dash) $\left[\mathrm{m}^{2} / \mathrm{s}^{3}\right]$. b) Plot showing the relative magnitude of the difference between $0.89 a_{w}^{2} \bar{\omega}_{w}^{3}$ and $\alpha \mathrm{u}_{*} \mathrm{~g}$. 
and that the coefficient of $\omega^{-4}$ in $\Phi$ is $\alpha u_{*} \mathrm{~g}$. The second assumption is also equivalent to the assumption that the wind input, dissipation and nonlinear spectral flux are in equilibrium balance under all wind and wave conditions.

In Figure 4.21, we showed a normalized plot comparing the twelve measured spectra in Figure 4.2 with Phillips' (1985) equilibrium spectrum $\Phi_{\mathrm{E}}$ (Equation 2.8). In that figure, we saw that the magnitude and slope of the measured wave spectrum $\Phi(\omega)$ are comparable to those of $\Phi_{\mathrm{E}}(\omega)$ between the wind wave peak and approximately $1 \mathrm{~Hz}$. We can explore the relationship further here. The equilibrium spectrum is

$$
\Phi_{\mathrm{E}}=\alpha \mathrm{u}_{*} \mathrm{~g} \omega^{-4}
$$

where $\alpha=0.11$ (see also Section 2.2). In Section 2.4, we showed that the idealized spectrum

$$
\Phi_{\mathrm{M}}(\omega)=3 a_{w}^{2} \omega_{\mathrm{p}}^{3} \omega^{-4}
$$

gives a spectrum whose integral characteristics are equal to those of the measured wave spectrum. We modify Equation 2.24 by substituting Equation 2.5 to express it in terms of $\bar{\omega}_{w}$ and get

$$
\Phi_{M}(\omega)=0.89 a_{w}^{2} \bar{\omega}_{w}^{3} \omega^{-4}
$$

Note that Figure 5.2 shows that this equation does well in modeling the measured wind wave spectrum. 
The variables in the coefficient of Equation 5.1 are the sea surface variance $a_{w}^{2}$ and the mean wave frequency $\bar{\omega}_{w}$ while in the coefficient of $\omega^{-4}$ in Equation $2.8, \mathrm{u} *$ is a variable while both $\alpha$ and g are constants. We can examine how effective $\Phi_{\mathrm{E}}$ is in modeling the measured wave spectrum by comparing the coefficients of $\Phi_{\mathrm{E}}$ and $\Phi_{\mathrm{M}}$. Figure 5.3 shows a plot of $0.89 a_{w}^{2} \bar{\omega}_{w}^{3}$ versus $\alpha \mathrm{u}_{*} \mathrm{~g}$ using the data from the NOBS observation period. The figure shows that the two parameters are well correlated (correlation coefficient $=0.92$ ). The dashed line indicates a plot of slope $=1$. The equation of the orthogonal linear regression of the data is

$$
\begin{gathered}
{\left[\alpha u_{*} g\right]=\left[0.89 a_{w}^{2} \bar{\omega}_{w}^{3}\right]+0.0017,} \\
{\left[\alpha u_{*} g\right] \approx\left[0.89 a_{w}^{2} \bar{\omega}_{w}^{3}\right] .}
\end{gathered}
$$

This shows that $\Phi_{\mathrm{E}}$ is, on average, a reasonable approximation to $\Phi$. It is important to point out however that the scatter of the data around Equation 5.2 (Figure 5.3) suggests that we incorporate a small error when we use this approximation. The nature of the difference is seen in Figure 5.4 which shows a plot of the time series of $0.89 a_{w}^{2} \bar{\omega}_{w}^{3}$ and $\left[\alpha \mathrm{u}_{*} \mathrm{~g}\right]$. Although the plot shows good agreement between the two parameters, there clearly are differences between the two. This is demonstrated in Figure $5.4 \mathrm{~b}$ which plots the difference between $0.89 a_{w}^{2} \bar{\omega}_{w}^{3}$ and $\left[\alpha \mathrm{u}_{*} \mathrm{~g}\right]$ normalized by $0.89 a_{w}^{2} \bar{\omega}_{w}^{3}$. From this plot, we see that $\left[\alpha \mathrm{u}_{*} \mathrm{~g}\right]$ significantly overestimates $0.89 a_{w}^{2} \bar{\omega}_{w}^{3}$ during the early stages of wind and wave growth (Julian day 271). When the wind is decaying (Julian day 279-280), [ $\left.\alpha \mathrm{u}_{*} \mathrm{~g}\right]$ significantly underestimates $0.89 a_{w}^{2} \bar{\omega}_{w}^{3}$. The time series also show that even under fully developed conditions, the wave field $0.89 a_{w}^{2} \bar{\omega}_{w}^{3}$ may not respond quickly to rapid fluctuations in the wind speed $\left[\alpha u_{*} g\right]$. 


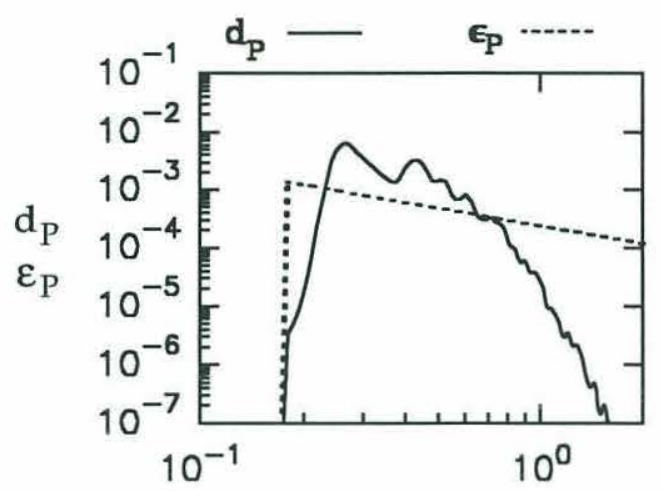

a) 269

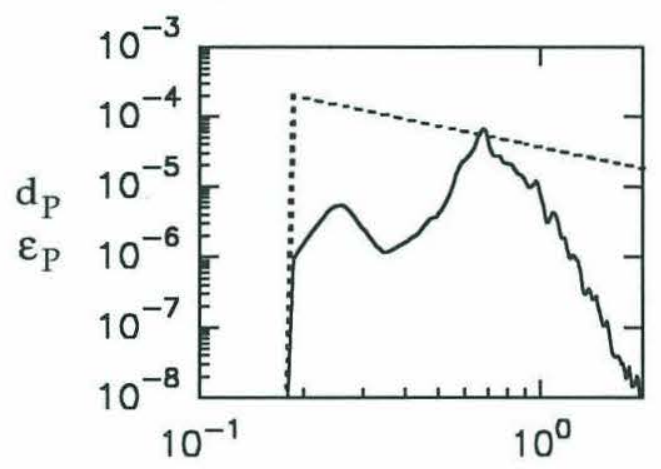

c) 271

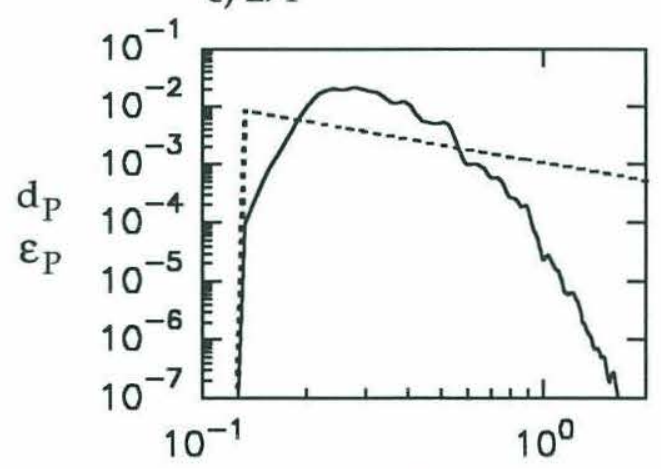

e) $273 \quad \mathrm{f}(\mathrm{Hz})$

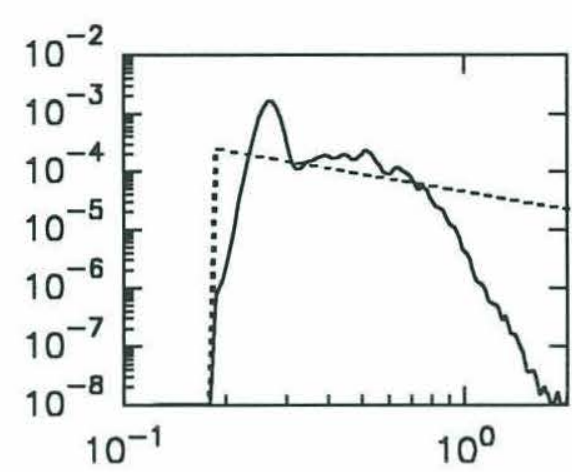

b) 270

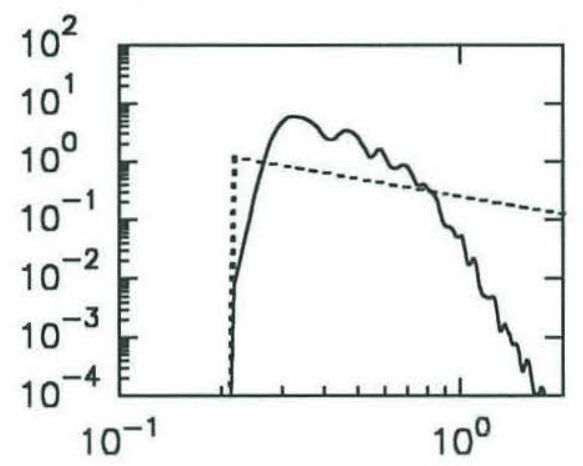

d) 272

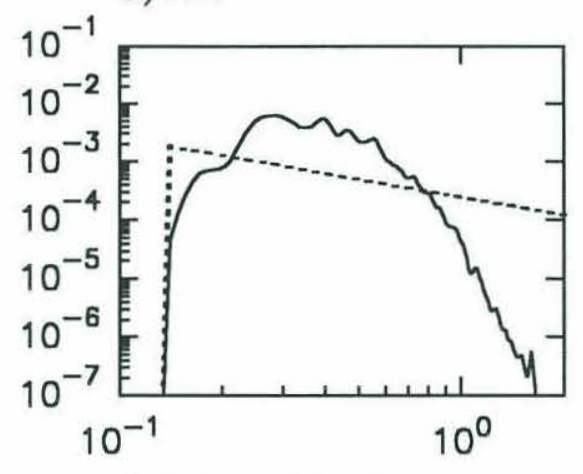

f) $274 \quad \mathrm{f}(\mathrm{Hz})$

Figure 5.5. Plots showing the spectral dependence of the Phillips dissipation $d_{P}(f)$ and $\varepsilon_{\mathrm{P}}(\mathrm{f})\left[\mathrm{kg} / \mathrm{s}^{3} \mathrm{~Hz}\right]$. The spectral estimates were computed from the wave spectra shown in Figure 4.2. Numbers below the plots indicate the Julian day from which the plots were taken. 


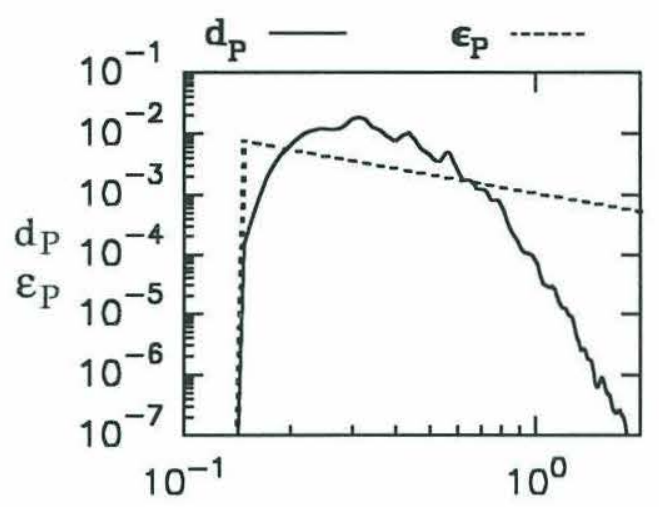

g) 275
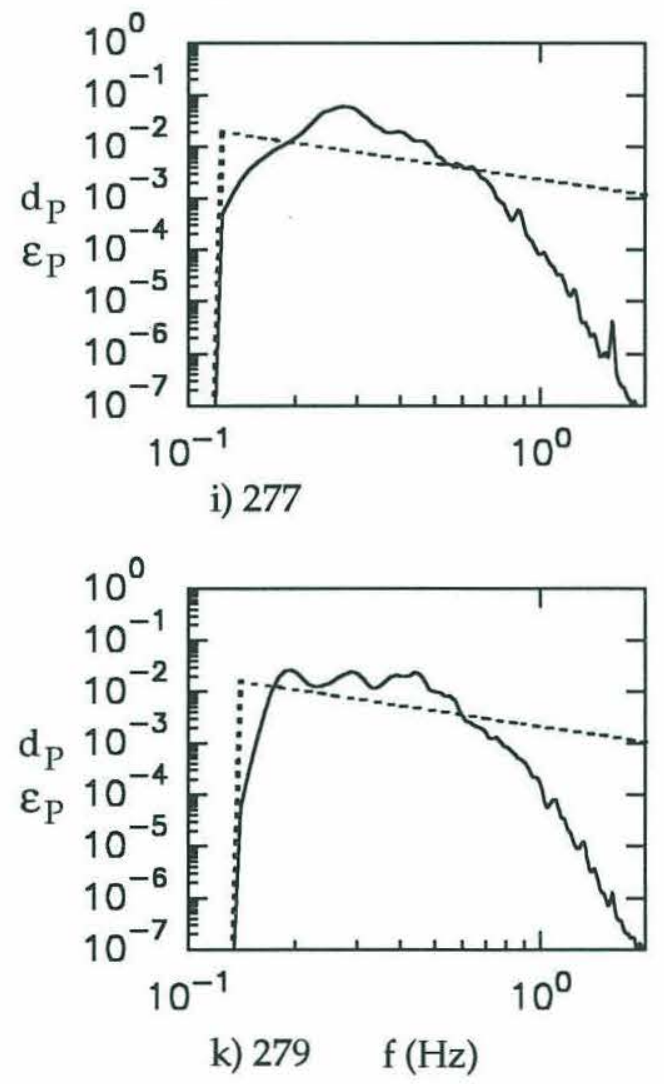

Figure 5.5. Continued from last page.

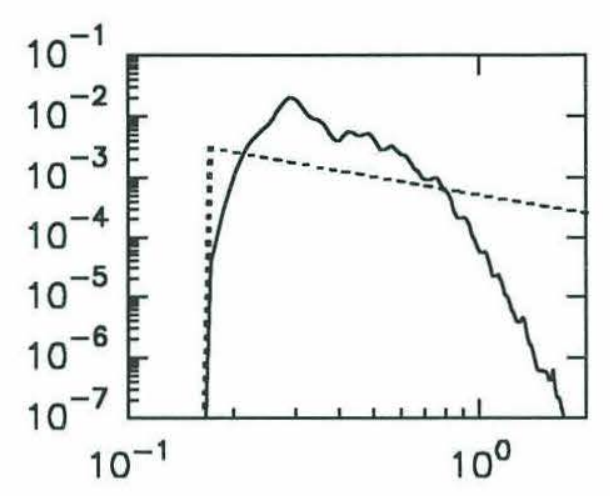

h) 276
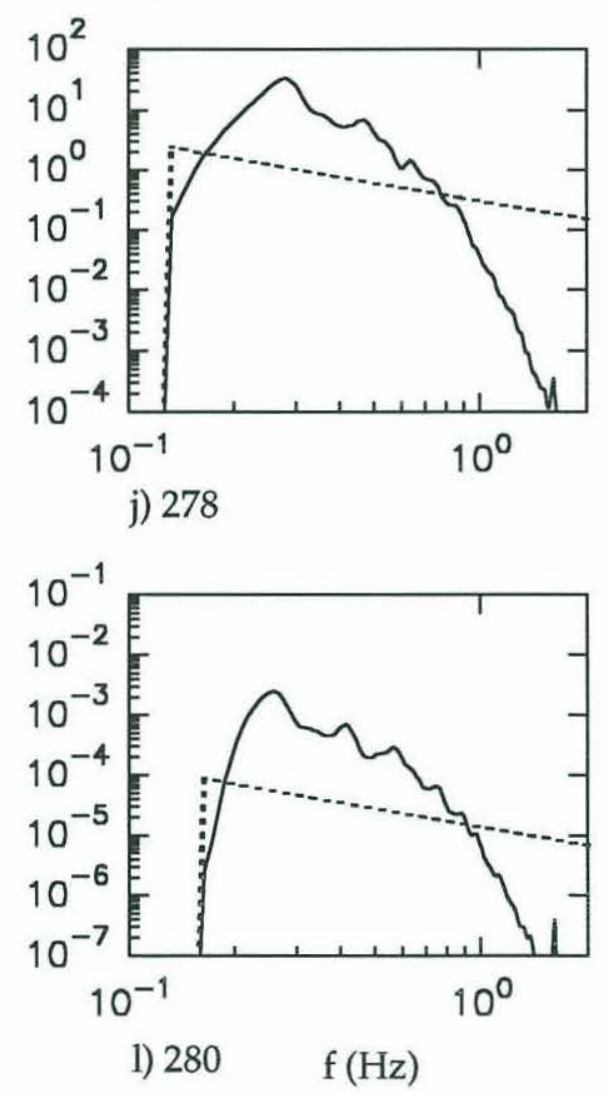
The plots in Figure 5.4 suggest that under fully developed or slowly varying wind and wave conditions, Phillips' (1985) equilibrium spectrum (Equation 2.8) adequately describes the wave field. Under conditions of rapid wind change, the assumption of local spatial and temporal equilibrium may not hold and the balance among the source terms (Equation 2.6) may not be strictly valid.

In Figure 5.5, logarithmic scale plots of the spectral dependence of the Phillips (1985) dissipation are shown. Similar to Figure 5.2, the plots compare the dissipation estimates computed from the measured spectra $\Phi$ and the model spectra $\Phi_{\mathrm{E}}$. Here we note that the difference between the two spectral estimates is larger in Figure 5.5 than in Figure 5.2. This could appear surprising given that Figures 4.21 and 5.3 show that $\Phi$ and $\Phi_{\mathrm{E}}$ agree well.

The difference is mainly due to the cubic dependence of $d_{P}$ and $\varepsilon_{P}$ on $\Phi$ and $\Phi_{E}$ respectively. This makes them more sensitive to differences between the values of the of the two spectra. For example, if $\Phi\left(\omega_{0}\right)=1.0$ and $\Phi_{\mathrm{E}}\left(\omega_{0}\right)=1.1$, then $\Delta \Phi\left(\omega_{0}\right)=0.1$ or a difference of $10 \%$. However, $\Phi^{3}\left(\omega_{0}\right)=1.0$ and $\Phi_{\mathrm{E}}{ }^{3}\left(\omega_{0}\right)=1.33$ gives a much larger difference of $33 \%$. Hence, although $\Phi(\omega)$ and $\Phi_{E}(\omega)$ are close to each other over a significant portion of the wind wave frequency range, the cubic wave spectrum dependence magnifies the difference between $d_{\mathrm{P}}$ and $\varepsilon_{\mathrm{P}}$.

The spectral behavior of the Phillips (1985) dissipation also explains why $\mathrm{E}_{\mathrm{P}}$ is initially significantly larger than $\mathrm{D}_{\mathrm{P}}$ during wind wave growth. The directional wave spectra plots (Figure 4.7 and 4.8) suggest that during the initial rapid increase in wind speed, the higher frequency waves energy achieve equilibrium much earlier than the energy containing portion of the spectra. The development of the energy containing portion lags the wind growth. Since the value of $D_{P}$ is controlled by the total wave energy 
while the value of $E_{P}$ is controlled by $u_{*}$, we therefore expect that $D_{P}$ will lag $E_{P}$ as well.

\subsection{Relationship to ambient noise.}

Laboratory experiments [e.g., Melville et al (1988), Loewen \& Melville (1991a)] show that the sound generated by breaking waves is proportional to the total energy dissipated by the event over a limited range of parameters. Melville (1992) suggested that this proportionality is due to the fact that the dissipation and ambient noise generation are coupled by the air entrainment process. The correlation between sound generation and dissipation by breaking waves in the laboratory suggests that sound may be used to scale the frequency and intensity of wave breaking in the field. Although models (e.g., Farmer \& Vagle, 1988) have suggested that the frequency and intensity of wave breaking in the vicinity of a hydrophone is proportional to the ambient noise level, it has yet to be demonstrated by field measurements that dissipation by breaking waves correlates with the ambient noise in the field.

Figure 5.6 shows plots of $\mathrm{D}_{\mathrm{H}}$ versus the ambient noise at $4.3,8.0,14.0$ and $2.5-14 \mathrm{kHz}$ $(\Sigma)$. Similar plots showing the relationship between ambient noise and the other dissipation estimates are given in the succeeding figures: Figure $5.7\left(\mathrm{E}_{\mathrm{H}}\right)$, Figure 5.8 $\left(D_{\mathrm{P}}\right)$ and Figure $5.9\left(\mathrm{E}_{\mathrm{P}}\right)$. The plots show a good correlation between ambient noise and the dissipation except for $\mathrm{N}(14 \mathrm{kHz})$. As we pointed out earlier, field measurements [e.g., Farmer \& Lemon (1984), Vagle et al (1990)] suggest that sound absorption by bubbles generated by breaking waves can significantly alter noise levels above $8 \mathrm{kHz}$. For this reason, we will confine our discussion in this section to the three other noise frequencies given in the figures. 

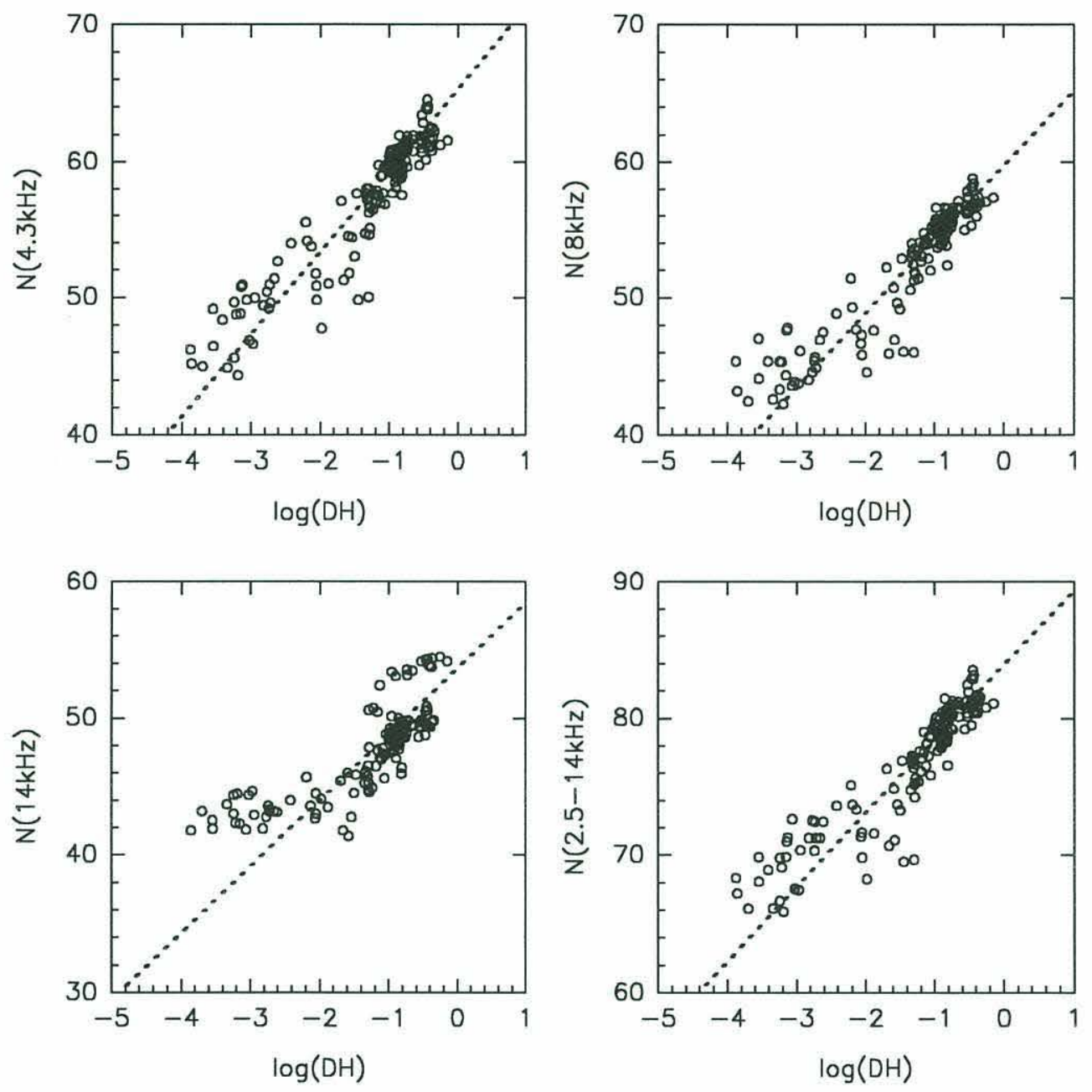

Figure 5.6. Plots of the $\mathrm{KHH}$ dissipation $\mathrm{D}_{\mathrm{H}}$ versus ambient noise at selected frequencies. The dashed line indicates the regression computed from the data (see Table 5.1 for the coefficients). 

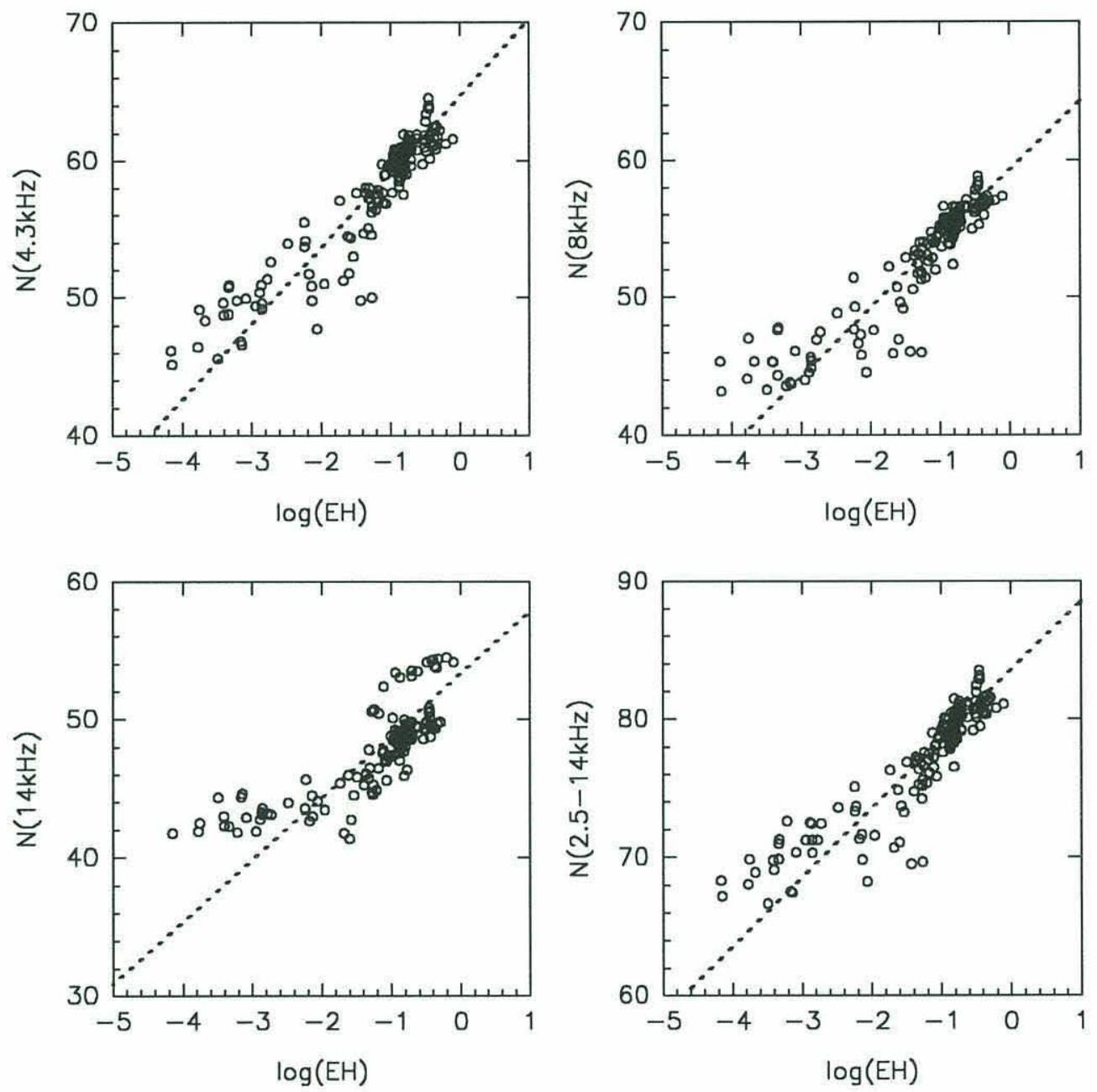

Figure 5.7. Plots of the reduced $\mathrm{KHH}$ dissipation $\mathrm{E}_{\mathrm{H}}$ versus ambient noise at selected frequencies. The dashed line indicates the regression computed from the data (see Table 5.1 for the coefficients). 

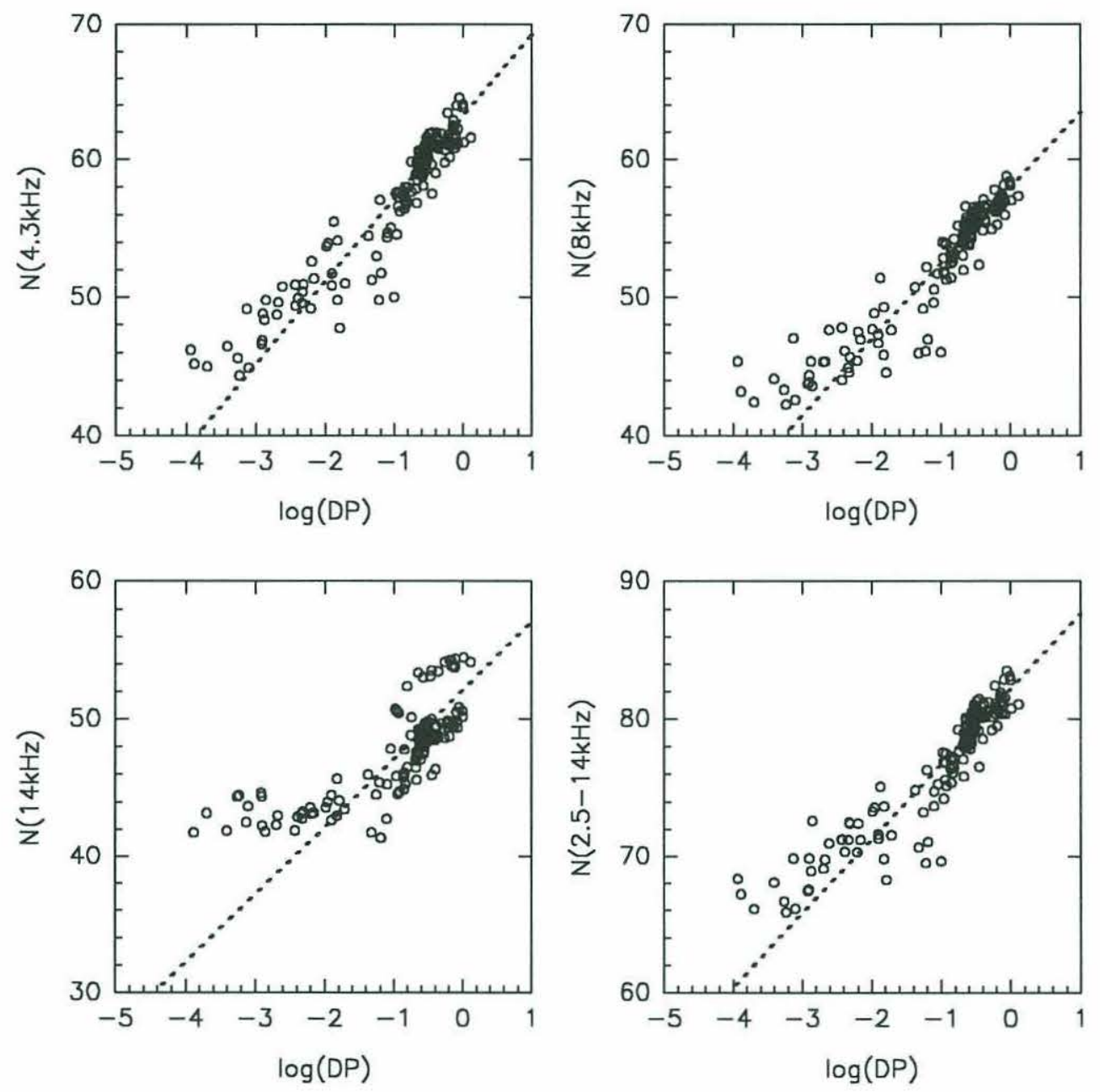

Figure 5.8. Plots of the Phillips (1985) dissipation $D_{P}$ versus ambient noise at selected frequencies. The dashed line indicates the regression computed from the data (see Table 5.1 for the coefficients). 

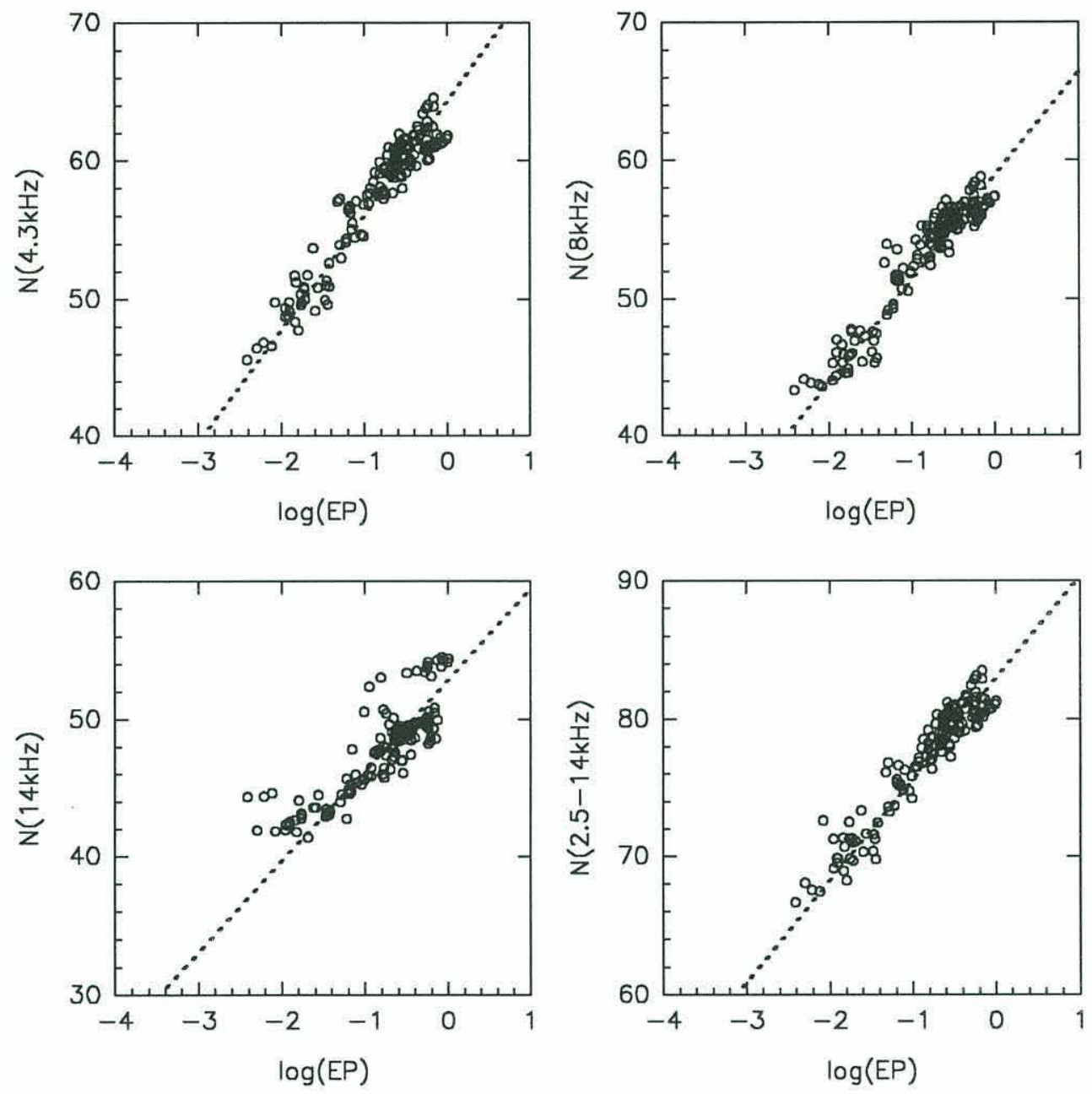

Figure 5.9. Plots of the Phillips (1985) dissipation $\mathrm{E}_{\mathrm{P}}$ versus ambient noise at selected frequencies. The dashed line indicates the regression computed from the data (see Table 5.1 for the coefficients). 
Table 5.2. Coefficients for the ambient noise and dissipation expression and the equivalent power laws computed from NOBS data.

$$
\log \mathrm{D}=\mathrm{m}(\mathrm{f}) \mathrm{N}(\mathrm{f})+\mathrm{n}(\mathrm{f}) \quad \frac{\mathrm{p}^{2}}{\mathrm{p}_{\mathrm{ref}}^{2}}=m \mathrm{D}^{n}
$$

1. $\mathrm{D}_{\mathrm{H}}$

\begin{tabular}{|c|c|c|c|c|c|}
\hline $\mathrm{f}(\mathrm{kHz})$ & $C_{x y}$ & $\mathrm{~m}$ & $\mathrm{n}$ & $10^{-6} m$ & $n$ \\
\hline 4.3 & 0.93 & 0.167 & -10.90 & 3.36 & 0.60 \\
\hline 8.0 & 0.93 & 0.184 & -10.99 & 0.94 & 0.54 \\
\hline 14.0 & 0.81 & 0.207 & -11.10 & 0.23 & 0.48 \\
\hline$\Sigma$ & 0.92 & 0.185 & -15.52 & 245 & 0.54 \\
\hline
\end{tabular}

2. $\mathrm{E}_{\mathrm{H}}$

\begin{tabular}{|c|c|c|c|c|c|}
\hline 4.3 & 0.93 & 0.181 & -11.71 & 2.95 & 0.55 \\
\hline 8.0 & 0.93 & 0.199 & -11.80 & 0.85 & 0.50 \\
\hline 14.0 & 0.81 & 0.223 & -11.89 & 0.21 & 0.45 \\
\hline$\Sigma$ & 0.91 & 0.200 & -16.71 & 226 & 0.50 \\
\hline
\end{tabular}

3. $\mathrm{D}_{\mathrm{P}}$

\begin{tabular}{|c|c|c|c|c|c|}
\hline 4.3 & 0.95 & 0.166 & -10.49 & 2.09 & 0.60 \\
\hline 8.0 & 0.94 & 0.182 & -10.55 & 0.63 & 0.55 \\
\hline 14.0 & 0.79 & 0.201 & -10.47 & 0.16 & 0.50 \\
\hline$\Sigma$ & 0.93 & 0.183 & -15.04 & 165 & 0.55 \\
\hline
\end{tabular}

4. $E_{P}$

\begin{tabular}{|c|c|c|c|c|c|}
\hline 4.3 & 0.95 & 0.121 & -7.78 & 2.69 & 0.83 \\
\hline 8.0 & 0.93 & 0.133 & -7.83 & 0.77 & 0.75 \\
\hline 14.0 & 0.79 & 0.152 & -8.03 & 0.19 & 0.66 \\
\hline$\Sigma$ & 0.94 & 0.136 & -11.28 & 197 & 0.74 \\
\hline
\end{tabular}




\section{Chapter 5}

We also see from Figure 5.5 - 5.7 that the distribution of the data around the regression lines appear to be qualitatively similar for $E_{H}, D_{H}$ and $D_{P}$. This should be evident from the dissipation time series given in Figures 5.1a and 5.1c. In Figure 5.1a, we see that the $\mathrm{D}_{\mathrm{H}}$ and $\mathrm{E}_{\mathrm{H}}$ time series are nearly indistinguishable. In Figure 5.1c, the $\mathrm{D}_{\mathrm{H}}$ and $\mathrm{E}_{\mathrm{H}}$ time series also look very similar with the difference between the values of $\log \mathrm{D}_{\mathrm{P}}$ and $\log \mathrm{D}_{\mathrm{H}}$ nearly constant.

We also see that the dissipation data where $\log \mathrm{D}>-1.5$ show considerably less scatter than data where $\log \mathrm{D}<-1.5$. This is partly due to the distribution of the available data. In Section 4.6.3, we saw that the wind and wave conditions were quasi-steady with the wind speed and dissipation constant for approximately half of the observation period. As a result, the low $\mathrm{N}$ data is sparse and the data distribution is biased towards the higher winds speeds and dissipation values.

It is important to point out that except for $\mathrm{E}_{\mathrm{P}}$, the dissipation estimates were all computed directly from wave field parameters. $\mathrm{E}_{\mathrm{H}}$ was computed from the variance of the wind wave field $a_{w}{ }^{2}$ and the mean frequency $\bar{f}_{w}$ while $\mathrm{D}_{\mathrm{H}}$ and $\mathrm{D}_{\mathrm{P}}$ were computed from the measured spectra. In Section 1.2, we described how previous field measurements have found that the ambient noise $\mathrm{N}$ and the log of the root mean square wave amplitude $a$ are poorly correlated. The correlation coefficient between the two parameters for the NOBS data is 0.67 which is comparable to the value obtained by Perrone (1969) and by Farmer \& Lemon (1984).

Our analysis in Section 4.6.2 showed that by filtering out swell and comparing $\mathrm{N}$ with the RMS wave height of the wind wave components, we can improve the relationship between the two (Figure 4.13). By neglecting the contribution of swell, we increased the correlation coefficient between $\mathrm{N}$ and $\log a_{w}$ to 0.87 . A similar value for the 
correlation coefficient was obtained between $\mathrm{N}$ and $\log s$ (the RMS wave slope). While correlation coefficients for $\mathrm{N}$ vs. $\log a_{w}$ is significantly higher than the values for the correlation coefficient for $\mathrm{N}$ vs. $\log a$, it is still significantly less than the correlation coefficient of the $\mathrm{N}$ vs. $\log \mathrm{U}$ relationship (correlation coefficient $=0.94$ ).

Section 4.6.3 showed that comparisons between wind and wave parameters and $\mathrm{N}$ during steady conditions are not meaningful since the dynamic range of the values during this period is small. Hence, we omit comparisons between $\mathrm{N}$ and log dissipation during steady conditions. Section 4.6 .3 also showed that the results for the unsteady cases are similar to those for the combined steady and unsteady cases. Table 5.2 gives the correlation coefficients between $\mathrm{N}$ and the different dissipation estimates. We see from this table that the relationship between log dissipation and $\mathrm{N}$ give correlation coefficients (0.93-0.95) that are comparable to that between log $\mathrm{U}$ and $\mathrm{N}$.

Table 5.2 also compiles the different regression equations for the various dissipation versus ambient noise plots. For purposes of this work, we adopted the form of the WOTAN equation to relate the dissipation $\mathrm{D}$ to ambient noise.

$$
\log \mathrm{D}=\mathrm{m}(\mathrm{f}) \mathrm{N}(\mathrm{f})+\mathrm{n}(\mathrm{f})
$$

The coefficients $\mathrm{m}$ and $\mathrm{n}$ in Table 5.2 were computed using the orthogonal linear regression formula (Casella, 1990, p. 586). The regression lines are shown as dashed lines in each plot in Figures 5.6 - 5.9.

Equation 5.3 is equivalent to a power law expression between the sound pressure and surface wave dissipation. We can therefore express it in terms of the equation 


\section{Chapter 5}

$$
\frac{\mathrm{p}^{2}}{\mathrm{p}_{\mathrm{ref}}^{2}}=m \mathrm{D}^{n}
$$

where $m=10^{-\mathrm{n}} / 10 \mathrm{~m}$ and $n=1 /(10 \mathrm{~m})$. Our computations suggest that the power law dependence between the ambient noise pressure level and dissipation is

$$
\mathrm{p}^{2} \sim \mathrm{D}^{0.5-0.6}
$$

for the estimates computed from wave field characteristics $\left(\mathrm{D}_{\mathrm{H}}, \mathrm{E}_{\mathrm{H}}\right.$ and $\left.\mathrm{D}_{\mathrm{P}}\right)$, and a slightly steeper power law dependence

$$
\mathrm{p}^{2} \sim \mathrm{D}^{0.8}
$$

when the dissipation is computed from $\mathrm{u}_{*}\left(\mathrm{E}_{\mathrm{P}}\right)$.

Despite these differences, our results show that the different models give dissipation estimates that are comparable. They also show that dissipation correlates well with ambient noise in the field. This is consistent with the results of the laboratory experiments we reviewed in Section 1.4 which suggest that the wave energy dissipated and the acoustic energy generated by a breaking wave are both proportional to the strength of breaking. Since surface wave dissipation and the generation of underwater sound are coupled together by the air entrainment process associated with breaking waves, then the two correlated well in the laboratory. Our results show that the correlation between the two are observed in the field as well. 


\section{DISCUSSION AND CONCLUSIONS}

In the review in Chapter 1, we discussed how several decades of research have shown that the wind speed shows a very strong correlation with ambient noise $\mathrm{N}$ in the Knudsen range. The research in the last decade or so have taken advantage of this in developing Weather Observations Through Ambient Noise (WOTAN), a method which uses the level of ambient noise at preselected frequencies to estimate the wind speed $\mathrm{U}$ using the linear relationship between $\mathrm{N}$ and $\log \mathrm{U}$.

In contrast, research relating the ambient noise level to parameters of the wave field have been few and largely unsuccessful. The available measurements have shown that $\mathrm{N}$ and the significant wave height (or the root mean square wave amplitude $a$ ) are poorly correlated. No attempt has been made to explore this subject further nor have attempts been made to establish a mathematical relationship between the two parameters.

Measurement have shown that breaking waves in the field generate sound as low as $30 \mathrm{~Hz}$ and up to $20 \mathrm{kHz}$. Although acoustic field measurements below $500 \mathrm{~Hz}$ have been known to correlate with wind speed, the influence of shipping, industrial and other man-made noise can contaminate measurements at these frequencies.

Laboratory measurements have shown that the major sources of sound from breaking waves are due to bubble oscillations during creation, coalescence and breakup. These mechanisms dominate the sound frequencies above $1 \mathrm{kHz}$ and perhaps as low as a few hundred Hertz. At the lowest frequencies of wind dependent noise of the order of tens of Hertz, there is evidence that the sound is due to the collective oscillations of bubble clouds. 


\section{Chapter 6}

If it is indeed wave breaking that is responsible for the noise in the wind dependent frequency range, it is then surprising that the characteristics of the wave field and the ambient underwater sound have appeared to be poorly correlated. Although wave breaking is ultimately driven by the wind, the wave breaking process is a direct product of the characteristics of the wave field. This apparent contradiction has yet to be resolved.

We believe that the key to understanding this relationship is to relate $\mathrm{N}$ to wave parameters that are either directly responsible for the occurrence of wave breaking or are direct manifestations of the wave breaking process. The various laboratory measurements of wave breaking by Melville and his coworkers have provided us with some clues.

Laboratory experiments of mechanically generated breaking waves suggest that a packet consisting of focused short and long waves can produce breaking waves whose gross characteristics can be consistently reproduced. The packets can be characterized by a slope parameter $s_{p}$ whose value is a measure of the aggregate steepness of the components of the wave packet. These experiments found that the magnitude of $s_{p}$ correlates with the magnitude of several important characteristics of breaking wave including, loss of excess momentum flux, mixing, initial volume of air entrained, wave energy dissipation and the acoustic energy generated by the event.

In particular, we focus on the recent studies by Melville et al (1988) and Loewen \& Melville (1991a) which show that the energy dissipated by a breaking wave is proportional to the volume of air entrained and the sound generated by the event. The results of the work of Lamarre \& Melville (1991) on the evolution of the bubble cloud generated by the breaking wave suggest that a large fraction (up to 50\%) of the energy 
dissipated is due to the work done against buoyancy in entraining the bubble plume. At the same time, creating air bubbles, forcing them and the bubble cloud itself leads to the conversion of mechanical energy into acoustic energy. This process is clearly not very efficient since only a very small fraction of the order of $10^{-8}$ (Loewen \& Melville, 1991a) of the mechanical energy is actually converted to sound.

Nevertheless, both dissipation and sound generation in breaking waves are coupled together by the air entrainment process and we believe that this is the reason why the two correlate well.

The laboratory experiments clearly suggest that level of dissipation could be used as an effective variable in parameterizing the occurrence of wave breaking in the field. In principle, one can compute the total dissipation in the wave field by summing up the total energy dissipated by all the individual events. In practice, this is difficult to undertake since not only is it hard to estimate how much energy each individual event dissipates, it is also difficult to come up with a reliable and effective method for performing areal surveys of the occurrence and intensity of breaking waves in the field using commonly available instruments and techniques.

Alternatively, we can indirectly estimate the total dissipation by examining how energy is added, dissipated and redistributed in the wave field. This process is described mathematically by the energy transfer equation (Equation 1.11) which expresses the changes in the wave energy spectrum as a sum of the wind energy input $w(\mathbf{k})$, the nonlinear flux of energy from the higher frequencies to the lower frequencies $\nabla_{\mathbf{k}} \cdot \mathbf{T}(\mathbf{k})$, and the dissipation of the wave energy $\mathrm{d}(\mathbf{k})$.

The characteristics of the spectral dissipation $\mathrm{d}(\mathbf{k})$ are at present not very well understood. In Chapter 2, we described two theories on how $\mathrm{d}(\mathbf{k})$ can be computed 


\section{Chapter 6}

from the wave spectrum. Using heuristic arguments on the influence of nonlinear but localized wave breaking on the wave field, Hasselmann (1974), proposed that

$$
\mathrm{d}_{\mathrm{H}}(\mathbf{k}) \sim \omega^{2} \Psi(\mathbf{k}) .
$$

The coefficient of this relationship was later obtained by Komen et al (1984) from numerical simulations of the equilibrium balance between $\mathrm{d}(\mathbf{k}), \mathrm{w}(\mathbf{k})$ and $\nabla_{\mathbf{k}} \cdot \mathrm{T}(\mathbf{k})$. Although subsequent numerical wave evolution models [e.g., SWAMP (1985), WAMDIG (1988)] have attempted to incorporate more parameters and to enhance the stability of $\mathrm{d}_{\mathrm{H}}$, the basic assumptions Hasselmann (1974) made in deriving the form of $\mathrm{d}_{\mathrm{H}}$ are still used in many of the current wave evolution models.

To date, the most rational approach to the estimate of spectral dissipation is due to Phillips (1985) who derived an analytical expression for dissipation based essentially on two major assumptions on the characteristics of the equilibrium range above the wind wave spectral peak: (a) that the magnitude of $d(\mathbf{k}), w(\mathbf{k})$ and $\nabla_{\mathbf{k}} \cdot T(\mathbf{k})$ are comparable, and (b) that compared to the time and spatial scales of evolution of the wave spectrum, the equilibrium range wave components are quasi-steady [or that the sum of $d(\mathbf{k}), w(\mathbf{k})$ and $\nabla_{\mathbf{k}} \cdot T(\mathbf{k})$ is zero]. From these assumptions, Phillips (1985) derived an expression for $\mathrm{d}(\mathbf{k})$ and showed that in the equilibrium range, the wind wave spectrum

$$
\Phi_{\mathrm{E}}=\alpha \mathrm{u}_{*} \mathrm{~g} \omega^{-4} .
$$

While $d_{H}$ and $d_{P}$ are both based on assumptions of equilibrium among the source terms, their major differences arise from the different assumptions on the influence of the wave spectrum on $\mathrm{d}(\mathbf{k})$ and the differences on the assumed forms of the other 


\section{Chapter 6}

source terms in the energy transfer equation. Hence, the use of either model to estimate dissipation requires that we first examine the differences between the two and how their values compare using real wind and wave data.

In Chapter 3, we described an experiment in the open ocean $130 \mathrm{~km}$ off the coast of Oregon where we made simultaneous measurements of the wind speed, the ambient noise and the wave height spectrum using an array of wire wave gauges from the research platform Flip. The hydrophone data was sampled at $51.2 \mathrm{kHz}$ using a directional hydrophone. The power spectrum of the data was computed in real time by a DSP board in a PC and stored on optical disks. By processing the data in situ, we significantly reduced the storage and post-experiment processing requirements to manageable levels. All other instruments used in our experiment were sampled by Robert Pinkel and Jerome Smith of the Upper Ocean Physics Group, Scripps Institution of Oceanography. From this data, directional wave spectra were computed using the maximum likelihood method from the wave height measurements of the four wire wave gauges. The time series of the root mean square wave slope $s$ was also computed.

We found (Chapter 4) that due to the various noise sources, the higher frequency portion of the ambient noise data was unusable. However, most of the acoustic data at frequencies below $14 \mathrm{~Hz}$ appear to be reasonably free of noise once the sources of noise were characterized and eliminated. For our purposes, the comparisons with ambient noise of different frequencies with wind and wave parameters give qualitatively comparable results so unless indicated, this section will discuss the $\mathrm{N}(4.3 \mathrm{kHz})$ results. In general, we found that the wind versus ambient noise relationships from our measurements are consistent with those reported earlier. Our measurements give (Figures 4.9 and 4.10 ) 


\section{Chapter 6}

$$
\begin{aligned}
& \mathrm{p}^{2} \sim \mathrm{U}_{10}^{3} \\
& \mathrm{p}^{2} \sim \mathrm{u}_{*}^{2.3} .
\end{aligned}
$$

The more novel aspects of this study however are in the comparisons between the wave field and the ambient noise. As we mentioned earlier, we found it surprising that there seemed to be very little in the literature that sought to establish and define relationships between these two. The few experiments that were reported show the root mean square wave amplitude $a$ and $\mathrm{N}$ to be poorly correlated.

Penhallow \& Dietz (1964) suggested that the changes in the wind speed, $a$ and ambient noise are better correlated under steady wind conditions. When the wind was increasing or decreasing, $\mathrm{N}$ and $\mathrm{U}$ were still reasonably well-correlated but $\mathrm{N}$ and $a$ were not. In their paper, they showed a correlation coefficient of 0.90 between $\mathrm{N}$ and $a$ under steady winds and 0.42 when the wind speed is increasing. We found results (Section 4.6.3) that were the opposite of those they reported. Our results showed that the data correlated better during unsteady cases (Figure 4.5) than during steady wind conditions. With our data, the reason is clear from Figure 4.18. In that figure, we see that the scatter in the data is relatively constant under both categories but the range in $\mathrm{U}_{10}, a$ and $\mathrm{N}$ values is larger for unsteady conditions. For steady winds, the data is clustered together thus making the data appear uncorrelated.

It is difficult to make meaningful comparisons between the Penhallow \& Dietz (1964) data and ours since they showed no plots of the data in their paper. However, the fact that the correlation coefficients for the unsteady data set and the complete data set do not differ significantly (less than \pm 0.02 ) [see Table 4.5] suggests that our analysis of the data is robust and is not sensitive to the quantity of the data we analyzed. 


\section{Chapter 6}

We found that while $a$ and $\mathrm{N}$ do not correlate well, we can improve the correlation between the two if we filter out the swell components from the wave elevation time series. When this is done, the relationship between $\mathrm{N}$ and $a_{w}$ is improved significantly (Figure 4.13). Our results show that

$$
\mathrm{p}^{2} \sim a_{w}^{2.2}
$$

The effect of swell on the sea surface variance $a^{2}$ is disproportionate to its effect on the frequency of wave breaking and consequently, to N. Since swell is generated outside the region, its properties are not a direct result of the local wind-wave conditions. On a calm day, we can get high $a$ values if there is significant swell. By filtering this out and considering only the wave field components that are generated locally, then the $a_{w}$ versus $\mathrm{N}$ relation is improved.

In addition, we note that the spectral energy in the wave height frequency spectrum $\Phi(f)$ is maximum at the wind wave spectral peak and decays very rapidly at higher frequencies (frequency slope $\mathrm{f}^{-4}$ up to approximately $1 \mathrm{~Hz}$ and $\mathrm{f}^{-7}$ above that). Hence, the change in the value of $a_{w}$ is dominated by the changes in the energy of the wave frequencies near the spectral peak. However, we found that, as the Snyder et al (1981) and the Plant (1982) wind input formulas suggest, the higher frequencies are more responsive to changes in the wind speed and direction and consequently, to changes in $\mathrm{N}$.

This is reflected in how the directional wave spectrum changes. In Figures 4.7 and 4.8, we saw that when the wind began to pick up after it changed direction by $180^{\circ}$, the wave energy in the new direction was first seen at the higher frequency wave 
components $(\mathrm{f} \sim 1 \mathrm{~Hz}$ ). Eventually, the energy in the lower frequency components began increasing until the directional distribution of the wave energy spectrum regained its original form before the change in the wind direction.

In Figure 4.14, we also observed that although the relationship between the log of the spectral level of the wind wave frequencies above the peak is linear with $\mathrm{N}$, the higher frequency wave components do not exhibit the increased scatter in the data at low $\mathrm{N}$ and $\mathrm{U}_{10}$ values. Clearly, the key to understanding the relationship between the ambient noise levels and the wave spectrum is in the higher frequencies. The mechanisms for sound generation are more closely related to these than to $a$ whose value is determined more by the lower frequency components.

As we discussed earlier in this chapter, experiments on laboratory generated breaking waves [(Rapp \& Melville, 1990), (Melville et al, 1988), (Loewen \& Melville, 1991a)] showed that the steepness of the wave packet correlate with the magnitude of many of the variables associated with wave breaking. If we argue that the laboratory packet steepness can be considered as the analog of the steepness of the wave field, then we can argue that the RMS wave slope $s$ (or some measure of wave field steepness) correlates with the characteristics of wave breaking in the field as well. We can therefore expect that, similar to the laboratory results, wave slope correlates with ambient noise as well. When we compared $s$ with $N($ Section 4.6.2), we found that log $s$ correlates with $\mathrm{N}$ and that the relationship between the two can be described by a power law where

$$
\mathrm{p}^{2} \sim s^{3.4} .
$$




\section{Chapter 6}

As we mentioned earlier, one of the key objectives of this work is to examine the spectral dissipation models and see how they relate to wave field parameters and $\mathrm{N}$. While the dissipation models based on Hasselmann's (1974) assumptions are currently more widely used in the development of numerical wave evolution models, the dissipation estimates based on Phillips (1985) appear to be grounded on a more rational basis. However, although there are clearly differences between the two, as we saw in Chapter 5, they both give estimates that are comparable to one another. In general, the Phillips (1985) estimate is approximately twice that given by Komen et al (1984). Given that the dynamic range of $\mathrm{D}$ is at least 4 orders of magnitude over this data set, this is reasonably good agreement.

The coefficients of the $\mathrm{KHH}$ dissipation expression $\mathrm{d}_{\mathrm{H}}(\mathbf{k})$ were obtained by tuning $\mathrm{d}_{\mathrm{H}}(\mathbf{k})$ with the wind input term $\mathrm{w}(\mathbf{k})$ and the nonlinear spectral flux term $\nabla_{\mathbf{k}} \cdot \mathbf{T}(\mathbf{k})$ in the energy transfer equation (Equation 1.11) to reproduce observed fetch-limited wave growth. On the other hand, Phillips' (1985) dissipation expression $\mathrm{d}_{\mathrm{P}}(\mathbf{k})$ was obtained by assuming an equilibrium balance between $d_{P}(\mathbf{k}), \nabla_{\mathbf{k}} \cdot T(\mathbf{k})$, and $w(\mathbf{k})$. The form of the equilibrium wave spectrum $\Phi_{\mathrm{E}}(\omega)=\alpha \mathrm{u}_{*} \mathrm{~g} \omega^{-4}$ arises as a consequence of Phillips' (1985) choices for the analytical expressions of $\nabla_{\mathbf{k}} \cdot \mathbf{T}(\mathbf{k}), \mathrm{w}(\mathbf{k})$ and $\mathrm{d}(\mathbf{k})$. Comparisons between the model $\Phi_{\mathrm{E}}(\omega)$ expression and measured wave spectra $\Phi(\omega)$. [Figure 4.21] show that $\Phi_{\mathrm{E}}(\omega)$ gives magnitude and slope values similar to those of $\Phi(\omega)$.

Phillips (1985) also argued that the presence of wind drift $\mathrm{c}_{\mathrm{q}} \approx \mathrm{u} *$ inhibits the formation of high frequency waves and therefore sets an upper limit to $\Phi_{E}(\omega)$. He speculated that this upper frequency limit $\omega_{1} \approx \mathrm{rg} / \mathrm{u}_{*} \approx 0.4 \mathrm{~g} / \mathrm{u}_{*}$. While the observed wave height spectrum $\Phi(\omega)$ does show an upper limit to the $\omega^{-4}$ frequency slope, its magnitude is between $0.1-0.2 \mathrm{~g} / \mathrm{u}_{*}$, approximately one third of the value predicted by Phillips (1985). It is possible that either the true wind drift values are smaller than 
Phillips (1985) estimated or that some other mechanism inhibits the formation of waves at frequencies lower than the frequencies wind drift would affect.

The major differences between $E_{H}$ and $E_{P}$ arise because Phillips (1985) uses $\Phi_{E}(\omega)$ instead of $\Phi(\omega)$ to compute the total dissipation. Although we showed that the differences between the two spectra are generally small, these differences are magnified because of the cubic dependence of $E_{P}$ on $\Phi(\omega)$.

In addition, because $\mathrm{E}_{\mathrm{P}}$ uses $\Phi_{\mathrm{E}}(\omega)$, the non-constant variables which control the value of $E_{\mathrm{P}}$ are $\mathrm{u}_{*}$ and the phase speed of the peak frequency $c_{\mathrm{p}}$. From Equation 2.17, we note that

$$
\mathrm{E}_{\mathrm{P}} \sim \mathrm{u}_{*}^{3} \ln \left[\mathrm{r}\left(\mathrm{c}_{\mathrm{p}} / \mathrm{u}_{*}\right)^{2}\right]
$$

The dependence on $u_{*}$ is cubic while the influence of the wave field through the natural log of the wave age parameter is substantially weaker.

On the other hand, the non-constant variables in the KHH estimate are the PiersonMoskowitz steepness parameter $\bar{\alpha}=a_{w}^{2} \bar{\omega}_{w}^{4} / g^{2}$, the RMS wind wave amplitude $a_{w}$, and the mean frequency $\bar{\omega}_{w}$. Rearranging Equation 2.27 and evaluating $\bar{\alpha}$, it is easy to show that

$$
\mathrm{E}_{\mathrm{H}} \sim a_{w}^{6} \bar{\omega}_{w}^{9}
$$

indicating that, using the $\mathrm{KHH}$ model, the total dissipation of the wave field can be computed from integral wind wave field characteristics. 
It is also evident from Equations 6.1 and 6.2 why, as seen in Figure 5.1d, changes in $\mathrm{E}_{\mathrm{H}}$ can lag changes in $\mathrm{E}_{\mathrm{P}}$. The values of $a_{w}$ and $\bar{\omega}_{w}$ are controlled by the energycontaining portion of the wind wave spectrum. However, as we saw in Figures 4.7 and 4.8, u* more directly influences the higher frequencies which achieve full development at substantially lower energy levels than the spectral peak.

Consequently, these frequencies have a lesser influence on the values of $a_{w}$ and $\bar{\omega}_{w}$.

Our results show that, consistent with the laboratory measurements of breaking waves by Melville et al (1988) and Loewen \& Melville (1991a), the ambient noise and the different total dissipation estimates correlate well in the field. The relationship between dissipation and ambient noise was empirically shown to be

$$
\mathrm{p}^{2} \sim \mathrm{E}_{\mathrm{P}}^{0.8}
$$

for the Phillips (1985) model and

$$
\mathrm{p}^{2} \sim \mathrm{E}_{\mathrm{H}}^{0.6}
$$

for the KHH model.

In conclusion, scientists have long recognized the importance of understanding the physical processes in the interaction between the atmosphere and the ocean. These processes play an important role in determining climate, the general circulation of the ocean and the atmosphere, the formation of water masses, and the transfer of gas and moisture between the two media. One of the key issues in describing these processes is our ability to characterize the evolution of the wave field and how the wave field 
affects the air-sea boundary conditions and fluxes. Although a lot of research effort has been made to promote our understanding of these processes, one of the major obstacles in achieving this objective is our ability to quantify and characterize the breaking wave dissipation source functions. Our research shows that the relationship between sound and dissipation in breaking waves may prove to be an important tool in quantifying dissipation in the field. 


\section{REFERENCES}

Agrawal, Y.C., et. al. 1992 Enhanced dissipation of kinetic energy beneath surface waves. Nature. 359, 219-220.

Axelrod, E.H., B.A. Schoomer and W.A. Von Winkle 1965 Vertical directionality of ambient noise in the deep ocean at a site near Bermuda. J. Acoust. Soc. Am. 37, 77-83.

Banner, M.L. and D.H. Cato 1988 Physical mechanisms of noise generation by breaking waves - a laboratory study. In Sea Surface Sound. B.R. Kerman, ed. Kluwer Academic, Dordrecht, Netherlands, 429-436.

Banner, M.L. and O.M. Phillips 1974 On the incipient breaking of small scale waves. J. Fluid Mech. 65, 647-656.

Bendat, J.S and A.G Piersol 1986 Random Data: Analysis and Measurement Procedures. John Wiley \& Sons, Inc. Canada.

Bronson, E.D. and L.R. Glosten 1985 FLIP: FLoating Instrument Platform. SIO REFERENCE $85-21$. Revision of previous SIO Reference $73-30$ by C.B. Bishop and D.O. Efird.

Burgess, A.S. and D.J. Kewley 1983 Wind-generated surface noise source levels in deep water east of Australia. J. Acoust. Soc. Am. 73, 201.

Capon, J. 1969 High-resolution frequency-wavenumber spectrum analysis. Proc. IEEE $57,1408-1418$. 
Carey, W.M. and D. Browning 1988 Low frequency ambient noise: measurements and theory. In Sea Surface Sound. B.R. Kerman, ed. Kluwer Academic, Dordrecht, Netherlands, 361-376.

Carey, W.M. and J.W. Fitzgerald 1992 Low frequency noise from breaking waves. In Natural Physical Sources of Underwater Sound. B.R. Kerman, ed. Kluwer Academic, Dordrecht, Netherlands, 277-304.

Casella, G. 1990 Statistical Inference. Wadsworth and Brooks, Pacific Grove, CA.

Cato, D.H. 1976 Ambient sea noise in waters near Australia. J. Acoust. Soc. Am. 60, 320328.

Crouch, W.W. and P.J. Burt 1972 The logarithmic dependence of surface-generated ambient sea-noise spectrum level on wind speed. J.Acoust. Soc. Am. 51, 1066-1072.

Crowther, P.A. and A. Hansla 1993 The lifetimes, velocities and probable origin of sonic and ultrasonic noise sources on the sea surface. In Natural Physical Sources of Underwater Sound. B.R. Kerman, ed. Kluwer Academic, Dordrecht, Netherlands, 379-392.

Ding, L. 1993 Acoustical Studies of Breaking Surface Waves in the Open Ocean. PhD Thesis. The University of Victoria p. 100.

Ding, L. and D.M. Farmer 1992 A signal processing scheme for passive acoustical mapping of breaking surface waves. J. Atmos. E Oceanic Tech. 9, 484-494. 
Dobson, F., W. Perrie and B. Toulany 1989 On the deep-water fetch laws for windgenerated surface gravity waves. Atmos.-Ocean. 27, 210-236.

Donelan, M.A., J. Hamilton and W.H. Hui 1984 Directional spectra of wind-generated waves. Phil. Trans. R. Soc. Lond. A 315, 509-562.

Donelan, M., M. Skafel, H. Graber, P. Liu, D. Schwab and S. Venkatesh 1992 On the growth of wind-generated waves. Atmos.-Ocean. 30, 457-478.

Duncan, J.H. 1981 An investigation of breaking waves produced by a towed hydrofoil. Proc. Roy. Soc. Lond. A 377, 331-348.

Dyer, Ira 1989 Course notes in 13.851 Fundamentals and Applications of Underwater Sound.

Evans, D.L., D.R. Watts, D. Halpern and S. Bourassa 1984 Ocean winds measured from the seafloor. J. Geophys. Res. 89, 3457-3461.

Farmer, D.M. and D.D. Lemon 1984 The influence of bubbles on ambient noise in the ocean at high wind speeds. J. Phys. Oceanogr. 14, 1762-1778.

Farmer, D.M. and S. Vagle 1988 On the determination of breaking surface wave distributions using ambient sound. J. Geophys. Res. 93, 3457-3461.

Farmer, D.M. and S. Vagle 1989 Wave propagation of ambient sound in the oceansurface bubble layer. J. Acoust. Soc. Am. 86, 1897-1908. 
Fitzpatrick, H.M. and M. Strasberg 1956 Hydrodynamic sources of sound in Symposium on Naval Hydrodynamics. 24-28 Sept. 1956, Washington, DC, Pub. 5.5, NAS-NRC, 241280.

Forristall, G.Z. 1981 Measurements of a saturated range in ocean wave spectra. J. Geophys. Res. 86, 8075-8084.

Fox, M.J.H. 1976 On the nonlinear transfer of energy in the peak of a gravity wave spectrum II. Proc. R. Soc. London A 348, 467-483.

Franz, G.J. 1959 Splashes as sources of sound in liquids. J. Acoust. Soc. Am. 31, 10801096.

Furduev, A.V. 1966 Underwater surface cavitation as a source of noise in the ocean. Atmos. Oceanic Phys. 2, 314-320.

Hasselmann, K. 1962 On the nonlinear transfer of energy in the peak of a gravity wave spectrum. Part 1. J. Fluid Mech. 12, 481-500.

Hasselmann, K. 1963 On the nonlinear transfer of energy in the peak of a gravity wave spectrum. Parts 2 and 3. J. Fluid Mech. 15, 273-281, 385-398.

Hasselmann, K. 1974 On the spectral dissipation of ocean waves due to whitecapping. Boundary-Layer Met. 6, 107-127.

Hill, W. 1984 A generation of WOTAN underwater wind-recording instruments. Proc. IEEE OCEANS '84, 31-36. 
Holthuijsen, L.H. and T.H.C. Herbers 1986 Statistics of breaking waves observed as whitecaps in the open sea. J. Phys. Oceanogr. 16, 290-297.

Jeffreys, E.R., G.T. Wareham, N.A. Ramsden and M.J. Platts 1981 Measuring directional spectra with the MLM. In Directional Wave Spectra Applications, Proceedings of the conference in Berkeley, CA, Sept. 14-16, 1981, 203-219.

Jessup, Andrew T. 1990 Detection and Characterization of Deep Water Wave Breaking Using Moderate Incidence Angle Microwave Backscatter from the Sea Surface. PhD Thesis, Massachusetts Institute of Technology, Cambridge, MA.

Katsaros, K.B. and S.S. Ataktürk 1991 Dependence of wave breaking statistics on wind stress and wave development. Presented at the IUTAM Breaking Waves Symposium, Sydney, Australia, 15-19 July 1991.

Kawai, S. 1979 Generation of initial wavelets by instability of a coupled shear flow and their evolution to wind waves. J. Fluid Mech. 93, 661-703.

Kawai, S., K. Okuda and Y. Toba 1977 Field data support of three-seconds power law and $g^{*} \sigma^{-4}$ spectral form for growing wind waves. J. Oceanogr. Soc. Japan 33, 137-150.

Kennedy, R.M. 1992 Sea surface dipole sound source dependence on wave-breaking variables. J. Acoust. Soc. Am. 91, 1974-1982.

Kerman, B.R. 1984 Underwater sound generation by breaking wind waves. J. Acoust. Soc. Am. 75, 149-165. 
Kerman, B.R. 1988 Sea Surface Sound: Natural Mechanisms of Surface Generated Noise in the Ocean. Kluwer Academic, Dordrecht, Netherlands.

Kerman, B.R. 1992 Natural Physical Sources of Underwater Sound:Sea Surface Sound (2). Kluwer Academic, Dordrecht, Netherlands.

Keulegan, J.H. 1951 Wind tides in small closed channels. J. Res. Natl. Bur. Stand. 46, 358381.

Kitaigorodskii, S.A. 1962 Applications of the theory of similarity to the analysis of windgenerated wave motion as a stochastic process. Bull. Acad. Sci. USSR Geophys. 1, 73.

Knudsen, V.O., R.S. Alford and J.W. Emling 1948 Underwater ambient noise. J. Mar. Res. 7, 410-429.

Kolaini, A., R.A. Roy and L.A. Crum 1991 An investigation of the acoustic emissions from a bubble plume. J. Acoust. Soc. Am. 89, 2452-2455.

Komen, G.J., S. Hasselmann and K. Hasselmann 1984 On the existence of a fully developed wind-sea spectrum. J. Phys. Oceanogr. 13, 816-827.

Kuo, E.Y.T. 1968 Deep-sea noise due to surface motion. J. Acoust. Soc. Am. 43, 1017-1024.

Lamarre, E. and W.K. Melville 1991 Air entrainment and dissipation in breaking waves. Nature 351, 469-472. 


\section{References}

Lamarre, E. and W.K. Melville 1992 Void-fraction measurements and sound-speed fields in bubble plumes generated by breaking waves. Submitted to J. Acoust. Soc. Am.

Large, W.G. and S. Pond 1981 Open ocean momentum flux measurements in moderate to strong winds. J. Phys. Oceanogr. 11, 324-336.

Larson, T.R. and J.W. Wright 1975 Wind-generated gravity-capillary waves: Laboratory measurements of temporal growth rates using microwave backscatter. J. Fluid. Mech. 70, $417-436$.

Lemon, D.D., D.M. Farmer and D.R. Watts 1984 Acoustic measurements of wind speed and precipitation over a continental shelf. J. Geophys. Res. 89, 3462-3472.

Loewen, M.R. 1991 Laboratory Measurements of the Sound Generated by Breaking Waves. PhD Thesis, Massachusetts Institute of Technology p. 208.

Loewen, M.R. and W.K. Melville 1991a Microwave backscatter and acoustic radiation from breaking waves. J. Fluid Mech. 224, 601-623.

Loewen, M.R. and W.K. Melville 1991b A model of the sound generated by breaking waves. J. Acoust. Soc. Am. 90, 2075-2080.

Loewen, M.R. and W.K. Melville 1992 An experimental investigation of the collective oscillations of bubble plumes entrained by breaking waves. Submitted to J. Acoust. Soc. Am. 
Longuet-Higgins, M.S. 1951 A theory of the origin of micro-seisms. Phil. Trans. R. Soc. A 243, 1-35.

Longuet-Higgins, M.S. 1976 On the nonlinear transfer of energy in the peak of a gravity wave spectrum: a simplified model. Proc. R. Soc. London A 347, 311-328.

Longuet-Higgins, M.S. and N.D. Smith 1983. Measurements of breaking waves by a surface jump meter. J. Geophys. Res. 88, 9823-9831.

Lu, N.Q., A. Prosperetti and S.W. Yoon 1990 Underwater noise emissions from bubble clouds. IEEE J. Ocean. Engg. 15, 275-281.

Maat, N., C. Kraan and W.A. Oost 1991 The roughness of wind waves. Boundary-Layer Met. 54, 89-103.

Marsh, H.W. 1963 Origin of the Knudsen spectra. J. Acoust. Soc. Am. 35, 409-410.

Medwin, H. and M.M. Beaky 1989 Bubble sources in the Knudsen sea noise spectra. J. Acoust. Soc. Am. 86, 1124-1130.

Medwin, H. and A.C. Daniel Jr. 1990 Acoustical measurements of bubble production by spilling breakers. J. Acoust. Soc. Am. 88, 408-412.

Melville, W.K. 1992 Energy dissipation by breaking waves. J. Phys. Oceanogr. Sub judice. 
Melville, W.K. and R.J. Rapp 1985 Momentum flux in breaking waves. Nature 317, 514516.

Melville, W.K., M.R. Loewen, F.C. Felizardo, A.T. Jessup and M.J. Buckingham 1988 Acoustic and microwave signatures of breaking waves. Nature 336, 54-59.

Miles, J.W. 1957 On the generation of surface waves by shear flows. J. Fluid Mech. 3, 185204.

Minnaert, M. 1933 On musical air bubbles and the sound of running water. Phil. Mag. $16,235-248$.

Oakey, N.S. and J.A. Elliot 1982 Dissipation within the surface mixed layer. J. Phys. Oceanogr. 12, 171-185.

Oakey, O.H. and J.B. Lozow 1977 The Resolution of Directional Wave Spectra Using the Maximum Likelihood Method, MIT Dept. of Ocean Engg Tech Report No. 77-1.

Penhallow, W.S. and F.T. Dietz 1964 Correlation of 630-cps shallow-water ambient noise with wind speed and waveheight. J. Acoust. Soc. Am. 36, 2149-2151.

Perrone, A.J. 1969 Deep-ocean ambient noise spectra in the northwest Atlantic. J. Acoust. Soc. Am. 46, 762-770.

Phillips, O.M. 1958 The equilibrium range in the spectrum of wind-generated waves. J. Fluid Mech. 4, 426-434. 
Phillips, O.M. 1985 Spectral and statistical properties of the equilibrium range in windgenerated gravity waves. J. Fluid Mech. 156, 505-531.

Piggott, C.L. 1964 Ambient sea noise at low frequencies in shallow waters off the Scotian Shelf. J. Acoust. Soc. Am. 36, 2152-2163.

Plant, W.J. 1982 A relationship between wind stress and wave slope. J. Geophys. Res. 87, 1961-1967.

Plant, W.J. and J.W. Wright 1977 Growth and equilibrium of short gravity waves in a wind-wave tank. Fluid Mech. 82, 767-793.

Press, W.H., B.P. Flannery, S.A. Teukolsky and W.T. Vetterling 1986 Numerical Recipes: The Art of Scientific Computing. Cambridge University Press, Cambridge, UK.

Prosperetti, A. 1988 Bubble-related ambient noise in the ocean. J. Acoust. Soc. Am. 84, 1042-1054.

Pumphrey, H.C. and J.E. Ffowcs Williams 1990 Bubbles as source of ambient noise. IEEE J. Oceanic Engg. 15, 268-274.

Rapp, R.J. and W.K. Melville 1990 Laboratory measurements of deep-water breaking waves. Phil. Trans. Roy. Soc. Lond. A 331, 735-800.

Rudnick, P. 1967 Motion of a large spar bouy in sea waves. J. Ship Res. 257-267. 
Sell, W. and K. Hasselmann 1972 Computation of nonlinear energy transfer fro JONSWAP and empirical wind wave spectra. Rep. Inst. Geophys., Univ. of Hamburg.

Shaw, P.T., D.R. Watts and H.T. Rossby 1978 On the estimation of oceanic wind speed and stress from ambient noise measurements. Deep-Sea Res. 25, 1225-1233.

Shemdin, O.H. and E.Y. Hsu 1967 Direct measurement of aerodynamic pressure above a simple progressive gravity wave. J. Fluid Mech. 30, 403-416.

Shooter, J.A. and M.L. Gentry 1981 Wind generated noise in the Parece Vela Basin. J. Acoust. Soc. Am. 70, 1757-1761.

Snyder, R.L., F.W. Dobson, J.A. Elliot and R.B. Long 1981 Array measurements of atmospheric pressure fluctuations above surface gravity waves. J. Fluid Mech. 102, 1-59.

Soloviev, A.V., N.V. Vershinsky and V.A. Bezverchnii 1988 Deep sea res. 35, 1859-1874.

Stewart, R.H. and C. Teague 1980 Dekameter radar observations of ocean wave growth and decay. J. Phys. Oceanogr. 10, 128-143.

SWAMP 1985 Ocean Wave Modelling. Plenum Press, New York, NY.

Tennekes, H. and J.L. Lumley 1972 A First Course in Turbulence. MIT Press, Cambrdige, MA.

Thorpe, S.A. 1992 Energy loss by breaking waves. J. Phys. Oceanogr. Sub judice. 
References

Thorpe, S.A. 1992a Bubble clouds and the dynamics of the upper ocean. Q. J. R.

Meteorol. Soc. 118, 1-22.

Thorpe, S.A. and P.N. Humphries 1980 Bubbles and breaking waves. Nature 283, 463465.

Toba, Y. 1973 Local balance in the air-sea boundary processes. III. On the spectrum of wind waves. J. Oceanogr. Soc. Japan 29, 209-220.

Toba, Y. and M. Koga 1986 A parameter describing overall conditions of wave-breaking, whitecapping, sea-spray production and wind stress. In Oceanic Whitecaps and Their Role in Air-Sea Exchange Processes, E.C. Monahan and G. MacNiocaill, eds. Reidel, Boston, MA, 37-47.

Urick, Robert J. 1986 Ambient Noise in the Sea. Peninsula Publishing, Los Altos, CA.

Urick, Robert J. 1975 Principles of Underwater Sound. McGraw-Hill Book Company, NY.

Vagle, S., W.G. Large and D.M. Farmer 1990 An evaluation of the WOTAN technique of inferring oceanic winds from underwater ambient sound. J. Atmos. Ocean. Tech. 7, 576595.

WAMDIG 1988 The WAM model - a third generation ocean wave prediction model. J. Phys. Oceanogr. 18, 1775-1810.

Weissman, M.A., S.S. Ataktürk and K.B. Katsaros 1984 Detection of breaking events in a wind-generated wave field. J. Phys. Oceanogr. 14, 1608-1619. 
Weller, R.A., M.A. Donelan, M.G. Briscoe and N.E. Huang 1991 Riding the crest: a tale of two wave experiments. Bull. Am. Meterol. Soc. 72, 163-183.

Wenz, G.M. 1962 Acoustic ambient noise in the ocean: spectra and sources. J. Acoust. Soc. Am. 34, 1936-1956.

Wilson, J.H. 1983 Wind-generated noise modeling. J. Acoust. Soc. Am. 73, 211-216.

Wu, H.Y., E.Y. Hsu and R.L. Street 1979 Experimental study of nonlinear wave-wave interaction and white-cap dissipation of wind-generated waves. Dyn. Atmos. Oceans 3, 55-78.

Yoon, S.W., L.A. Crum, A. Prosperetti and N.Q. Lu 1991. An investigation of the collective oscillations of a bubble cloud. J. Acoust. Soc. Am. 89, 700-706. 


\section{APPENDIX}

\section{A.1 Directional hydrophone characteristics.}

\section{A.1.1 The hydrophone equation.}

In this section, we discuss the method used to calculate the noise spectrum level using the directional hydrophone we used in NOBS. The ambient noise level N(f) [dB re $\left.\mu \mathrm{Pa}^{2} / \mathrm{Hz}\right]$ for an equivalent omnidirectional hydrophone can be computed from the raw power spectrum $R(f)$

$$
N(f)=R(f)-S A+S L(f)+A G(f)
$$

where $\mathrm{R}(\mathrm{f})$ is in $\mathrm{dB}$ re $1 \mathrm{Volt}{ }^{2} / 1 \mathrm{~Hz}, \mathrm{SA}$ is the signal amplification, and SL is the sensitivity level of the hydrophone in $\mathrm{dB}$ re $1 \mu \mathrm{Pa}^{2} / 1 \mathrm{Volt}^{2}$. SA was set at $50 \mathrm{~dB}$ throughout the NOBS observation period. The array gain AG due to spatial filtering by the directional hydrophone is defined as (Dyer, 1989)

$$
A G \equiv 10 \log \frac{\int_{\Omega} \mathrm{S}(\Omega) \mathrm{d} \Omega}{\int_{\Omega} \mathrm{S}(\Omega) \mathrm{B}^{2}(\Omega) \mathrm{d} \Omega}
$$

where $\Omega$ is the solid angle, $B(\Omega)$ is the beam pattern of the hydrophone and $S(\Omega)$ is the sound source pattern. 


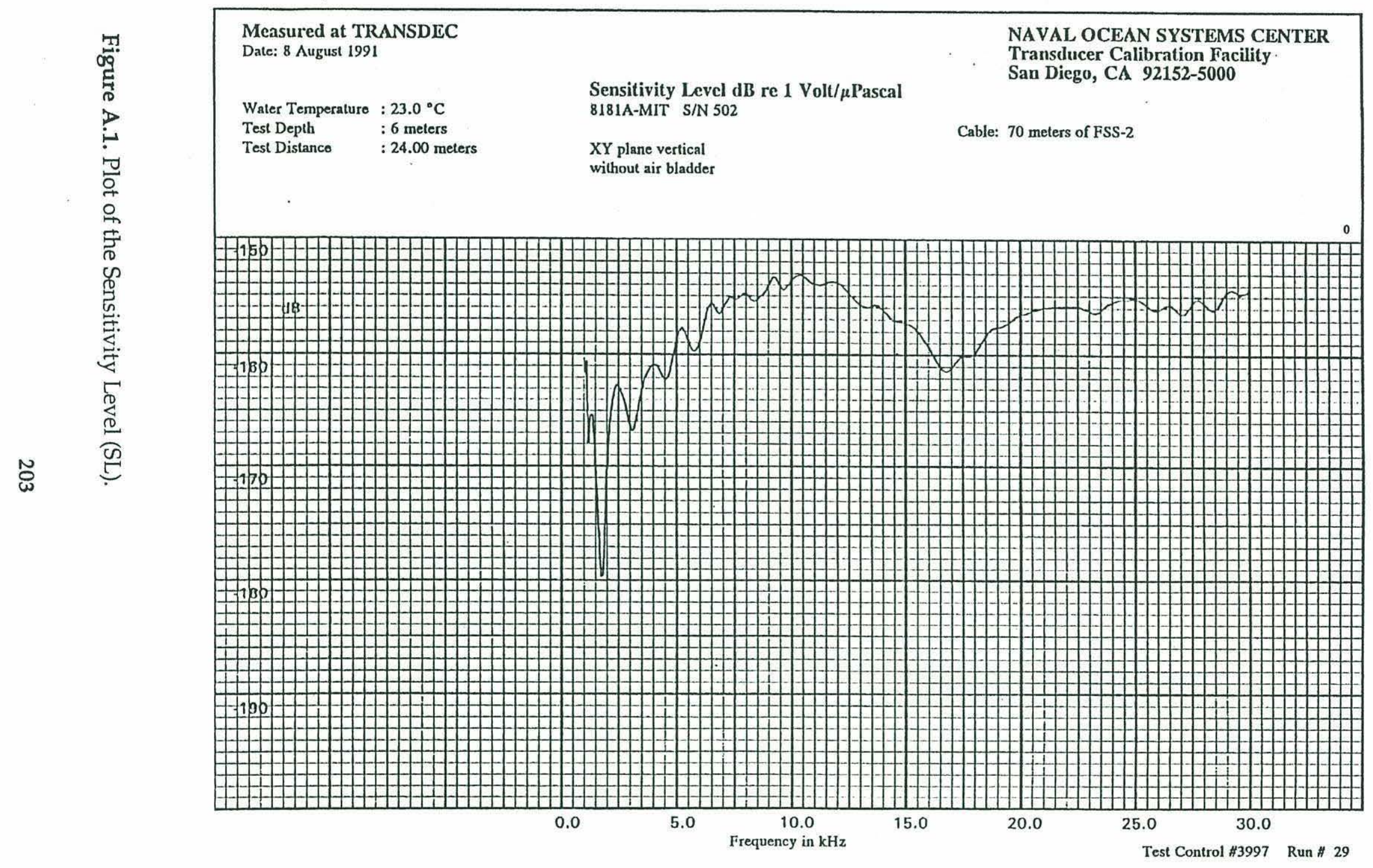




\section{Appendix}

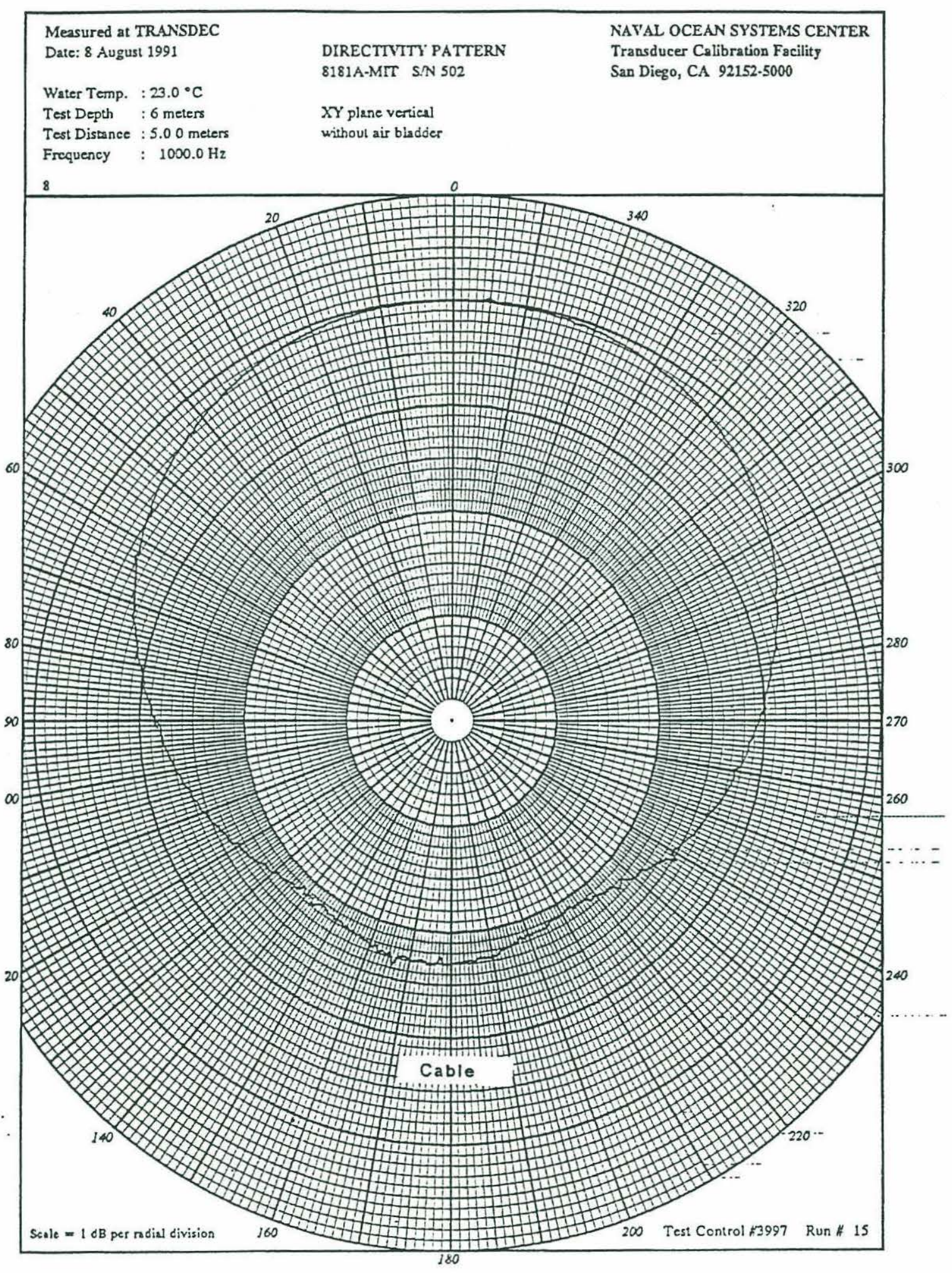

Figure A.2. a) Plot of the beam pattern of the hydrophone at $1 \mathrm{kHz}$. 


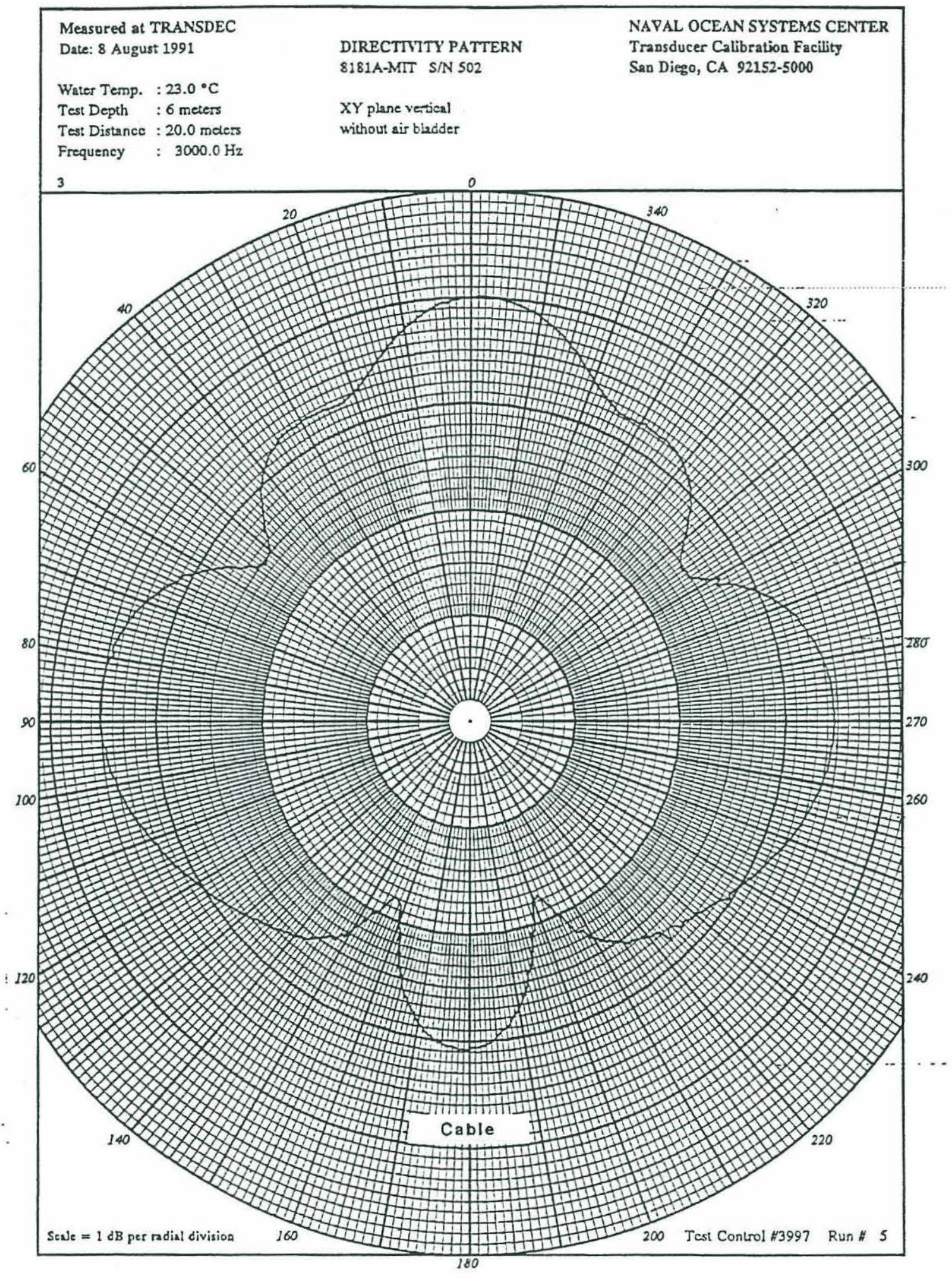

Figure A.2. b) Plot of the beam pattern of the hydrophone at $3 \mathrm{kHz}$. 


\section{Appendix}

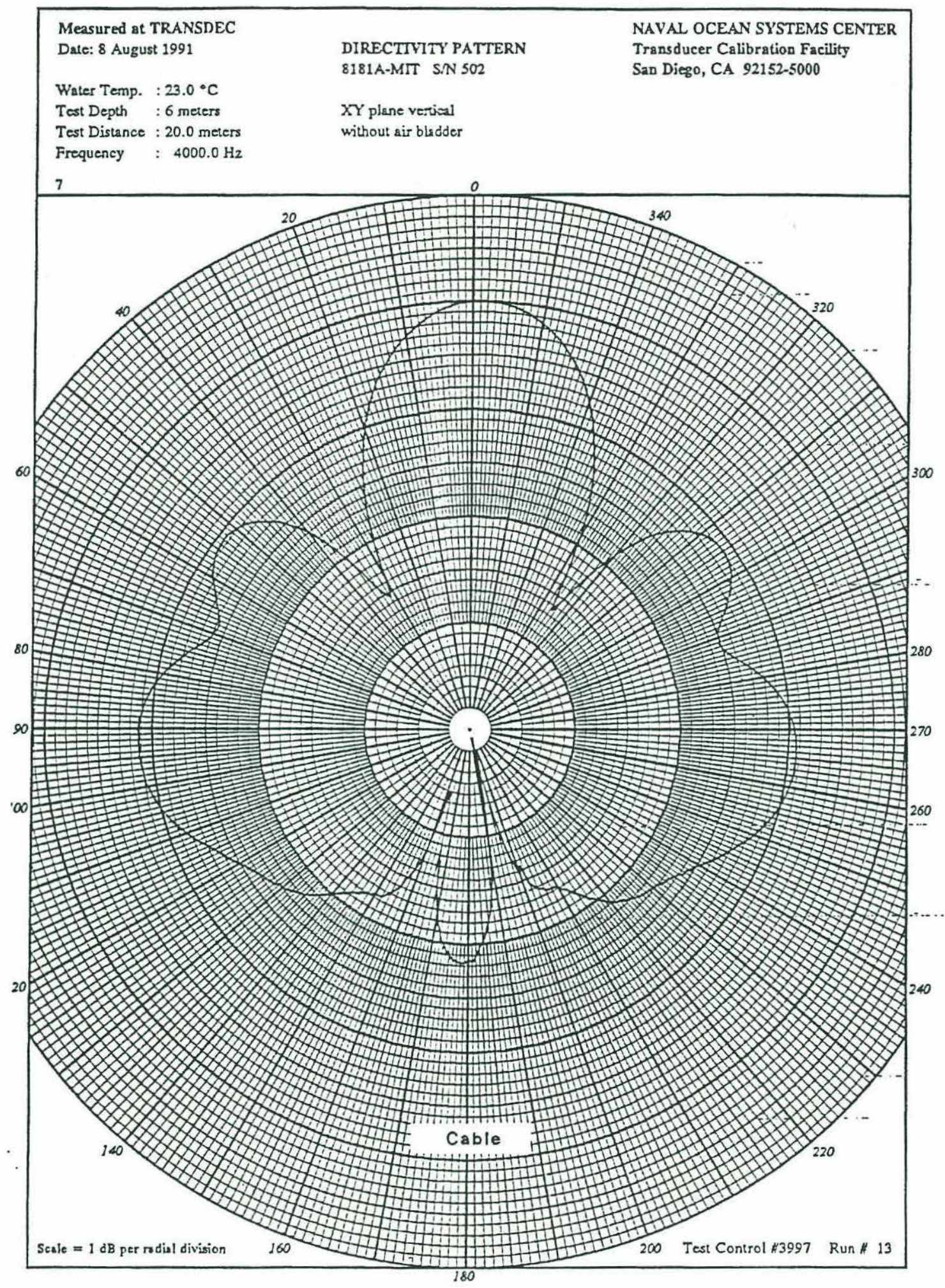

Figure A.2. c) Plot of the beam pattern of the hydrophone at $4 \mathrm{kHz}$. 


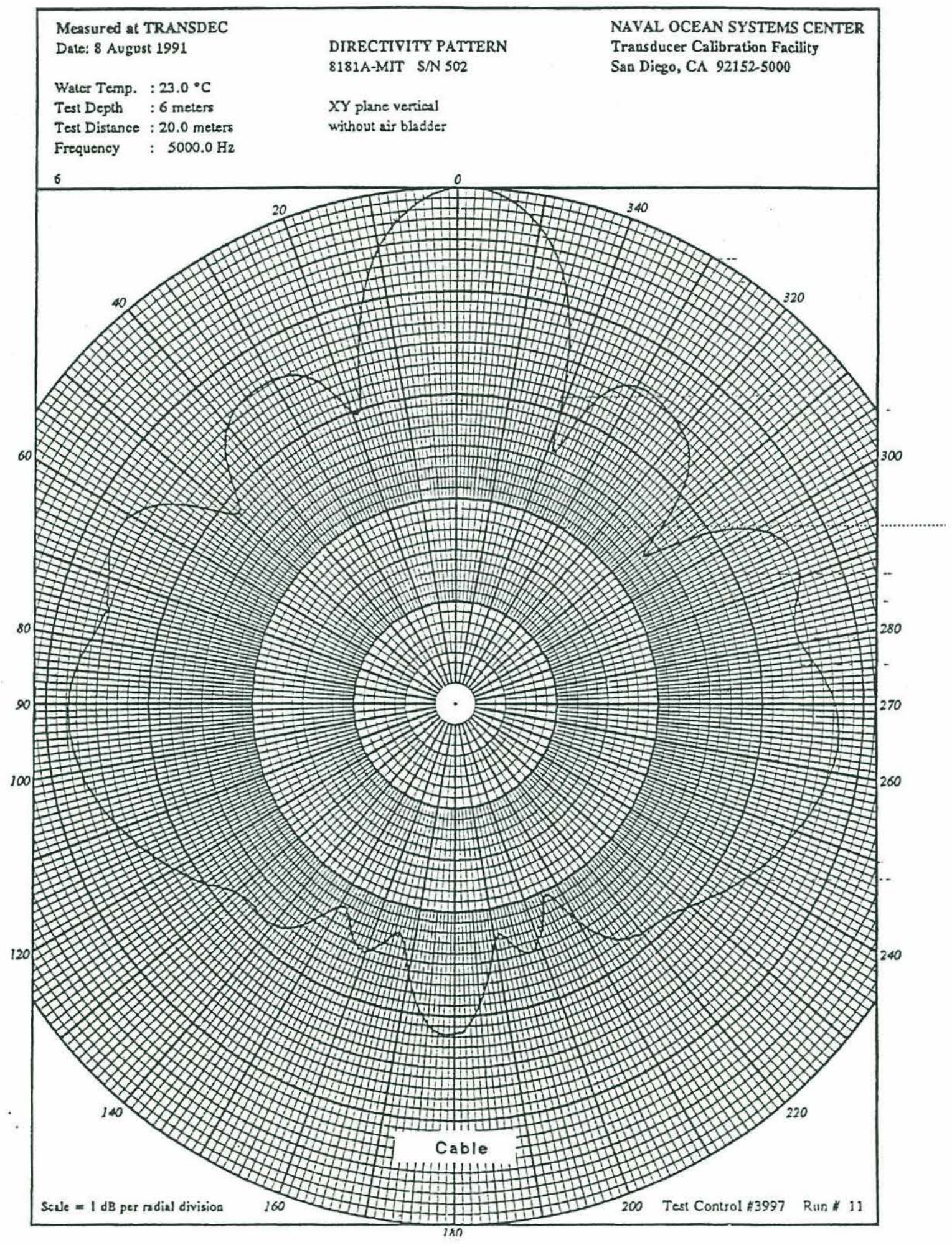

Figure A.2. d) Plot of the beam pattern of the hydrophone at $5 \mathrm{kHz}$. 


\section{Appendix}

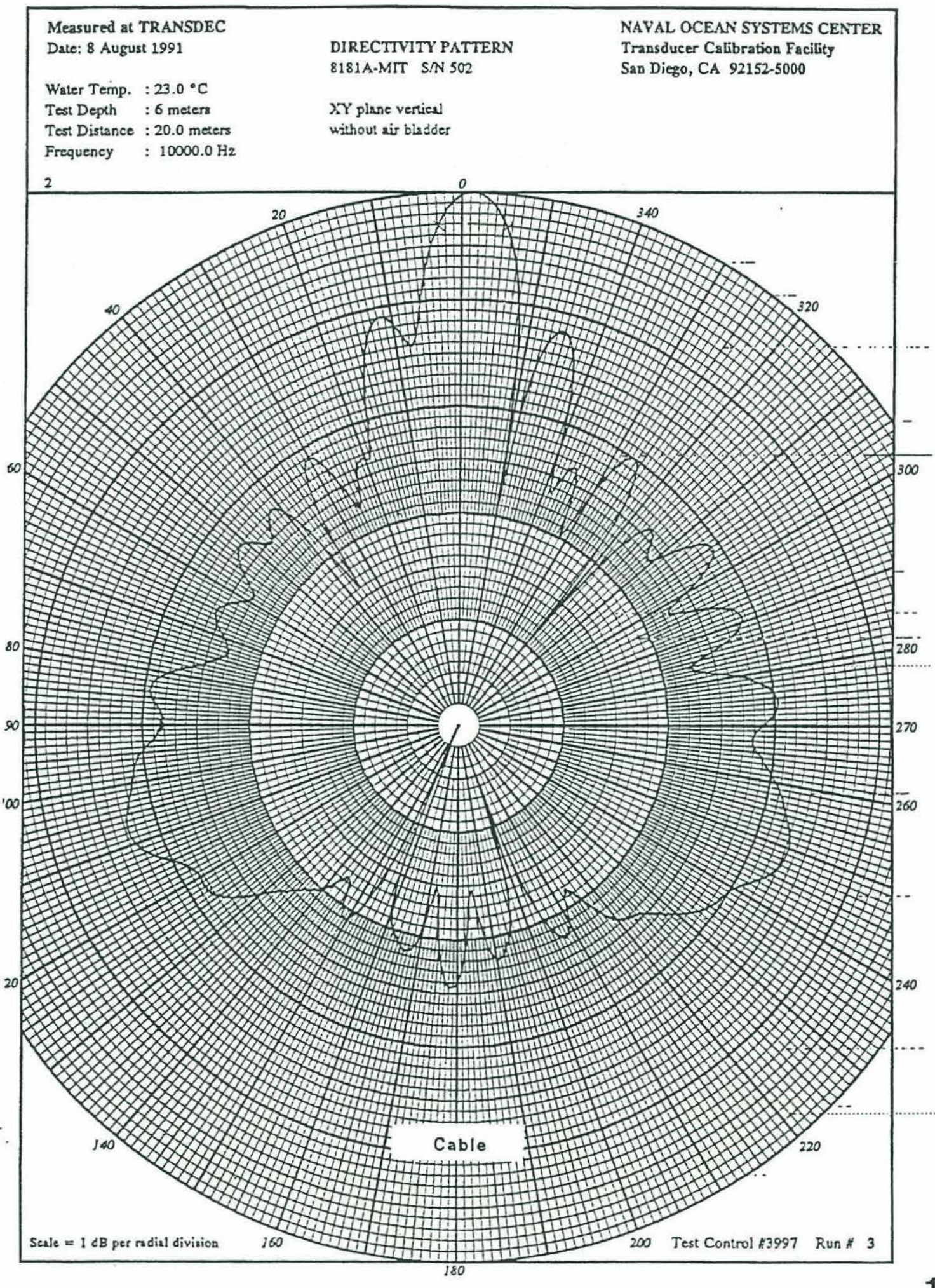

Figure A.2. e) Plot of the beam pattern of the hydrophone at $10 \mathrm{kHz}$. 


\section{Appendix}

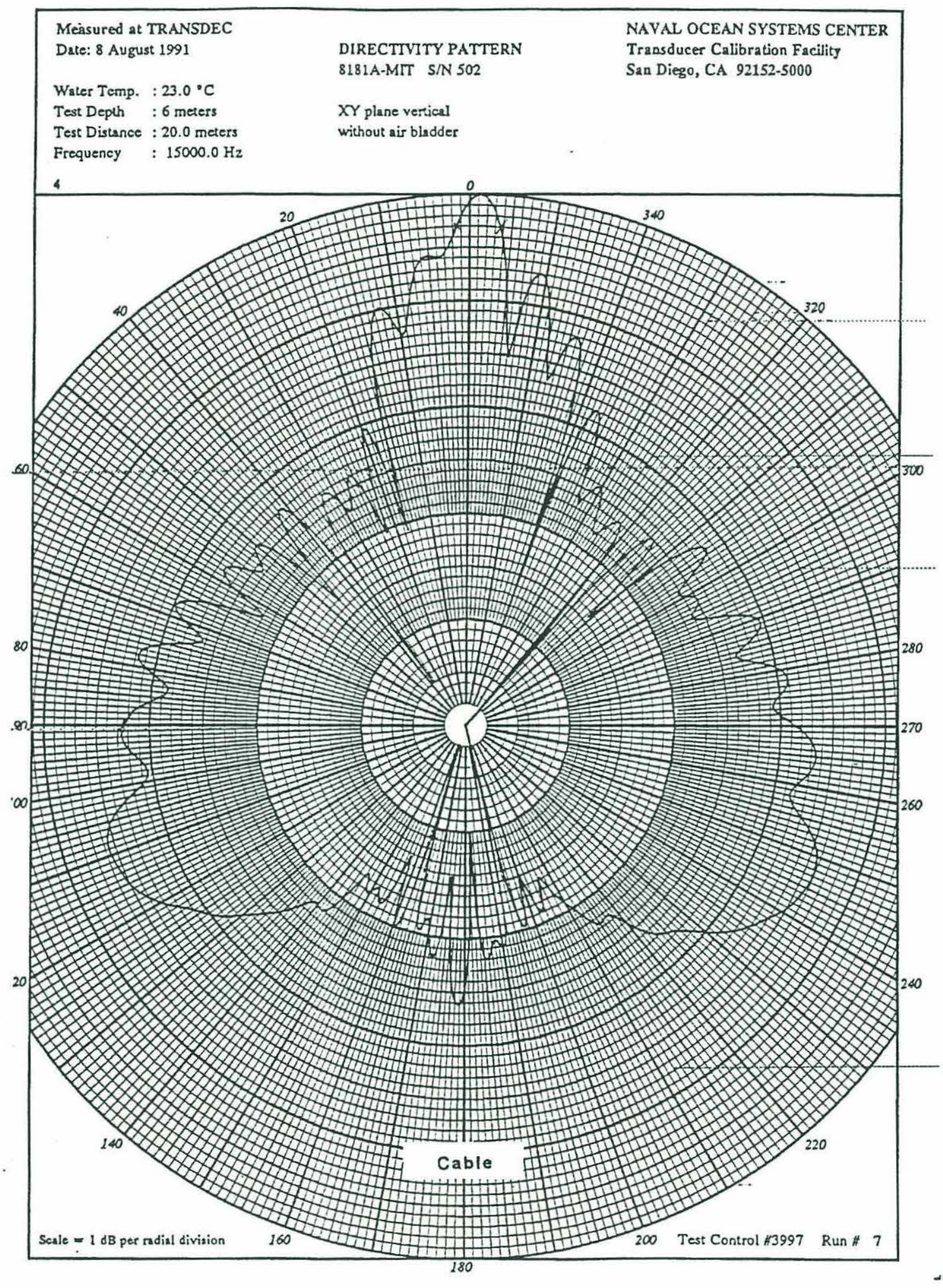

Figure A.2. f) Plot of the beam pattern of the hydrophone at $15 \mathrm{kHz}$. 


\section{Appendix}

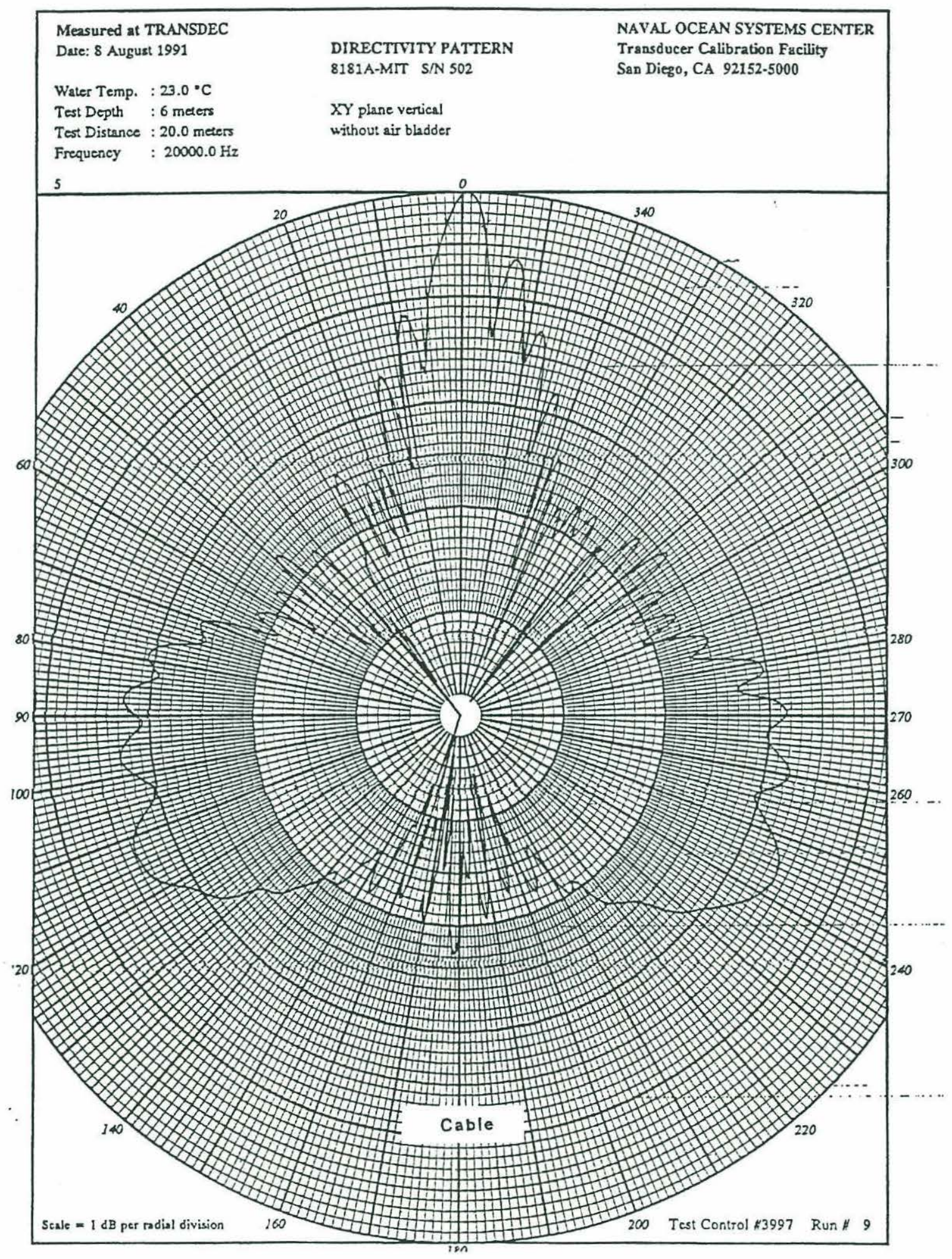

Figure A.2. g) Plot of the beam pattern of the hydrophone at $20 \mathrm{kHz}$. 


\section{A.1.2 Calibration results.}

In this experiment we used an ITC-8181A positioned at the focus of a 42 " foam filled parabolic dish. The hydrophone was then calibrated at the Naval Ocean Systems Center (NOSC) in San Diego, California. The diagrams from the calibration report are shown in Figures A.1 and A.2. Figure A.1 shows the plot of the Sensitivity Level (SL) while Figure A.2 shows the beam pattern $B^{2}\left(\phi_{h}\right)$ of the hydrophone at 1, 3, 4, 5, 10, 15 and $20 \mathrm{kHz}$ where $\phi_{\mathrm{h}}$ is the angle from the axis of the hydrophone. Radial symmetry with respect to the hydrophone axis was assumed.

\section{A.1.3 Array gain computations.}

In addition to the beam pattern $B^{2}(\Omega)$, we also need the sound source pattern $S(\Omega)$ to evaluate AG(f) (Equation 3.2). To estimate the ambient noise source pattern, we followed the formalism of Urick (1986, p.5-1ff.) which is reviewed here. He proposed that the sea surface can be modeled as a distribution of random and densely packed sound sources each radiating sound with intensity (Figure A.3)

$$
I(\phi)=I_{0} \cos ^{\mathrm{n}} \phi
$$

where $\phi$ is the angle from the receiver at depth $h$ and horizontal distance $r$ to the source. For a ring of sources of area $\mathrm{d} A$, the intensity is

$$
\mathrm{d} I=\frac{I(\phi)}{h^{2}+r^{2}} \mathrm{~d} A=\frac{I(\phi)}{l^{2}} 2 \pi r \mathrm{~d} r .
$$




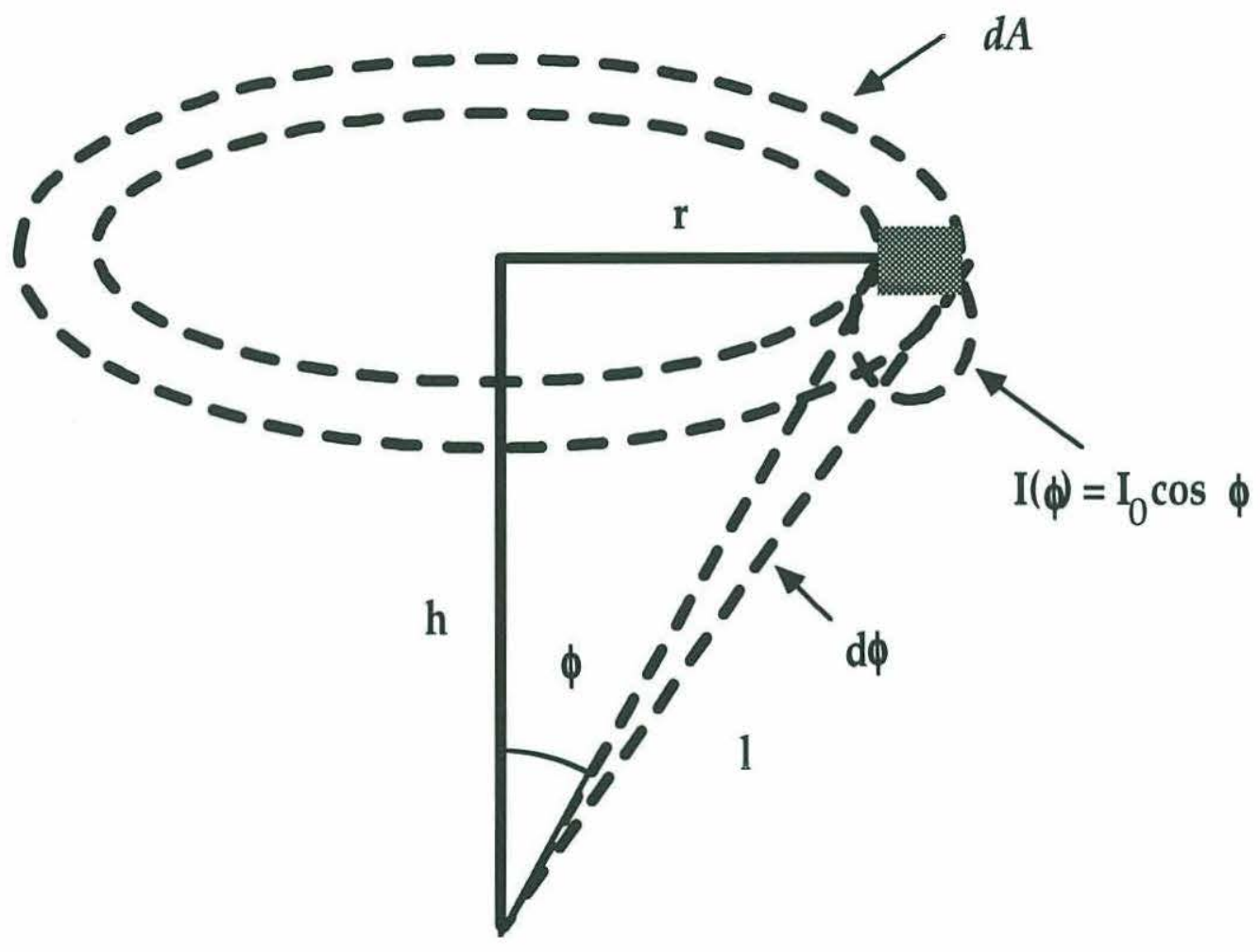

Figure A.3. Geometry of Urick's (1986) model for the distribution of sound sources at the ocean surface. 

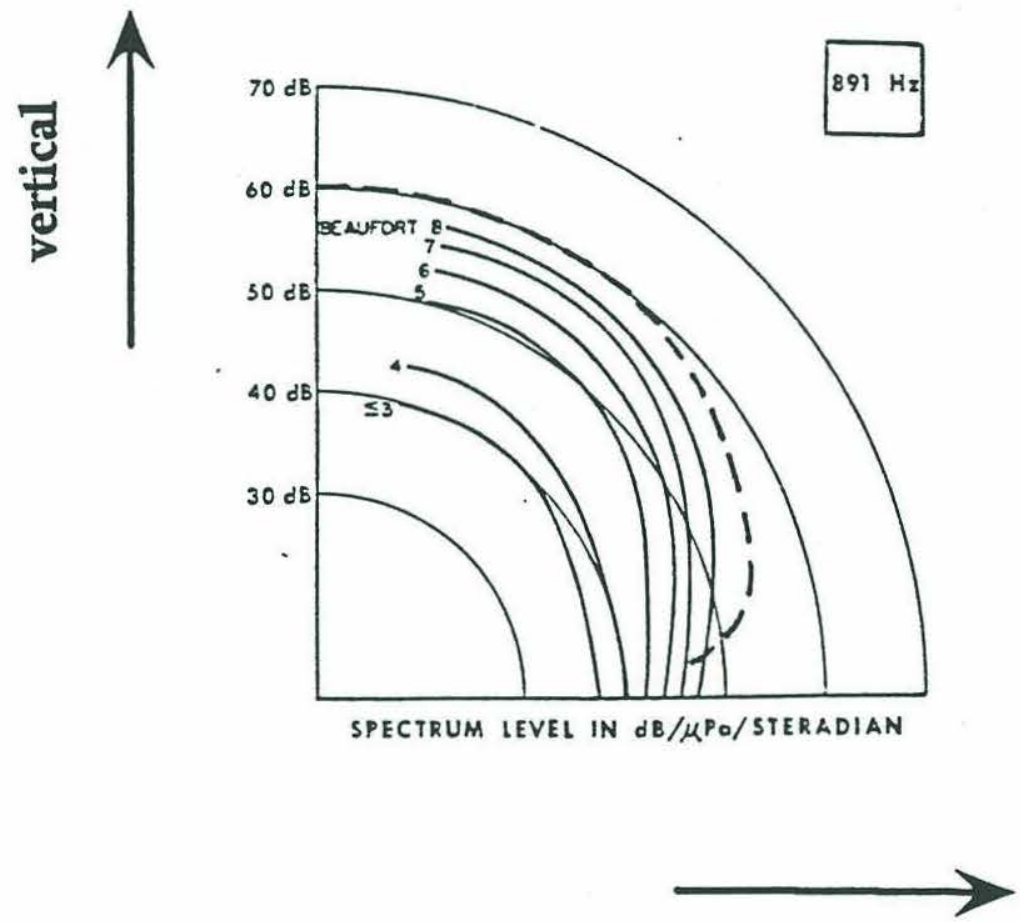

horizontal

. Figure A.4. Urick's (1986) $\cos \phi$ model of the vertical distribution of ambient noise (dashed line) agrees well with measurements by Axelrod et al (1965) at $891 \mathrm{~Hz}$ (adapted from Urick, 1986). 
From Figure A.3, it is easy to show that

$$
\begin{aligned}
& r=h \tan \phi, \\
& l=h \sec \phi, \\
& \mathrm{d} r=h \sec ^{2} \phi \mathrm{d} \phi .
\end{aligned}
$$

Hence

$$
\mathrm{d} I=2 \pi I_{0} \cos ^{\mathrm{n}} \phi \tan \phi \mathrm{d} \phi
$$

The intensity per unit solid angle $\Omega$ is then

$$
\mathrm{S}(\Omega)=\frac{\mathrm{d} I}{\mathrm{~d} \Omega}=\frac{2 \pi I_{0} \cos ^{\mathrm{n}} \phi \tan \phi \mathrm{d} \phi}{2 \pi \sin \phi \mathrm{d} \phi}=I_{0} \cos ^{\mathrm{n}-1} \phi
$$

For a dipole, $\mathrm{n}=2$ and

$$
\mathrm{S}(\Omega)=\frac{\mathrm{d} I}{\mathrm{~d} \Omega}=I_{0} \cos \phi
$$

Figure A.4 shows a plot taken from Urick (1986, p. 5-10) comparing Equation A.6 with the ambient noise directional pattern obtained by Axelrod, Schoomer \& Von Winkle (1965). The figure shows good agreement between their data and Equation A.6.

Integrating the denominator of Equation 3.2 is complicated by the different orientations of the coordinate axes of $\mathrm{S}$ and $\mathrm{B}^{2}$. While $\mathrm{S}$ is radially symmetric about the z-axis, the axis of radial symmetry of the hydrophone during NOBS was pointed 
at an angle $45^{\circ}$ from the $\mathrm{z}$-axis. Hence need to derive a function that will transform data from the sound source coordinate system $(r, \phi, \theta)$ to the hydrophone coordinate system $\left(r, \phi_{h}, \theta_{h}\right)$.

In Figure A.5, the position vector for a point $A$ on the hydrophone axis in the sound source coordinate system is

$$
\boldsymbol{A}=A\left[\begin{array}{c}
0 \\
\cos 45^{\circ} \\
\cos 45^{\circ}
\end{array}\right]=A\left[\begin{array}{c}
0 \\
\frac{1}{\sqrt{2}} \\
\frac{1}{\sqrt{2}}
\end{array}\right] \text {. }
$$

The position vector for a point $B$ in the sound source coordinate system is

$$
\boldsymbol{B}=B\left[\begin{array}{c}
\sin \phi \cos \theta \\
\sin \phi \sin \theta \\
\cos \phi
\end{array}\right] .
$$

The coordinate transformation for the angles can be obtained from the dot product of $A$ and $B$

$$
\begin{gathered}
\boldsymbol{A} \cdot \boldsymbol{B}=\frac{A B}{\sqrt{2}}(\sin \phi \sin \theta+\cos \phi)=\frac{A B}{\sqrt{2}} \cos \phi_{\mathrm{h}} \\
\phi_{\mathrm{h}}=\cos ^{-1}(\sin \phi \sin \theta+\cos \phi) .
\end{gathered}
$$

Hence, the denominator of the equation for AG in terms of the coordinates of the hydrophone axis is 


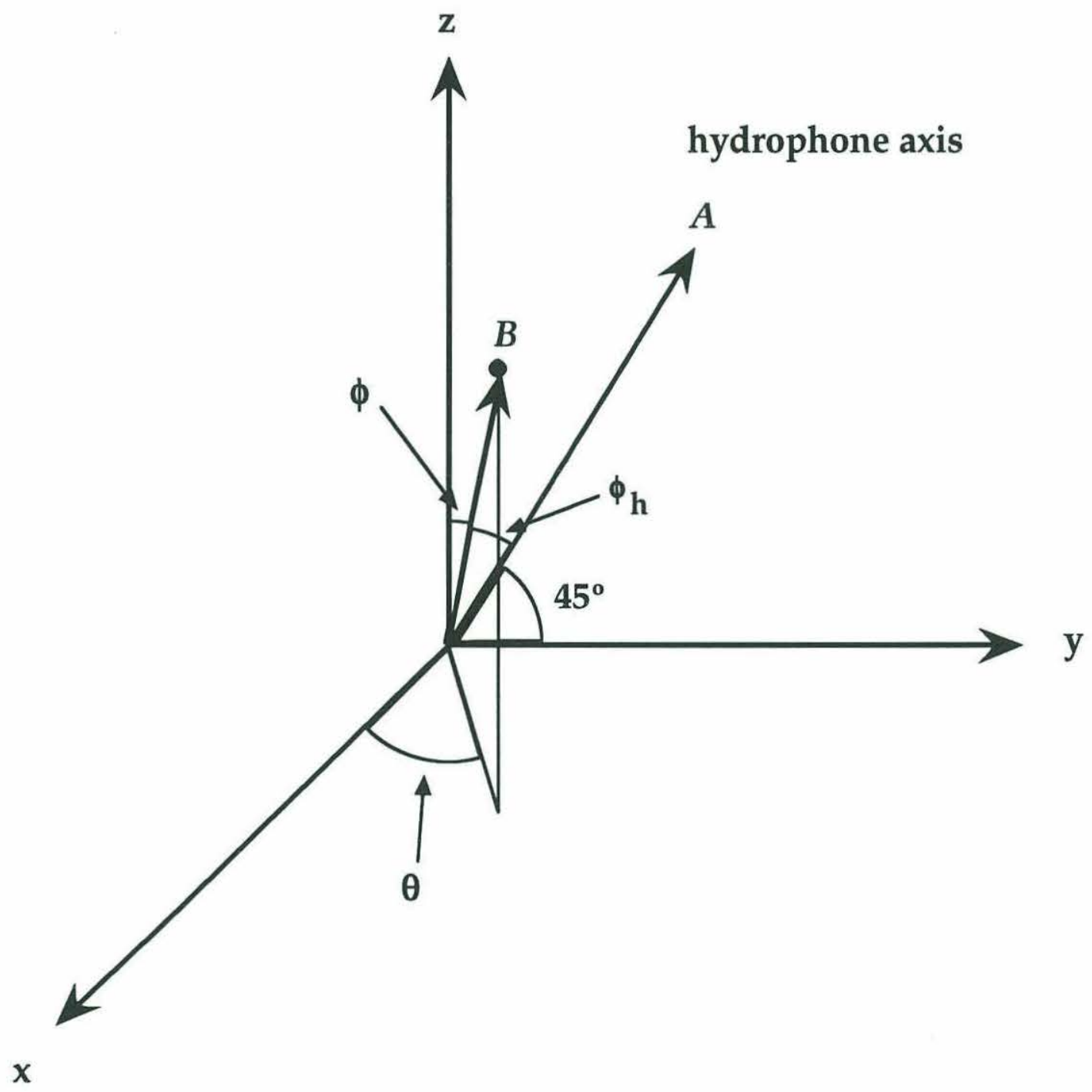

Figure A.5. Geometry for the coordinate transformation from the sound source coordinate system to the hydrophone coordinate system. 


$$
\int_{\Omega} \mathrm{S}(\Omega) \mathrm{B}^{2}(\Omega) \mathrm{d} \Omega=2 \pi \int_{0}^{\frac{\pi}{2}} \mathrm{~S}\left(\phi_{\mathrm{h}}\right) \mathrm{B}^{2}\left(\phi_{\mathrm{h}}\right) \mathrm{d} \phi_{\mathrm{h}}
$$

which can be integrated numerically. The numerator of AG can be integrated analytically in the sound source coordinate system

$$
\int_{\Omega} \mathrm{S}(\Omega) \mathrm{d} \Omega=2 \pi I_{0} \int_{0}^{\frac{\pi}{2}} \cos \phi \mathrm{d} \phi=2 \pi I_{0} .
$$

Table A.1. Numerical values of AG and SL used in Equation 3.1.

\begin{tabular}{|c|c|c|}
\hline $\mathrm{f}(\mathrm{kHz})$ & $\mathrm{AG}$ & $\mathrm{SL}$ \\
\hline 2 & 4.3 & -162 \\
\hline 3 & 8.7 & -162 \\
\hline 4 & 11.2 & -161 \\
\hline 5 & 13.6 & -158 \\
\hline 6 & 15.8 & -157 \\
\hline 7 & 17.6 & -156 \\
\hline 8 & 19.1 & -155 \\
\hline 9 & 20.3 & -153 \\
\hline 10 & 21.1 & -153 \\
\hline 11 & 21.6 & -153 \\
\hline 12 & 21.8 & -153 \\
\hline 13 & 21.8 & -154 \\
\hline 14 & 21.7 & -155 \\
\hline 15 & 21.6 & -156 \\
\hline
\end{tabular}


Appendix

Figure A. 6 shows a plot of AG(f). The values of AG computed from the available beam patterns are indicated by hollow circles (0). We also computed the value of AG based on an omnidirectional sound source assumption

$$
\mathrm{S}(\Omega)=1
$$

These are shown as filled circles $(\bullet)$ in the figure. Figure A.6 suggests that, for our hydrophone, the value of AG is not sensitive to the differences the assumed source level distribution. Figure A.6 also shows the curve we used to interpolate the data for the frequency range we examined. The numerical values of the curve are given in Table A.1. 


\section{Appendix}

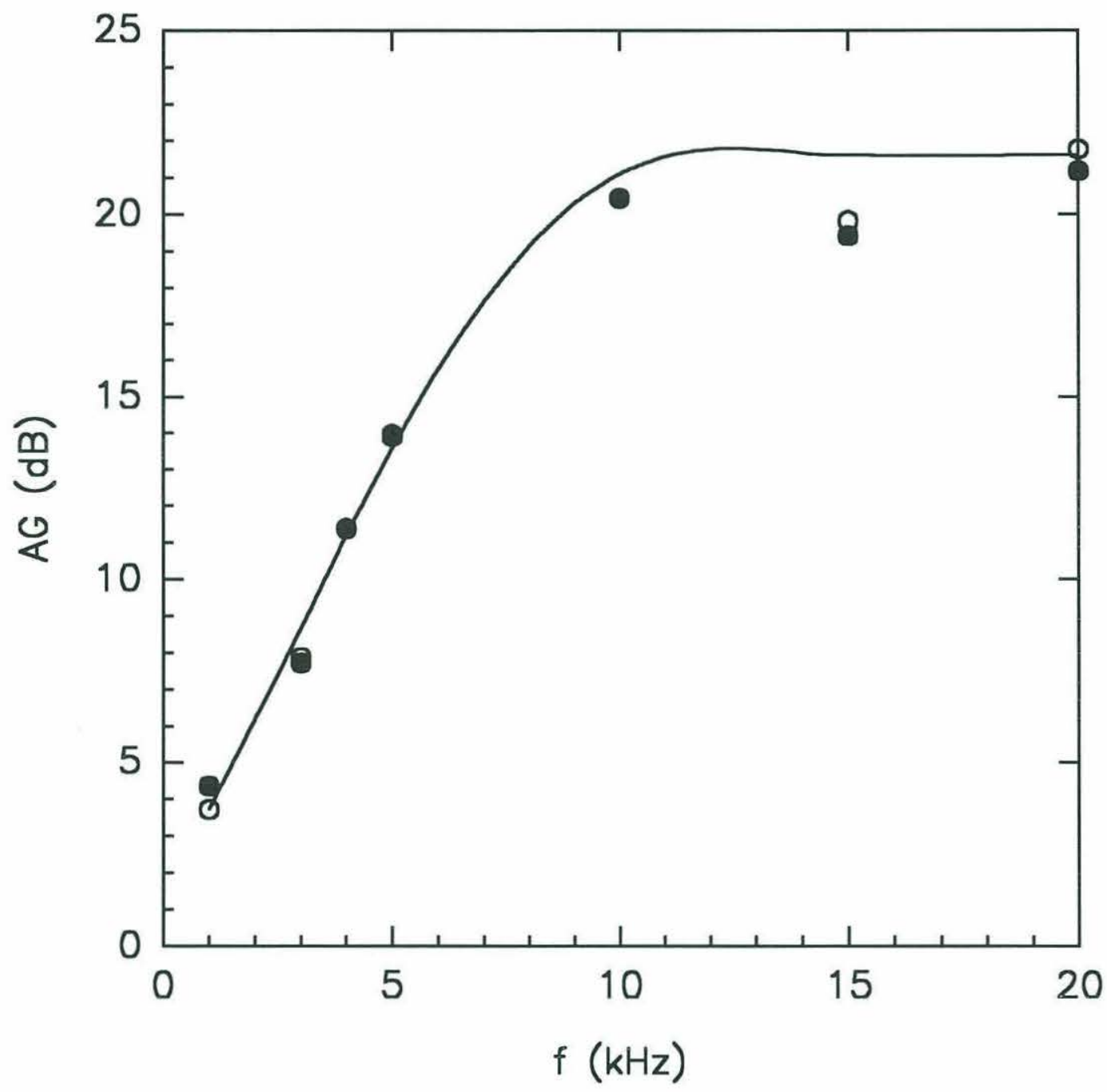

Figure A.6. Plot showing the array gain (AG) of the directional hydrophone computed from the available beam patterns. Dipole source assumed - 0 , Omnidirectional source assumed - $\bullet$. The line indicates the fit to the data used in computing N(f) [Equation 3.1]. 